\title{
Uma Abordagem Bayesiana para Modelos Auto-Regressivos Periódicos - PAR
}

\author{
Claudia Fernanda Freitas Hutter
}

Orientador: Prof. Dr. Marinho Gomes de Andrade Filho

Dissertação apresentada ao Instituto de Ciências Matemáticas e de Computação

- USP, como parte dos requisitos para obtenção do título de Mestre em Ciências - Área de Ciências de Computação e Matemática Computacional 
Dedico este trabalho aos meus pais Fred e Beth e ao Carlos. 


\section{Agradecimentos}

A realização deste trabalho não seria possivel sem o apoio de algumas pessoas e instituições que, de alguma forma, contribuíram para esta dissertação e as quais expresso meus sinceros agradecimentos.

Ao Prof. Dr. Marinho Gomes de Andrade, pela orientação segura, pelo incentivo, pela amizade e por tudo que me ensinou no decorrer deste trabalho.

Aos Profs. Dr. Jorge Alberto Achar e Josemar Rodrigues, pelos valiosos comentários e sugestões na participação do exame de qualificação.

À todos meus amigos e especialmente, Ana Cláudia, Gilberto, Silvana, Patrícia, Josmar, José Roberto e Josenildo pelo apoio e principalmente pela amizade.

Aos meus pais Fred e Beth pelo carinho, pela compreensão das minhas ausências, pelo incentivo e por sempre acreditarem em mim.

Ao Carlos, por estar sempre presente nos momentos da minha vida, pelo carinho e incentivo ao meu ingresso na pós-graduação.

À CAPES pelo apoio financeiro.

Aos professores e funcionários da pós graduação e a todos que direta ou indiretamente contribuíram para a realização deste trabalho. 


\section{Resumo}

Nesta dissertação de mestrado, apresentamos um estudo dos modelos de séries temporais com componentes sazonais, tais que a medida presente está correlacionada com a medida imediatamente passada e com médias passadas no mesmo ponto de períodos anteriores. Dentro da classe de modelos periódicos, vamos considerar os modelos auto-regressivos periódicos - PAR. Estes modelos são adequados quando a correlação entre os meses variam de forma periódica, estas séries são ditas periodicamente estacionárias.

$\mathrm{Na}$ análise Clássica a identificação do modelo é feita através da função de autocorrelação periódica, PeFAC e função de autocorrelação parcial periódica, PeFACP, a escolha do melhor modelo é feita usando-se o Critério de Informação Bayesiano, BIC, apresentamos ainda um teste estatístico para verificar a periodicidade na função de autocorrelação.

$\mathrm{Na}$ análise Bayesiana consideramos três alternativas de densidades a priori para os parâmetros. A densidade preditiva é usada na escolha do melhor modelo e para fazer previsões um passo a frente de valores futuros da série usando resultados da simulaçãio em Cadeia de Markov, MCMC. Exploramos ainda o uso dos algoritmos de MCMC para estimar as densidade a posteriori marginais dos parâmetros do modelo.

A metodologia desenvolvida neste trabalho é exemplificada com conjuntos de dados reais e simulados. 


\section{Abstract}

In this work we present a Bayesian approch seasonal time series using periodical autoregressive models PAR. In the Classical model order was estimated by periodical autocorrelation PeACF and periodical partial autocorrelation funcition PePACF. The parsimonious model was chosen by Bayesian Information Criterios (BIC). In this work we present also a test of hypothesis for periodicity of the autocorrelation function.

The Bayesian approach in this work was made using three priori density function, NonInformative priori, conjugate priori Normal-Gama and $t$-Student times Gama priori. The forecast was made by simulation of Monte Carlo Markow Chain. For model selection we could consider the predictive for the futures values. 


\section{Índice}

Capítulo 1. Introdução..............................................................................................

1.1. Objetivos da Pesquisa...................................................................... 03

1.2. Organização do Trabalho .................................................................. 05

Capítulo 2. Análise Clássica para Modelos PAR................................................

2.1. Descrição do Modelo........................................................................... 07

2.2. Funções Periódicas.............................................................................. 09

2.2.1. Função de Autocorrelą̧ão Periódica, PeFAC......................... 10

2.3. Função de Autocorrelação Parcial Periódica, PeFACP.......................... 17

2.3.1. Estimativas preliminares dos parâmetros............................... 20

2.4. Função de Verossimilhança................................................................ 20

2.41. Estimadores de Máxima Verossimilhança............................ 23

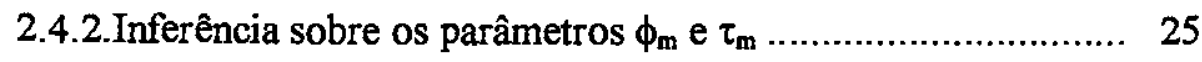

2.5. Identificação da Ordem do Modelo....................................................... 26

Capítulo 3. Análise Bayesiana para Modelos PAR............................................. 28

3.1 Densidade a Priori Não-Informativa................................................... 30

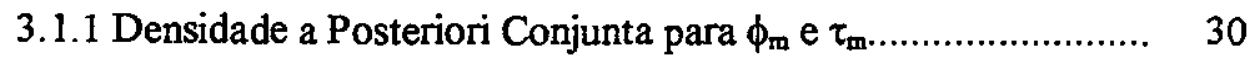

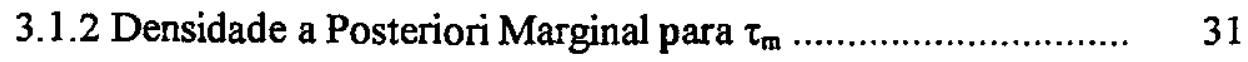

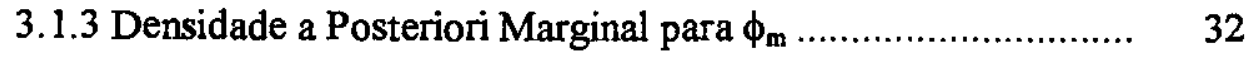

3.1.4 Densidades a Posteriori Condicionais para $\tau_{\mathrm{m}}$ e $\phi_{\mathrm{m}} \ldots \ldots \ldots \ldots \ldots . . . . . .33$

3.2 Densidades a Priori Informativas............................................................ 34

3.3 Densidade a Priori Normal - Gama....................................................... $\quad 34$

3.3.1 Densidade a Posteriori Conjunta para $\phi_{\mathrm{m}}$ e $\tau_{\mathrm{m}} \ldots \ldots \ldots \ldots \ldots \ldots \ldots \ldots . . . . . . . . . .35$

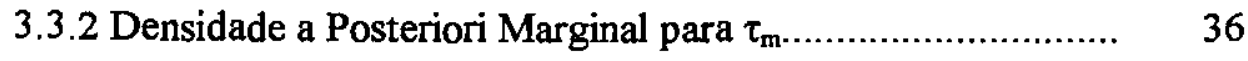

3.3.3 Densidade a Posteriori Marginal para $\phi_{\mathrm{m} \ldots \ldots \ldots \ldots \ldots \ldots \ldots \ldots \ldots \ldots . . .} \quad 36$ 
3.3.4 Densidades a Posteriori Condicionais para $\tau_{\mathrm{m}} \mathrm{e} \phi_{\mathrm{m}} \ldots \ldots \ldots \ldots \ldots \ldots . . . . . .38$

3.4 Densidade a Priori $t$-Student para $\phi_{\mathrm{m}}$ e Priori Gama para $\tau_{\mathrm{m}} \ldots \ldots \ldots \ldots \ldots \ldots \ldots . . . . . . . . .39$

3.4.1 Densidade a Posteriori Conjunta para $\phi_{m}$ e $\tau_{m} \ldots \ldots \ldots \ldots \ldots \ldots . . . . . . . . . .39$

3.4.2 Densidades a Posteriori Condicionais para $\tau_{\mathrm{m}}$ e $\phi_{\mathrm{m}} \ldots \ldots \ldots \ldots \ldots . . . . . .40$

Capítulo 4. Densidade Preditiva.......................................................................... 42

4.1 Densidade Preditiva um Passo a Frente .................................................. 42

4.2 Seleção de Modelos...................................................................... 45

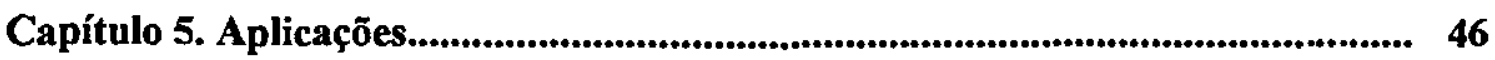

5.1 Aplicação do Teste de Sazonalidade ................................................... 36

5.2 Aplicą̧ões para o Exemplo 5 ............................................................ 52

5.3 Aplicą̧⿸丆es para o Exemplo 6 .............................................................. 73

5.4 Aplicações para o Exemplo 3 .............................................................. 93

5.5 Previsões dos Valores Futuros......................................................... 112

Capitulo 6. Conclusões e Perspectivas Futuras.................................................... 115

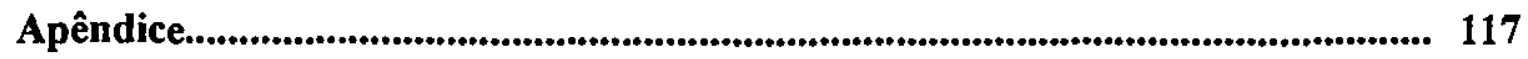

A. $\mathrm{O}$ algoritmo amostrador de Gibbs............................................................ 117

B. Algoritmo de Metropolis - Hastings............................................................ 118

C. Monitoração Informal da Convergência.......................................................... 119

D. Verificação Formal da Convergência ............................................................. 120

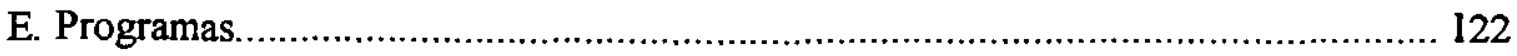

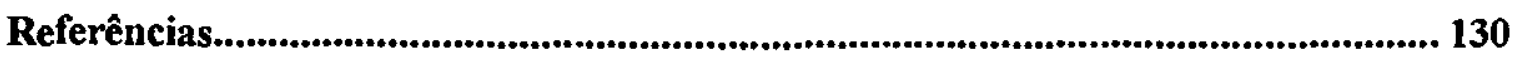




\section{CAPÍTULO 1}

\section{Introdução aos Modelos de Séries Temporais ·}

A análise estatística de modelos de séries temporais tem aplicações em diversas áreas, tais como, engenharia, econômia e outras. Os modelos de séries temporais do tipo AR (auto-regressivo) são bem conhecidos e considerados adequados para modelar séries estacionárias. No entanto, nem todos os conjuntos de dados que constituem uma série temporal podem ser modelados por modelos AR. Em muitas situações práticas encontramos séries temporais que apresentam um comportamento sazonal. Em geral, dizemos que uma série apresenta um comportamento sazonal com período $s$, quando ocorrem similaridades na série após $s$ intervalos de tempo. Para ilustrar, consideramos alguns exemplos que caracterizam séries sazonais. A Figura 1(a) mostra o número total de passageiros em uma companhia aérea internacional para 1952 a 1954 [Box, Jenkins e Reinsel-1994], a Figura 1(b) mostra o consumo de energia elétrica no setor industrial (MWh/1000) para 1975 a 1986 [Andrade, Carvalho e Barone-1987], a Figura 1(c) mostra valores mensais de concentração de ozônio (em partes por 100 milhões) em Azuza, Califórnia para 1956 a 1970 e ą Figura 1(d) mostra uma série de 10 anos de vazões médias mensais em $\left(\mathrm{m}^{3} / \mathrm{s}\right)$ que chegam ao reservatório da hidroelétrica de Furnas, no Sudeste do Brasil para os anos de 1980 a 1990 (dados fornecidos pela eletrobrás para os anos de 1931 a 1990). 


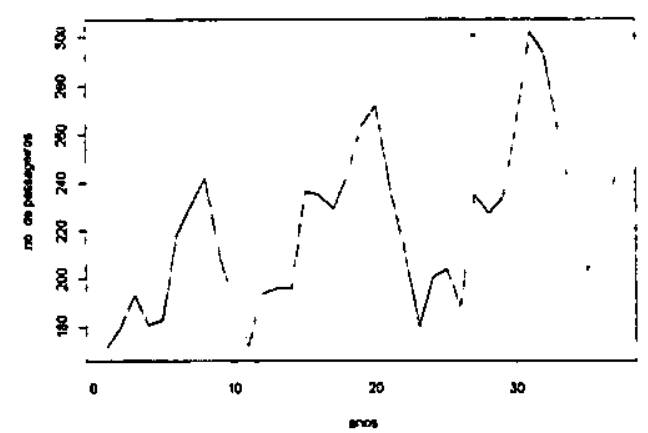

(a)

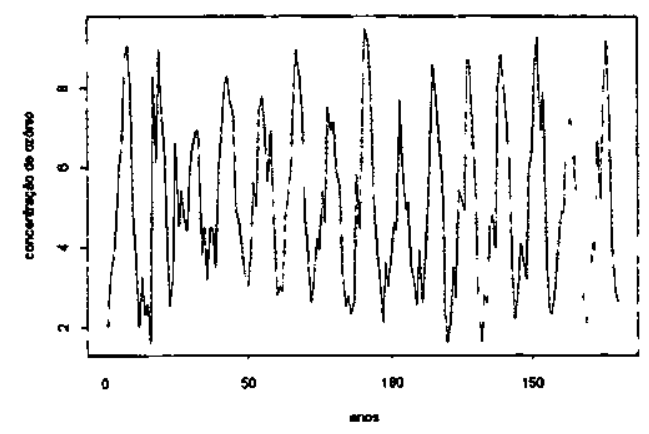

(c)

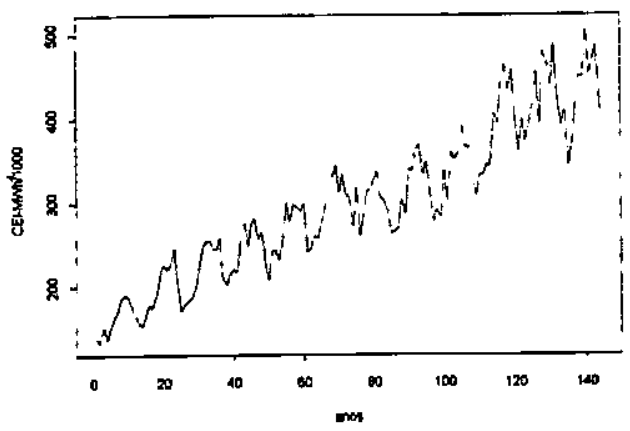

(b)

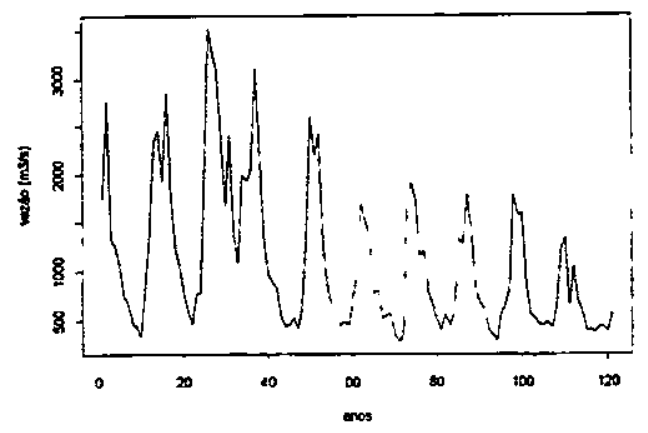

(d)

Figura 1(a): Série do número total de passageiros em uma companhia aérea internacional; (b): Série do consumo de energia elétrica no setor industrial; (c): Série da concentração de ozônio; (d): Série de vazões em Furnas.

Para séries sazonais, onde o valor observado no instante $t$ está correlacionado com os valores passados no instante $t-s$ ( $s$ é o período) é mais adequado ajustar um modelo cujos parâmetros também variam de uma forma periódica do que um modelo com parâmetros constantes para todos os meses. Então, quando a correlação entre os meses variam de forma periódica, estas séries são ditas perio dicamente estacionárias. Dentro da classe dos modelos periódicos, consideramos 
os modelos auto-regressivos periódicos (PAR).

Estes modelos foram inicialmente propostos por Jones e Breslford (1967) e posteriormente estudados por Vecchia (1985) e Anderson e Vecchia (1993). A estimação de parâmetros apresenta mais dificuldades em modelos PAR que nos modelos comuns AR, devido ao grande número de parâmetros a serem estimados. Este problema é abordado em Vecchia (1993) que apresenta um algoritmo para encontrar estimativas de máxima verossimilhança para modelos PAR. Adams e Goodwin (1995), propõem um método iterativo para cálculo dos estimadores de máxima verossimilhança dos parâmetros de modelos ARMA periódicos.

O esforço computacional para encontrar estimativas de máxima verossimi lhança de modelos PAR é geralmente muito grande, porém, recentemente técnicas Bayesianas tornaram-se uma alternativa atrativa para a metodologia de Box-Jenkins. Uma das vantagens do uso de métodos Bayesianos é permitir a consideração de informações a priori sobre os parâmetros do modelo na análise. Além disso, uma vantagem dos métodos Bayesianos é o uso de técnicas de simulação de Monte Carlo em Cadeia de Markov, MCMC [Gelfand e Smith (1990a ; 1990b)] para fazer inferência de parâmetros em modelos complexos.

É neste contexto que definimos os objetivos desse trabalho.

\subsection{Objetivos da Pesquisa}

O objetivo do presente trabalho é utilizar a abordagem Bayesiana para a identificação, estimação e previsão dos modelos PAR. A análise Bayesiana para esses 
modelos tem a finalidade de obter resultados mais precisos que a análise de verossimi lhança, ou seja, obter estimativas melhores, pois nos casos de séries temporais geralmente temos amostras pequenas, para ajuste de modelos PAR. Por exemplo, considerando uma série de observações mensais feitas por 10 anos, temos uma série com 120 observações. No entanto, se essa série é sazonal com período $s=12$, temos que ajustar 12 modelos (um para cada mês), assim temos somente 10 observações para cada modelo.

Considerando as informações adicionais a priori para os parâmetros do modelo na análise esperamos obter melhores estimativas para esses parâmetros.

Neste trabalho, a análise Bayesiana dos modelos auto-regressivos periódicos é feita considerando três densidades a priori diferentes, primeiramente consideramos a priori Não-Informativa de Jeffreys, onde as densidades a posteriori marginal, para os parâmetros do modelo, podem ser calculadas analiticamente e verificamos que o valor esperado dessa posteriori coincide com o valor do estimador de máxima verossimilhança. No segundo caso, adotamos uma priori conjugada Normal-Gama, onde as densidade a posteriori também podem se calculadas analiticamente, resultando em uma densidade $t$-Student para os parâmetros do modelo e uma densidade Gama para o inverso da variância dos resíduos. A análise da expressão do estimador de Bayes, para os parâmetros do modelo, mostra que este estimador é uma média ponderada entre o esimador de máxima verossimilhança e o valor esperado da densidade a priori.

O uso de densidades a priori conjugadas, em geral, recebem muitas críticas por 
serem funções pouco realistas para tratamento de problemas reais. Para contornar essas críticas adotamos no terceiro caso uma densidade a priori informativa dada pelo produto de uma densidade $t$ - Student e uma densidade Gama, resultando em uma densidade a posteriori não-padronizada. Neste caso, a análise a posteriori é feita usando-se algoritmos de simulação em cadeia de Markov (MCMC), do tipo Gibbs Sampling [Casella e George-1992] e Metropolis-Hastings [Chib e Greenberg1995], os quais vêm se mostrando satisfatórios do ponto de vista de precisão e esforços computacionais.

Um critério de seleção de modelos baseado na densidade preditiva é aplicado. Esses resultados são comparados com a análise clássica onde a identificação do modelo é feita através da função de autocorrelação periódica, PeFAC e função de autocorrelação parcial periódica, PeFACP, a escolha do melhor modelo é feita usando-se o Critério de Informação Bayesiano, BIC [Schwarz-1978].

Neste trabalho apresentamos ainda um teste estatístico para avaliar a hipótese nula $H_{0}$ de que a PeFAC do processo não é periódica, esse teste é baseado nas propriedades assintóticas dos coeficientes da PeFAC e estabelece um nível de confiança ao rejeitar $H_{0}$.

\subsection{Organização do Trabalho}

O presente trabalho está organizado em 6 capítulos. Este capítulo introdutório apresenta os modelos de séries temporais sazonais considerados nesta dissertação e descreve os objetivos a serem atingidos. 
No capítulo 2, apresentamos sob o enfoque da teoria assintótica, as inferências estatísticas para os parâmetros do modelo PAR e teste de sazonalidade da função de autocorrelação.

No capítulo 3, apresentamos uma análise Bayesiana, considerando a densidade a priori Não-Informatiava de Jeffreys [Box e Tiao-1973] e as densidades a priori informativas conjugada Normal-Gama e $t$-Student versus Gama respectivamente. Algoritmos computacionais, como amostrador de Gibbs e Metropolis-Hastings são utilizados.

No capítulo 4, deduzimos a densidade preditiva um passo a frente, esta densidade preditiva é utilizada para fazer previsões de valores futuros da série usando resultados da simulação MCMC.

Exemplos de aplicação das metodologias para vários conjuntos de dados são apre sentados no capítulo 5 e finalmente no capítulo 6 são apresentadas as conclusões finais e comentários sobre perspectivas futuras.

No apêndice, são apresentados os algoritmos amostrador de Gibbs, MetropolisHastings e a monitoração informal e a verificação formal da convergência de Gelman e Rubin (1992). 


\section{CAPÍTULO 2}

\section{Análise Clássica para Modelos PAR}

Neste capítulo, descrevemos os modelos auto-regressivos periódicos - $\operatorname{PAR}\left(p_{m}\right)$ e apresentamos uma análise clássica para este modelo, considerando algumas propriedades assintóticas obtidas através do método de máxima verossimilhança, empregado em inferência clássica.

\subsection{Descrição do Modelo}

Sejam $\tilde{Z}_{t}, t=1, \ldots, n s$, observações consecutivas de uma série temporal periódica com período $s$, (quando $s=12$, trata-se de uma série mensal com período de 12 meses e $n$ representa o número de anos). Podemos escrever o índice de tempo $t$ como uma função do número de anos $r=1, \ldots, n$ e dos meses $m=1, \ldots, s$, ou seja, $t=t(r, m)=(r-1) s+m$. Assim temos, $\tilde{Z}_{t(r, m)}$ como sendo a observação da série $\tilde{Z}_{t}$ no mês $m$ do ano $r$.

Definimos a média $\mu_{t(r, m)}=E\left(\tilde{Z}_{t(r, m)}\right)$ e a função de autocovariância $\gamma_{t(r, m)}(j)=$ $\operatorname{cov}\left(\tilde{Z}_{t(r, m)}, \tilde{Z}_{t(r, m)+j}\right)$, então, dizemos que o processo $\left\{\tilde{Z}_{t(r, m)}, r=1, \ldots, n, m=\right.$ $1, \ldots, s\}$ é periodicamente estacionário com período $s$ se para dois números quaisquer $j$ e $k$, temos:

$$
\mu_{t(r, m)}=\mu_{t(r, m)+k s}
$$




$$
\gamma_{t(r, m)}(j)=\gamma_{t(r, m)+k s}(j)
$$

onde $\gamma_{t(r, m)}(0)=\sigma_{m}^{2}$ é a variância do processo no mês $m$.

As equações (2.1) e (2.2) nos permite escrever $\mu_{t(r, m)}$ e $\gamma_{t(r, m)}(j)$ como função só de $m$. Para $r=1$, temos, $t(1, m)=m$ e $t(1, m)+k s=m+k s$, então,

$$
\begin{aligned}
\mu_{m} & =\mu_{m+k s}, \\
\sigma_{m}^{2} & =\sigma_{m+k s}^{2}, \\
\gamma_{m}(j) & =\gamma_{m+k s}(j) .
\end{aligned}
$$

Para $r \neq 1, t(r, m)=(r-1) s+m+k s=m+k^{\prime} s$, onde $\mathrm{k}^{\prime}=r+k-1$, assim,

$$
\begin{aligned}
\mu_{m} & =\mu_{m+k^{\prime} s} \\
\sigma_{m}^{2} & =\sigma_{m+k^{\prime} s}^{2} \\
\gamma_{m}(j) & =\gamma_{m+k^{\prime} s}(j) .
\end{aligned}
$$

Neste trabalho, sempre que estivermos nos referindo a um parâmetro que varia de forma periódica no tempo vamos indexá-lo somente no índice $m$, ou seja, o parâmetro se refere ao mês do ano. Assim temos por exemplo:

$$
\begin{aligned}
\mu_{t(r, m)} & =\mu_{t(l, m)}=\mu_{m}, \\
\sigma_{t(r, m)}^{2} & =\sigma_{t(l, m)}^{2}=\sigma_{m}^{2}, \\
\gamma_{t(r, m)}(j) & =\gamma_{t(l, m)}(j)=\gamma_{m}(j) .
\end{aligned}
$$


Uma classe de modelos usuais para descrever séries temporais periodicamente estacionárias são os modelos auto-regressivos periódicos $\operatorname{PAR}\left(p_{m}\right)$ de ordem $p_{m}, m=$ $1, \ldots, s$, estes modelos são dados por:

$$
Z_{t(r, m)}=\sum_{i=1}^{p_{m}} \phi_{i, m} Z_{t(r, m)-i}+a_{t(r, m)}, \quad \quad t(r, m) \geq p_{m}+1
$$

onde $Z_{t(r, m)}$ representa a série padronizada,

$$
Z_{t(r, m)}=\frac{\tilde{Z}_{t(r, m)}-\mu_{m}}{\sigma_{m}}
$$

e $\mu_{m}$ e $\phi_{i, m}$ são funções periódicas com período $s$ e $a_{t(r, m)}$ é ruído gaussiano com as seguintes propriedades:

$$
\begin{gathered}
E\left(a_{t(r, m)}\right)=0 \\
E\left(a_{t(r, m)}, a_{t(r, m)+j}\right)=\left\{\begin{array}{cc}
\tau_{m}^{-1} & , j=0 \\
0 & , j \neq 0
\end{array}\right.
\end{gathered}
$$

onde $\tau_{m}^{-1}=\sigma_{m}^{2}, \tau_{m}>0$.

\subsection{Funções Periódicas}

Nesta seção, apresentamos as funções periódicas de autocorrelação e autocorrelação parcial. A função de autocorrelação periódica, PeFAC identifica graficamente a presença de correlações periódicas entre os meses e a função de autocorrelação parcial periódica, $\mathrm{PeFACP}$, determina também graficamente a ordem do modelo, ordem esta que será confirmada através do Critério de Informação Bayesiano definido em (2.47). 


\subsubsection{Função de Autocorrelação Periódica, PeFAC}

Vamos estabelecer as propriedades assintóticas do estimador das funções de autocorrelações $\rho_{m}(j)$ para vários passos $j$. O comportamento assintótico para o estimador da função de autocorrelações $\widehat{\rho}_{m}(j)$ para dois passos fixos $j, k$ e para $m=1, \ldots, s$.

O estimador da função de autocorrelação é dado por:

$$
\widehat{\rho}_{m}(j)=\frac{\widehat{\gamma}_{m}(j)}{\left[\widehat{\gamma}_{m}(0) \widehat{\gamma}_{m+j}(0)\right]^{1 / 2}}
$$

onde a covariância estimada com base nos dados $\left\{\tilde{Z}_{t(r, m)}, r=1, \ldots, n, m=\right.$ $1, \ldots, s\}$ é definida como:

$$
\widehat{\gamma}_{m}(j)=\frac{1}{n} \sum_{r=1}^{n}\left(\tilde{Z}_{t(r, m)}-\widehat{\mu}_{m}\right)\left(\tilde{Z}_{t(r, m)+j}-\widehat{\mu}_{m+j}\right)
$$

onde

$$
\widehat{\mu}_{m}=\frac{1}{n} \sum_{r=1}^{n} \widetilde{Z}_{t(r, m)}
$$

Os termos na equação $(2.5)$ envolvendo $\tilde{Z}_{t(r, m)}$, são adotados iguais a zero sempre que $t(r, m)>n s$.

A distribuição conjunta das autocorrelações periódicas estimadas é dada no Teorema abaixo, o qual foi provado por Anderson e Vecchia (1993).

Teorema: Seja $Z_{t(r, m)}=\widetilde{Z}_{t(r, m)}-\mu_{m}$ onde $\left\{Z_{t(r, m)}, r=1, \ldots, n, m=1, \ldots, s\right\}$ é um processo auto-regressivo periódico e $\mu_{m}$ é uma função perí́dica com período s. Então para quaisquer positivos $j$ e $k \operatorname{com} j \neq k$, 


$$
n^{1 / 2}\left[\begin{array}{c}
\hat{\rho}_{m}(j)-\rho_{m}(j) \\
\hat{\rho}_{m}(k)-\rho_{m}(k)
\end{array}\right] \Rightarrow N\left(0,\left[\begin{array}{cc}
W_{j j} & W_{j k} \\
W_{k j} & W_{k k}
\end{array}\right]\right)
$$

onde' $\Rightarrow^{\prime}$ denota convergência em distribuição, $N(\boldsymbol{\mu}, W)$ denota a distribuição normal multivariada com vetor de média $\mu$ e matriz de covariância $W$.

$$
\begin{aligned}
& \hat{\rho}_{m}(l)=\left[\rho_{1}(l), \rho_{2}(l), \ldots, \rho_{s}(l)\right]^{\prime} \\
& \rho_{m}(l)=\left[\rho_{1}(l), \rho_{2}(l), \ldots, \rho_{s}(l)\right]^{\prime}
\end{aligned}
$$

e

$$
\begin{aligned}
W_{j k}= & \sum_{v=-\infty}^{+\infty}\left\{F_{v} \pi^{j} F_{v+k-j} \pi^{v-j}+F_{v+k} \pi^{j} F_{v-j} \pi^{v-j}-F_{j}\left(I+\pi^{j}\right) F_{v} F_{v+k} \pi^{v}-\right. \\
& \left.-F_{v} \pi^{j} F_{v-j} \pi^{v-j}\left(I+\pi^{-k}\right) F_{k}+\frac{1}{2} F_{j}\left(I+\pi^{j}\right) F_{v}^{2} \pi^{v}\left(I+\pi^{-k}\right) F_{k}\right\}
\end{aligned}
$$

onde $I$ é a matriz identidade $s \times s$ e $F_{v}=\operatorname{diag}\left\{\rho_{1}(v), \rho_{2}(v), \ldots, \rho_{s}(v)\right\}$ e $\pi$ é uma matriz ortogonal de permutação cíclica $s \times s$ :

$$
\pi=\left[\begin{array}{cccccc}
0 & 1 & 0 & 0 & \cdots & 0 \\
0 & 0 & 1 & 0 & \cdots & 0 \\
\vdots & \vdots & \vdots & \vdots & \ddots & \vdots \\
0 & 0 & 0 & 0 & \cdots & 1 \\
1 & 0 & 0 & 0 & \cdots & 0
\end{array}\right]
$$

com $\pi^{-k} \equiv\left(\pi^{\prime}\right)^{k}, \pi^{0}$ a matriz de identidade $s \times s \mathrm{e}$,

$$
\pi^{j} \operatorname{diag}\left\{a_{1}, a_{2}, \ldots, a_{s}\right\} \pi^{-j}=\operatorname{diag}\left\{a_{j}, a_{j+1}, \ldots, a_{j-s}\right\}
$$


onde $\left\{a_{m}\right\}$ é qualquer sequência de constantes satisfazendo $a_{m+k s}=a_{m}$ para todo $k$.

Quando $s=1$, a expressão para $W_{j k}$ em (2.9) reduz-se a:

$$
\begin{aligned}
W_{j k}= & \sum_{v=-\infty}^{+\infty}\{\rho(v) \rho(v+k-j)+\rho(v+k) \rho(v-j)-2 \rho(j) \rho(v) \rho(v+k)- \\
& \left.-2 \rho(v) \rho(v-j) \rho(k)+2 \rho(j) \rho(k) \rho^{2}(v)\right\}
\end{aligned}
$$

considerando agora $j=k$ temos a variância de $n^{1 / 2}\left[\hat{\rho}_{m}(j)-\rho_{m}(j)\right]$ a qual é muito útil na identificação da ordem dos modelos. Essa variância é dada por:

$$
\begin{aligned}
W_{k k}= & \sum_{v=-\infty}^{+\infty}\left\{\rho^{2}(v)+\rho(v+k) \rho(v-k)-2 \rho(k) \rho(v) \rho(v+k)-\right. \\
& \left.-2 \rho(v) \rho(v-k) \rho(k)+2 \rho^{2}(k) \rho^{2}(v)\right\}
\end{aligned}
$$

A equação (2.11) é conhecida como fórmula de Bartlett para processos estacionários de segunda ordem [Bartlett-1946 apud Anderson e Vecchia-1993].

Supondo agora um processo para o qual todas as autocorrelações $\rho(v)=0$ para $v>q$, então todos os termos de (2.11), exceto o primeiro são zero para $k>q$. Assim a variância de $n^{1 / 2}\left[\widehat{\rho}_{m}(j)-\rho_{m}(j)\right]$ é dada por:

$$
W_{k k}=\left\{1+2 \sum_{v=1}^{q} \rho^{2}(v)\right\}
$$

A expressão (2.12) é muito útil quando a função de autocorrelação do processo é usada para identificar a ordem dos modelos. Isto é feito testando-se a hipótese nula $H_{0}: \rho(j)=0$ e construindo-se o intervalo de confiança para $\rho(j)$ dado por: 


$$
\widehat{\rho}(j) \pm z_{\alpha} \sqrt{\operatorname{Var}(\hat{\rho}(j))}
$$

onde $z_{\alpha}$ é uma estatística $N(0,1)$ e $\sqrt{\operatorname{Var}(\hat{\rho}(j))} \approx \frac{1}{n}\left\{1+2 \sum_{v=1}^{q} \rho^{2}(v)\right\}$.

\section{Determinaçāo da Periodicidade na Funçāo de Autocorrelaçāo}

Supondo que $\left\{Z_{t(r, m)}\right\}$ é uma série temporal sazonal observada sobre s-períodos, é importante decidir se $\rho_{m}(j)$ pode ser considerado constante ou se $\rho_{m}(j)$ varia periodicamente. Assumir que $\rho_{m}(j)$ é constante é equivalente a assumir que o processo $\left\{\tilde{Z}_{t(r, m)}-\mu_{m}\right\}$ é estacionário no sentido fraco [Box; Jenkins; Reinsel1994]. Essa suposição resulta em consideráveis simplificações na inferência dos parâmetros do modelo. Entretanto, assumir falsamente que $\rho_{m}(j)$ é constante pode levar a sérios erros na inferência [Tiao; Grupe-1980].

Nesta seção, apresentaremos um método para testar a hipótese de que $\rho_{m}(j)$ é constante em $m$ versus a hipótese alternativa de que $\rho_{m}(j)$ varia periodicamente com período $s$.

A estatística teste é obtida a partir da representação de $\rho_{m}(j), m=1, \ldots, s$ em série de Forrier:

$$
\rho_{m}(j)=\alpha_{0}+\sum_{i=1}^{s / 2} \alpha_{i} c_{i m}+\beta_{i} s_{i m}
$$

Consideramos somente os casos onde $s$ é par, ou mais frequentemente $s=12$ meses. Assim temos, $c_{i m}=\cos 2 \pi f_{i m}, s_{i m}=\operatorname{sen} 2 \pi f_{i m} \in f_{i}=i / s$ é a frequência do i-ésimo hamônico cujo harmônico fundamental tem frequência $f_{1}=1 / s$ ( quando 
$\left.s=12, f_{1}=1 / 12\right)$. Os estimadores de mínimos quadrados dos coeficientes $\alpha_{0} \mathrm{e}$ $\left(\alpha_{i}, \beta_{i}\right)$ são dados por:

$$
\begin{gathered}
a_{i}=\frac{1}{s} \sum_{m=1}^{s} \hat{\rho}_{m}(j) c_{i m}, i=0, \frac{s}{2} \\
a_{i}=\frac{2}{s} \sum_{m=1}^{s} \hat{\rho}_{m}(j) c_{i m}, i=1,2, \ldots, \frac{s}{2}-1 \\
b_{i}=\frac{2}{s} \sum_{m=1}^{s} \hat{\rho}_{m}(j) s_{i \pi n}, i=1,2, \ldots, \frac{s}{2}-1
\end{gathered}
$$

onde $b_{s / 2}=0$ e $a_{0}$ é a média dos $\widehat{\rho}_{m}(j)$, ou seja,

$$
a_{0}=\frac{1}{s} \sum_{m=1}^{s} \hat{\rho}_{m}(j)
$$

Sob a hipótese $H_{0}$ de que $\rho_{m}(j)$ é constante e não depende de $m$, temos $a_{0}=$ $\hat{\rho}_{m}(j)=\widehat{\rho}(j)$, assim,

$$
\widehat{\rho}(j)-\rho(j)=\sum_{i=1}^{s / 2}\left(a_{i} c_{i m}+b_{i} s_{i m}\right)
$$

sabemos que $\widehat{\rho}(j)-\rho(j)$, tem distribuição normal com média zero, portanto,

$$
E(\widehat{\rho}(j)-\rho(j))=0
$$

substituindo (2.19) em (2.20) temos:

$$
E\left(a_{i}\right)=E\left(b_{i}\right)=0, i=1,2, \ldots, q
$$


As variâncias $\operatorname{Var}\left(a_{i}\right)$ e $\operatorname{Var}\left(b_{i}\right)$ podem ser calculadas por $E\left(a_{i}^{2}\right), E\left(b_{i}^{2}\right)$ respectivamente, usando $(2.16)$ e $(2.17)$ e supondo $\widehat{\rho}_{m}(j)=\widehat{\rho}(j)$, ou seja, sob $H_{0}$ temos,

$$
\operatorname{Var}\left(a_{i}\right)=\frac{4 \operatorname{Var}(\widehat{\rho}(j))}{s^{2}} \sum_{i=1}^{s} c_{i m}^{2}=\frac{4 \operatorname{Var}(\widehat{\rho}(j))}{s^{2}}, i=1,2, \ldots, \frac{s}{2}-1
$$

similarmente para $b_{i}$ temos,

$$
\operatorname{Var}\left(b_{i}\right)=\frac{4 \operatorname{Var}(\widehat{\rho}(j))}{s^{2}} \sum_{i=1}^{s} s_{i m}^{2}=\frac{4 \operatorname{Var}(\hat{\rho}(j))}{s^{2}}, i=1,2, \ldots, \frac{s}{2}-1
$$

podemos calcular ainda

$$
\operatorname{Cov}\left(a_{i}, b_{i}\right)=\frac{4 \operatorname{Var}(\widehat{\rho}(j))}{s^{2}} \sum_{i=1}^{s / 2} c_{i m} s_{i m}=0
$$

Sendo $a_{i}$ e $b_{i}$ funções lineares de variáveis aleatórias normais, então $a_{i}$ e $b_{i}$ também são variáveis aleatórias normais. Desta forma, podemos definir as variáveis aleatórias:

$$
\begin{gathered}
\frac{a_{i}^{2}}{\operatorname{Var}\left(a_{i}\right)}=\frac{s a_{i}}{4 \operatorname{Var}(\widehat{\rho}(j))}, i=1,2, \ldots, \frac{s}{2} \\
\frac{b_{i}^{2}}{\operatorname{Var}\left(b_{i}\right)}=\frac{s b_{i}}{4 \operatorname{Var}(\widehat{\rho}(j))}, i=1,2, \ldots, \frac{s}{2}-1
\end{gathered}
$$

Então (2.24) e (2.25) definem-se $s-1$ variáveis aleatórias com distribuição qui-quadrado com grau de liberdade igual a um. Sendo $a_{i}$ e $b_{i}$ variáveis aleatórias normais independentes podemos definir a variável: 


$$
\chi_{s-1}^{2}=\sum_{i=1}^{s / 2-1}\left[\frac{a_{i}^{2}}{\operatorname{Var}\left(a_{i}\right)}+\frac{b_{i}^{2}}{\operatorname{Var}\left(b_{i}\right)}\right]+\frac{a_{q}^{2}}{\operatorname{Var}\left(a_{q}\right)}
$$

onde $\chi_{s-1}^{2}$ é uma variável aleatória qui-quadrado com $s-1$ graus de liberdade. Usando $(2.20)$ e (2.21) temos,

$$
\chi_{s-1}^{2}=\frac{s}{4 \operatorname{Var}(\widehat{\rho}(j))} \sum_{i=1}^{s / 2-1}\left(a_{i}^{2}+b_{i}^{2}\right)+\frac{s}{\operatorname{Var}(\widehat{\rho}(j))} a_{q}^{2}
$$

Um teste para a hipótese que $\rho_{m}(j)$ é constante em $m$ pode ser construído baseado na estatística $\chi_{s-1}^{2}$, onde com um nível de significância $\alpha$ para o erro tipo I, comparamos $\chi_{s-1}^{2}$ calculada em (2.27) com o valor crítico $\chi_{s-1}^{2}(\alpha)$, ou seja, rejeitamos a hipótese $H_{0}$, ao nível $\alpha$ se $\chi_{s-1}^{2}>\chi_{s-1}^{2}(\alpha)$. Neste caso, uma estrutura de autocorrelação periódica é indicada. Esse método é similar ao proposto por Vecchia e Ballerini (1991), foram expressas em forma da densidade espectral de vários produtos de processos $\left\{Z_{t(r, m)} Z_{t(r, m)}\right\}$. Em Anderson e Vecchia (1993), eles mostram que expressar a variância assintótica diretamente em termos da função de autocorrelação aumenta o poder do teste. Neste trabalho, seguimos a proposta de Anderson e Vecchia (1993), no entanto, a variância assintótica é estimada usando a aproximação de Bartlett a qual significa consideravelmente a estimação da variância e um menor esforço computtacional, aumentando a viabilidade da aplicação do teste em problemas práticus, Vários exemplos serão apresentados mais adiante no capítulo Aplicações. 


\subsection{Função de Autocorrelação Parcial Periódica, PeFACP}

Inicialmente não podemos saber qual a ordem do processo auto-regressivo periódico para o ajuste das observações da série temporal. A função de autocorrelação parcial periódica é um esquema o qual explora o fato de considerando um processo auto-regressivo periódico temos uma função de autocorrelação a qual é infinita em extensão, isto é, a função de autocorrelação parcial periódica descreve os termos de $p_{m}$ diferentes de zero.

Denotamos por $\phi_{i, m}$ o i-ésimo coeficiente de um modelo $\operatorname{PAR}\left(p_{m}\right)$ para o $m$-ésimo mês, de tal modo que $\phi_{p_{m}, m}$ seja o último coeficiente. Então para $i=1, \ldots, p_{m}$, temos a função de autocorrelação dada por

$$
\rho_{m}(j)=\phi_{1, m} \rho_{m}(j-1)+\phi_{2, m} \rho_{m}(j-2)+\ldots+\phi_{p_{m}, m} \rho_{m}\left(j-p_{m}\right) \dot{j}>0
$$

se fizermos $j=1, \ldots, p_{m}$ em (2.28) obtemos

$$
\begin{gathered}
\rho_{m}(1)=\phi_{1, m}+\phi_{2, m} \rho_{m}(1)+\ldots+\phi_{p_{m}, m} \rho_{m}\left(p_{m}-1\right) \\
\rho_{m}(2)=\phi_{1, m} \rho_{m}(1)+\phi_{2, m}+\ldots+\phi_{p_{m}, m} \rho_{m}\left(p_{m}-2\right) \\
\vdots \\
\rho_{m}\left(p_{m}\right)=\phi_{1, m} \rho_{m}\left(p_{m}-1\right)+\phi_{2, m} \rho_{m}\left(p_{m}-2\right)+\ldots+\phi_{p_{m}, m}
\end{gathered}
$$

que são denominadas equações de Yule-Walker. Em forma matricial, podemos 
escrever

$\left[\begin{array}{ccccc}1 & \rho_{m}(1) & \rho_{m}(2) & \ldots & \rho_{m}\left(p_{m}-1\right) \\ \rho_{m}(1) & 1 & \rho_{m}(1) & \ldots & \rho_{m}\left(p_{m}-2\right) \\ \vdots & \vdots & \vdots & \vdots & \vdots \\ \rho_{m}\left(p_{m}-1\right) & \rho_{m}\left(p_{m}-2\right) & \rho_{m}\left(p_{m}-3\right) & \ldots & 1\end{array}\right]\left[\begin{array}{c}\phi_{1, m} \\ \phi_{2, m} \\ \vdots \\ \phi_{p_{m}, m}\end{array}\right]=\left[\begin{array}{c}\rho_{m}(1) \\ \rho_{m}(2) \\ \vdots \\ \rho_{m}\left(p_{m}\right)\end{array}\right]$

Podemos estimar os coeficientes $\phi_{1, m}, \ldots, \phi_{p_{m}, m}$ do modelo $\operatorname{PAR}\left(p_{m}\right)$ através de (2.29) substituindo as PeFAC $\rho_{m}(j)$ por suas estimativas $\gamma_{m}(j)$.

As equações de Yule-Walker podem ser resolvidas através de um processo recurssivo, para ordens sucessivas $p_{m}=1,2,3, \ldots$, da forma

$$
\begin{gathered}
\phi_{1, m}=\rho_{m}(1) \\
\phi_{2, m}=\frac{\left|\begin{array}{cc}
1 & \rho_{m}(1) \\
\rho_{m}(1) & \rho_{m}(2)
\end{array}\right|}{\left|\begin{array}{cc}
1 & \rho_{m}(1) \\
\rho_{m}(1) & 1
\end{array}\right|}=\frac{\rho_{m}(2)-\rho_{m}^{2}(1)}{1-\rho_{m}^{2}(1)} \\
\phi_{3, m}=\frac{\left|\begin{array}{ccc}
1 & \rho_{m}(1) & \rho_{m}(1) \\
\rho_{m}(1) & 1 & \rho_{m}(2) \\
\rho_{m}(2) & \rho_{m}(1) & \rho_{m}(3)
\end{array}\right|}{\left|\begin{array}{ccc}
1 & \rho_{m}(1) & \rho_{m}(2) \\
\rho_{m}(1) & 1 & \rho_{m}(1) \\
\rho_{m}(2) & \rho_{m}(1) & 1
\end{array}\right|}
\end{gathered}
$$


e, em geral.

$$
\phi_{p_{m}, m}=\frac{\left|\rho_{m}^{*}\left(p_{m}\right)\right|}{\left|\rho_{m}\left(p_{m}\right)\right|}
$$

onde $\rho_{m}\left(p_{m}\right)$ é a matriz de autocorrelações e $\rho_{m}^{*}\left(p_{m}\right)$ é a matriz $\rho_{m}\left(p_{m}\right)$ com a última coluna substituída pelo vetor de autocorrelações. A quantidade $\phi_{p_{m}, m}$ encarada como função de $p_{m}$, denominamos função de autocorrelação parcial periódica. $\mathrm{Na}$ prática como não conhecemos as quantidades teóricas utilizamos a função de autocorrelação parcial periódica estimada, que esperamos representar adequadamente a quantidade teórica, desta forma, substituimos nas equações de Yule-Walker as $\mathrm{PeFAC}, \rho_{m}(j)$ por suas estimativas:

$$
\gamma_{m}(j)=\widehat{\phi}_{1, m} \gamma_{m}(j-1)+\ldots+\widehat{\phi}_{p_{m}, m} \gamma_{m}\left(j-p_{m}\right)
$$

e resolvemos as equações de (2.30) para $p_{m}=1,2, \ldots$ e $j=1, \ldots, p_{m}$.

Para PeFACP a variância estimada é dada por:

$$
\operatorname{Var}\left(\widehat{\phi}_{i, m}\right) \simeq \frac{1}{n}
$$

Para $n$ grande e sob a hipótese de que o processo é $\operatorname{PAR}\left(p_{m}\right), \widehat{\phi}_{i, m}$ tem distribuição aproximadamente normal com média zero e variância (2.31), de modo que o intervalo de confiança é dado por:

$$
\widehat{\phi}_{i, m} \pm \frac{2}{\sqrt{n}}
$$




\subsubsection{Estimativas preliminares dos parâmetros}

Como vimos anteriormente, com base nas autocorrelações periódicas e autocorrelações parciais periódicas estimadas, identificamos o processo $\operatorname{PAR}\left(p_{m}\right)$ resultante. Nesta seção, obteremos estimativas preliminares dos parâmetros do modelo identificado a serem usadas como valores iniciais, para o procedimento de estimação de máxima verossimilhança dos parâmetros. Estas estimativas preliminares são obtidas através das autocorrelações periódicas.

Para processos PAR $\left(p_{m}\right)$, resolvemos as equações de Yule-Walker (2.29), com $\rho_{m}(j)$ substituído por $\gamma_{m}(j)$, como mostrado pela equação (2.30).

Uma estimativa inicial para o inverso da variância residual sazonal pode ser obtida por:

$$
\widehat{\tau}_{m}=\frac{1}{c_{0, m}\left(1-\widehat{\phi}_{1, m} \gamma_{m}(1)-\widehat{\phi}_{2, m} \gamma_{m}(2)-\ldots-\widehat{\phi}_{p_{m}, m} \gamma_{m}\left(p_{m}\right)\right)}
$$

substituindo $\gamma_{m}(0)$ por $c_{0, m}$, os $\phi_{i, m}$ por suas estimativas $\widehat{\phi}_{i, m}$ e os $\rho_{m}(j)$ por suas estimativas $\gamma_{m}(j)$.

Deste modo, considendo a equação (2.30), podemos obter as estimativas de Yule-Walker para um $p_{m}$ qualquer.

\subsection{Função de Verossimilhança}

Considerando o modelo PAR definido em (2.3), denotamos $\phi_{m}$, um vetor de números reais $p_{m} \times 1$ e $\tau_{m}=\left(\tau_{1}, \ldots, \tau_{12}\right)^{\prime}$, um vetor de números reais positivos 
$m \times 1$. Para estimar $\phi_{m}$ e $\tau_{m}$ pelo método de máxima verossimilhança vamos considerar um conjunto de $n$ observações por mês $m$ de uma série, denotamos essas observações por $\mathbf{D}_{m}=\left(Z_{t(1, m)}, \ldots, Z_{t(n, m)}\right)^{\prime}$ e denotamos por $\mathbf{Z}_{p_{m}}=\left\{Z_{t(r, m)}\right.$, $\left.t(r, m) \leq p_{m}\right\}$ as $p_{m}$-primeiras observações do processo e por $Z_{n-p_{m}}=\left\{Z_{t(r, m)}\right.$, $\left.t(r, m) \geq p_{m}+1\right\}$ o restante das observações. Podemos usar o modelo (2.3) para calcular os ruídos $a_{t(r, m)}$ por:

$$
a_{t(r, m)}=Z_{t(r, m)}-\sum_{i=1}^{p_{m}} \phi_{i, m} Z_{t(r, m)-i}, t(r, m) \geq p_{m}+1
$$

Note que se $r=1$, a equação (2.33) nos permite calcular $a_{t(1, m)}$ para $m \geq$ $p_{m}+1, \ldots, s$, portanto $a_{t(r, m)}$ para $m=1, \ldots, p_{m}$ só pode ser calculado usando (2.33) para $r=2, \ldots, n$. Então, temos que a função de densidade de probabilidade conjunta dos $a_{t(r, m)}$ é dada por:

$$
\begin{aligned}
f_{m}\left(a_{t(2, m)} \ldots a_{t(n, m)} \mid \phi_{m}, \tau_{m}\right) & =\prod_{r=2}^{n} \tau_{m} \exp \left\{-\frac{\tau_{m}}{2} a_{t(r, m)}^{2}\right\}, \stackrel{\gamma}{m=1, \ldots, p_{m}} \\
f_{m}\left(a_{t(1, m)} \ldots a_{t(n, m)} \mid \phi_{m}, \tau_{m}\right) & =\prod_{r=1}^{n} \tau_{m} \exp \left\{-\frac{\tau_{m}}{2} a_{t(r, m)}^{2}\right\}, m=p_{m}+1, \ldots, s
\end{aligned}
$$

A equação (2.33) relaciona os ruídos $a_{t(r, m)}$ com as observações $\mathbf{Z}_{m}$. Levando em consideração que a relação (2.33) tem jacobiano unitário, podemos escrever a densidade de probabilidade conjunta dos dados $\mathbf{Z}_{m}$ condicionada as $p_{m}$-primeiras observações da série, $\mathbf{Z}_{p_{m}}$, como: 
$f_{m}\left(\mathbf{Z}_{m} \mid \phi_{m}, \tau_{m}\right) \propto\left\{\begin{array}{c}\prod_{r=2}^{n} \tau_{m} \exp \left\{-\frac{\tau_{m}}{2}\left(Z_{t(r, m)}-\sum_{i=1}^{p_{m}} \phi_{i, m} Z_{t(r, m)-i}\right)^{2}\right\}, m=1, \ldots, p_{m} \\ \prod_{r=1}^{n} \tau_{m} \exp \left\{-\frac{\tau_{m}}{2}\left(Z_{t(r, m)}-\sum_{i=1}^{p_{m}} \phi_{i, m} Z_{t(r, m)-i}\right)^{2}\right\}, m=p_{m}+1, \ldots, s\end{array}\right.$

Então, usando a função de densidade de (2.35), condicionando nas $p_{m}$-primeiras observações da série, com $p_{m}<n$, a função de verossimilhança condicional pode ser aproximada por,

$$
L_{m}\left(\phi_{m}, \tau_{m} \mid \mathbf{Z}_{m}\right) \propto r_{m}^{n_{m} / 2} \exp \left\{-\frac{\tau_{m}}{2}\left(\mathbf{Z}_{m}-\mathbf{X}_{m} \boldsymbol{\phi}_{m}\right)^{\prime}\left(\mathbf{Z}_{m}-\mathbf{X}_{m} \phi_{m}\right)\right\}
$$

onde $n_{m}=\left\{\begin{array}{l}n-1, m=1, \ldots, p_{m} \\ n, m=p_{m}+1, \ldots, s\end{array}\right.$.

Em (2.36), para $m \geq p_{m}+1$ definimos:

$$
\begin{aligned}
\mathbf{Z}_{m}= & \left(\begin{array}{c}
\mathbf{Z}_{t(1, m)} \\
\mathbf{Z}_{t(2, m)} \\
\vdots \\
\mathbf{Z}_{t(n, m)}
\end{array}\right)_{n \times 1} ; \mathbf{X}_{m}=\left(\begin{array}{lll}
Z_{t\left(1, \frac{1}{m}\right)-1} & \ldots & Z_{t(1, m)-p_{m}} \\
Z_{t\left(2, \frac{1}{m}\right)-1} & \cdots & Z_{t\left(2, \frac{1}{m}\right)-p_{m}} \\
\vdots & \ddots & \vdots \\
Z_{t\left(n, \frac{1}{m}\right)-1} & \cdots & Z_{t\left(n, \frac{1}{m}\right)-p_{m}}
\end{array}\right)_{n \times p_{m}} \mathbf{e} \\
\phi_{m}= & \left(\begin{array}{c}
\phi_{1, m} \\
\phi_{2, m} \\
\vdots \\
\phi_{p_{m}, m}
\end{array}\right)_{p_{m} \times 1}
\end{aligned}
$$

Quando $m \leq p_{m}$ temos que $\mathbf{Z}_{m}$ é um vetor $(n-1) \times 1$ e $\mathbf{X}_{m}$ é uma matriz $(n-1) \times p_{m}$ identicos aos anteriores porém sem a primeira linha. 
Como estamos trabalhando com modelo periódico é necessário considerarmos a função de verossimilhança como o produto das funções de cada mês como segue

$$
L\left(\phi_{1}, \ldots, \phi_{12}, \tau_{1}, \ldots \tau_{12} \mid \mathbf{Z}_{m}\right) \propto \prod_{m=1}^{12} L_{m}\left(\phi_{m}, \tau_{m} \mid \mathbf{Z}_{m}\right)
$$

onde o vetor de parâmetros associado aos parâmetros $\phi^{\prime}$ s para cada mês denotamos por $\phi_{1}=\left(\phi_{1,1}, \ldots, \phi_{p_{1}, 12}\right)^{\prime}, \ldots, \phi_{12}=\left(\phi_{12,1}, \ldots, \phi_{p_{12}, 12}\right)^{\prime}$

Desta forma, a função de verossimilhança aproximada para todos os meses é dada por

$L\left(\phi_{1}, \ldots, \phi_{12}, \tau_{1}, \ldots \tau_{12} \mid \mathbf{Z}_{m}\right) \propto \prod_{m=1}^{12} \tau_{m}^{n_{m} / 2} \exp \left\{-\frac{\tau_{m}}{2}\left(\mathbf{Z}_{m}-\mathbf{X}_{m} \phi_{m}\right)^{\prime}\left(\mathbf{Z}_{m}-\mathbf{X}_{m} \boldsymbol{\phi}_{m}\right)\right\}$

Segundo Pagano (1978), podemos assumir independência entre os parâmetros, o que resulta trabalharmos com uma função de verossimilhança para cada mês separadamente. Então os cálculos são feitos considerando a função descrita em $(2.36)$.

\subsubsection{Estimadores de Máxima Verossimilhança}

Os estimadores de máxima verossimilhança, EMV, de $\phi_{m}$ e $\tau_{m}$ são os valores que maximizam $L_{m}$ definido anteriormente em (2.36) ou $l_{m}=\log L_{m}$.

O logaritmo da função de verossimilhança aproximada é dado por,

$$
l_{m}\left(\phi_{m}, \tau_{m} \mid \mathbf{Z}_{m}\right) \propto \frac{n_{m}}{2} \ln \tau_{m}-\frac{\tau_{m}}{2}\left(\mathbf{Z}_{m}-\mathbf{X}_{m} \phi_{m}\right)^{\prime}\left(\mathbf{Z}_{m}-\mathbf{X}_{m} \phi_{m}\right)
$$


24

Podemos encontrar os estimadores de máxima verossimilhança $\left(\widehat{\phi}_{m}, \widehat{\tau}_{m}\right)$ resolvendose o sistema de equaçōes,

$$
\begin{gathered}
\frac{\partial l_{m}\left(\phi_{m}, \tau_{m} \mid \mathbf{Z}_{m}\right)}{\partial \phi_{m}}=-\frac{\tau_{m}}{2} X_{m}^{\prime}\left(\mathbf{Z}_{m}-\mathbf{X}_{m} \boldsymbol{\phi}_{m}\right)=0 \\
\frac{\partial l_{m}\left(\phi_{m}, \tau_{m} \mid \mathbf{Z}_{m}\right)}{\partial \tau_{m}}=\frac{n_{m}}{2 \tau_{m}}-\frac{1}{2}\left(\mathbf{Z}_{m}-\mathbf{X}_{m} \phi_{m}\right)^{\prime}\left(\mathbf{Z}_{m}-\mathbf{X}_{m} \phi_{m}\right)=0
\end{gathered}
$$

Assim, $\widehat{\phi}_{m}$ e $\widehat{\tau}_{m}$ são obtidos por,

$$
\widehat{\phi}_{m}=\left(\mathbf{X}_{m}^{\prime} \mathbf{X}_{m}\right)^{-1} \mathbf{X}_{m}^{\prime} \mathbf{Z}_{m}
$$

$$
\widehat{\tau}_{m}^{-1}=\frac{1}{n_{m}}\left(\mathbf{Z}_{m}-\mathbf{X}_{m} \widehat{\phi}_{m}\right)^{\prime}\left(\mathbf{Z}_{m}-\mathbf{X}_{m} \widehat{\phi}_{m}\right)
$$

verificamos que

$$
\begin{aligned}
\left(\mathbf{Z}_{m}-\mathbf{X}_{m} \boldsymbol{\phi}_{m}\right)^{\prime}\left(\mathbf{Z}_{m}-\mathbf{X}_{m} \boldsymbol{\phi}_{m}\right)= & \left(\mathbf{Z}_{m}-\widehat{\mathbf{Z}}_{m}\right)^{\prime}\left(\mathbf{Z}_{m}-\widehat{\mathbf{Z}}_{m}\right)+ \\
& +\left(\phi_{m}-\widehat{\phi}_{m}\right)^{\prime} \mathbf{X}_{m}^{\prime} \mathbf{X}_{m}\left(\boldsymbol{\phi}_{m}-\widehat{\boldsymbol{\phi}}_{m}\right)
\end{aligned}
$$

e definimos

$$
\widehat{\mathbf{Z}}_{m}=\mathbf{X}_{m} \widehat{\phi}_{m}
$$

Substituindo (2.42) em (2.36), a função de verossimilhança aproximada pode ser reescrita como,

$$
\begin{aligned}
L_{m}\left(\boldsymbol{\phi}_{m}, \boldsymbol{\tau}_{m} \mid \mathbf{Z}_{m}\right) \propto & \tau_{m}^{n_{m} / 2} \exp \left\{-\frac{\tau_{m}}{2}\left[\left(\boldsymbol{\phi}_{m}-\widehat{\boldsymbol{\phi}}_{m}\right)^{\prime} \mathbf{X}_{m}^{\prime} \mathbf{X}_{m}\left(\boldsymbol{\phi}_{m}-\widehat{\boldsymbol{\phi}}_{m}\right)+\right.\right. \\
& \left.\left.+\left(\mathbf{Z}_{m}-\widehat{\mathbf{Z}}_{m}\right)^{\prime}\left(\mathbf{Z}_{m}-\widehat{\mathbf{Z}}_{m}\right)\right]\right\}
\end{aligned}
$$


A função de verossimilhança (2.43) é chamada de função de verossimilhança aproximada por ter sido construída a partir da densidade conjunta dos ruídos condicionada as $p_{m}$-primeiras observações $\mathbf{Z}_{p_{m}}$. Esta função representa somente a densidade de probabilidade conjunta das observações $\mathbf{Z}_{m}$, isto é , $L_{m}\left(\phi_{m}, \boldsymbol{\tau}_{m} \mid \mathbf{Z}_{m}\right)$ é a função de verossimilhança para um determinado mês $m$ condicionada somente a parte dos dados dada por $\mathbf{Z}_{m}$.

\subsubsection{Inferência sobre os parâmetros $\phi_{m}$ e $\tau_{m}$}

A partir dos estimadores de máxima verossimilhança para $\phi_{m}$ e $\tau_{m}$, podemos obter intervalos de confiança e testes de hipóteses para os parâmetros do modelo para cada mês $m$, usando a teoria assintótica para grandes amostras, baseadas na matriz de informação de Fisher ou na matriz de informação obdervada. Assim, considerando o resultado,

$$
\left(\widehat{\boldsymbol{\phi}}_{m}, \widehat{\tau}_{m}\right) \stackrel{a}{\sim} N\left[\left(\phi_{m}, \tau_{m}\right), I^{-1}\left(\widehat{\boldsymbol{\phi}}_{m}, \widehat{\tau}_{m}\right)\right]
$$

A matriz de informação observada é definida por,

$$
I^{-1}\left(\widehat{\phi}_{m}, \widehat{\tau}_{m}\right)=\left[\begin{array}{cc}
-\frac{\partial^{2} l_{m}\left(\widehat{\phi}_{m}, \widehat{\tau}_{m}\right)}{\partial^{2} \phi_{m}} & -\frac{\partial^{2} l_{m}\left(\widehat{\phi}_{m}, \widehat{\tau}_{m}\right)}{\partial \phi_{m} \partial \tau_{m}} \\
-\frac{\partial^{2} l_{m}\left(\widehat{\phi}_{m}, \widehat{\tau}_{m}\right)}{\partial \phi_{m} \partial \tau_{m}} & -\frac{\partial^{2} l_{m}\left(\widehat{\phi}_{m}, \widehat{\tau}_{m}\right)}{\partial^{2} \tau_{m}}
\end{array}\right]
$$

assim,

$$
I^{-1}\left(\widehat{\boldsymbol{\phi}}_{m}, \widehat{\boldsymbol{\tau}}_{m}\right)=\left[\begin{array}{cc}
\boldsymbol{\tau}_{m} \mathbf{X}_{m}^{\prime} \mathbf{X}_{m} & -\mathbf{X}_{m}^{\prime} \mathbf{Z}_{m}+\mathbf{X}_{m}^{\prime} \mathbf{X}_{m} \widehat{\boldsymbol{\phi}}_{m} \\
-\mathbf{X}_{m}^{\prime} \mathbf{Z}_{m}+\mathbf{X}_{m}^{\prime} \mathbf{X}_{m} \widehat{\boldsymbol{\phi}}_{m} & \frac{1}{2} n_{m} \widehat{\tau}_{m}^{2}
\end{array}\right]
$$


e $I^{-1}\left(\widehat{\phi}_{m}, \widehat{\tau}_{m}\right)$, o inverso da matriz de informação observada, dada em (2.46) representa a matriz de variância e covariância assintótica de $\phi_{m}$ e $\tau_{m}$. Portanto,

$$
\begin{gathered}
\operatorname{Var}\left(\widehat{\phi}_{m}\right)=\widehat{\tau}_{m}^{-1}\left(\mathbf{X}_{m}^{\prime} \mathbf{X}_{m}\right)^{-1} \\
\operatorname{Var}\left(\widehat{\tau}_{m}\right)=\frac{2}{n_{m}} \widehat{\tau}_{m}^{2}
\end{gathered}
$$

A partir da normalidade assintótica dos estimadores, podemos construir intervalos de confiança e testes de hipóteses para $\phi_{m}$ e $\tau_{m}$, usando a inversa da matriz de informação observada.

Os intervalos de confiança assintóticos para $\phi_{m}$ e $\tau_{m}$, considerando um nível de significância $(1-\alpha)$ são dados por:

$$
\begin{aligned}
& I C\left(\phi_{m}\right)=\left[\widehat{\phi}_{m} \pm z_{\alpha / 2} \sqrt{\operatorname{Var}\left(\widehat{\phi}_{m}\right)}\right] \\
& I C\left(\tau_{m}\right)=\left[\widehat{\tau}_{m} \pm z_{\alpha / 2} \sqrt{\operatorname{Var}\left(\widehat{\tau}_{m}\right)}\right]
\end{aligned}
$$

onde $z_{\alpha / 2}$ é um percentil da distribuição normal padronizada de uma tabela estatística.

\subsection{Identificação da Ordem do Modelo}

Identificar a ordem de um modelo consiste em determinar o valor de $p_{m}$, ou seja, determinar o número adequado de termos que devemos considerar no modelo PAR. A escolha de um valor pequeno de $p_{m}$, por exemplo, $p_{m}=1$ pode levar a uma representação inadequada da série, por outro lado a escolha de um valor alto de 
$p_{m}$, por exemplo, $p_{m}=6$ pode levar a um modelo com grau de complexidade desnecessário, ou ainda, muitos modelos podem se mostrar igualmente adequados para representar um conjunto de dados. Para solucionar esses problemas usamos o critério de escolha do modelo.

O modelo adequado é aquele com o menor número de parâmetros possível, isto é, o modelo que melhor atenda ao critério de parcimoniosidade de Box [Box, Jenkins e Reinsel-1994]. Um dos critérios propostos na literatura é o Critério de Informação Bayesiano, BIC. O critério BIC é proposto por Schwarz (1978) e futuramente foi estudado por Katz (1981) como uma alternativa ao AIC.

No caso de séries periodicamente estacionárias, o Critério de Informação Bayesiano é fatorado para obtermos um critério separado para cada mês $m$. Então, um critério geral pode ser calculado como segue:

$$
B I C=\sum_{m=1}^{s} B I C_{m} ; B I C_{m} \simeq \ln \left(\widehat{\tau}_{m}^{-1}\right)+r \frac{\ln \left(n_{m}\right)}{n_{m}}
$$

onde $\widehat{\tau}_{m}$ denota o estimador do inverso da variância residual e $r=p_{m}+1$ denota o número de parâmetros estimados no modelo incluindo o termo constante e $n$ denota o tamanho da série observada. 


\section{CAPÍTULO 3}

\section{Análise Bayesiana para Modelos PAR}

Neste capítulo, apresentamos a análise Bayesiana para modelos periódicos de séries temporais, modelos PAR. Estamos interessados em estimar o valor dos parâmetros desse modelo. Na análise Bayesiana os parâmetros do modelo são considerados variáveis aleatórias e expressamos nossa incerteza sobre esses parâmetros através de uma densidade de probabilidade a priori.

Uma vez definido o modelo para os dados e a distribuição a priori, devemos combinar essa informação usando o teorema de Bayes. Este procedimento resulta em uma densidade a posteriori para $\phi_{m}$ e $\tau_{m}$, dada por:

$$
\Pi_{m}\left(\phi_{m}, \boldsymbol{\tau}_{m} \mid \mathbf{Z}_{m}\right)=\frac{L_{m}\left(\boldsymbol{\phi}_{m}, \boldsymbol{\tau}_{m} \mid \mathbf{Z}_{m}\right) \Pi_{m}\left(\boldsymbol{\phi}_{m}, \boldsymbol{\tau}_{m}\right)}{\int_{\phi_{1}} \ldots \int_{\phi_{m}} \int_{\tau_{m}} L_{m}\left(\boldsymbol{\phi}_{m}, \boldsymbol{\tau}_{m} \mid \mathbf{Z}_{m}\right) \Pi_{m}\left(\boldsymbol{\phi}_{m}, \boldsymbol{\tau}_{m}\right) d \phi_{1} \ldots d \phi_{m} d \tau_{m}}
$$

Como o denominador de (3.1) não depende nem de $\phi_{m}$ nem de $\tau_{m}$, o teorema de Bayes pode ser reescrito como:

$$
\Pi_{m}\left(\phi_{m}, \tau_{m} \mid \mathbf{Z}_{m}\right) \propto L_{m}\left(\phi_{m}, \tau_{m} \mid \mathbf{Z}_{m}\right) \Pi_{m}\left(\phi_{m}, \tau_{m}\right)
$$

O processo de inferência baseia-se na distribuição a posteriori de $\phi_{m}$ e $\tau_{m}$. Portanto, essa distribuição pode ser utilizada para a estimativa pontual dos parâmetros de interesse ( por exemplo, a média ou a moda a posteriori de $\phi_{m}$ e $\tau_{m}$ ) e para a construção de intervalos de credibilidade (estimativa Bayesiana por intervalo). 
O método Bayesiano apresenta algumas limitações nas aplicações quando relacionadas à resolução de integrais Bayesianas para obtenção das distribuições marginais. que muitas vezes não apresentam soluções analíticas e exigem o uso de métodos de aproximação de integrais, tal como o método de Laplace [Tierney e Kadane-1986] Uma outra estratégia existente que tem sido muito utilizada recentemente são as técnicas de simulação de Monte Carlo em Cadeias de Markov (MCMC), como amostrador de Gibbs e o algoritmo de Metropolis-Hastings [Chib e Greenberg 1995].

Neste trabalho. estudamos os modelos auto-regressivos periódicos, considerando três alternativas de densidades a priori para os parâmetros desse modelo. Apesar de conseguirmos calcular analiticamente em dois casos as densidades a posteriori marginais também utilizamos os métodos de simulação por serem precisos e de fácil implementação computacional, principalmente para modelos com muitos parâmetros como é o caso dos modelos PAR.

A convergência (em distribuição) dos algoritmos amostrador de Gibbs e Metropolis-Hastings pode ser verificada informalmente através de métodos gráficos, quantitativos ou qualitativos e também pode ser verificada formalmente através de vários métodos propostos na litertura. Utilizamos para a monitoração de convergência o critério proposto por Gelman e Rubin (1992). 


\subsection{Densidade a Priori Não-Informativa}

Antes de analisar os dados podemos incorporar conhecimento prévio a respeito dos parâmetros do modelo. Uma forma de se fazer isto é em termos de uma densidade de probabilidade para os parâmetros. Essa densidade de probabilidade é identificada como densidade a priori. Quando se tem pouca informação a respeito dos parâmetros, o grau de não conhecimento é representado através da densidade a priori não-informativa Para isso, existem várias regras para se formular uma priori não-informativa, aqui usamos a priori não-informativa de Jeffreys [Box e Tiao-1973].

Considerando o modelo $\operatorname{PAR}\left(p_{m}\right)$ dado em (2.3), adotando a densidade a priori de Jeffreys para $\phi_{m}$ e $\tau_{m}$ e assumindo independência entre os parâmetros, encontramos uma densidade a priori conjunta dada por,

$$
\Pi_{m}\left(\phi_{m}, \tau_{m}\right) \propto \frac{1}{\tau_{m}}, \tau_{m}>0
$$

\subsubsection{Densidade a Posteriori Conjunta para $\phi_{m}$ e $\tau_{m}$}

Combinando-se a priori (3.3) e a função de verossimilhança (2.36), obtemos a densidade a posteriori conjunta dada pela expressão:

$$
\begin{gathered}
\sigma^{2} \delta_{m} x^{\prime} X_{m} \\
\Pi_{m}\left(\phi_{m}, \boldsymbol{\tau}_{m} \mid \mathbf{Z}_{m}\right) \propto \tau_{m}^{\frac{n_{m}}{2}-1} \exp \left\{-\frac{\tau_{m}}{2}\left[\left(\phi_{m}-\widehat{\phi}_{m}\right)^{\prime} \mathbf{X}_{m}^{\prime} \mathbf{X}_{m}\left(\phi_{m}-\widehat{\phi}_{m}\right)+\right.\right. \\
\left.\left.+\left(\mathbf{Z}_{m}-\widehat{\mathbf{Z}}_{m}\right)^{\prime}\left(\mathbf{Z}_{m}-\widehat{\mathbf{Z}}_{m}\right)\right]\right\}
\end{gathered}
$$

onde $\Pi_{m}\left(\phi_{m}, \tau_{m} \mid \mathbf{Z}_{m}\right)$ tem uma distribuição Normal-Gama. 
Em análise bayesiana, inferências são tipicamente baseadas nas densidades a posteriori marginais para os parâmetros envolvidos. Portanto, com a posteriori conjunta podemos encontrar as densidades a posteriori marginais para $\phi_{m}$ e $\tau_{m}$, como mostramos a seguir.

\subsubsection{Densidade a Posteriori Marginal para $\tau_{m}$}

Neste caso, onde estamos usando uma priori não-informativa, a densidade a posteriori é uma função conhecida, então podemos encontrar a densidade a posteriori marginal para $\tau_{m}$ integrando diretamente (3.4) com relação aos parâmetros $\phi_{m}$. Procedendo desta forma, temos:

$$
\begin{aligned}
\Pi_{m}\left(\tau_{m} \mid \mathbf{Z}_{m}\right) \propto & \tau_{m}^{\frac{n_{m}}{2}-1} \exp \left\{-\frac{\tau_{m}}{2}\left(\mathbf{Z}_{m}-\widehat{\mathbf{Z}}_{m}\right)^{\prime}\left(\mathbf{Z}_{m}-\widehat{\mathbf{Z}}_{m}\right)\right\} \times \\
& \times \int_{\phi_{m}} \exp \left\{\frac{-\tau_{m}}{2}\left(\phi_{m}-\widehat{\phi}_{m}\right)^{\prime} \mathbf{X}_{m}^{\prime} \mathbf{X}_{m}\left(\phi_{m}-\widehat{\phi}_{m}\right)\right\} d \phi_{m}
\end{aligned}
$$

A integral de (3.5), representa uma integral multipla para o vetor de parâmetros $\phi_{m}$, então resolvendo essa integral temos,

$$
\Pi_{m}\left(\tau_{m} \mid \mathbf{Z}_{m}\right) \propto \tau_{m}^{\frac{n_{m}-p_{m}}{2}}-1 \exp \left\{-\frac{\tau_{m}}{2}\left(\mathbf{Z}_{m}-\widehat{\mathbf{Z}}_{m}\right)^{\prime}\left(\mathbf{Z}_{m}-\widehat{\mathbf{Z}}_{m}\right)\right\}
$$

Então, temos que $\tau_{m}$ tem uma densidade a posteriori $\operatorname{Gama}\left(\frac{n_{m}-p_{m}}{2}, B_{m}\right)$, onde $B_{m}=\left(\mathbf{Z}_{m}-\widehat{\mathbf{Z}}_{m}\right)^{\prime}\left(\mathbf{Z}_{m}-\widehat{\mathbf{Z}}_{m}\right) / 2$. Os estimadores bayesianos de $\oint_{m}$ e $\tau_{m}$ são dados por:

$$
E\left(\boldsymbol{\tau}_{m} \mid \mathbf{Z}_{m}\right)=n_{m}-p_{m}\left[\left(\mathbf{Z}_{m}-\widehat{\mathbf{Z}}_{m}\right)^{\prime}\left(\mathbf{Z}_{m}-\widehat{\mathbf{Z}}_{m}\right)\right]^{-1}
$$




$$
\operatorname{Var}\left(\boldsymbol{\tau}_{m} \mid \mathbf{Z}_{m}\right)=2\left(n_{m}-p_{m}\right)\left[\left(\mathbf{Z}_{m}-\widehat{\mathbf{Z}}_{m}\right)^{\prime}\left(\mathbf{Z}_{m}-\widehat{\mathbf{Z}}_{m}\right)\right]^{-2}
$$

\subsubsection{Densidade a Posteriori Marginal para $\phi_{m}$}

Da mesma forma, integrando (3.4) com relação a $\tau_{m}$, temos a densidade a posteriori marginal para os parâmetros $\phi_{m}$, dada por:

$$
\begin{aligned}
& \Pi_{m}\left(\phi_{m} \mid \mathbf{Z}_{m}\right) \propto \int_{\tau_{m}} \tau_{m}^{\frac{n_{m}-\gamma_{m}}{2}}{ }^{-1} \exp \left\{-\frac{\boldsymbol{\tau}_{m}}{2}\left[\left(\phi_{m}-\widehat{\phi}_{m}\right)^{\prime} \mathbf{X}_{m}^{\prime} \mathbf{X}_{m}\left(\phi_{m}-\widehat{\phi}_{m}\right)+\right.\right. \\
& \left.\left.+\left(\mathbf{Z}_{m}-\widehat{\mathbf{Z}}_{m}\right)^{\prime}\left(\mathbf{Z}_{m}-\widehat{\mathbf{Z}}_{m}\right)\right]\right\} d \tau_{m}
\end{aligned}
$$

assim, solucionando essa integral temos,

$$
\Pi_{m}\left(\phi_{m} \mid \mathbf{Z}_{m}\right) \propto\left[\left(\phi_{m}-\widehat{\phi}_{m}\right)^{\prime} \mathbf{X}_{m}^{\prime} \mathbf{X}_{m}\left(\phi_{m}-\widehat{\phi}_{m}\right)+\left(\mathbf{Z}_{m}-\widehat{\mathbf{Z}}_{m}\right)^{\prime}\left(\mathbf{Z}_{m}-\widehat{\mathbf{Z}}_{m}\right)\right]^{-\frac{n_{m}-\hat{\alpha}}{2}}
$$

Após algumas simplificações algébricas, podemos reescrever a densidade a posteriori dos parâmetros $\phi_{m}$, como:

$$
\Pi_{m}\left(\phi_{m}\right) \propto\left[1+\frac{\left(\phi_{m}-\widehat{\phi}_{m}\right)^{\prime} V_{m}\left(\phi_{m}-\widehat{\phi}_{m}\right)}{v_{m}}\right]^{-\frac{v_{m}+p_{m}}{2}}
$$

onde,

$$
\begin{aligned}
\widehat{\phi}_{m} & =\left(\mathbf{X}_{m}^{\prime} \mathbf{X}_{m}\right)^{-1} \mathbf{X}_{m}^{\prime} \mathbf{Z}_{m} \\
V_{m} & =\left(n_{m}-p_{m}\right) \mathbf{X}_{m}^{\prime} \mathbf{X}_{m}\left[\left(\mathbf{Z}_{m}-\widehat{\mathbf{Z}}_{m}\right)^{\prime}\left(\mathbf{Z}_{m}-\widehat{\mathbf{Z}}_{m}\right)\right]^{-1} \\
v_{m} & =\left(n_{m}-p_{m}\right)
\end{aligned}
$$

Portanto, $\phi_{m}$ tem densidade a posteriori marginal $t$-Student $p_{m}$-dimensional, com $n_{m}-p_{m}$ graus de liberdade, vetor de localização e matriz de covariância dada 
por,

$$
\begin{aligned}
E\left(\phi_{m} \mid \mathbf{Z}_{m}\right) & =\widehat{\phi}_{m} \\
\operatorname{Var}\left(\phi_{m} \mid \mathbf{Z}_{m}\right) & =\frac{v_{m}}{v_{m}-2} V_{m}^{-1}=\frac{\left(\mathbf{Z}_{m}-\widehat{\mathbf{Z}}_{m}\right)^{\prime}\left(\mathbf{Z}_{m}-\widehat{\mathbf{Z}}_{m}\right)\left(\mathbf{X}_{m}^{\prime} \mathbf{X}_{m}\right)^{-1}}{n_{m}-p_{m}-2}
\end{aligned}
$$

Notamos que com o uso da densidade a priori não-informativa o estimador Bayesiano $E\left(\phi_{m} \mid \mathbf{Z}_{m}\right)$ coincide com estimador de máxima verossimilhança $\widehat{\phi}_{m}$.

\subsubsection{Densidades a Posteriori Condicionais para $\tau_{m}$ e $\phi_{m}$}

A densidade condicional de $\boldsymbol{\tau}_{m}$ para o algoritmo amostrador de Gibbs é obtida através da densidade a posteriori conjunta de $\phi_{m}$ e $\tau_{m}(3.4)$, considerando $\tau_{m}$ condicionado aos dados, $\mathbf{Z}_{m}$ e aos parâmetros $\phi_{m}$, temos,

$$
\Pi_{m}\left(\boldsymbol{\tau}_{m} \mid \phi_{m}, \mathbf{Z}_{m}\right) \sim \Gamma\left(\frac{n_{m}}{2}, B_{m}\right)
$$

onde $B_{m}=\frac{1}{2}\left[\left(\phi_{m}-\widehat{\phi}_{m}\right)^{\prime} \mathbf{X}_{m}^{\prime} \mathbf{X}_{m}\left(\phi_{m}-\widehat{\phi}_{m}\right)+\left(\mathbf{Z}_{m}-\widehat{\mathbf{Z}}_{m}\right)^{\prime}\left(\mathbf{Z}_{m}-\widehat{\mathbf{Z}}_{m}\right)\right]$.

A densidade condicional de $\tau_{m}(3.8)$ tem forma padronizada Gama.

A densidade condicional de $\phi_{m}$ é obtida através da densidade a posteriori conjunta (3.4), considerando $\phi_{m}$ condicionado aos dados, $\mathbf{Z}_{m}$ e aos parâmetros $\tau_{m}$, temos,

$$
\Pi_{m}\left(\phi_{m} \mid \tau_{m}, \mathbf{Z}_{m}\right) \sim N\left(\widehat{\phi}_{m},\left(\tau_{m} V_{m}\right)^{-1}\right)
$$

onde, 


$$
\begin{aligned}
\hat{\boldsymbol{\phi}}_{m} & =\left(\mathbf{X}_{m}^{\prime} \mathbf{X}_{m}\right)^{-1} \mathbf{X}_{m}^{\prime} \mathbf{Z}_{m} \\
V_{m} & =\left(\mathbf{X}_{m}^{\prime} \mathbf{X}_{m}\right)^{\natural 1}
\end{aligned}
$$

Observamos que a densidade condicional de $\phi_{m}$ (3.9) tem forma padronizada Normal Multivariada. O algoritmo amostrador de Gibbs pode ser usado para gerar amostras de $\tau_{m}$ e $\phi_{m}$.

\subsection{Densidades a Priori Informativas}

Para representarmos o grau de conhecimento sobre os parâmetros do modelo, vamos considerar duas densidades a priori informativas. Inicialmente consideramos uma priori conjugada Normal-Gama e outra opção é considerar uma priori $t$ Student para $\phi_{m}$ e uma priori Gama para $\tau_{m}$.

\subsection{Densidade a Priori Normal-Gama}

Analisando a forma da função de verossimilhança (2.36), sugerimos uma densidade a priori conjugada Normal-Gama para $\phi_{m}$ e $\tau_{m}$, dada por,

$$
\Pi_{m}\left(\phi_{m}, \tau_{m}\right)=\tau_{m}^{\left(p_{m}+2 \alpha_{m} / 2\right)-1} \exp \left\{-\frac{\tau_{m}}{2}\left[2 \beta_{m}+\left(\phi_{m}-\mu_{m}\right)^{\prime} P_{m}\left(\phi_{m}-\mu_{m}\right)\right\}\right.
$$

onde,

$$
\begin{aligned}
& \Pi_{1}\left(\phi_{m} \mid \tau_{m}\right) \propto \tau_{m}^{\gamma_{m}^{\beta_{m}} / 2} \exp \left\{-\frac{\tau_{m}}{2}\left[\left(\phi_{m}-\mu_{m}\right)^{\prime} P_{m}\left(\phi_{m}-\mu_{m}\right)\right\}\right. \\
& \frac{\rho m}{2} \frac{z+m}{\gamma}-1 \cdot \frac{x}{\gamma}
\end{aligned}
$$




$$
\Pi_{2}\left(\tau_{m}\right) \propto \tau_{m}^{\alpha_{m}-1} \exp \left\{-\beta_{m} \tau_{m}\right\}
$$

Sendo $\Pi_{1}\left(\phi_{m} \mid \tau_{m}\right)$ uma distribuição Normal com parâmetros $\mu_{m} \in \Re^{p_{m}}$ e matriz de precisão $\tau_{m}^{-1} P_{m}^{-1}, P_{m} \in \Re^{p_{m} \times p_{m}}$ e $\Pi_{2}\left(\tau_{m}\right)$ uma distribuição $\operatorname{Gama}\left(\alpha_{m}, \beta_{m}\right)$.

Os parâmetros $\mu_{m}, \alpha_{m}, \beta_{m}$ e $P_{m}$ que auxiliam na especificação da priori são chamados hiperparâmetros e são supostamente conhecidos.

\subsubsection{Densidade a Posteriori Conjunta para $\phi_{m}$ e $\tau_{m}$}

Combinando a informação a priori (3.10) com a função de verossimilhança (2.36), obtemos a densidade a posteriori da seguinte forma,

onde,

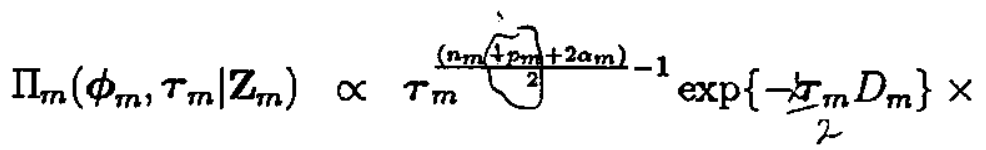

$$
\begin{aligned}
& \times \tau_{m}^{p_{m} / 2} \exp \left\{-\frac{\tau_{m}}{2}\left(\phi_{m}-\widehat{\phi}_{m}\right)^{\prime} V_{m}\left(\phi_{m}-\widehat{\phi}_{m}\right)\right\} \\
& \frac{p r}{2}+\frac{2 d r}{2}-1 \quad \frac{r}{2}
\end{aligned}
$$

$$
\begin{aligned}
\hat{\phi}_{m}= & \left(\mathbf{X}_{m}^{\prime} \mathbf{X}_{m}+P_{m}\right)^{-1}\left(\mathbf{X}_{m}^{\prime} \mathbf{Z}_{m}+P_{m} \mu_{m}\right) \\
V_{m}= & \mathbf{X}_{m}^{\prime} \mathbf{X}_{m}+P_{m}- \\
D_{m}= & \beta_{m}+\frac{1}{2}\left[\left(\mathbf{Z}_{m}^{\prime} \mathbf{Z}_{m}+\mu_{m}^{\prime} P_{m} \mu_{m}\right)-\right. \\
& \left.\left(X_{m}^{\prime} \mathbf{Z}_{m}+P_{m} \mu_{m}\right)^{\prime}\left(X_{m}^{\prime} X_{m}+P_{m}\right)^{-1}\left(X_{m}^{\prime} \mathbf{Z}_{m}+P_{m} \mu_{m}\right)\right]
\end{aligned}
$$

Devido a conjugação, temos que a densidade a posteriori conjunta (3.11) tem uma distribuição Normal-Gama. Assim, podemos determinar analiticamente as densidades a posteriori marginais para os parâmetros de interesse. 


\subsubsection{Densidade a Posteriori Marginal para $\tau_{m}$}

A partir da densidade a posteriori conjunta para os parâmetros $\phi_{m}$ e $\tau_{m}$ podemos encontrar a densidade a posteriori marginal para $\tau_{m}$, integrando (3.11) com relação aos parâmetros $\phi_{m}$. Procedendo desta forma, temos,

$$
\begin{aligned}
\Pi_{m}\left(\tau_{m} \mid \mathbf{Z}_{m}\right)= & \tau_{m}^{\frac{\left(n_{m}=p_{m}+2 a_{m}\right)}{2}-1} \exp \left\{-\tau_{m} D_{m}\right\} \times \\
& \times \int_{\phi_{m}} \tau_{m}^{p_{m} / 2} \exp \left\{-\frac{\tau_{m}}{2}\left(\phi_{m}-\widehat{\phi}_{m}\right)^{\prime} V_{m}\left(\phi_{m}-\widehat{\phi}_{m}\right)\right\} d \phi_{m}
\end{aligned}
$$

assim,

$$
\Pi_{m}\left(\tau_{m} \mid \mathbf{Z}_{m}\right) \propto \tau_{m}^{\frac{\left(n_{m}-p_{m}+2 \alpha_{m}\right)}{2}-1} \exp \left\{-\tau_{m} D_{m}\right\}
$$

O valor esperado e a variância a posteriori de $\tau_{m}$, são dados por,

$$
\begin{gathered}
E\left(\boldsymbol{\tau}_{m} \mid \mathbf{Z}_{m}\right)=\left(n_{m}-p_{m}+2 \alpha_{m}\right)\left(2 D_{m}\right)^{-1} \\
\operatorname{Var}\left(\boldsymbol{\tau}_{m} \mid \mathbf{Z}_{m}\right)=E\left(\boldsymbol{\tau}_{m} \mid \mathbf{Z}_{m}\right)\left(D_{m}\right)^{-1}
\end{gathered}
$$

\subsubsection{Densidade a Posteriori Marginal para $\phi_{m}$}

Da mesma forma, integrando (3.11) com relação a $\boldsymbol{\tau}_{m}$, temos a densidade a posteriori marginal para os parâmetros $\phi_{m}$, dada por,

$$
\Pi_{m}\left(\phi_{m} \mid \mathbf{Z}_{m}\right)=\int_{\tau_{m}} \boldsymbol{\tau}_{m}^{\frac{\left(n_{m}+2 \alpha_{m}\right)}{2}-1} \exp \left\{\frac{-\tau_{m}}{2}\left(\phi_{m}-\widehat{\phi}_{m}\right)^{\prime} V_{m}\left(\phi_{m}-\widehat{\phi}_{m}\right)+2 D_{m}\right\} d \tau_{m}
$$


resolvendo a integral, temos.

$$
\Pi_{m}\left(\phi_{m} \mid \mathbf{Z}_{m}\right) \propto\left[\left(\phi_{m}-\widehat{\phi}_{m}\right)^{\prime} V_{m}\left(\phi_{m}-\widehat{\phi}_{m}\right)+2 D_{m}\right]^{\frac{\left(\pi_{m}+2 \alpha_{m}\right)}{2}}
$$

reagrupando os termos, podemos escrever (3.15), como:

$$
\Pi_{m}\left(\phi_{m}\right) \propto\left[1+\frac{\left(\phi_{m}-\widehat{\phi}_{m}\right)^{\prime} W_{m}\left(\phi_{m}-\widehat{\phi}_{m}\right)}{n-p_{m}+2 \alpha_{m}}\right]^{-\frac{\left(n_{m}+2 \alpha_{m}\right)}{2}}
$$

onde $W_{m}=\left(n_{m}-p_{m}+2 \alpha_{m}\right) V_{m}\left(2 D_{m}\right)^{-1}$.

Portanto, vemos que $\phi_{m}$ tem densidade a posteriori marginal $t$-Student $p_{m}$ dimensional com $v_{m}=\left(n_{m}-p_{m}+2 \alpha_{m}\right)$ graus de liberdade, vetor de localização $\widehat{\phi}_{m}$ e a matriz de precisão $W_{m}$. Então, podemos estimar os parâmetros $\phi_{m}$, pelo valor esperado da densidade (3.16) e a matriz de covariância dos parâmetros pode ser obtida diretamente da matriz de precisão $W_{m}$.

Assim, temos:

$$
\begin{gathered}
E\left(\phi_{m} \mid \mathbf{Z}_{m}\right)=\left(X_{m}^{\prime} X_{m}+P_{m}\right)^{-1}\left(X_{m}^{\prime} \mathbf{Z}_{m}+P_{m} \mu_{m}\right) \\
\operatorname{Var}\left(\phi_{m} \mid \mathbf{Z}_{m}\right)=\frac{v_{m}}{v_{m}-2} W_{m}^{-1}=\frac{\left(2 D_{m}\right)\left(X_{m}^{\prime} X_{m}+P_{m}\right)^{-1}}{\left(n_{m}-p_{m}+2 \alpha_{m}-2\right)}
\end{gathered}
$$

Podemos notar de (3.17) que o valor esperado a posteriori, $E\left(\phi_{m} \mid \mathbf{Z}_{m}\right)$ é uma média ponderada do estimador de máxima verossimilhança $\widehat{\phi}_{m}$ e da média da densidade a priori, $\mu_{m}$, ou seja:

$$
E\left(\phi_{m} \mid \mathbf{Z}_{m}\right)=\left(X_{m}^{\prime} X_{m}+P_{m}\right)^{-1}\left[\left(X_{m}^{\prime} X_{m}\right) \widehat{\phi}_{m}+P_{m} \mu_{m}\right]
$$




\subsubsection{Densidades a Posteriori Condicionais para $\tau_{m}$ e $\phi_{m}$}

A densidade de $\tau_{m}$, condicionada aos dados, $\mathbf{Z}_{m}$ e aos parâmetros $\phi_{m}$, é dada por,

$$
\Pi_{m}\left(\tau_{m} \mid \phi_{m}, \mathbf{Z}_{m}\right) \sim \Gamma\left(\frac{\left(n_{m}+2 \alpha_{m}\right)}{2}, D_{m}+\frac{1}{2}\left(\phi_{m}-\widehat{\phi}_{m}\right)^{\prime} V_{m}\left(\phi_{m}-\widehat{\phi}_{m}\right)\right)
$$

onde,

$$
\begin{aligned}
D_{m} & =\beta_{m}+\frac{1}{2}\left[\left(\mathbf{Z}_{m}^{\prime} \mathbf{Z}_{m}+\mu_{m}^{\prime} P_{m} \mu_{m}\right)-\left(X_{m}^{\prime} \mathbf{Z}_{m}+P_{m} \mu_{m}\right)^{\prime}\left(X_{m}^{\prime} X_{m}+P_{m}\right)^{-1}\left(X_{m}^{\prime} \mathbf{Z}_{m}+P_{m} \mu_{n}\right.\right. \\
V_{m} & =\mathbf{X}_{m}^{\prime} \mathbf{X}_{m}+P_{m}
\end{aligned}
$$

A densidade condicional de $\tau_{m}$ (3.20) tem forma padronizada Gama, então utilizamos o algoritmo amostrador Gibbs.

A densidade de $\phi_{m}$ condicionado aos parâmetros $\tau_{m}$ e aos dados, $\mathbf{Z}_{m}$ é dada por,

$$
\Pi_{m}\left(\phi_{m} \mid \boldsymbol{\tau}_{m}, \mathbf{Z}_{m}\right) \sim N\left(\widehat{\phi}_{m},\left(\boldsymbol{\tau}_{m} V_{m}\right)^{-1}\right)
$$

onde

$$
\begin{aligned}
\hat{\phi}_{m} & =\left(X_{m}^{\prime} X_{m}+P_{m}\right)^{-1}\left(X_{m}^{\prime} \mathbf{Z}_{m}+P_{m} \mu_{m}\right) \\
V_{m} & =X_{m}^{\prime} X_{m}+P_{m}
\end{aligned}
$$

A densidade condicional de $\phi_{m}$ (3.21) denota a distribuição Normal Multivariada. 


\subsection{Densidade a Priori $t$-Student para $\phi_{m}$ e Priori}

\section{Gama para $\tau_{m}$}

Com o objetivo de evitar uma densidade a priori conjugada para os parâmetros $\phi_{m}$ e $\tau_{m}$, utilizamos a priori $t$ - Student $p_{m^{-}}$dimensional para os parâmetros $\phi_{m}$ e uma densidade a priori Gama para $\tau_{m}$.

Supondo independência para os parâmetros $\phi_{m}$ e $\tau_{m}$, temos:

$$
\Pi\left(\phi_{m}, \tau_{m}\right) \propto \Pi_{1}\left(\phi_{m}\right) \Pi_{2}\left(\tau_{m}\right)
$$

com as respectivas densidades a priori:

$$
\begin{gathered}
\Pi_{1}\left(\phi_{m}\right) \propto\left[1+\frac{\left(\phi_{m}-\mu_{m}\right)^{\prime} P_{m}\left(\phi_{m}-\mu_{m}\right)}{k_{m}}\right]^{-\frac{\left(k_{m}+p_{m}\right)}{2}} \\
\Pi_{2}\left(\tau_{m}\right) \propto \tau_{m}^{\alpha_{m}-1} \exp \left\{-\beta_{m} \tau_{m}\right\}
\end{gathered}
$$

onde $k_{m}$ é o grau de liberdade da priori $t$-Student, $\mu_{m}$ é o vetor de localização e $P_{m}$ é a matriz de precisão e $\alpha_{m}$ e $\beta_{m}$ são os hiperparâmetros da Gama.

\subsubsection{Densidade a Posteriori Conjunta para $\phi_{m}$ e $\tau_{m}$}

Considerando as densidades a priori (3.22) e (3.23) e a função de verossimilhança (2.36), a densidade a posteriori conjunta para $\phi_{m}$ e $\tau_{m}$ é dada por:

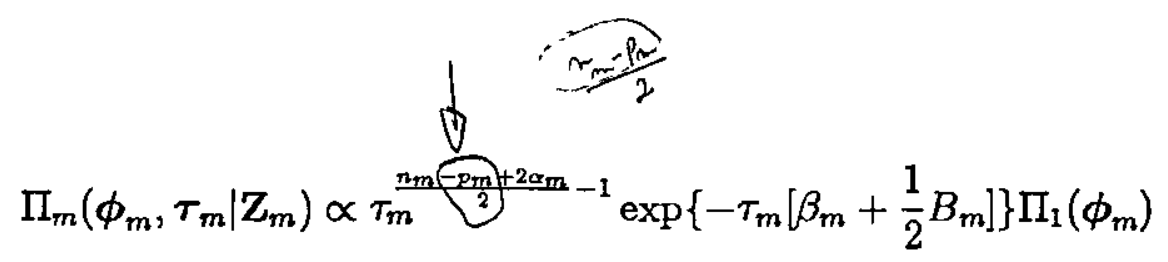

onde $B_{m}=\left(\phi_{m}-\widehat{\phi}_{m}\right)^{\prime} X_{m}^{\prime} X_{m}\left(\phi_{m}-\widehat{\boldsymbol{\phi}}_{m}\right)+\left(\mathbf{Z}_{m}-\widehat{\mathbf{Z}}_{m}\right)^{\prime}\left(\mathbf{Z}_{m}-\widehat{\mathbf{Z}}_{m}\right)$. 
Podemos notar, neste caso que a densidade a posteriori conjunta não é uma densidade padrão, portanto só podemos avaliar as densidades a posteriori marginais por meio de métodos de aproximação, como o método de Laplace ou usando métodos de aproximação de Monte Carlo em Cadeia de Markov, como Gibbs Sampling e Metropolis-Hastings. Neste trabalho, optamos pelos métodos de simulação, então encontramos a seguir as densidades condicionais para os algoritmos.

\subsubsection{Densidades a Posteriori Condicionais para $\tau_{m}$ e $\phi_{m}$}

A densidade de $\boldsymbol{\tau}_{m}$, condicionada a $\phi_{m}$ e aos dados, $\mathbf{Z}_{m}$ é dada por,

$$
\Pi_{m}\left(\tau_{m} \mid \phi_{m}, \mathbf{z}_{m}\right) \sim \Gamma\left(\frac{\left(n_{m}-p_{m}+2 \alpha_{m}\right)}{2}, \beta_{m}+\frac{1}{2} B_{m}\right)
$$

Notamos que a distribuição condicional de $\tau_{m}(3.25)$ tem forma conhecida, ou seja, tem distribuição de probabilidade Gama.

A densidade condicional de $\phi_{m}$, condicionada aos dados, $\mathbf{Z}_{m}$ e $\tau_{m}$, é dada por,

$$
\begin{aligned}
\Pi_{m}\left(\phi_{m} \mid \tau_{m}, \mathbf{Z}_{m}\right) \propto & \tau_{m}^{\frac{\lambda_{m}}{2 m_{m}+\alpha_{m}}-1} \exp \left\{-\tau_{m}[\overbrace{m}^{\prime}+\frac{1}{2}\left(\phi_{m}-\widehat{\phi}_{m}\right)^{\prime} X_{m}^{\prime} X_{m}\left(\phi_{m}-\widehat{\phi}_{m}\right)]\right\} \times \\
& \times \Pi_{1}\left(\phi_{m}\right)
\end{aligned}
$$

A distribuição condicional de $\phi_{m}$ (3.26) não tem forma padronizada, ou seja, não tem distribuição de probabilidade conhecida. Quando isso ocorre, utilizamos o algoritmo de Metropolis-Hastings para gerar os parâmetros $\phi_{m}$.

Neste caso, a densidade condicional $\Pi_{m}(t)$ pode ser escrita como $\Pi_{m}(t) \propto$ $h_{m}(t) \Psi_{m}(t)$, onde $h_{m}(t)$ é uma densidade com forma padronizada e $\Psi_{m}(t)$ é uma 
função uniformemente limitada, usamos $h_{m}(t)$ como núcleo para gerar os candidatos do parâmetro de interesse.

Assim, para $\phi_{m}$ pelo algoritmo de Metropolis-Hastings, identificamos o núcleo de transição $h_{m}(t)$ com forma padronizada, o qual é identificado para gerar candidatos para os parâmetros $\phi_{m}$. Neste caso, através da equação (3.26), é identificado um núcleo com a forma da distribuição Gama. Portanto, a densidade condicional pode ser reescrita por,

$$
\left.\Pi_{m}\left(\phi_{m} \mid \tau_{m}, Z_{m}\right) \propto \prod_{\prod_{1}\left(\phi_{m}\right.}^{\operatorname{li}_{m}}\right) \Psi_{m}\left(\phi_{m}\right)
$$

onde $\Psi_{m}\left(\phi_{m}\right) \propto \tau_{m}^{\frac{n_{m}-p_{m}+2 \alpha_{m}}{2}-1} \exp \left\{-\tau_{m}\left[\beta_{m}+\frac{1}{2}\left(\phi_{m}-\widehat{\phi}_{m}\right)^{\prime} X_{m}^{\prime} X_{m}\left(\phi_{m}-\widehat{\phi}_{m}\right)\right]\right\}$. 


\section{CAPÍTULO 4}

\section{Densidade Preditiva}

Em muitas ocasiões, dada a informação amostral, podemos ter interesse em fazer inferência sobre algum valor que ainda não foi observado, isto é, fazer uma previsão para um valor futuro. Na análise Bayesiana, a função densidade de probabilidade para um valor ainda não observado, dada a informação amostral, é conhecido como densidade preditiva para o valor futuro, $W$.

Seja $\mathbf{Z}_{m}=\left(Z_{t(1, m)}, \ldots, Z_{t(n, m)}\right)^{\prime}$ um vetor de valores observados da série. A densidade preditiva para um valor futuro $W$ é dada por,

$$
\begin{aligned}
\Pi_{m}\left(W \mid \mathbf{Z}_{m}\right)= & \int_{\phi_{1}, m} \ldots \int_{\phi_{p_{m}}, m} \int_{\tau_{m}} \Pi_{m}\left(W \mid \phi_{m}, \tau_{m}, \mathbf{Z}_{m}\right) \times \\
& \times \Pi_{m}\left(\phi_{m}, \tau_{m} \mid \mathbf{Z}_{m}\right) d \phi_{1, m} \ldots d \phi_{p_{m}, m} d \tau_{m}
\end{aligned}
$$

Desta forma, $\Pi_{m}\left(W \mid \mathbf{Z}_{m}\right)=E\left(\Pi_{m}\left(W \mid \phi_{m}, \boldsymbol{\tau}_{m}, \mathbf{Z}_{m}\right)\right)$ onde $\Pi_{m}\left(W \mid \phi_{m}, \boldsymbol{\tau}_{m}, \mathbf{Z}_{m}\right)$ é a função densidade de $W$ condicionada no conhecimento dos parâmetros $\phi_{m}, \tau_{m}$ e os dados $\mathbf{Z}_{m}$ e $\Pi_{m}\left(\phi_{m}, \tau_{m} \mid \mathbf{Z}_{m}\right)$ é a densidade a posteriori dos parâmetros.

\subsection{Densidade Preditiva um Passo a Frente}

Como visto acima. a distribuição preditiva Bayesiana é a densidade de valores futuros condicionada as observações passadas. Vamos determinar a densidade pred- 
itiva um passo a frente. $Z_{t(n+1,1)}$, por exemplo, para o mês de janeiro $m=1$ condicionado as observaçoes $\mathbf{Z}_{1}=\left(Z_{t(1,1)}, \ldots, Z_{t(n, 1)}\right)^{\prime}$, portanto,

$$
\begin{aligned}
\Pi\left(Z_{t(n+1,1)} \mid \mathbf{Z}_{1}\right)= & \int_{\phi_{1}, 1} \ldots \int_{\phi_{p_{1}, 1}} \int_{\tau_{1}} \Pi\left(Z_{t(n+1,1)} \mid \phi_{1,1}, \ldots, \phi_{p_{1}, 1}, \tau_{1}, \mathbf{Z}_{1}\right) \times \\
& \times \Pi\left(\phi_{1,1}, \ldots, \phi_{p_{1}, 1}, \tau_{1} \mid Z_{1}\right) d \phi_{1,1} \ldots d \phi_{p_{1}, 1} d \tau_{1}
\end{aligned}
$$

isto é,

$$
\Pi\left(Z_{t(n+1,1)} \mid \mathbf{Z}_{1}\right)=E\left(\Pi\left(Z_{t(n+1,1)} \mid \phi_{1,1}, \ldots, \phi_{p_{1}, 1}, \tau_{1}, \mathbf{Z}_{1}\right)\right)
$$

Podemos notar que as integrais da equação (4.2) são analiticamente difíceis de se resolver e muitas vezes necessitam de integração numérica. Uma alternativa é utilizar o método de integração de Monte Carlo.

A previsão um passo a frente é estimada utilizando-se as amostras geradas pelos algoritmos de Gibbs e Metropolis-Hastings, e é dada por,

$$
\begin{gathered}
E\left(Z_{t(n+1,1)} \mid \mathbf{Z}_{1}\right)=\int_{-\infty}^{+\infty} Z_{t(n+1,1)} \Pi\left(Z_{t(n+1,1)} \mid \mathbf{Z}_{1}\right) d Z_{t(n+1,1)}, \\
E\left(Z_{t(n+1,1)} \mid \mathbf{Z}_{1}\right)=\int_{-\infty}^{+\infty} Z_{t(n+1,1)}\left[\int_{\phi_{1}, 1} \ldots \int_{\phi_{p_{1}}, 1} \int_{\tau_{1}} \Pi\left(Z_{t(n+1,1)} \mid \phi_{1,1}, \ldots, \phi_{p_{1}, 1}, \tau_{1}, \mathbf{Z}_{1}\right) \times\right. \\
\left.\times \Pi\left(\phi_{1,1}, \ldots, \phi_{p_{1}, 1}, \tau_{1} \mid \mathbf{Z}_{1}\right) d \phi_{1,1} \ldots d \phi_{p_{1}, 1} d \tau_{1}\right] d Z_{t(n+1,1)}
\end{gathered}
$$

alterando a ordem das integrações temos,

$$
\begin{aligned}
E\left(Z_{t(n+1,1)} \mid \mathbf{Z}_{1}\right)= & \int_{\phi_{1}, 1} \ldots \int_{\phi_{p_{1}, 1}} \int_{\tau_{1}} E\left(Z_{t(n+1,1)} \mid \phi_{1,1}, \ldots, \phi_{p_{1}, 1}, \tau_{1}, \mathbf{Z}_{1}\right) \times \\
& \times \Pi\left(\phi_{1,1}, \ldots, \phi_{p_{1}, 1}, \tau_{1} \mid \mathbf{Z}_{1}\right) d \phi_{1,1} \ldots d \phi_{p_{1}, 1} d \tau_{1}
\end{aligned}
$$


Um estimador de Monte Carlo para (4.4), considerando-se as amostras geradas pelo algoritmo de Gibbs com Metropolis, é dado por,

$$
\widehat{E}\left(Z_{t(n+1,1)} \mid \mathbf{Z}_{1}\right)=\frac{1}{M} \sum_{j=1}^{M} E\left(Z_{t(n+1,1)} \mid \phi_{1,1}^{(j)}, \ldots, \phi_{p_{1}, 1}^{(j)}, \tau_{1}^{(j)}, \mathbf{Z}_{1}\right)
$$

Podemos verificar de (4.5) a simplicidade no cálculo da previsão devido ao uso dos algoritmos computacionais de MCMC.

Usando o modelo $\operatorname{PAR}\left(p_{1}\right)$, temos que

$$
Z_{t(n+1,1)}=\sum_{i=1}^{p_{1}} \phi_{i, 1} Z_{t(n+1,1)-i}+a_{t(n-1,1)}, \quad \quad a_{t(n-1,1)} \sim N\left(0, \tau_{1}^{-1}\right) .
$$

A função densidade de probabilidade de $Z_{t(n+1,1)}$, condicionada a todos os parâmetros do modelo e observações passadas é dada por

$$
\begin{aligned}
& \Pi_{1}\left(Z_{t(n+1,1)} \mid \phi_{1,1}, \ldots \phi_{p_{1}, 1} \tau_{1}, \mathbf{Z}_{1}\right) \propto \tau_{1}^{1 / 2} \exp \left\{-\frac{\tau_{1}}{2}\left(Z_{t(n+1,1)}-\phi_{1,1} Z_{t(n+1,1)-1}-\right.\right. \\
& \left.\left.-\ldots-\phi_{p_{1}, 1} Z_{t(n+1,1)-p_{1}}\right)^{2}\right\}
\end{aligned}
$$

A densidade preditiva Bayesiana de $Z_{t(n+1,1)}$, dados $\phi_{1,1}, \ldots \phi_{p_{1}, 1}, \tau_{1}$ e $\mathbf{Z}_{1}$, tem valor esperado dado por

$$
E\left(Z_{t(n+1,1)} \mid \phi_{1,1}, \ldots \phi_{p_{1,1},} \tau_{1}, Z_{1}\right)=\phi_{1,1} Z_{t(n+1,1)-1}^{\prime}+\ldots+\phi_{p_{1}, 1} Z_{t(n+1,1)-p_{1}}
$$

sendo $t=t(r, m)=(r-1) s+m$, notamos que $Z_{t(n+1,1) .}=Z_{n s+1}$ e os valores passados são $Z_{t(n+1,1)-1}=Z_{n s}, Z_{t(n+1,1)-2}=Z_{n s-1}, \ldots, Z_{t(n+1,1)-p_{1}}=Z_{n s-p_{1+1}}$

Desta forma. temos

$$
59 \times 12
$$

$$
E\left(Z_{t(n+1,1)} \mid \phi_{1,1}^{(j)}, \ldots \phi_{p_{1,1},}^{(j)} \tau_{1}^{(j)}, Z_{1}\right)=\phi_{1,1}^{(j)} Z_{n s}+\ldots+\phi_{p_{1}, 1} Z_{n s-p_{1}}
$$


Substituindo (4.6) na equação (4.5) mostramos a previsão do valor $Z_{t(n+1,1)}$ pode ser facilmente calculado usando-se o método de simulação de Monte Carlo.

\subsection{Seleção de Modelos}

Sob o enfoque Bayesiano, a seleção de modelos pode ser conduzida através de muitas estratégias propostas na literatura (ver por exemplo, Jeffreys, 1939; Geisser e Edy, 1979). Destacamos neste trabalho, uma técnica que utiliza densidades preditivas ordenadas.

Podemos plotar $c_{t(r, m)}=\widehat{\Pi}\left(Z_{t(r, m)} \mid \mathbf{Z}_{1}\right)$ versus $t(r, m), r=1, \ldots, n$ para cada modelo proposto para cada mês $m$. O modelo que apresentar, os maiores valores de $c_{t(r, m)}$ é o melhor. O critério da preditiva Bayesiana, seleciona o modelo que maximiza o produto $c(l)=\prod_{r=1}^{n} c_{t(r, m)}(l)$, onde $l$ indexa o $l$-ésimo modelo autoregressivo ( por exemplo, $l=1$ significa que podemos estar calculando $c_{t(r, m)}$ para o modelo PAR(1) para um certo mês $m$ ). No capítulo de resultados esse critério é empregado para escolha da ordem do modelo para os meses $m=1, \ldots, 12$. 


\section{CAPÍTULO 5}

\section{Aplicações}

Após apresentarmos nos capítulos anteriores as abordagens Clássica e Bayesiana para modelos $\operatorname{PAR}\left(p_{m}\right)$, vamos apresentar neste capítulo a título de ilustração, alguns exemplos de aplicações. Apresentamos primeiramente uma aplicação do teste estatístico para verificar a existência de periodicidade na função de autocorrelação de séries temporais. O método proposto é ilustrado para duas séries estacionárias geradas, para duas séries sazonais geradas e para duas séries com dados de vazões.

\subsection{Aplicação do Teste de Sazonalidade}

Exemplo 1. Série gerada por um modelo AR(1) estacionário, dado por:

$$
Z_{t}=0.8 Z_{t-1}+a_{t}, \quad a_{t} \sim N(0,4) .
$$

Exemplo 2. Série gerada por um modelo AR(2) estacionário, dado por:

$$
Z_{t}=-0.5 Z_{t-1}+0.3 Z_{t-2}+a_{t}, \quad a_{t} \sim N(0,4) .
$$

Para estes dois exemplos acima temos séries estacionárias, portanto espera-se que a hipótese $H_{0}: \rho_{m}(j)$ é constante em $m$, não seja rejeitada. 
Exemplo 3. Série gerada por um modelo PAR(1), dado por:

$$
Z_{t(r, m)}=\phi_{1, m} Z_{t(r, m)-1}+a_{t(r, m)}, \quad \quad a_{t(r, m)} \sim N\left(0, \sigma_{t(r, m)}^{2}\right)
$$

cujos valores dos parâmetros são dados na Tabela 1.

Tabela 1. Parâmetros do Exemplo 3

\begin{tabular}{|ll|lc|}
\hline \multicolumn{2}{|c|}{$\sigma_{\mathrm{t}(\mathrm{r}, \mathrm{m})}$} & \multicolumn{2}{c|}{$\phi_{1, \mathrm{~m}}$} \\
\hline 0.8453 & 1.0929 & 0.5776 & 0.1378 \\
0.7250 & 0.9463 & 0.6860 & 0.0621 \\
0.7405 & 0.9422 & 0.6927 & -0.1275 \\
0.9660 & 0.9686 & 0.4582 & 0.2498 \\
0.8351 & 0.8894 & 0.3868 & 0.2314 \\
0.9160 & 0.9951 & 0.2998 & 0.0977 \\
\hline
\end{tabular}

Exemplo 4. Série gerada por um modelo PAR(2), dado por:

$Z_{t(r, m)}=\phi_{1, m} Z_{t(r, m)-1}+\phi_{2, m} Z_{t(r, m)-2}+a_{t(r, m)}, \quad \quad a_{t(r, m)} \sim N\left(0, \sigma_{t(r, m)}^{2}\right)$

onde os parâmetros são dados na Tabela 2.

Tabela 2. Parâmetros do Exemplo 4

\begin{tabular}{|cc|cccc|}
\hline \multicolumn{2}{|c|}{$\sigma^{2}{ }_{\mathrm{t}(\mathrm{r}, \mathrm{m})}$} & \multicolumn{2}{c}{$\phi_{1, \mathrm{~m}}$} & & \multicolumn{2}{c|}{$\phi_{2, \mathrm{~m}}$} \\
\hline 0.196 & 0.296 & 1.132 & 0.900 & -0.638 & -0.1665 \\
0.212 & 0.227 & 0.889 & 0.217 & -0.057 & -0.0214 \\
0.200 & 0.150 & 0.722 & 0.331 & 0.0231 & -0.3773 \\
0.174 & 0.161 & 0.593 & 0.829 & 0.0735 & -0.3921 \\
0.164 & 0.124 & 0.798 & 0.733 & -0.0343 & 0.0952 \\
0.155 & 0.153 & 0.694 & 0.966 & 0.1443 & -0.3854 \\
\hline
\end{tabular}


Exemplo 5. Série com dados reais de vazões médias mensais em $\left(\mathrm{m}^{3} / \mathrm{s}\right)$ que chegam ao reservatório da hidroelétrica de Furnas no sudeste do Brasil. O modelo ajustado para esta série foi um $\operatorname{PAR}(2)$, dado por:

$$
Z_{t(r, m)}=\phi_{1, m} Z_{t(r, m)-1}+\phi_{2, m} Z_{t(r, m)-2}+a_{t(r, m)}, \quad \quad a_{t(r, m)} \sim N\left(0, \sigma_{t(r, m)}^{2}\right)
$$

onde os valores estimados dos parâmetros são dados na Tabela 3.

Tabela 3. Parâmetros do Exemplo 5

\begin{tabular}{|cc|cccc|}
\hline \multicolumn{2}{|c|}{$\sigma^{2}{ }_{\mathrm{t}(\mathrm{r}, \mathrm{m})}$} & \multicolumn{2}{c|}{$\phi_{\mathrm{l}, \mathrm{m}}$} & \multicolumn{2}{c|}{$\phi_{2, \mathrm{~m}}$} \\
\hline 562.1258 & 60.8756 & 0.6363 & 0.6181 & -0.0891 & 0.3502 \\
562.6235 & 51.2301 & 0.6343 & 0.8607 & -0.1755 & 0.0732 \\
513.8956 & 139.9743 & 0.4125 & 0.5061 & 0.2729 & 0.3435 \\
233.1583 & 149.8997 & 0.6178 & 0.4401 & 0.2195 & 0.3721 \\
121.6653 & 200.8616 & 0.6738 & 0.7912 & 0.2539 & -0.0277 \\
142.5464 & 360.5217 & 0.6869 & 0.3427 & 0.2092 & 0.3916 \\
\hline
\end{tabular}

Exemplo 6. Série com dados reais de vazões médias mensais da barragem de Emborcação. O modelo ajustado para esta série foi um PAR(1), dado por:

$$
Z_{t(r, m)}=\phi_{1, m} Z_{t(r, m)-1}+a_{\ell(r, m),} \quad a_{t(r, m)} \sim N\left(0, \sigma_{t(r, m)}^{2}\right)
$$

onde os valores estimados dos parâmetros são dados na Tabela 4. 
Tabela 4. Parâmetros do Exemplo 6

\begin{tabular}{|cc|cc|}
\hline \multicolumn{2}{|c|}{$\sigma_{\mathrm{t}(\mathrm{r}, \mathrm{m})}^{2}$} & \multicolumn{2}{|c|}{$\phi_{1, \mathrm{~m}}$} \\
\hline 320.0884 & 30.4095 & 0.3976 & 0.9033 \\
315.5035 & 28.7939 & 0.6455 & 0.8642 \\
321.5991 & 33.8129 & 0.4660 & 0.7358 \\
185.0837 & 62.2437 & 0.6234 & 0.4005 \\
72.4676 & 148.4024 & 0.8159 & 0.4471 \\
52.2730 & 267.3461 & 0.8254 & 0.5095 \\
\hline
\end{tabular}

Para as séries $3,4,5$ e 6 respectivamente temos séries periódicas, com $r=60$ anos, $m=1, \ldots, s$ e $s=12$, podemos constatar que as funções de autocorrelações $\rho_{m}(j)$ são periódicas, ou seja, rejeitamos $H_{0}$ nestes exemplos para todos os 12 meses, como mostra a Tabela 5.

As Figuras 2 e 3 apresentam o comportamento das funções de autocorrelações para os passos $j=1,2,3$, quando utlizamos os dados gerados pelo modelo $\operatorname{AR}(2)$ do exemplo 2 e os dados reais do exemplo 5 de Furnas respectivamente. Podemos observar que para a série estacionária as funções de autocorrelações apresentam um comportamento constante para todos os passos, ao contrário do que observamos para a série sazonal de Furnas. 


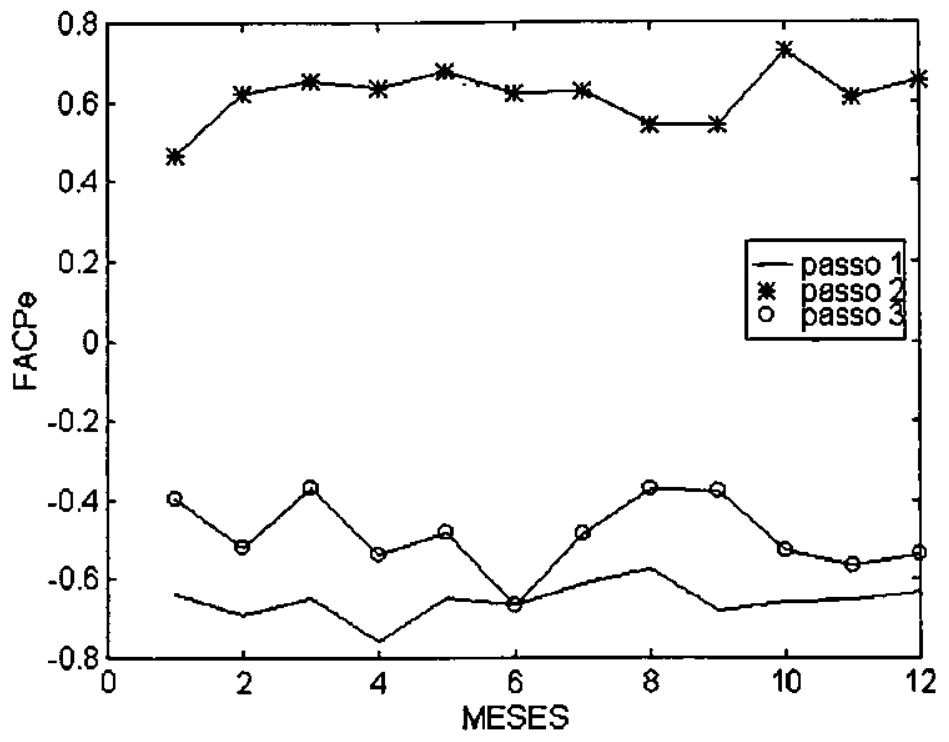

Figura 2. Funções de Autocorrelações para a série gerada AR(2).

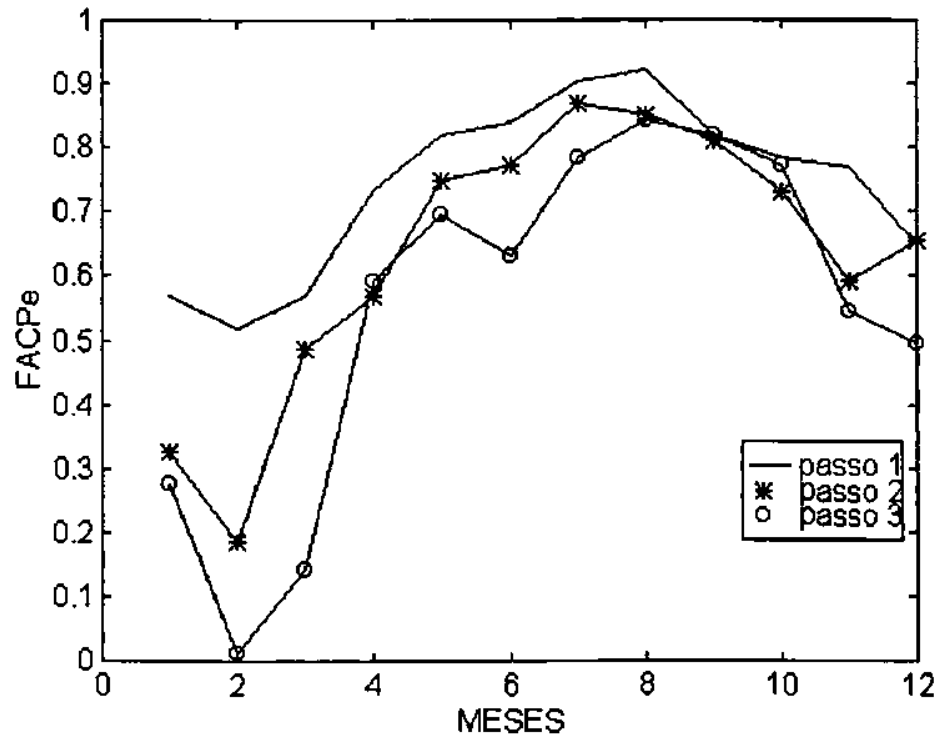

Figura 3. Funções de Autocorrelações para a série de Furnas. 
A Tabela 5 apresenta os valores da estatística teste $\chi_{s-1}^{2}$, para os passos $j=1,2, \ldots, 6$, comparamos estes valores com os valores críticos para os níveis de significância $5 \%$ e $10 \%$ respectivamente.

Tabela 5. Resultados da estatística teste $\chi_{11}^{2}$ para as séries dos exemplos

\begin{tabular}{|c|c|c|c|c|c|c|}
\hline & \multicolumn{7}{|c|}{ Passos } \\
\hline Séries & $\mathbf{1}$ & $\mathbf{2}$ & $\mathbf{3}$ & $\mathbf{4}$ & $\mathbf{5}$ & $\mathbf{6}$ \\
\hline $\mathrm{AR}(1)$ & 1.88 & 3.11 & $\mathbf{8 . 5 0}$ & 9.83 & $\mathbf{8 . 5 1}$ & 3.78 \\
\hline $\mathrm{AR}(2)$ & 4.09 & 7.71 & 12.45 & 7.52 & 16.65 & 15.4 \\
\hline PAR(1) & 211.6759 & 140.6980 & 58.1761 & 56.6471 & 22.5462 & 49.9359 \\
\hline PAR(2) & 122.19 & 317.77 & 239.76 & 163.32 & 64.99 & 26.36 \\
\hline Furnas & 40.82 & 82.63 & 122.88 & 113.16 & 93.70 & 72.36 \\
\hline Emborcação & 86.9741 & 172.1337 & 215.9244 & 150.5312 & 106.2123 & 81.7433 \\
\hline
\end{tabular}

Considerando os valores críticos $\chi_{11}^{2}(0.05)=19,6751$ e $\chi_{11}^{2}(0.1)=24,7250$ podemos observar que o teste confirma a estacionariedade das séries geradas pelos modelos $A R(1)$ e $A R(2)$, ou seja, não se rejeita a hipótese $H_{0}$ de que as funções de autocorrelações $\rho_{m}(j)$ são constantes em $m$. Para as demais séries comprovamos a periodicidade, ou seja, as funções de autocorrelações $\rho_{m}(j)$ variam de forma periódica com período $s$. 


\subsection{Aplicações para o Exemplo 5}

Série com dados reais de vazões médias mensais em $\left(\mathrm{m}^{3} / \mathrm{s}\right)$ que chegam ao reservatório da hidroelétrica de Furnas no sudeste do Brasil. Esta é uma série mensal com dados coletados de janeiro de 1931 a dezembro de 1990, tendo portanto 60 anos de dados mensais, ou seja, uma série sazonal com 720 valores observados, $m=1, \ldots, 12, s=12$ e $r=60$. O modelo ajustado para esta série foi um $\operatorname{PAR}(2)$, dado por

$$
Z_{t(r, m)}=\frac{\tilde{Z}_{t(r, m)}-\widehat{\mu}_{m}}{\widehat{\sigma}_{m}}
$$

onde

$$
Z_{t(r, m)}=\phi_{1, m} Z_{t(r, m)-1}+\phi_{2, m} Z_{t(r, m)-2}+a_{t(r, m)}
$$

Nesta seção, aplicamos aos dados da série, a metodologia da análise clássica baseada nas Funções Periódicas proposta no capítulo 2.

A Figura 4 a seguir mostra os gráficos das funções de autocorrelação periódica (PeFAC) e autocorrelação parcial periódica (PeFACP) para os meses de janeiro a dezembro. 

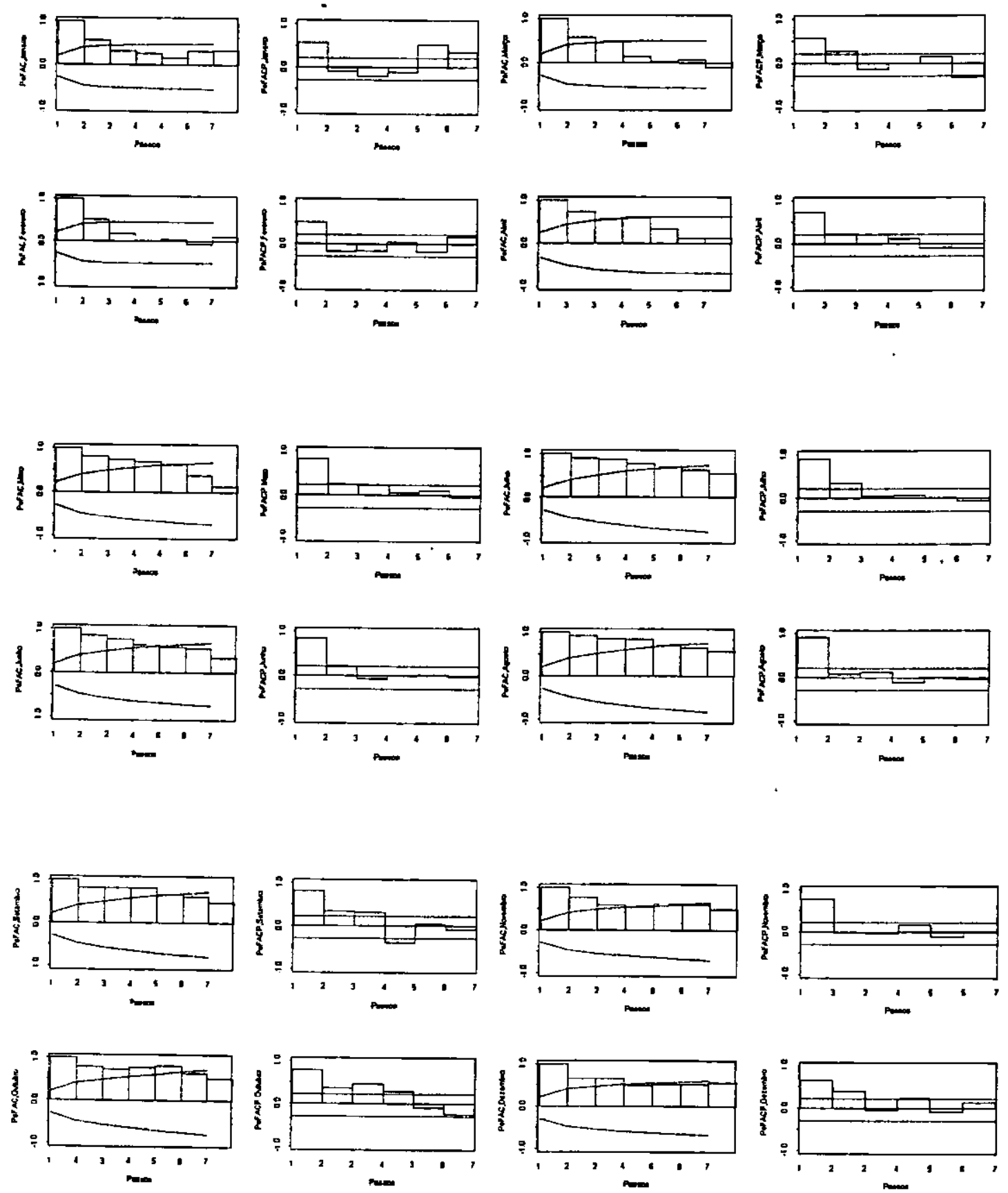

Figura 4. PeFAC e PeFACP para os meses de Janeiro a Dezembro. 
Notamos pelos gráficos das funções PeFAC e PePACF que podemos escolher um modelo $\operatorname{PAR}\left(\mathrm{p}_{m}\right)$, com $p_{m}=1,2$ ou 3 para todos os meses.

Usando o Critério de Informação Bayesiano, BIC os modelos mais parcimoniosos para os meses de Janeiro a Dezembro são apresentados na Tabela 6.

Tabela 6. Valor do BIC e $\mathrm{BIC}_{\mathrm{m}}$ para os meses de Janeiro a Dezembro

\begin{tabular}{l|l|l|l}
\hline & \multicolumn{3}{|c}{ BIC $_{m}$} \\
\hline Meses & $p_{m}=1$ & $p_{m}=2$ & $p_{m}=3$ \\
\hline Janeiro $(m=1)$ & $-0,3845$ & $-0,3163$ & $-0,2480$ \\
Fevereiro $(m=2)$ & $-0,8591$ & $-0,7909$ & $-0,7226$ \\
Março $(m=3)$ & $-1,3427$ & $-1,2745$ & $-1,2063$ \\
Abril $(m=4)$ & $-1,2869$ & $-1,2187$ & $-1,1504$ \\
Maio $(m=5)$ & $-2,1040$ & $-2,0358$ & $-1,9675$ \\
Junho $(m=6)$ & $-2,0510$ & $-1,9828$ & $-1,9145$ \\
Julho $(m=7)$ & $-1,0921$ & $-1,0239$ & $-0,9556$ \\
Agosto $(m=8)$ & $-0,9306$ & $-0,8624$ & $-0,7941$ \\
Setembro $(m=9)$ & $-0,7880$ & $-0,7199$ & $-0,6516$ \\
Outubro $(m=10)$ & $-0,5261$ & $-0,4580$ & $-0,400$ \\
Novembro $(m=11)$ & $-0,2771$ & $-0,2081$ & $-0,1390$ \\
Dezembro $(m=12)$ & $-0,3189$ & $-0,2498$ & $-0,1807$ \\
\hline \multicolumn{1}{c|}{12} & $-11,1171$ & $-10,3303$ \\
B1C= $\sum_{m=1} B I C_{m}$ & $-11,6839$ & & \\
\hline
\end{tabular}


O procedimento Bayesiano usado na identificação do modelo adequado através da densidade preditiva, o valor do produto produto $c(l)$ para os 12 meses indicam a escolha dos melhores modelos. Os resultados obtidos pelo critério de adequabilidade do modelo baseado na preditiva ordenada são apresentados na Tabela 7.

Tabela 7. Seleção de Modelos para a série de Furnas

\begin{tabular}{l|l|l|l}
\hline \multirow{2}{*}{ meses } & $p_{m}=1$ & $p_{m}=2$ & $p_{m}=3$ \\
\cline { 2 - 4 } & $\mathrm{c}(\mathrm{l})$ & $\mathrm{c}(\mathrm{l})$ & $\mathrm{c}(\mathrm{l})$ \\
\hline Janeiro $(\mathrm{m}=1)$ & 0,0011 & 0,0001 & 0,0001 \\
Fevereiro $(\mathrm{m}=2)$ & 0,0037 & 0,0036 & 0,0004 \\
Março $(\mathrm{m}=3)$ & 0,0079 & 0,0150 & 0,0134 \\
Abril $(\mathrm{m}=4)$ & 0,0019 & 0,0057 & 0,0457 \\
Maio $(\mathrm{m}=5)$ & 0,7899 & 1,9978 & 2,6106 \\
Junho $(\mathrm{m}=6)$ & 0,0492 & 0,0727 & 0,0814 \\
Julho $(\mathrm{m}=7)$ & 4,5596 & 23,5917 & 24,1251 \\
Agosto $(\mathrm{m}=8)$ & 5,8455 & 5,5156 & 6,1313 \\
Setembro $(\mathrm{m}=9)$ & 0,0471 & 0,0766 & 0,1715 \\
Outubro $(\mathrm{m}=10)$ & 0,0230 & 0,0305 & 0,1717 \\
Novembro $(\mathrm{m}=11)$ & 0,0665 & 0,0619 & 0,0655 \\
Dezembro $(\mathrm{m}=12)$ & 0,0020 & 0,0050 & 0,0042 \\
\hline
\end{tabular}

Analisando as Tabelas 6 e 7 respectivamente, podemos notar os resultados não coincidem para todos os meses, o que é comum uma vez que o procedimento bayesiano não é parcimonioso, desta maneira é necessário avaliar as funções de autocorrelações periódicas parciais juntamente com os intervalos de credibilidade.

A Figura 5 mostram os gráficos de $c_{t(r, m)}$ versus $t(r, m), r=1, \ldots, n$ para diferentes modelos da série de Furnas para os meses de Janeiro a Dezembro. 


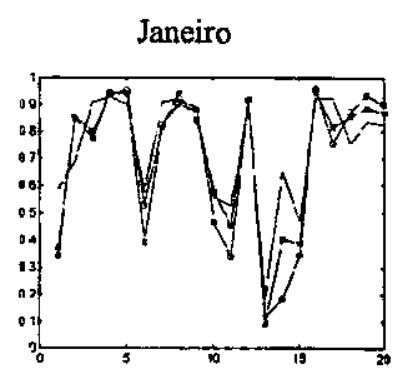

Fevereiro

Março
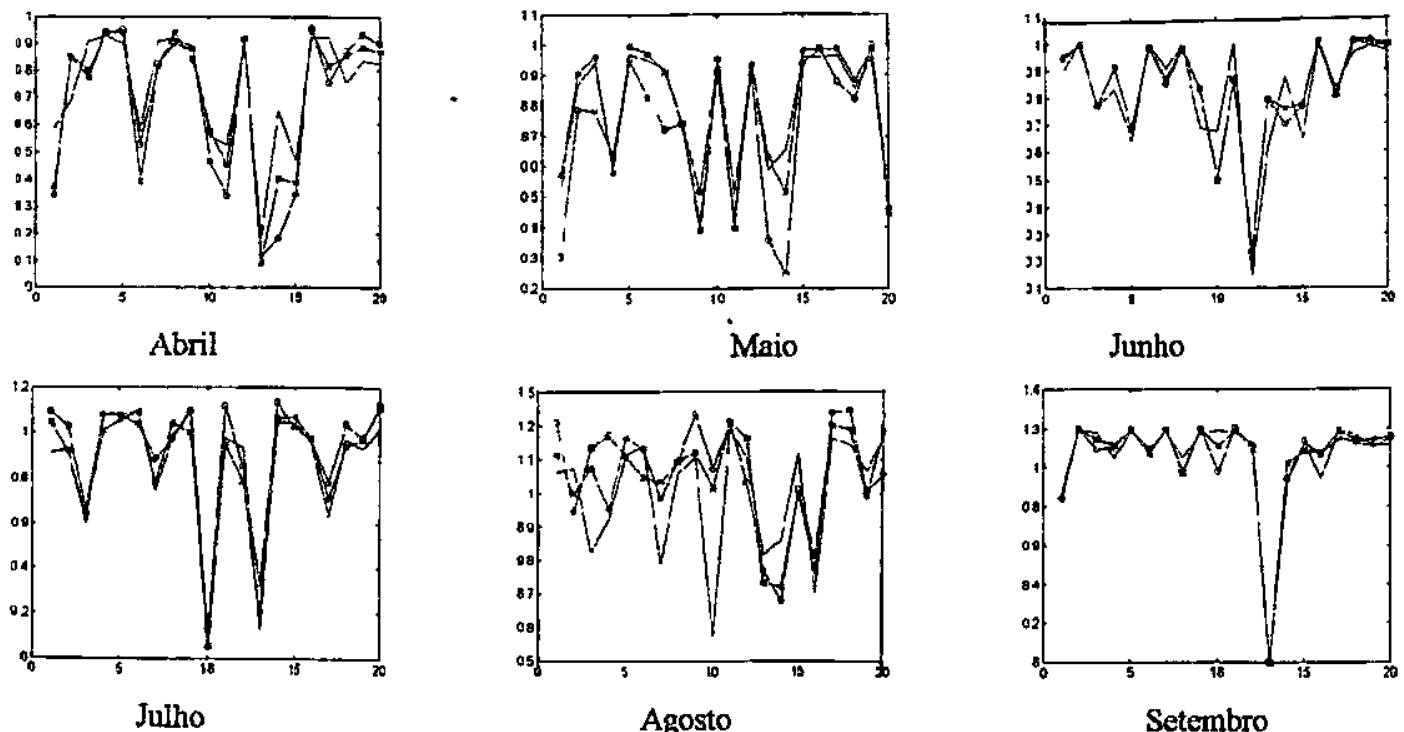

Junho
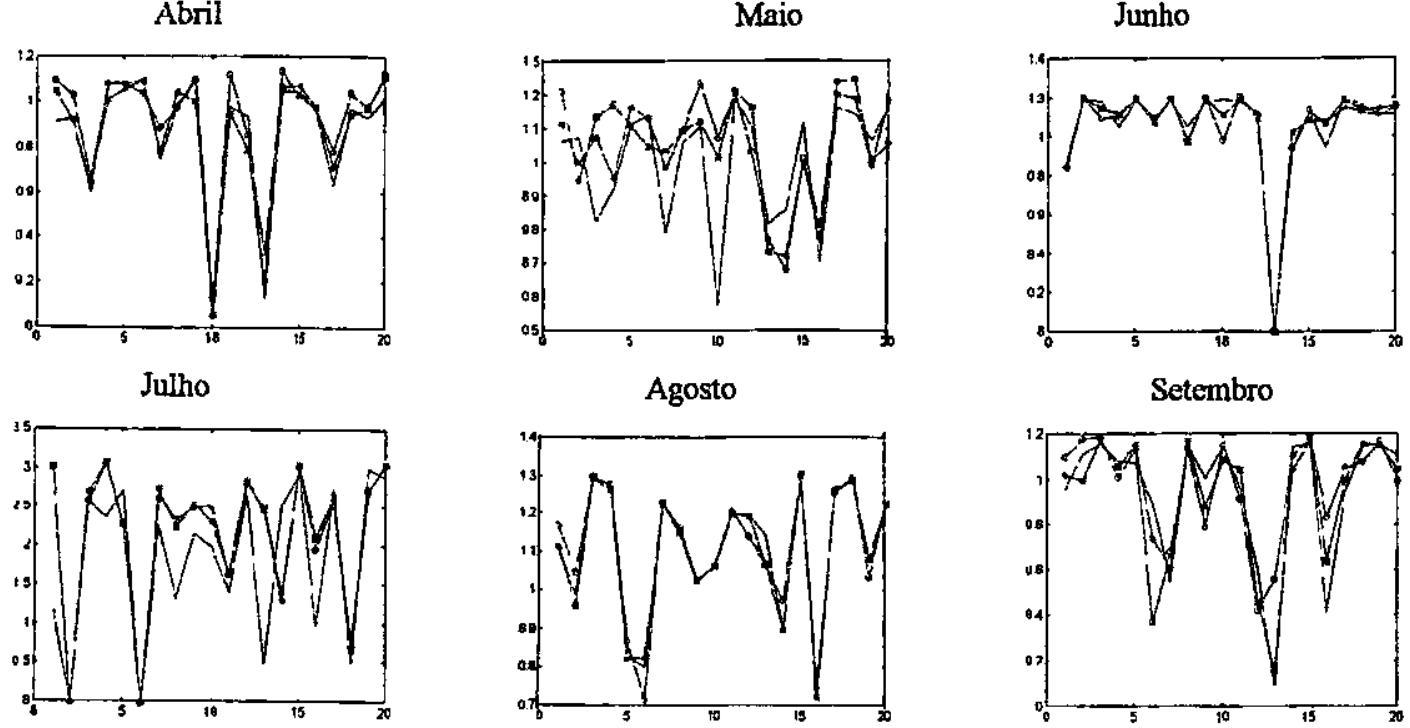

Agosto

Setembro
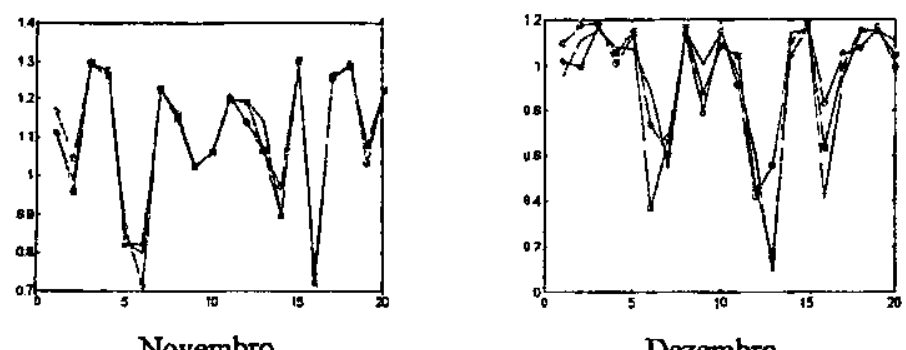

Outubro
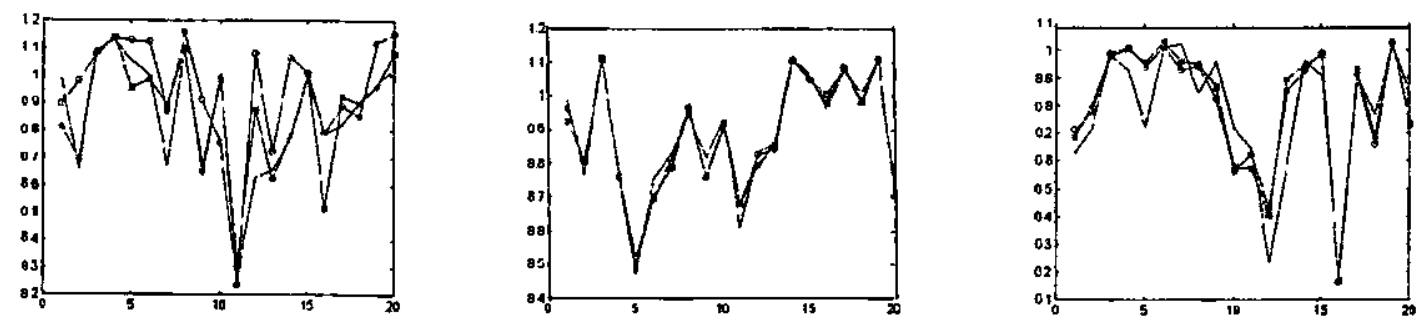

Figura 5. $c_{t(r, m)} \times t(r, m), r=1, \ldots, n$ para diferentes modelos para os meses de Janeiro a Dezembro. 
Como visto, no capítulo 3, em inferência Bayesiana. as inferências são baseadas nas suas densidades a posteriori marginais.

Para inferir dos parâmetros $\phi_{m}$ e $\tau_{m}$, considerando os dados e as densidades a priori não informativa e informativas respectivamente, aproximamos as densidades a posteriori marginais de $\phi_{m}$ e $\tau_{m}$, utilizando o Método de Simulação em Cadeia de Markov.

Utilizando as amostras geradas das densidades condicionais de $\phi_{m}$ e $\tau_{m}$, pelos algoritmos Amostrador de Gibbs e Metroplis-Hastings, construímos os gráficos das densidades a posteriori marginais para os meses de Janeiro a Dezembro, ver figuras 5 a 10 respectivamente.

Nas Figuras 6 e 7 respectivamente, os histogramas mostram as densidades a posteriori marginais para os parâmetros $\phi_{1, m}, \phi_{2, m}$ e $\tau_{m}$, quando consideramos uma priori Não-Informativa de Jeffreys para a série de Furnas.

Nas Figuras 8 e 9, os histogramas mostram respectivamente as densidades a posteriori marginais para os parâmetros $\phi_{1, m}, \phi_{2, m}$ e $\tau_{m}$, quando consideramos uma priori Normal-Gama para a série de Furnas.

Nas Figuras 10 e 11, os histogramas mostram respectivamenteas as densidades a posteriori marginais para os parâmetros $\phi_{1, m}, \phi_{2, m}$ e $\tau_{m}$, quando consideramos uma priori t-student para a série de Furnas. 
Janeiro
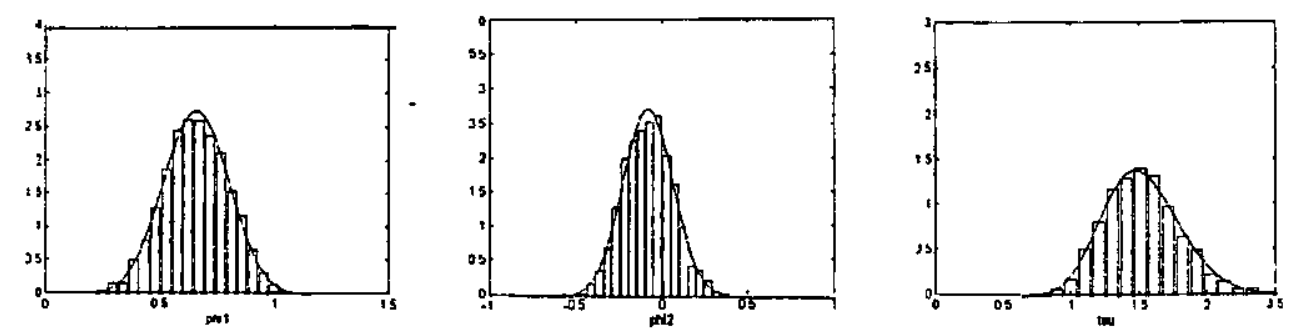

Fevereiro
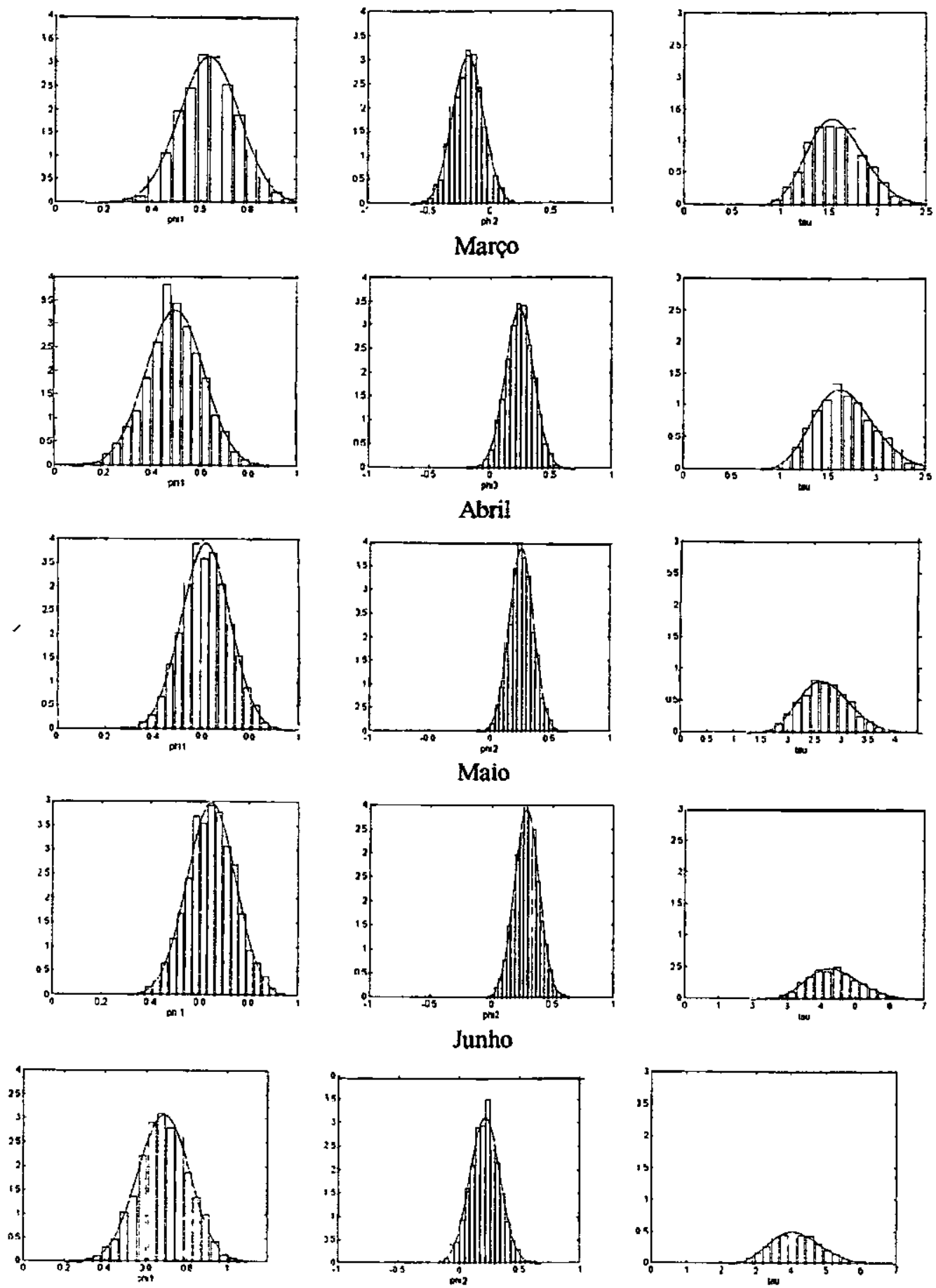

Figura 6. Densidades a Posteriori Marginais para a série de Furnas considerando a priori NãoInformativa para os meses de Janeiro a Junho respectivamente. 
Julho
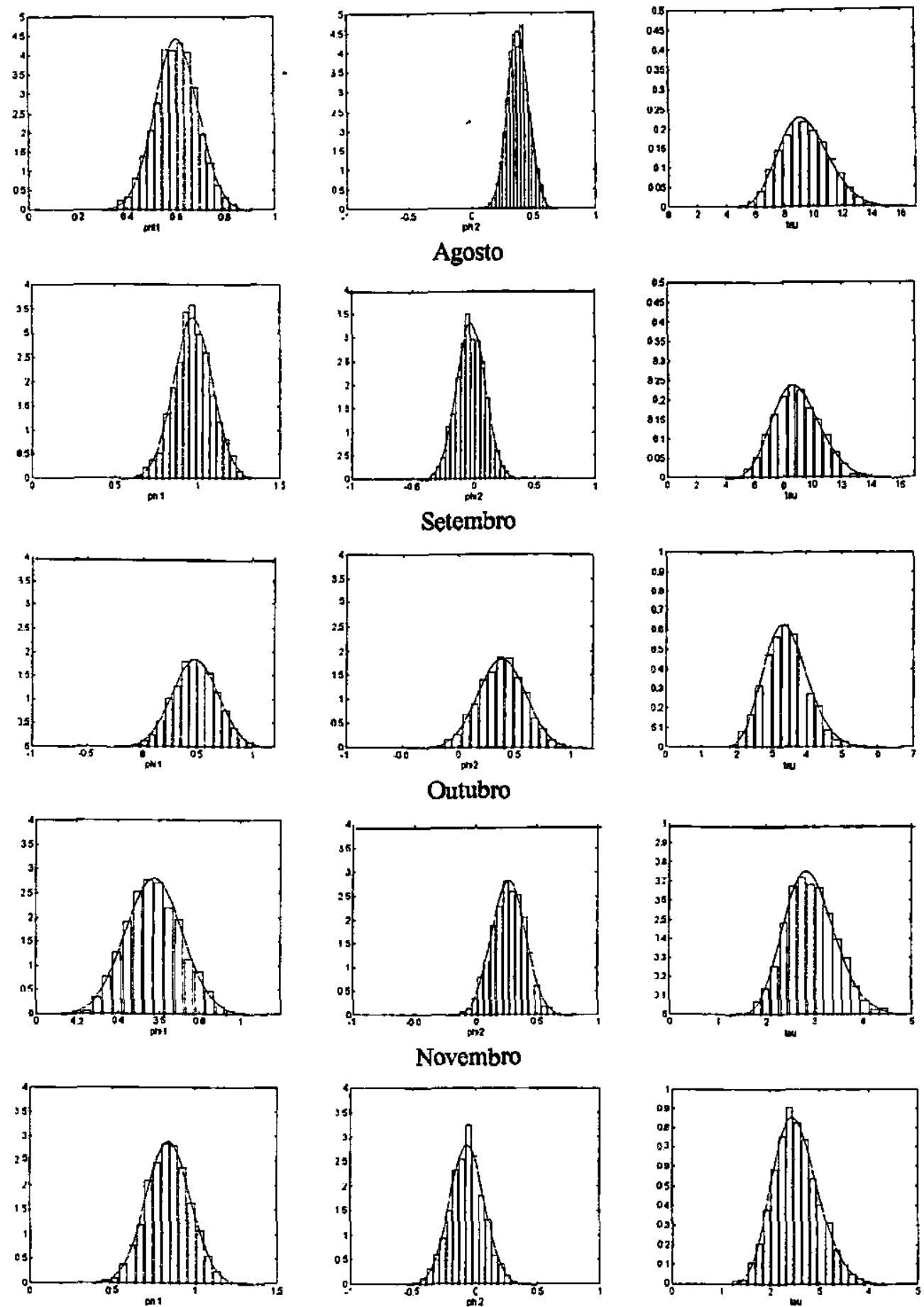

Dezembro
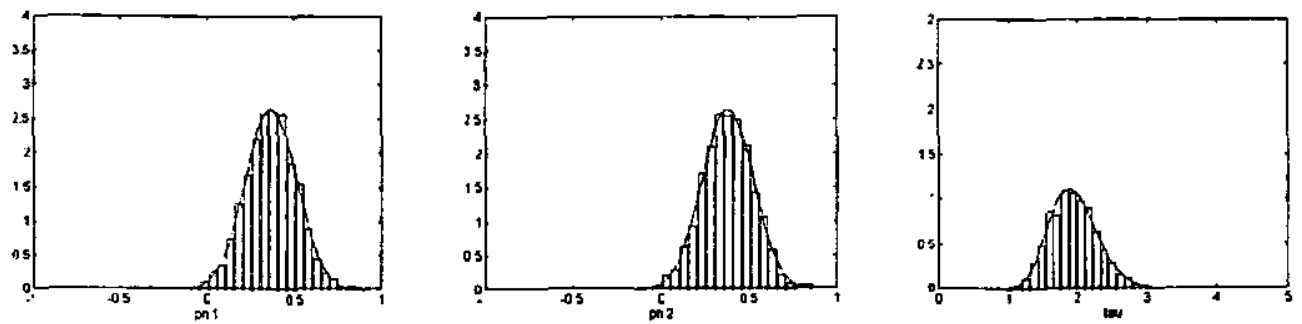

Figura 7. Densidades a Posteriori Marginais para a série de Furnas considerando a priori NãoInformativa para os meses de Julho a Dezembro respectivamente. 
Janeiro
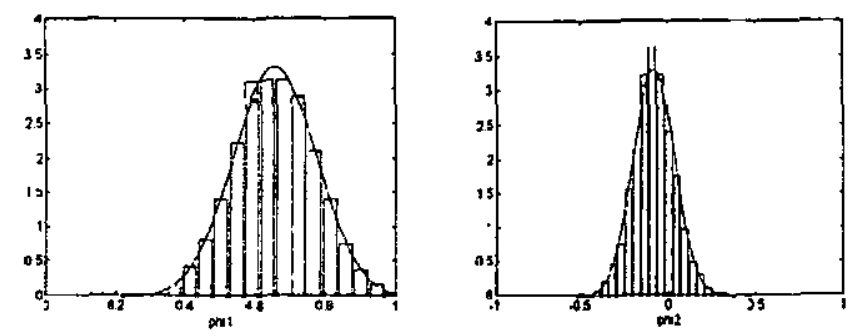

Fevereiro
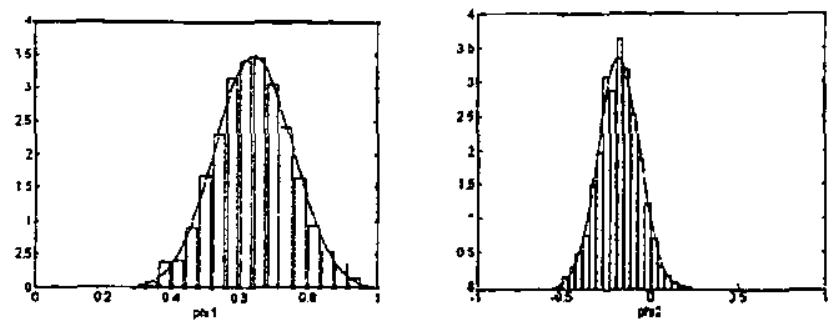

Março
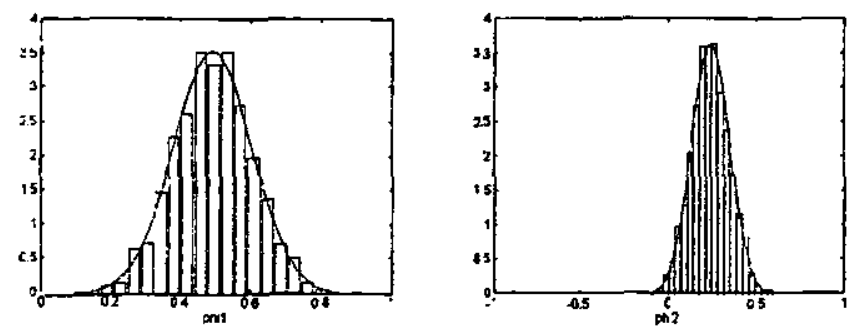

Abril
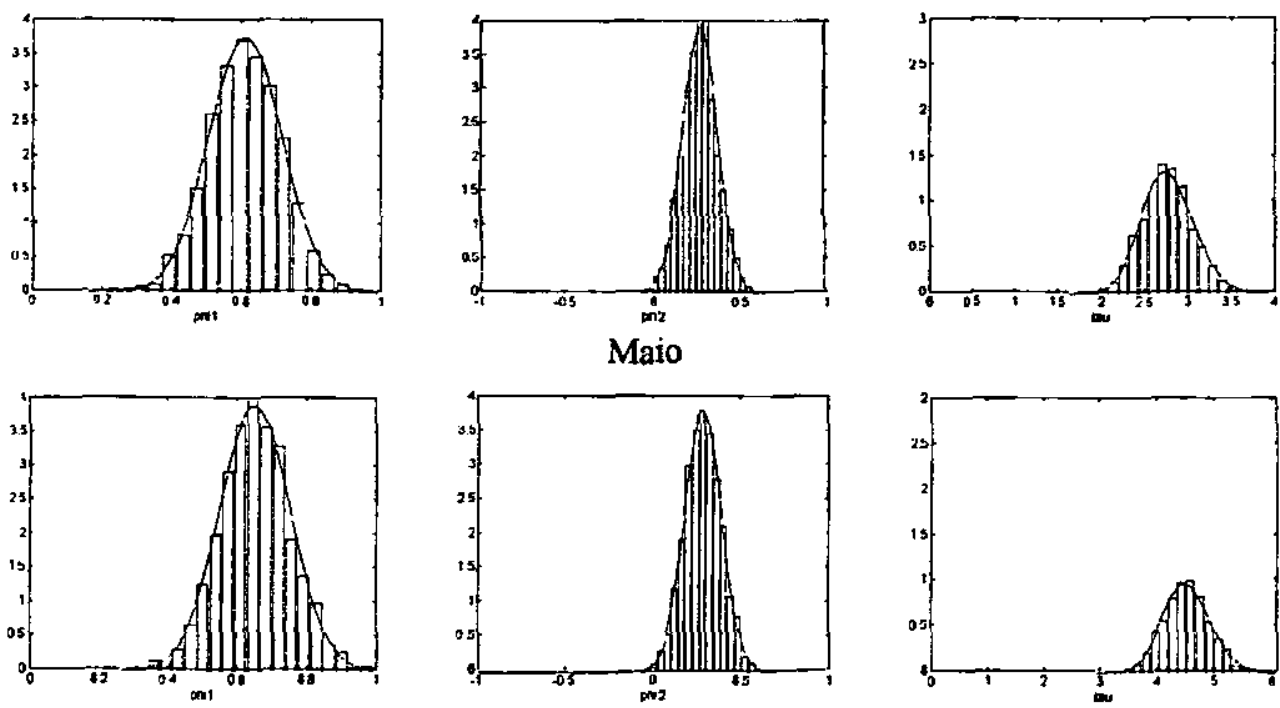

Junho
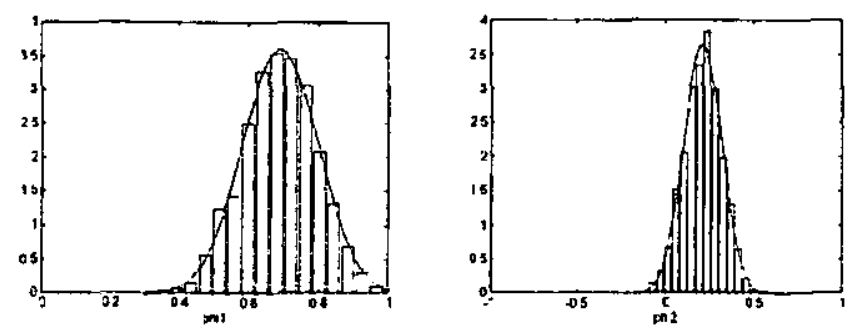

Figura 8. Densidades a Posteriori Marginais para a série de Furnas considerando a priori Normal- Gama para os meses de Janeiro a Junho respectivamente. 
Julho
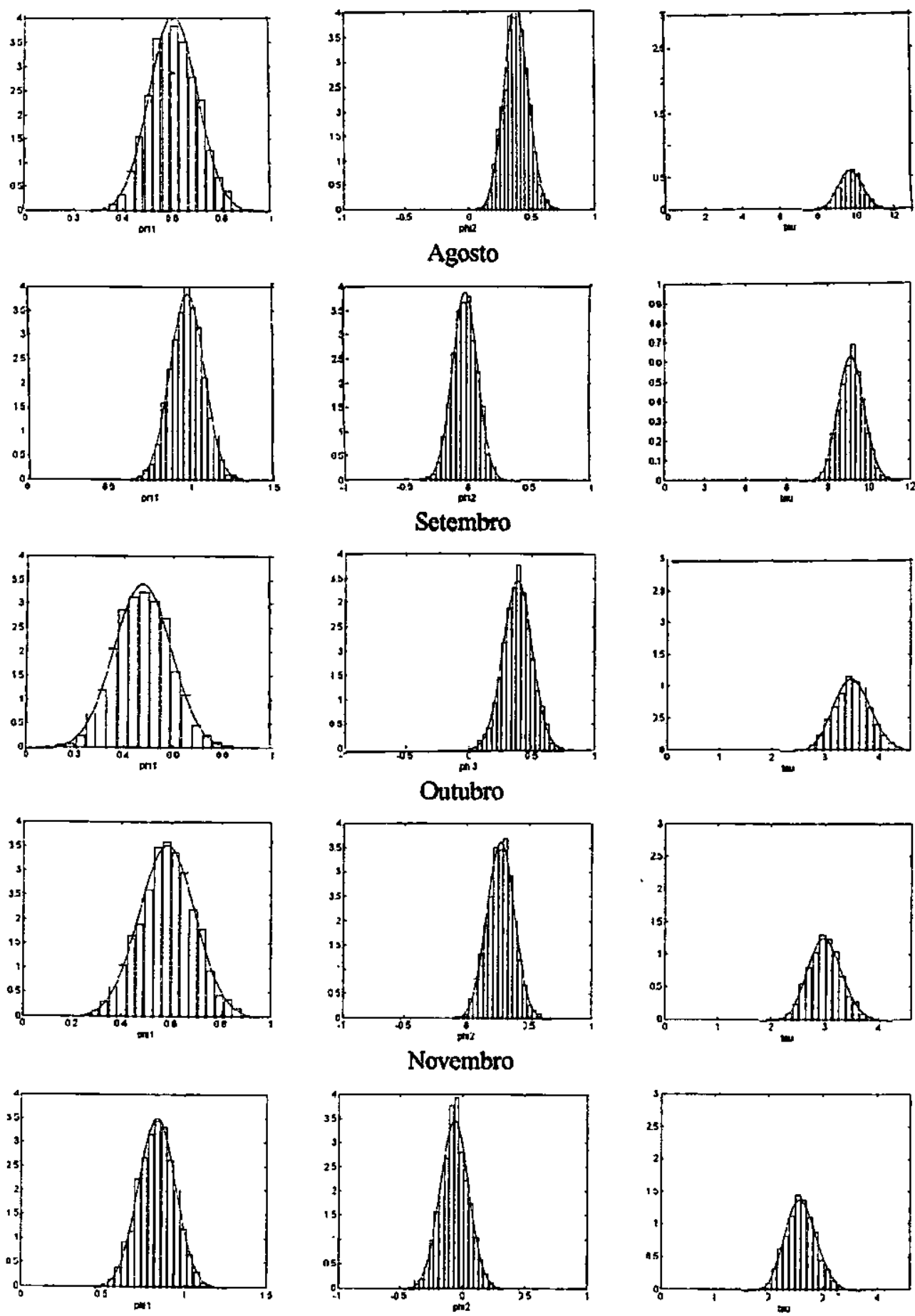

Dezembro
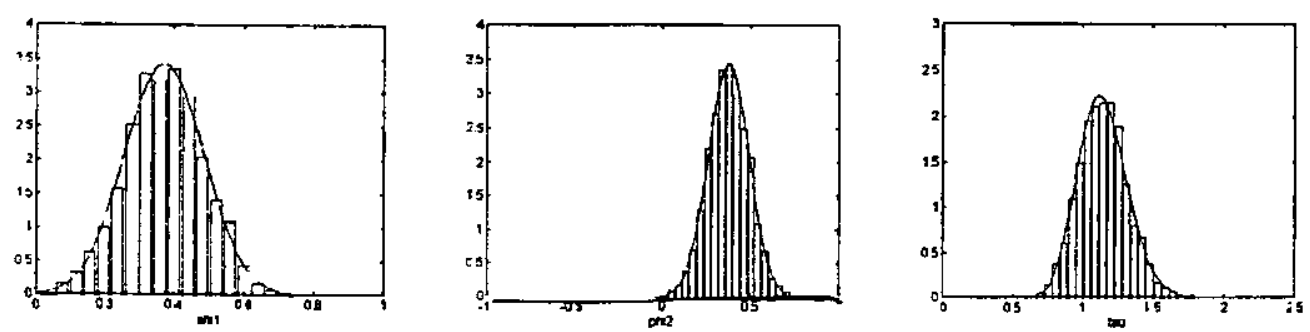

Figura 9. Densidades a Posteriori Marginais para a série de Furnas considerando a priori Normal-Gama para os meses de Julho a Dezembro respectivamente. 

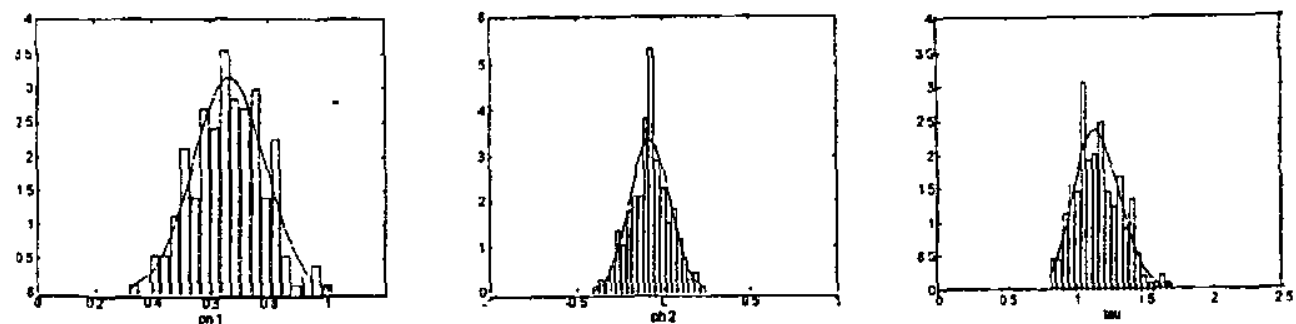

Fevereiro
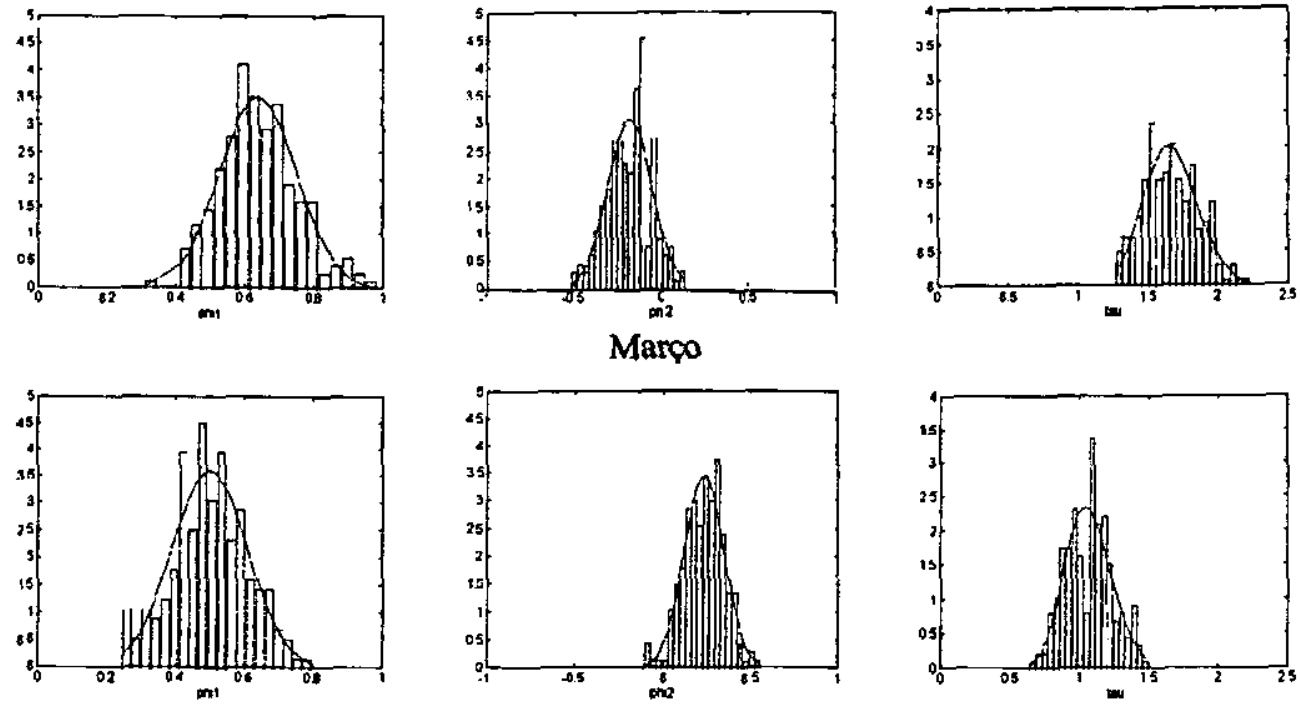

Abril
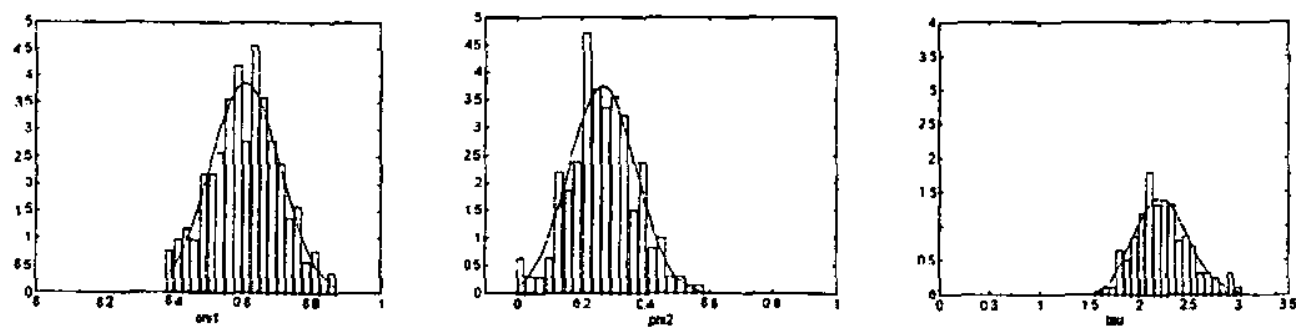

Maio
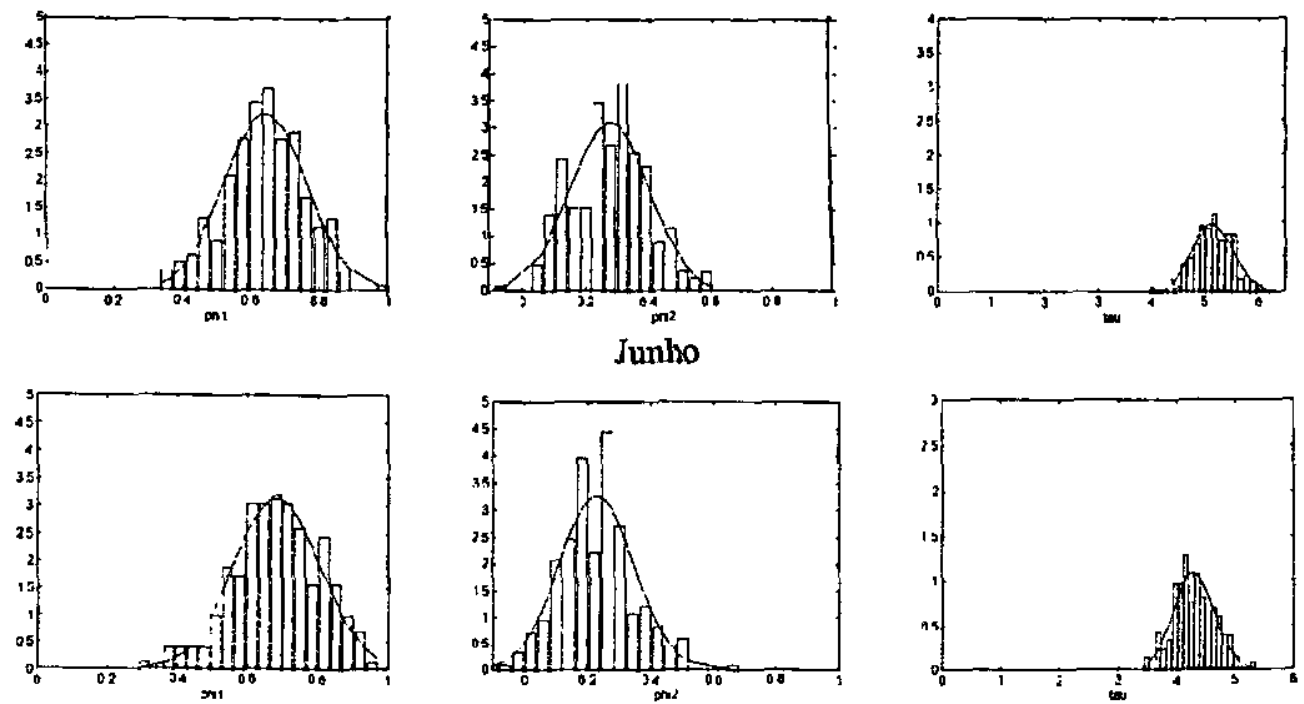

Figura 10. Densidades a Posteriori Marginais para a série de Furnas considerando a priori t-Student e Gama para os meses de Janeiro a Junho respectivamente. 


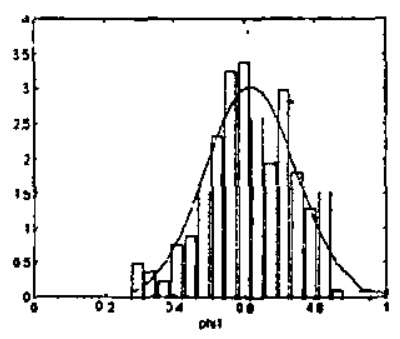

\section{Julho}
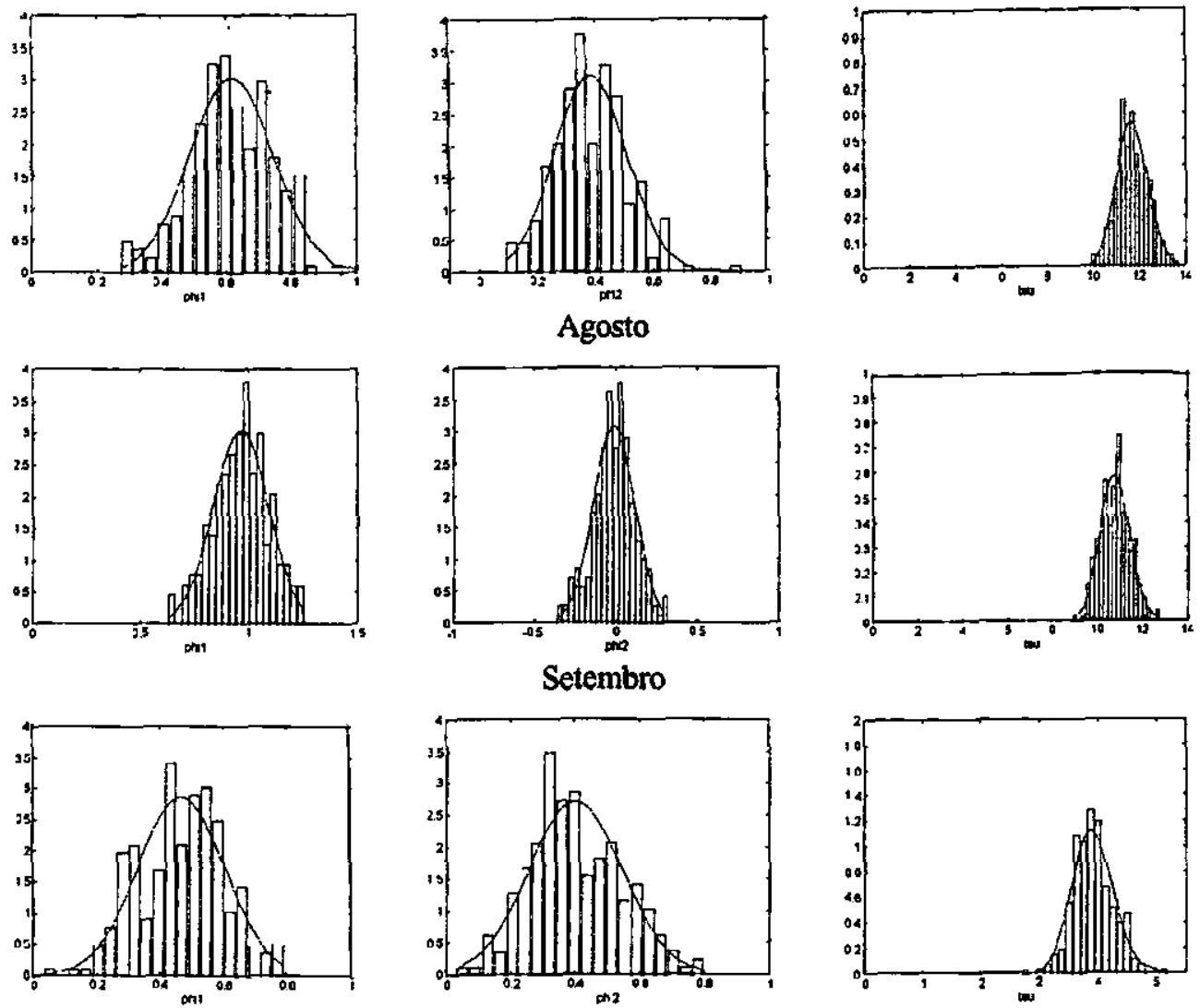

Outubro
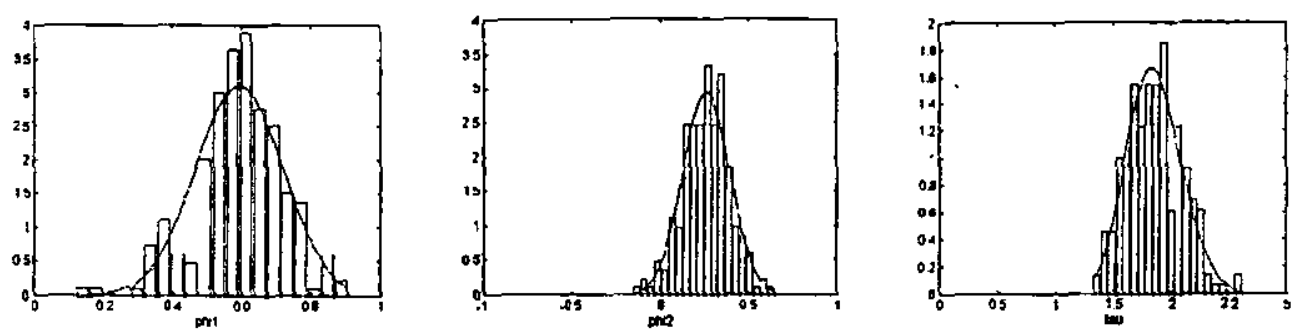

Novembro
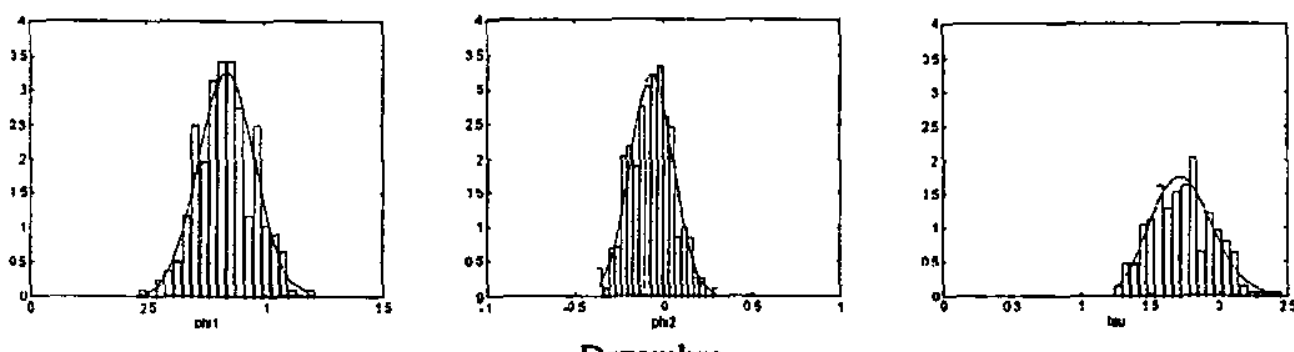

Dezembro
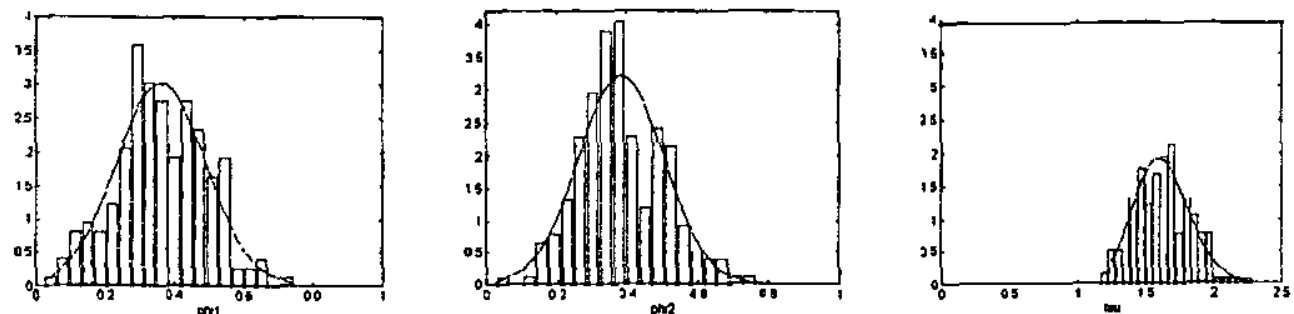

Figura 11. Densidades a Posteriori Marginais para a série de Furnas considerando a priori t-Student e Gama para os meses de Julho a Dezembro respectivamente. 
No caso particular da priori $t$-Student, onde utilizamos o algoritmo de MetropolisHastings foram geradas amostras de tamanho 6000, desprezamos 3000 e selecionamos para estimação uma amostra de tamanho 200 tomando-se um valor a cada 15 gerado na última sequência de 3000 valores gerados. Esse procedimento mostrou-se necessário para evitar a correlação entre os valores gerados devido a rejeição de novos valores.

A Figura 12 a seguir mostra a correlação entre os valores gerados quando não se adota seleção espaçada e a Figura 13 mostra a correlação entre os valores gerados quando se adota seleção espaçada, para os dados da série de Furnas.

Mostramos apenas as correlações do mês de Fevereiro, pois notamos que para os demais meses as correlações são similares.

As Figura 14, 15 e 16 respectivamente mostram a convergência dos dados da série, partindo-se de uma condição inicial arbitrária, mostrando de forma gráfica a convergência do algoritmo MCMC.
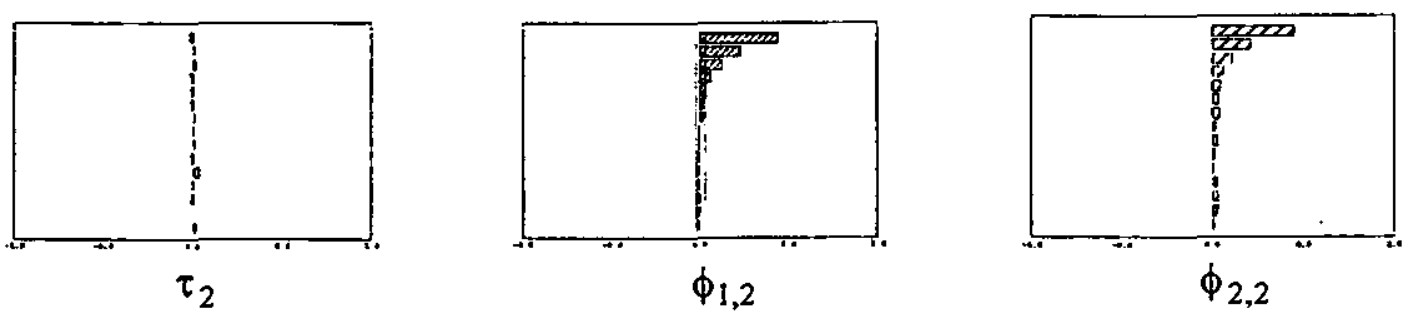

Figura 12. Correlação da amostra gerada pelo amostrador de Gibbs com Metropolis de $\tau_{\mathrm{m}}, \phi_{1, \mathrm{~m}}$ e $\phi_{2, \mathrm{~m}}$ para o mês de Fevereiro considerando os 3000 pontos finais da seqüência.

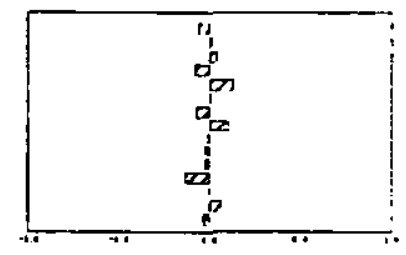

$\tau_{2}$

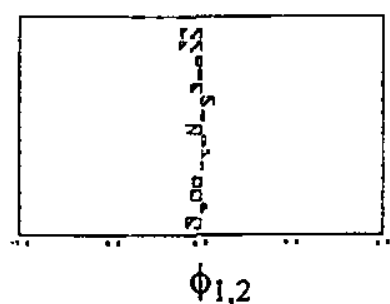

$\phi_{1,2}$

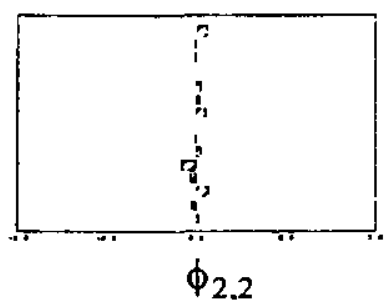

$\phi_{2.2}$

Figura 13. Correlação da amostra de $\tau_{m}, \phi_{1, m}$ e $\phi_{2, m}$ para o mês de Fevereiro, desprezando os 3000 valores iniciais da última seqüência e selecionando-se os 200 pontos restantes de $15 \mathrm{em} 15$. 

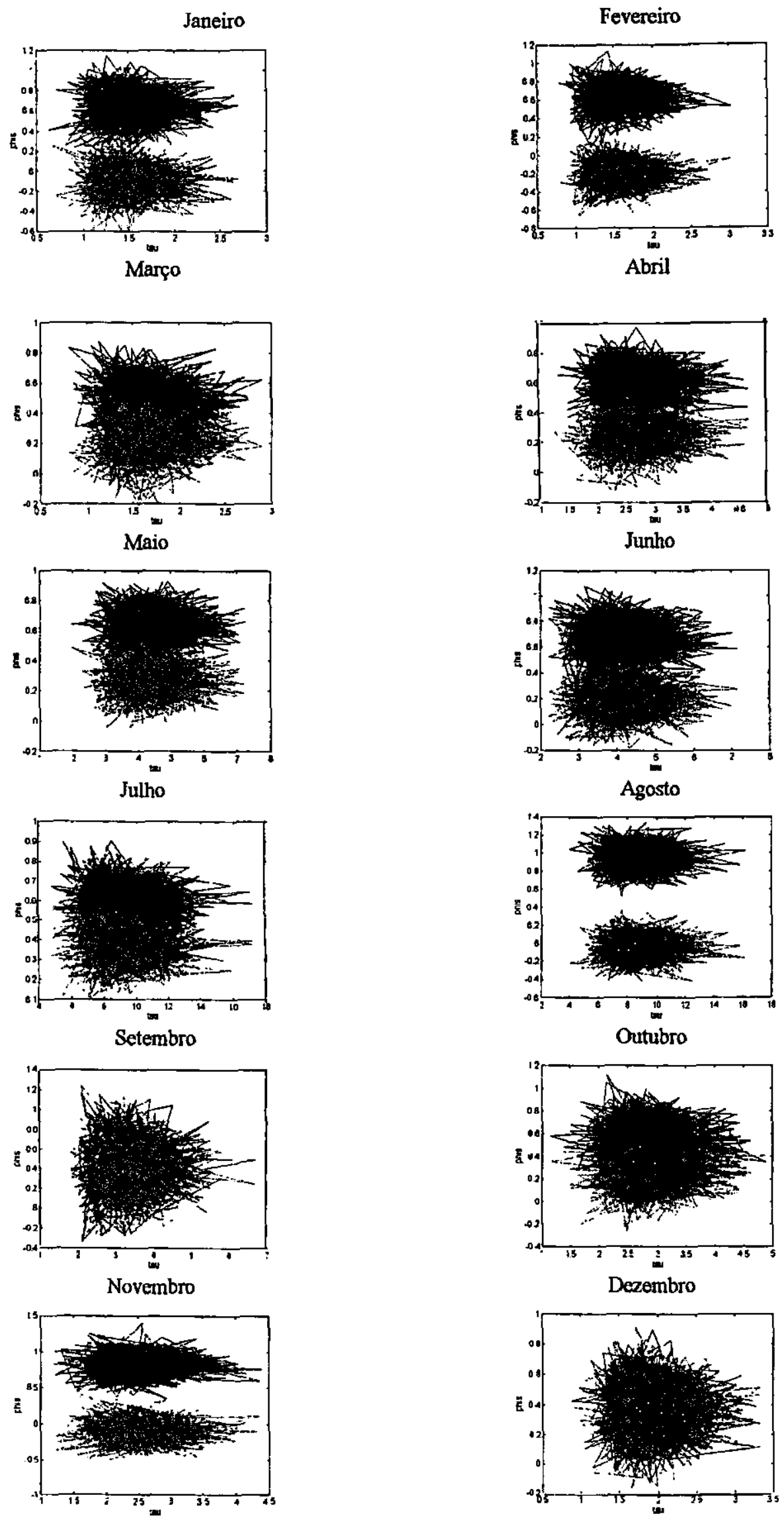

Figura 14. Convergência para a série de Furnas considerando a priori Não - Informativa para os meses de Janeiro a Dezembro respectivamente. 


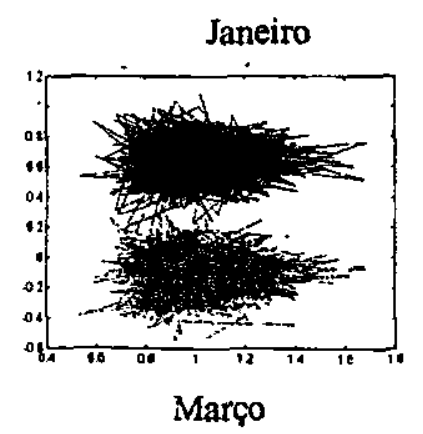

\section{Fevereiro}
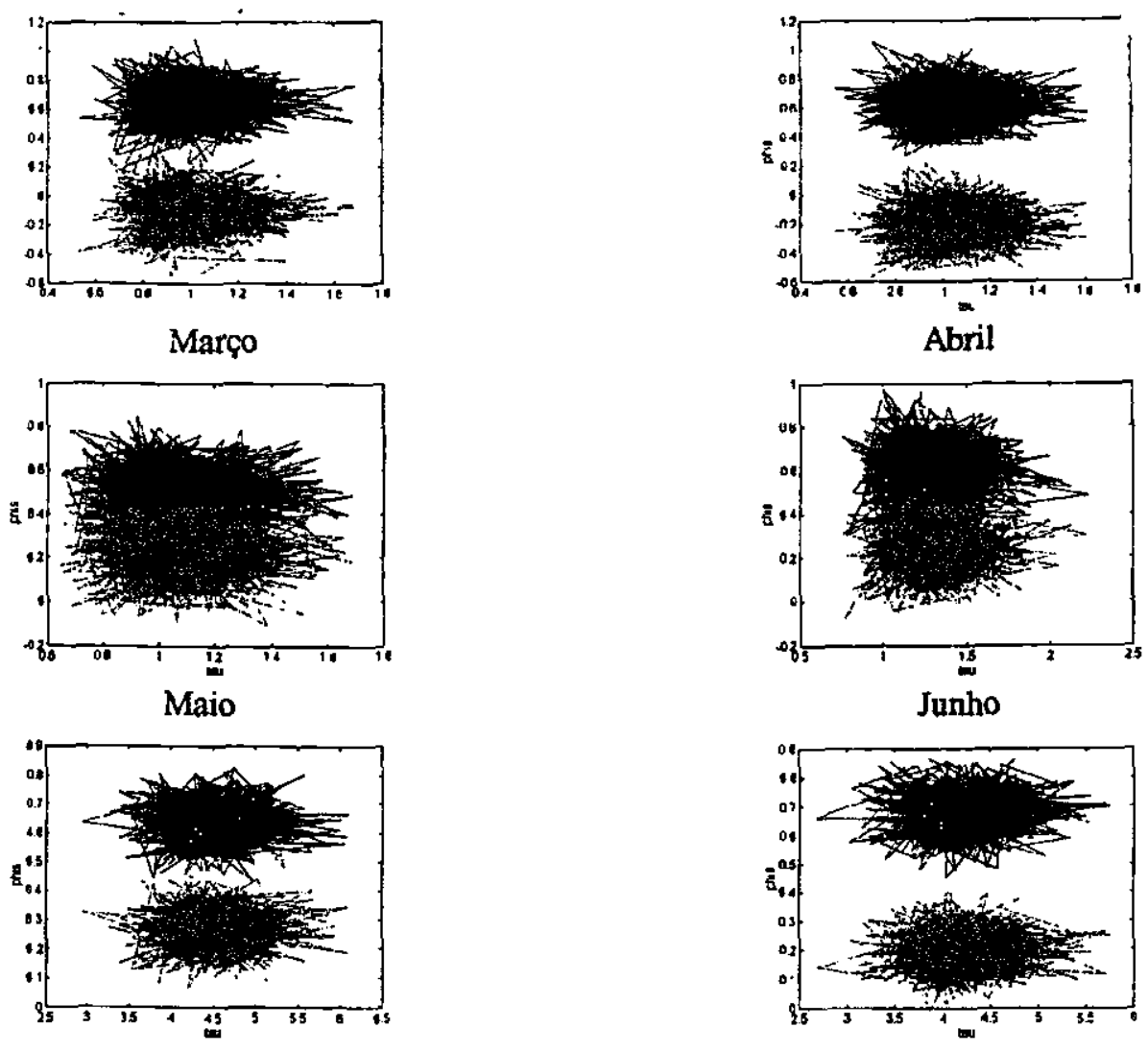

Julho

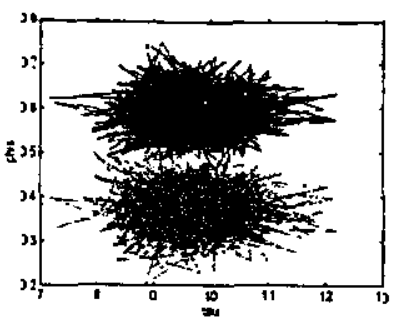

Agosto

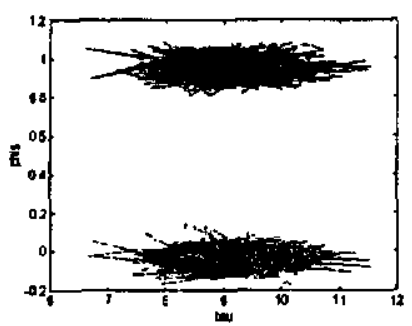

Outubro
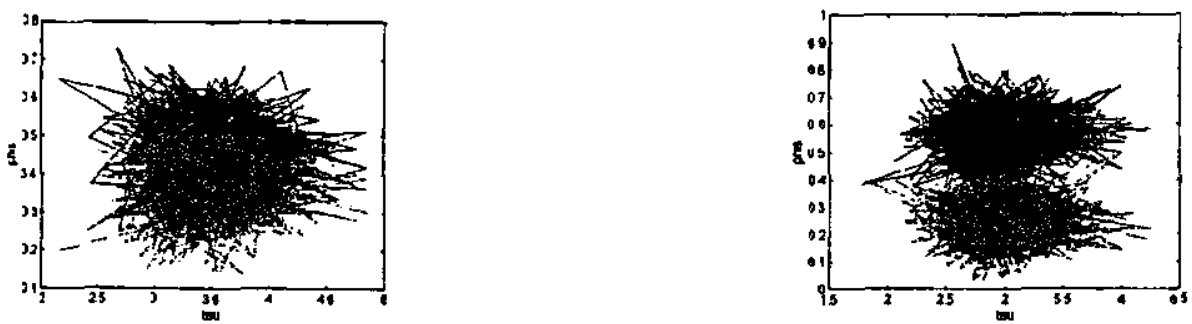

Novembro
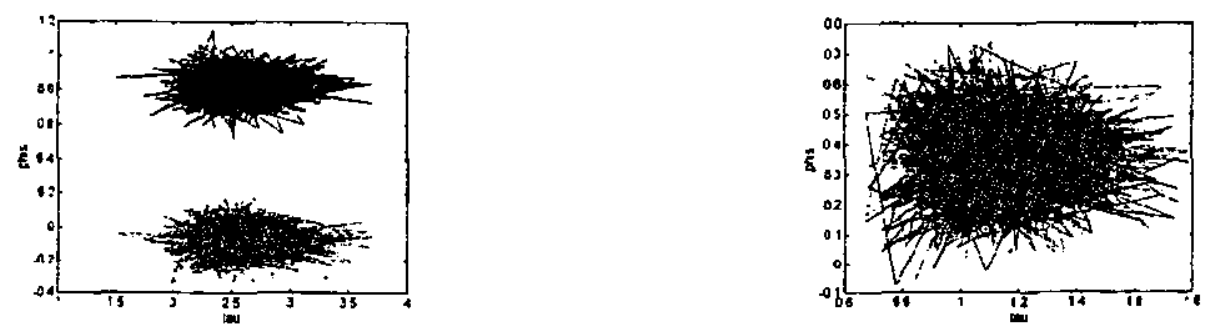

Figura 15. Convergência para a série de Furnas considerando a priori Normal-Gama para os meses de Janeiro a Dezembro respectivamente. 
Janeiro
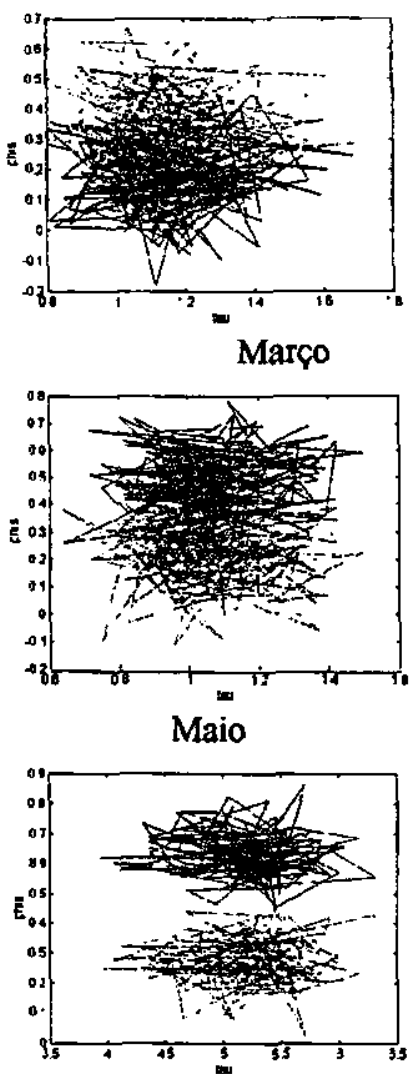

Julho

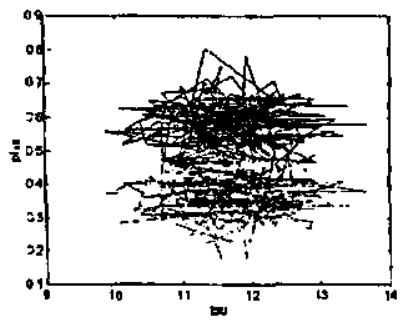

Setembro

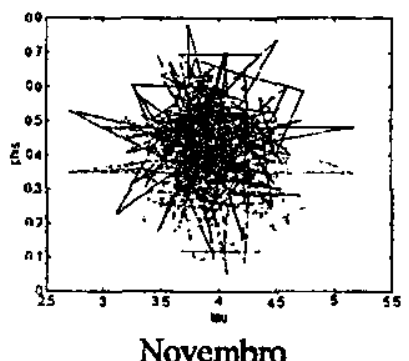

Novembro

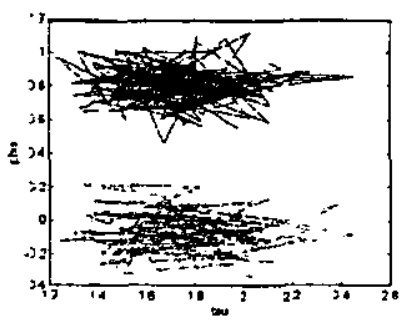

Fevereiro

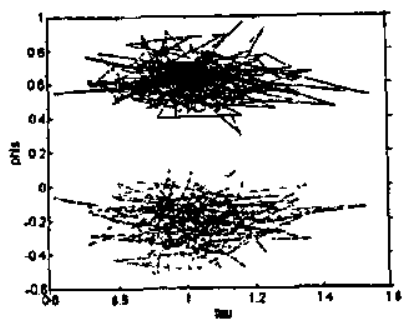

Abril

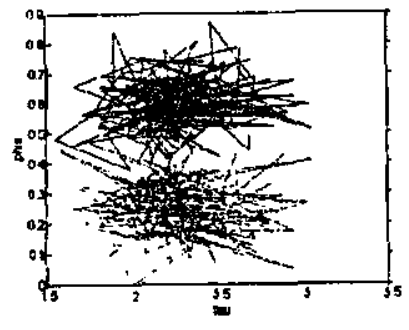

Junho

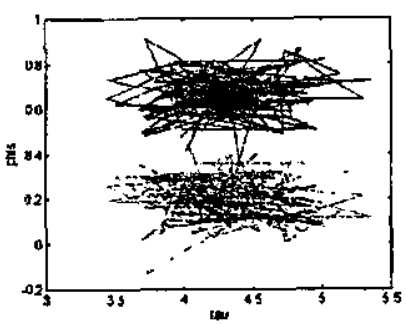

Agosto

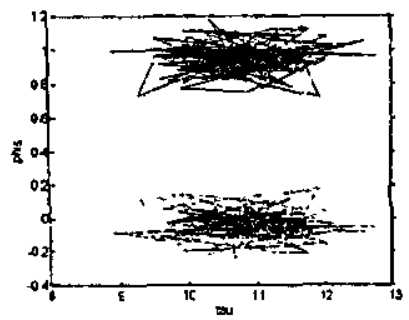

Outubro

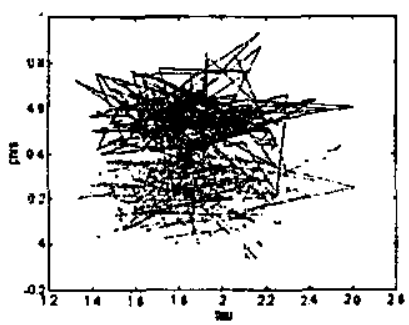

Dezembro

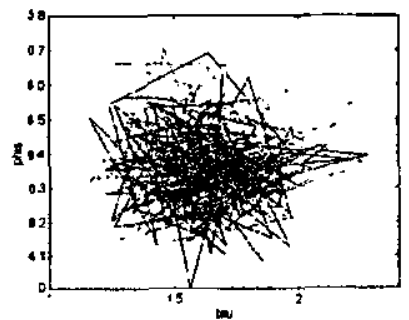

Figura 16. Convergência para a série de Furnas considerando a priori t-Student e Gama para os meses de Janeiro a Dezembro respectivamente. 
As Tabelas 8 a 19, apresentam os estimadores dos parâmetros para cada mês $m$, considerando as três densidades a priori analisadas neste trabalho. Como notação usaremos: EMV para os estimadores de máxima verossimilhança, PNI(.) corresponde ao estimador quando adotamos priori Não-Informativa, PNG(.) corresponde ao estimador quando adotamos priori conjugada Normal-Gama, PTSG(.) corresponde ao estimador quando adotamos o produto das densidades a priori $t$ Student e gama, EX é o estimador exato obtido pelas expressões do valor esperado da densidade a posteriori, GS é o estimador obtido por simulação usando-se o amostrador de Gibbs, MH é o estimador obtido por simulação usando-se o algoritmo de Metropolois-Hastings, DP é o desvio-padrão do estimador, $\mathbf{R}$ é critério de convergência proposto por Gelman e Rubin (1992) ver apêndice, quando os estimadores são obtidos por simulação. Este critério assegura a convergência quando $\mathrm{R}<1,1$.

Em particular quando trabalhamos com o produto das priori $t$-Student e Gama, a posteriori dos parâmetros não tem uma distribuição conhecida, portanto o intervalo de credibilidade é calculado empiricamente dos dados simulados e os resultados são apresentados na Tabela 20. 
Tabela 8. Valores Estimados dos parâmetros para o mês de Janeiro

\begin{tabular}{l|rrrrrrrrr}
\hline & $\tau_{m}$ & $D P$ & $R$ & $\phi_{1, m}$ & $D P$ & $R$ & $\phi_{2, m}$ & $D P$ & $R$ \\
\hline EMV & 1,5149 & 0,2813 & & 0,6535 & 0,1418 & & $-0,0865$ & 0,1411 & \\
PNI(EX) & 1,5149 & 0,2813 & & 0,6535 & 0,1349 & & $-0,0865$ & 0,1342 & \\
PNI(GS) & 1,5178 & 0,2883 & 1,0027 & 0,6535 & 0,1422 & 1,0001 & $-0,0867$ & 0,1468 & 1,0019 \\
PNG(EX) & 1,2815 & 0,1736 & & 0,6553 & 0,1193 & & $-0,0902$ & 0,1190 & \\
PNG(GS) & 1,2810 & 0,1742 & 1,0014 & 0,6534 & 0,1226 & 1,0000 & $-0,0905$ & 0,1176 & 0,9997 \\
PTSG(MH) & 1,1514 & 0,1706 & 1,0288 & 0,6503 & 0,1239 & 1,0122 & $-0,0870$ & 0,1300 & 0,9971 \\
\hline
\end{tabular}

Tabela 9. Valores Estimados dos parâmetros para o mês de Fevereiro

\begin{tabular}{l|ccccccccc}
\hline & $\tau_{m}$ & $D P$ & $R$ & $\phi_{l, m}$ & $D P$ & $R$ & $\phi_{2, m}$ & $D P$ & $R$ \\
\hline EMV & 1,5796 & 0,2933 & & 0,6386 & 0,1282 & & $-0,1870$ & 0,1302 & \\
PNI(EX) & 1,5796 & 0,2933 & & 0,6386 & 0,1219 & & $-0,1870$ & 0,1238 & \\
PNI(GS) & 1,5799 & 0,2967 & 0,9997 & 0,6404 & 0,1293 & 0,9999 & $-0,1861$ & 0,1331 & 1,0005 \\
PNG(EX) & 1,5043 & 0,1967 & & 0,6228 & 0,1140 & & $-0,1790$ & 0,1149 & 1,0011 \\
PNG(GS) & 1,5041 & 0,1974 & 1,0011 & 0,6274 & 0,1138 & 1,0079 & $-0,1804$ & 0,1183 & 1,0015 \\
PTSG(MH) & 1,6617 & 0,1983 & 1,0121 & 0,6455 & 0,1169 & 0,9978 & $-0,1983$ & 0,1082 & 0,9982 \\
\hline
\end{tabular}

Tabela 10. Valores Estimados dos parâmetros para o mês de Março

\begin{tabular}{l|ccccccccc}
\hline & $\tau_{m}$ & $D P$ & $R$ & $\phi_{1, m}$ & $D P$ & $R$ & $\phi_{2, m}$ & $D P$ & $R$ \\
\hline EMV & 1,6838 & 0,3127 & & 0,4903 & 0,1171 & & 0,2366 & 0,1171 & \\
PNI(EX) & 1,6838 & 0,3127 & & 0,4903 & 0,1114 & & 0,2366 & 0,1114 & \\
PNI(GS) & 1,6912 & 0,3069 & 1,0002 & 0,4906 & 0,1166 & 1,0024 & 0,2377 & 0,1219 & 1,0021 \\
PNG(EX) & 1,6656 & 0,2115 & & 0,4950 & 0,1122 & & 0,2357 & 0,1109 & \\
PNG(GS) & 1,6635 & 0,2117 & 1,0000 & 0,4969 & 0,1107 & 1,0003 & 0,2328 & 0,1107 & 1,0005 \\
PTSG(MH) & 1,6912 & 0,3069 & 1,0191 & 0,4882 & 0,1184 & 1,0541 & 0,2287 & 0,1202 & 1,0181 \\
\hline
\end{tabular}

Tabela 11. Valores Estimados dos parâmetros para o mês de Abril

\begin{tabular}{l|ccccccccc}
\hline & $\tau_{m}$ & $D P$ & $R$ & $\phi_{1, m}$ & $D P$ & $R$ & $\phi_{2, m}$ & $D P$ & $R$ \\
\hline EMV & 2,7064 & 0,5026 & & 0,6107 & 0,1001 & & 0,2614 & 0,1001 & \\
PNI(EX) & 2,7064 & 0,5026 & & 0,6107 & 0,0952 & & 0,2614 & 0,0952 & \\
PNI(GS) & 2,7088 & 0,5082 & 1,0003 & 0,6128 & 0,1003 & 1,0004 & 0,2571 & 0,1026 & 1,0013 \\
PNG(EX) & 2,7674 & 0,3002 & & 0,6110 & 0,1036 & & 0,2621 & 0,1037 & \\
PNG(GS) & 2,7659 & 0,3017 & 0,9996 & 0,6070 & 0,1040 & 1,0001 & 0,2621 & 0,1037 & 0,9997 \\
PTSG(MH) & 2,2290 & 0,2842 & 1,0191 & 0,6051 & 0,0885 & 1,0541 & 0,2656 & 0,0886 & 1,0191 \\
\hline
\end{tabular}


Tabela 12. Valores Estimados dos parâmetros para o mês de Maio

\begin{tabular}{l|ccccccccc}
\hline & $\tau_{m}$ & $D P$ & $R$ & $\phi_{1, m}$ & $D P$ & $R$ & $\phi_{2, m}$ & $D P$ & $R$ \\
\hline EMV & 4,3895 & 0,8151 & & 0,6448 & 0,0975 & & 0,2823 & 0,0975 & \\
PNI(EX) & 4,3895 & 0,8151 & - & 0,6448 & 0,0927 & & 0,2823 & 0,0927 & \\
PNI(GS) & 4,3993 & 0,8087 & 1,0027 & 0,6449 & 0,0991 & 1,0003 & 0,2820 & 0,1007 & 1,0008 \\
PNG(EX) & 4,5101 & 0,4117 & & 0,6472 & 0,1027 & & 0,2825 & 0,1025 & \\
PNG(GS) & 4,5062 & 0,4133 & 0,9999 & 0,6500 & 0,1006 & 0,9998 & 0,2790 & 0,1025 & 1,0015 \\
PTSG(MH) & 5,1328 & 0,4002 & 0,9990 & 0,6444 & 0,1276 & 0,9993 & 0,2796 & 0,1257 & 0,9995 \\
\hline
\end{tabular}

Tabela 13. Valores Estimados dos parâmetros para o mês de Junho

\begin{tabular}{l|ccccccccc}
\hline & $\tau_{m}$ & $D P$ & $R$ & $\phi_{1, m}$ & $D P$ & $R$ & $\phi_{2, m}$ & $D P$ & $R$ \\
\hline EMV & 4,1510 & 0,7708 & & 0,6867 & 0,1262 & & 0,2092 & 0,1262 & \\
PNI(EX) & 4,1510 & 0,7708 & & 0,6867 & 0,1200 & & 0,2092 & 0,1200 & \\
PNI(GS) & 4,1473 & 0,7733 & 1,0007 & 0,6844 & 0,1286 & 1,0014 & 0,2128 & 0,1280 & 0,9998 \\
PNG(EX) & 4,2244 & 0,3956 & & 0,6890 & 0,1081 & & 0,2039 & 0,1084 & \\
PNG(GS) & 4,2207 & 0,3964 & 0,9997 & 0,6872 & 0,2015 & 1,0010 & 0,2015 & 0,1074 & 0,9999 \\
PTSG(MH) & 4,2966 & 0,3649 & 0,9965 & 0,6890 & 0,1243 & 1,0011 & 0,1974 & 0,1221 & 1,0141 \\
\hline
\end{tabular}

Tabela 14. Valores Estimados dos parâmetros para o mês de Julho

\begin{tabular}{l|ccccccccc}
\hline & $\tau_{m}$ & $D P$ & $R$ & $\phi_{l, m}$ & $D P$ & $R$ & $\phi_{2, m}$ & $D P$ & $R$ \\
\hline EMV & 4,1510 & 0,7708 & & 0,6867 & 0,1262 & & 0,2092 & 0,1262 & \\
PNI(EX) & 4,1510 & 0,7708 & & 0,6867 & 0,1200 & & 0,2092 & 0,1200 & \\
PNI(GS) & 4,1473 & 0,7733 & 1,0007 & 0,6844 & 0,1286 & 1,0014 & 0,2128 & 0,1280 & 0,9998 \\
PNG(EX) & 9,7028 & 0,6483 & & 0,6021 & 0,1005 & & 0,3760 & 0,1003 & \\
PNG(GS) & 9,6896 & 0,6503 & 1,0002 & 0,6035 & 0,1016 & 1,0000 & 0,3746 & 0,0988 & 1,0011 \\
PTSG(MH) & 11,649 & 0,7031 & 1,0026 & 0,5973 & 0,1172 & 1,0003 & 0,3801 & 0,1276 & 1,0119 \\
\hline
\end{tabular}

Tabela 15. Valores Estimados dos parâmetros para o mês de Agosto

\begin{tabular}{l|ccccccccc}
\hline & $\tau_{m}$ & $D P$ & $R$ & $\phi_{1, m}$ & $D P$ & $R$ & $\phi_{2, m}$ & $D P$ & $R$ \\
\hline EMV & 8,9097 & 1,6545 & & 0,9667 & 0,1167 & & $-0,0254$ & 0,1167 & \\
PNI(EX) & 8,9097 & 1,6445 & & 0,9667 & 0,1109 & & $-0,0254$ & 0,1109 & \\
PNI(GS) & $\mathbf{8 , 9 2 4 4}$ & 1,6787 & 1,0025 & 0,9666 & 0,1193 & 1,0009 & $-0,0253$ & 0,1204 & 1,0003 \\
PNG(EX) & $\mathbf{9 , 1 1 6 4}$ & 0,6261 & & 0,9639 & 0,1044 & & $-0,0254$ & 0,0446 & \\
PNG(GS) & 9,1051 & 0,6296 & 0,9997 & 0,9636 & 0,1031 & 0,9997 & $-0,0253$ & 0,0444 & 1,0001 \\
PTSG(MH) & 10,729 & 0,6787 & 0,9989 & 0,9584 & 0,1341 & 1,0046 & $-0,0157$ & 0,0769 & 1,0046 \\
\hline
\end{tabular}


Tabela 16. Valores Estimados dos parâmetros para o mês de Setembro

\begin{tabular}{l|ccccccccc}
\hline & $\tau_{m}$ & $D P$ & $R$ & $\phi_{1, m}$ & $D P$ & $R$ & $\phi_{2, m}$ & $D P$ & $R$ \\
\hline EMV & 3,4161 & 0,6344 & & 0,4710 & 0,2120 & & 0,3850 & 0,2120 & \\
PNI(EX) & 3,4161 & 0,6344 & - & 0,4710 & 0,2016 & & 0,3850 & 0,2016 & \\
PNI(GS) & 3,4159 & 0,6292 & 0,9997 & 0,4712 & 0,2123 & 0,9999 & 0,3866 & 0,2118 & 0,9997 \\
PNG(EX) & 3,5101 & 0,3510 & & 0,4710 & 0,1167 & & 0,3850 & 0,1169 & \\
PNG(GS) & 3,5076 & 0,3534 & 1,0002 & 0,4698 & 0,1201 & 1,0019 & 0,3846 & 0,1162 & 1,0015 \\
PTSG(MH) & 3,8968 & 0,3555 & 1,0053 & 0,4796 & 0,1380 & 1,0215 & 0,3865 & 0,1504 & 0,9964 \\
\hline
\end{tabular}

Tabela 17. Valores Estimados dos parâmetros para o mês de Outubro

\begin{tabular}{l|ccccccccc}
\hline & $\tau_{m}$ & $D P$ & $R$ & $\phi_{l, m}$ & $D P$ & $R$ & $\phi_{2, m}$ & $D P$ & $R$ \\
\hline EMV & 2,9068 & 0,5398 & & 0,5806 & 0,1385 & & 0,2635 & 0,1385 & \\
PNI(EX) & 2,9068 & 0,5398 & & 0,5806 & 0,1317 & & 0,2635 & 0,1385 & \\
PNI(GS) & 2,9035 & 0,5514 & 1,0003 & 0,5821 & 0,1396 & 1,0019 & 0,2606 & 0,1410 & 1,0041 \\
PNG(EX) & 3,0023 & 0,3165 & & 0,5850 & 0,1122 & & 0,2580 & 0,1123 & \\
PNG(GS) & 2,9977 & 0,3166 & 0,9995 & 0,5875 & 0,1120 & 0,9999 & 0,2574 & 0,1105 & 0,9995 \\
PTSG(MH) & 1,8538 & 0,2410 & 1,0139 & 0,5665 & 0,1298 & 1,0257 & 0,2724 & 0,1212 & 1,0291 \\
\hline
\end{tabular}

Tabela 18. Valores Estimados dos parâmetros para o mês de Novembro

\begin{tabular}{l|ccccccccc}
\hline & $\tau_{m}$ & $D P$ & $R$ & $\phi_{1, m}$ & $D P$ & $R$ & $\phi_{2, m}$ & $D P$ & $R$ \\
\hline EMV & 2,5208 & 0,4681 & & 0,8329 & 0,1368 & & $-0,0661$ & 0,1368 & \\
PNI(EX) & 2,5208 & 0,4681 & & 0,8329 & 0,1301 & & $-0,0661$ & 0,1301 & \\
PNI(GS) & 2,5283 & 0,4809 & 0,9998 & 0,8333 & 0,1377 & 1,0011 & $-0,0675$ & 0,1394 & 0,9995 \\
PNG(EX) & 2,6028 & 0,2874 & & 0,8181 & 0,1126 & & $-0,0661$ & 0,1125 & \\
PNG(GS) & 2,6010 & 0,2891 & 1,0010 & 0,8241 & 0,1124 & 0,9997 & $-0,0681$ & 0,1111 & 0,9999 \\
PTSG(MH) & 1,7320 & 0,2275 & 1,0035 & 0,8190 & 0,1121 & 1,0107 & $-0,0654$ & 0,1288 & 1,0203 \\
\hline
\end{tabular}

Tabela 19. Valores Estimados dos parâmetros para o mês de Dezembro

\begin{tabular}{l|ccccccccc}
\hline & $\tau_{m}$ & $D P$ & $R$ & $\phi_{1, m}$ & $D P$ & $R$ & $\phi_{2, m}$ & $D P$ & $R$ \\
\hline EMV & 1,9399 & 0,3602 & & 0,3661 & 0,1494 & & 0,3783 & 0,1494 & \\
PNI(EX) & 1,9399 & 0,3602 & & 0,3661 & 0,1421 & & 0,3783 & 0,1421 & \\
PNI(GS) & 1,9339 & 0,3602 & 0,9999 & 0,3721 & 0,1552 & 1,0049 & 0,3740 & 0,1523 & 1,0003 \\
PNG(EX) & 1,9209 & 0,2356 & & 0,3356 & 0,0900 & & 0,3963 & 0,0894 & \\
PNG(GS) & 1,9521 & 0,2421 & 0,9995 & 0,3355 & 0,0912 & 1,0010 & 0,3970 & 0,0892 & 1,0008 \\
PTSG(MH) & 1,6184 & 0,2080 & 1,0069 & 0,3789 & 0,1196 & 0,9956 & 0,3680 & 0,1196 & 1,0058 \\
\hline
\end{tabular}


Tabela 20. Intervalo de Credibilidade para os parâmetros $\tau_{\mathrm{m}}, \phi_{1, \mathrm{~m}}$ e $\phi_{2, \mathrm{~m}}$ (Priori $t$-Student e Gama- Janeiro a Dezembro)

\begin{tabular}{l|ccc}
\hline mês $m$ & $\tau_{m}$ & $\phi_{1, m}$ & $\phi_{2, m}$ \\
\hline Janeiro $(m=1)$ & $(0,7144 ; 1,3125)$ & $(0,4072 ; 0,8871)$ & $(-0,3560 ; 0,1381)$ \\
Fevereiro $(m=2)$ & $(0,7389 ; 1,4275)$ & $(0,4027 ; 0,8389)$ & $(-0,4179 ;-0,0170)$ \\
Março $(m=3)$ & $(0,7402 ; 1,4244)$ & $(0,2700 ; 0,7289)$ & $(0,0025 ; 0,4689)$ \\
Abril $(m=4)$ & $(0,9143 ; 1,6925)$ & $(0,3960 ; 0,8166)$ & $(0,0309 ; 0,4910)$ \\
Maio $(m=5)$ & $(1,0735 ; 2,0185)$ & $(0,3656 ; 0,8778)$ & $(0,0256 ; 0,5060)$ \\
Junho $(m=6)$ & $(1,0412 ; 1,9598)$ & $(0,4202 ; 0,9317)$ & $(-0,0278 ; 0,4075)$ \\
Julho $(m=7)$ & $(1,2417 ; 2,3372)$ & $(0,3800 ; 0,8057)$ & $(0,1443 ; 0,6263)$ \\
Agosto $(m=8)$ & $(1,2339 ; 2,3492)$ & $(0,6727 ; 1,2191)$ & $(-0,2512 ; 0,2458)$ \\
Setembro $(m=9)$ & $(0,9658 ; 1,7942)$ & $(0,1964 ; 0,7260)$ & $(0,1108 ; 0,9658)$ \\
Outubro $(m=10)$ & $(0,9496 ; 1,7526)$ & 0,$3106 ; 0,80240$ & $(0,0102 ; 0,5541)$ \\
Novembro $(m=11)$ & $(0,8088 ; 1,6117)$ & $(0,5931 ; 1,0665)$ & $(-0,3259 ; 0,1962)$ \\
Dezembro $(m=12)$ & $(0,8047 ; 1,5611)$ & $(0,1320 ; 0,6497)$ & $(0,1518 ; 0,5789)$ \\
\hline
\end{tabular}

Com uma amostra de tamanho 3000 a probabilidade de que o intervalo calculado a partir dessa amostra contenha o parâmetro é $95 \%$. Observando os intervalos de credibilidade dados na Tabela 20 , podemos dizer que os meses de Janeiro, Fevereiro, Junho, Agosto e Novembro para o parâmetro $\phi_{2, m}$ seus respectivos intervalos contém o valor zero, isto é, estes intervalos não são siginificativamente diferentes de zero, portanto para esses específicos meses podemos desprezar o parâmetro $\phi_{2, m}$, ajustando desta forma modelos de ordem um, o que confere com o BIC.

Nas Figuras 6 a 9 podemos observar que a forma simétrica da posteriori é a forma esperada teoricamente como foi deduzido na seção 3.3 , onde vimos a distribuição conjunta a posteriori para $\phi_{1, m}, \phi_{2, m}$ e $\tau_{m}$ é uma Normal-Gama.

Observamos nas Figuras 10 e 11 que o comportamento das distribuições a posteriori é aquele esperado na seção 3.4, e que com o uso do produto das priori $t$-Student e Gama apesar de não conhecermos a distribuição a posteriori, os his- 
togramas também se apresentam de uma forma simétrica.

Com os resultados obtidos nas Tabelas 8 a 19, observamos que as estimativas pontuais são próximas para os parâmetros $\phi_{1, m}, \phi_{2, m}$ e $\tau_{m}$, considerando o método Clássico e Bayesiano com a densidade a priori Não-Informativa. Observamos que quando usamos as densidades a priori informativas as estimativas a posteriori para o parâmetro $\tau_{m}$ apresenta muita variação ao contrário das estimativas para os parâmetros $\phi_{1, m}$ e $\phi_{2, m}$ que apresentamvalores próximos em todos os casos.

A convergência (em distribuição) dos algoritmos Gibbs Sampling e MetropolisHastings é verificada pelo critério de Gelman e Rubin (1992) Observamos através de $R$ (fator de redução de escala) e pelos gráficos das Figuras 14,15 e 16 que a convergência é alcançada para as três densidades a priori estudadas neste trabalho.

\subsection{Aplicações para o Exemplo 6}

Série com dados reais de vazões médias mensais da barragem de Emborcação. Esta é uma série mensal com dados com 60 anos. $\mathrm{O}$ modelo ajustado para esta série foi um $\operatorname{PAR}(1)$, dado por:

$$
Z_{t(r, m)}=\frac{\tilde{Z}_{t(r, m)}-\widehat{\mu}_{m}}{\widehat{\sigma}_{m}}
$$

onde

$$
Z_{t(r, m)}=\phi_{1, m} Z_{t(r, m)-1}+a_{t(r, m)}
$$

Observamos a seguir os gráficos das funções de autocorrelação (PeFAC) e autocorrelação parcial (PeFACP) mostradas pela Figura 17. 
Através da análise da PeFAC e PeFACP podemos escolher um modelo PAR(1) para a série de Emborcaçao e usando-se o Critério de Informação Bayesiano, BIC, esse modelo mostra-se o mais parcimonioso para todos os meses. Os resultados são apresentados na Tabela 21 .

Tabela 21. Valor do BIC e BIC $\mathrm{m}_{\mathrm{m}}$ para os meses de Janeiro a Dezembro

\begin{tabular}{|c|c|c|c|}
\hline & \multicolumn{3}{|c|}{$\mathrm{BIC}_{\mathrm{m}}$} \\
\hline Meses & $p_{m}=1$ & $p_{m}=2$ & $p_{m}=3$ \\
\hline Janeiro $(m=1)$ & $-0,4319$ & $-0,3637$ & $-0,2954$ \\
\hline Fevereiro $(m=2)$ & $-0,2000$ & $-0,1318$ & $-0,0635$ \\
\hline $\operatorname{Março~}(m=3)$ & $-0,5864$ & $-0,5182$ & $-0,4499$ \\
\hline Abril $(m=4)$ & $-1,5574$ & $-1,1892$ & $-1,4209$ \\
\hline Maio $(m=5)$ & $-1,3310$ & $-1,2628$ & $-1,1945$ \\
\hline Junho $(m=6)$ & $-2,3330$ & $-2,2651$ & $-2,1968$ \\
\hline Julho $(m=7)$ & $-2,2472$ & $-2,1790$ & $-2,1107$ \\
\hline Agosto $(m=8)$ & $-1,2781$ & $-1,2099$ & $-1,1416$ \\
\hline Setembro $(m=9)$ & $-0,1319$ & $-0,0637$ & 0,0040 \\
\hline Outubro $(m=10)$ & $-0,0876$ & $-0,0194$ & 0,0488 \\
\hline Novembro $(m=11)$ & $-0,1806$ & $-0,1124$ & $-0,0441$ \\
\hline Dezembro $(m=12)$ & $-0,0466$ & 0,0224 & 0,0915 \\
\hline $\mathrm{BIC}=\sum_{\mathrm{m}=1}^{12} \mathrm{BIC}_{\mathrm{m}}$ & $-10,4117$ & $-9,5928$ & $-8,7731$ \\
\hline
\end{tabular}



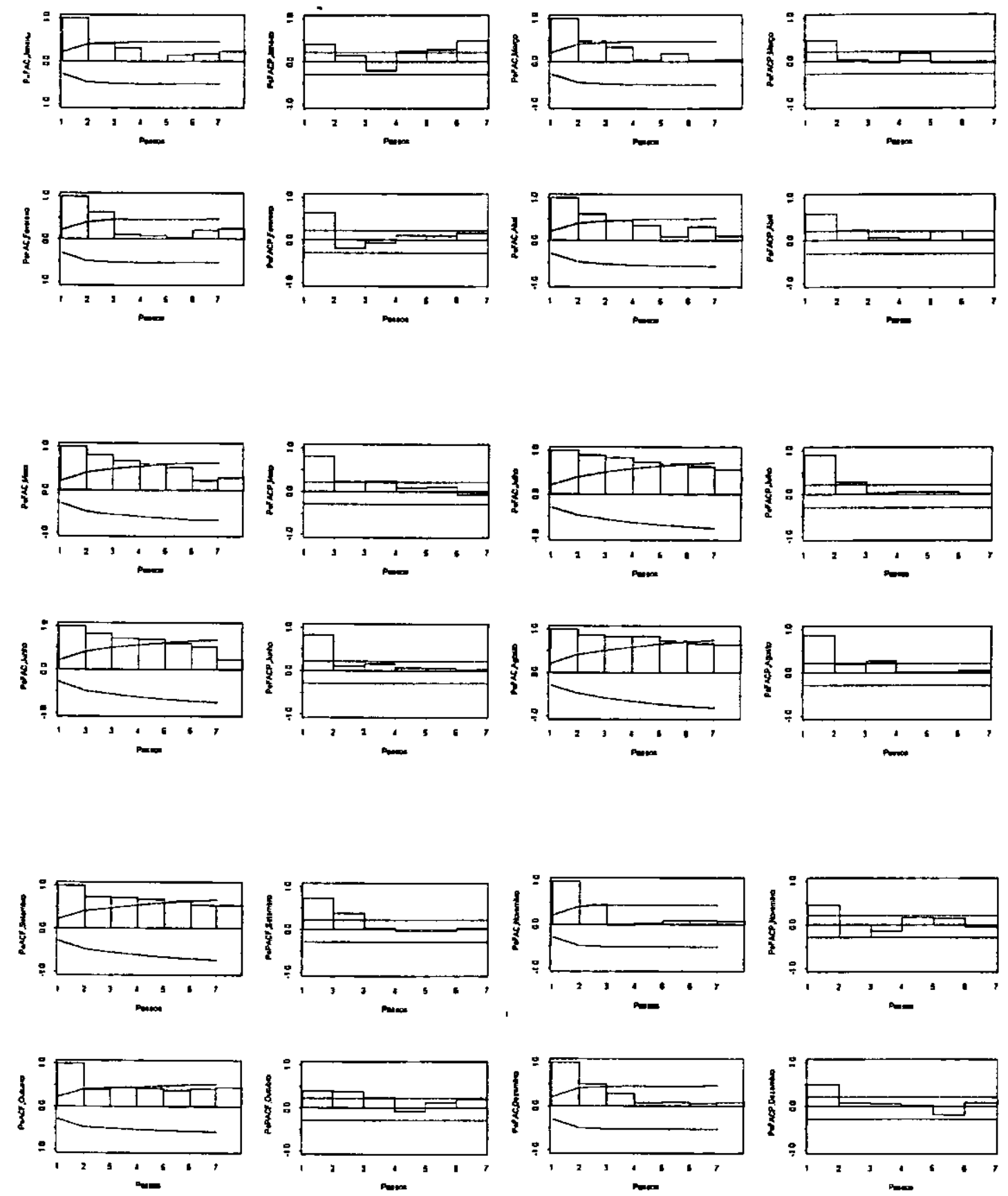

Figura 17. PeFAC e PeFACP para os meses de Janeiro a Dezembro. 
Nesta seção vamos comparar o BIC com o critério de seleção de modelos que utilizam a densidade preditiva ordenada, para o modelo ajustado, os resultados são apresentados na Tabela 22 para seu respectivo mês.

\begin{tabular}{l|l|l|l}
\multicolumn{5}{l}{ Tabela 22. Seleção de Modelos para a série de Emborcação } \\
\hline \multirow{2}{*}{ meses } & $p_{m}=1$ & $p_{m}=2$ & $p_{m}=3$ \\
\cline { 2 - 4 } & $\mathrm{c}(1)$ & $\mathrm{c}(\mathrm{l})$ & $\mathrm{c}(\mathrm{l})$ \\
\hline Janeiro $(\mathrm{m}=1)$ & 0,0000007 & 0,0000008 & 0,00000018 \\
Fevereiro $(\mathrm{m}=2)$ & 0,0004 & 0,0002 & 0,0001 \\
Março $(\mathrm{m}=3)$ & 0,0017 & 0,0017 & 0,0019 \\
Abril $(\mathrm{m}=4)$ & 0,0972 & 0,1423 & 0,1472 \\
Maio $(\mathrm{m}=5)$ & 15,6956 & 26,0920 & 58,4712 \\
Junho $(\mathrm{m}=6)$ & 18,8598 & 18,8390 & 21,3397 \\
Julho $(\mathrm{m}=7)$ & 8,9412 & 2,6791 & 2,0790 \\
Agosto $(\mathrm{m}=8)$ & 99,3060 & 96,3317 & 1,1891 \\
Setembro $(m=9)$ & 0,5826 & 0,5565 & 0,6180 \\
Outubro $(\mathrm{m}=10)$ & 0,0007 & 0,00014 & 0,00016 \\
Novembro $(m=11)$ & 0,000004 & 0,000017 & 0,000017 \\
Dezembro $(m=12)$ & 0,000026 & 0,000022 & 0,000022 \\
\hline
\end{tabular}

Os resultados mostrados nas Tabelas 21 e 22 respectivamente não coincidem para todos os meses é necessário avaliarmos os intervalos de credibilidade.

A Figura 18 mostram os gráficos de $c_{t(r, m)}$ versus $t(r, m), r=1, \ldots, n$ para diferentes modelos da série de Emborcação para os meses de Janeiro a Dezembro. 
Janeiro

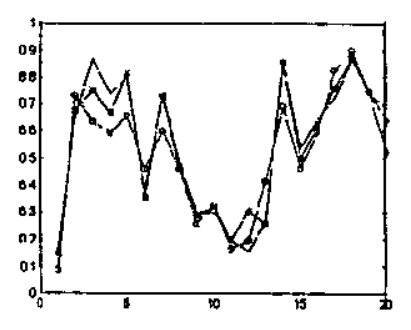

Abril

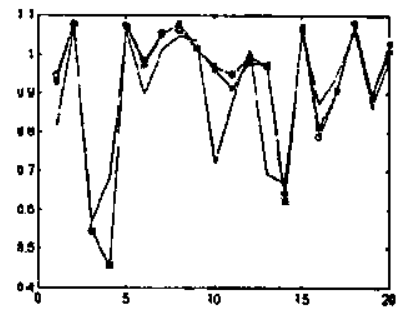

Julho

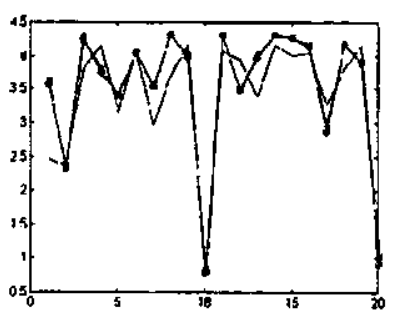

Outubro

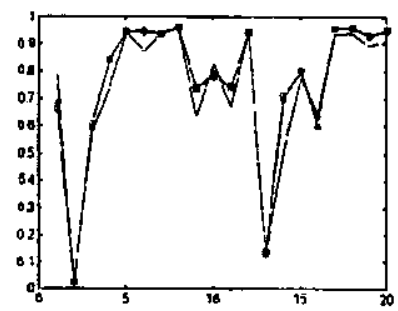

Fevereiro

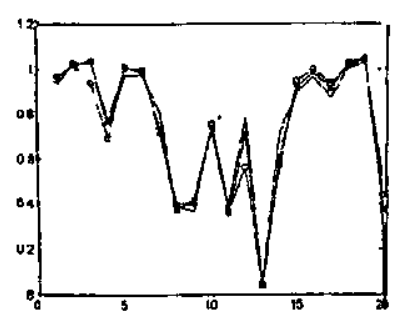

Maio

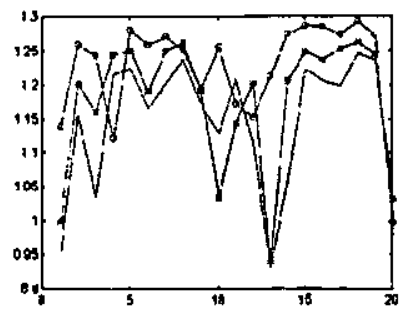

Agosto

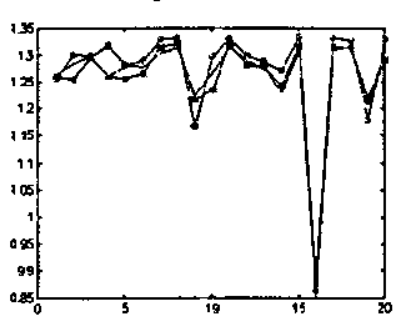

Novembro

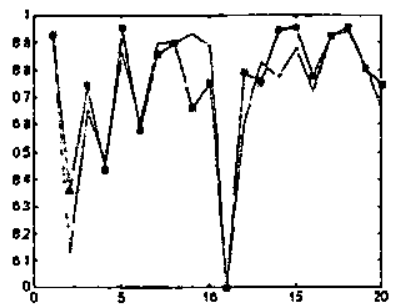

Março

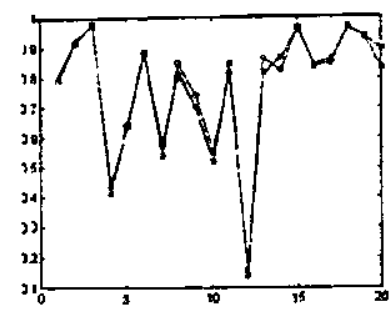

Junho

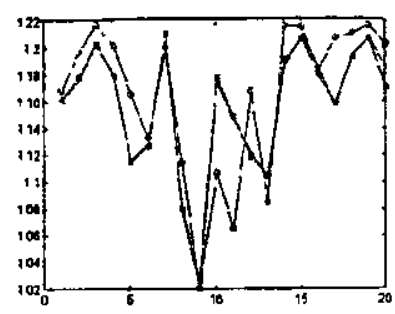

Setembro

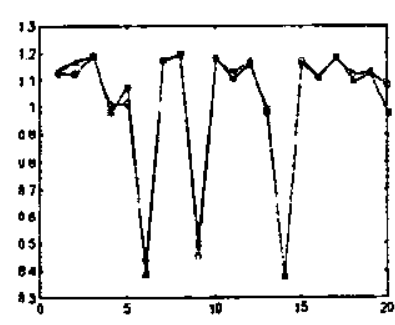

Dezembro

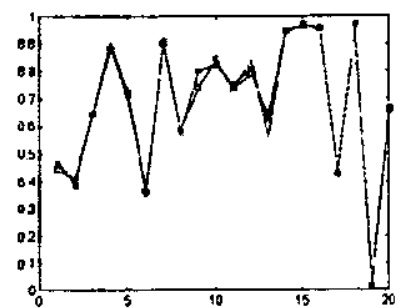

Figura 18. $c_{t(r, m)} \times t(r, m), r=1, \ldots, n$ para diferentes modelos para os meses de Janeiro a Dezembro. 
Como vimos anteriormente com o intuito de analisar o comportamento da densidade aposteriori conjunta, utilizamos os algoritmos Amostrador de Gibbs e Metropolis-Hastings.

Considerando os dados $\mathrm{e}$ as três densidades a priori estudadas neste trabalho, apresentamos os histogramas estimados por simulação para seus respectivos parâmetros para cada mês.

As Densidades a Posteriori Marginais para os parâmetros $\phi_{1, m}$ e $\tau_{m}$, quando consideramos uma priori Não-Informativa para a série de Emborcação são mostrados a seguir pelas Figuras 19 e 20 respectivamente.

Nas Figuras 21 e 22, os histogramas mostram respectivamente as densidades a posteriori marginais para os parâmetros $\phi_{1, m}$ e $\tau_{m}$, quando consideramos uma priori Normal-Gama.

Para o produto das priori $t$-Student e Gama para a série de Emborcação, as Densidades a Posteriori Marginais para os parâmetros $\phi_{1, m}$ e $\tau_{m}$ são exibidos nas Figuras 22 e 23 respectivamente. 
Janeiro
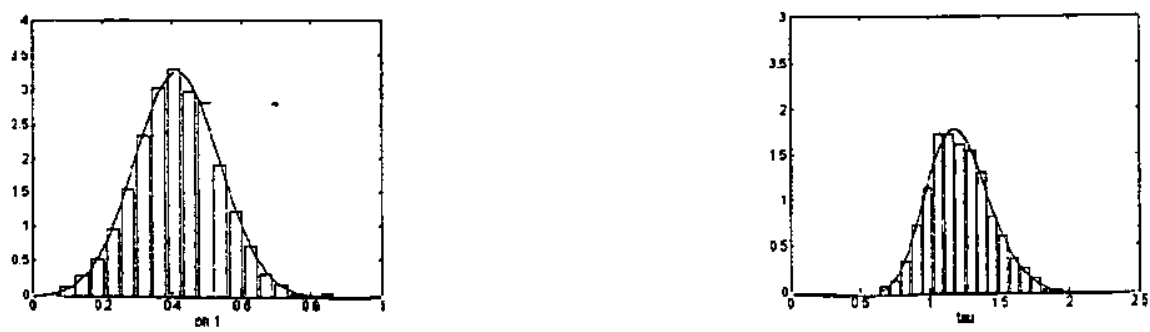

Fevereiro
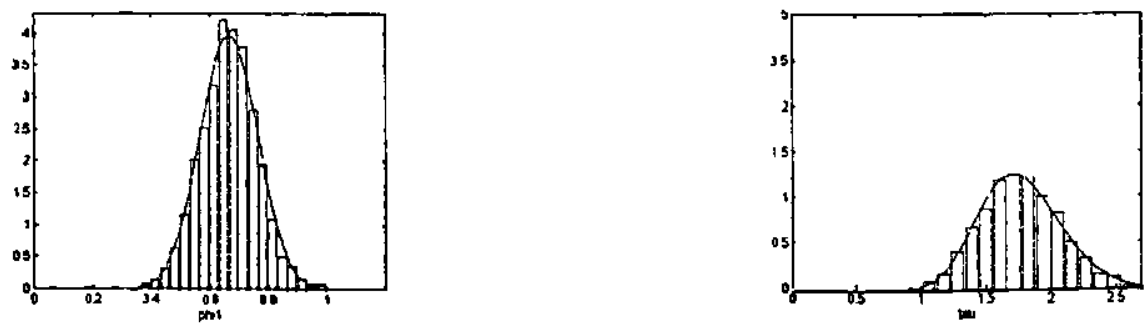

Março
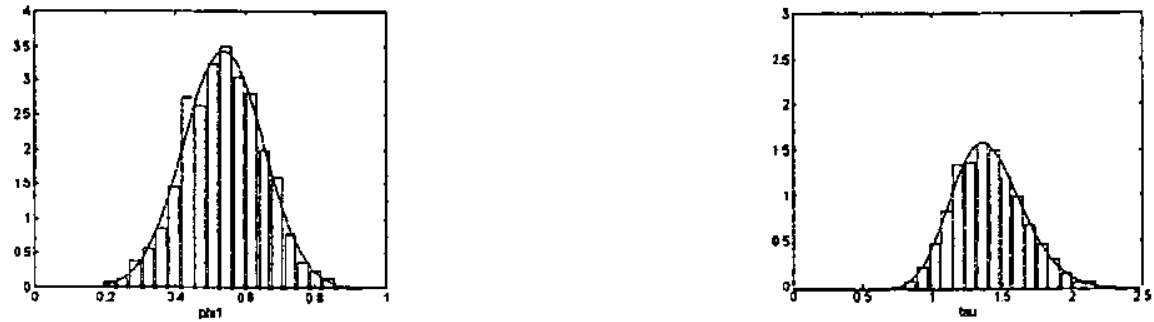

Abril
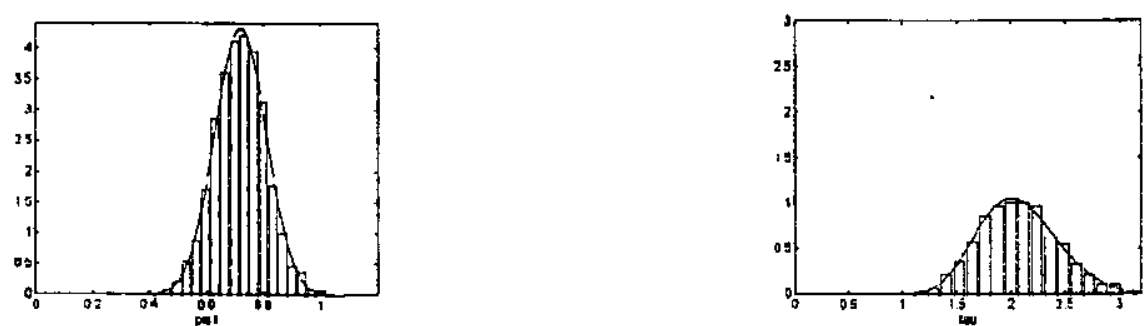

Maio
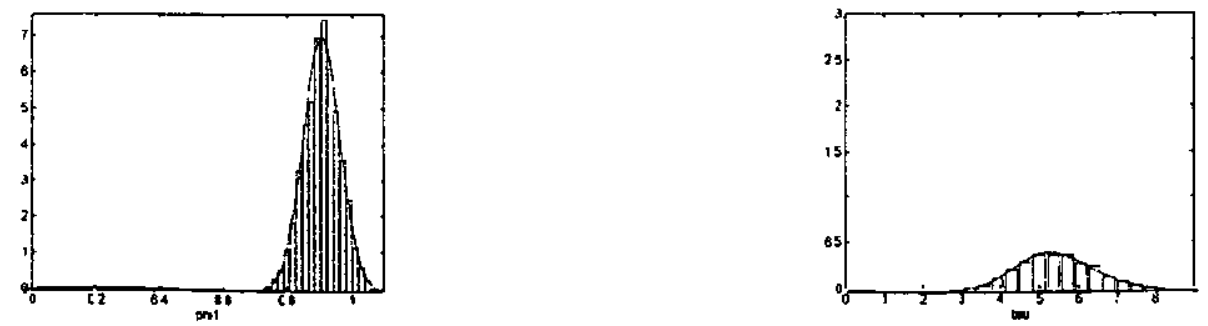

Junho
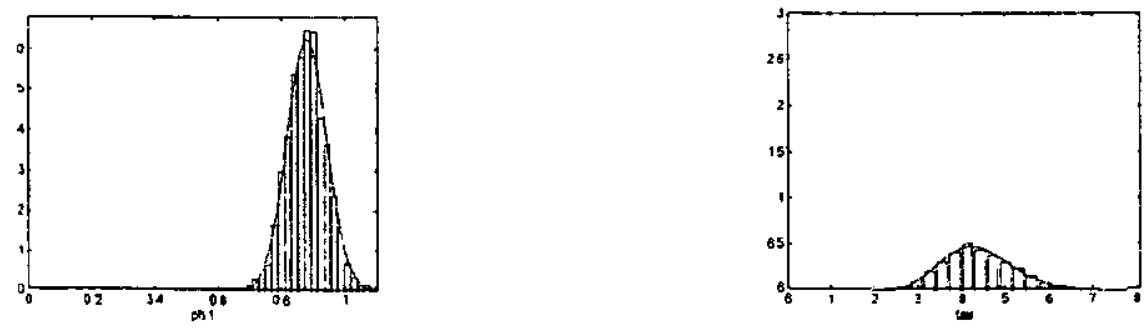

Figura 19. Densidades a Posteriori Marginais para a série de Emborcação considerando a priori Não-Informativa para os meses de Janeiro a Junho respectivamente. 
Julho
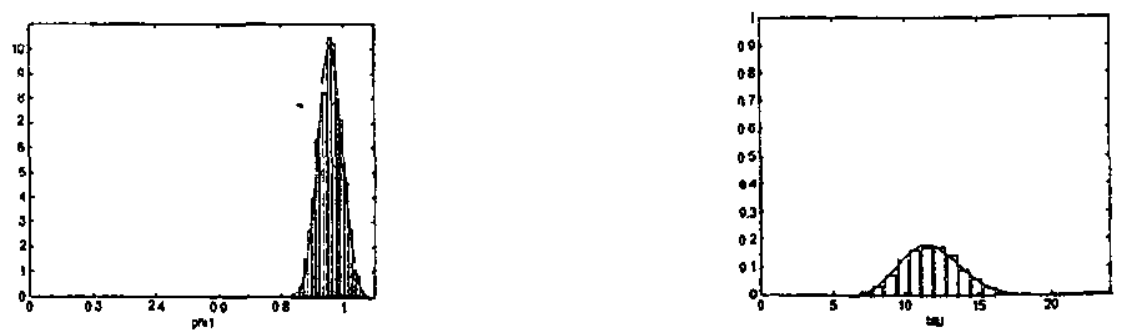

Agosto
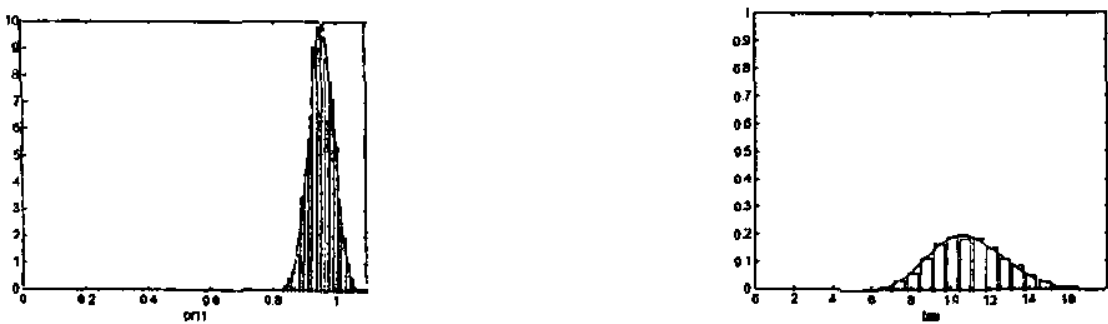

Setembro
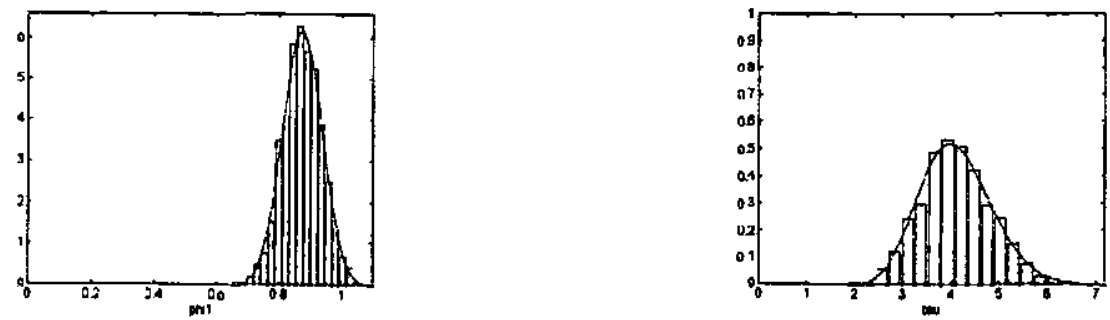

Outubro
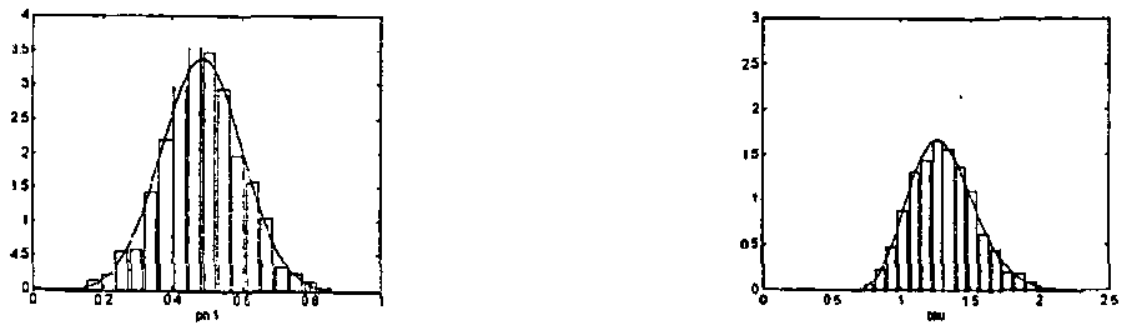

Novembro
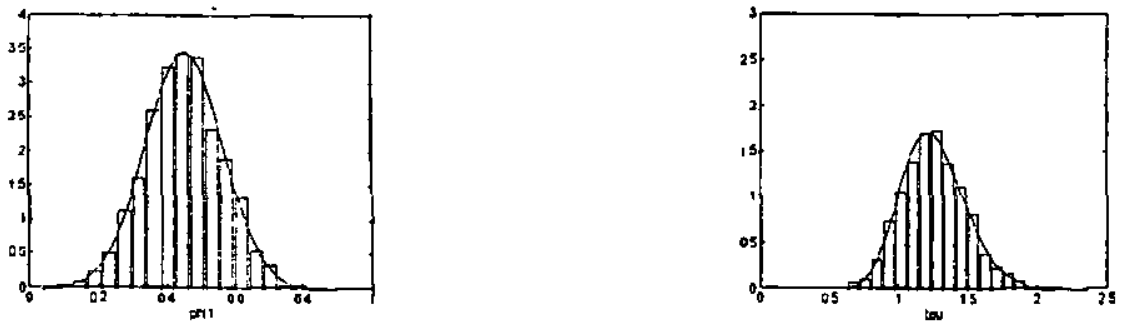

Dezembro
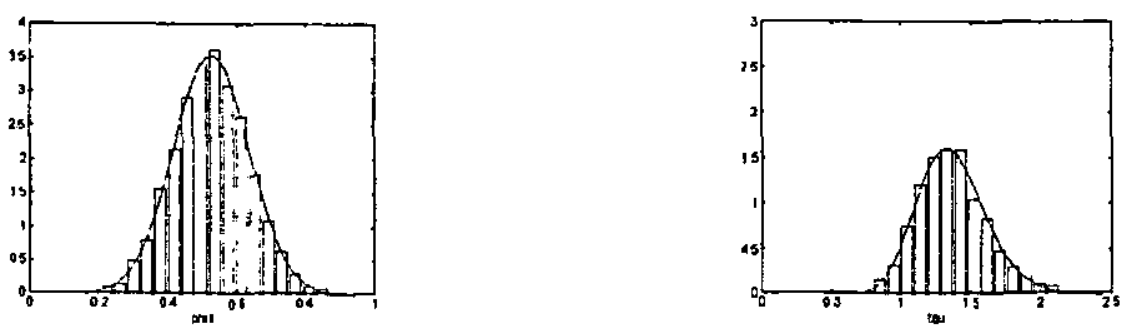

Figura 20. Densidades a Posteriori Marginais para a série de Emborcação considerando a priori Não - Informativa para os meses de Julho a Dezembro respectivamente. 
Janeiro
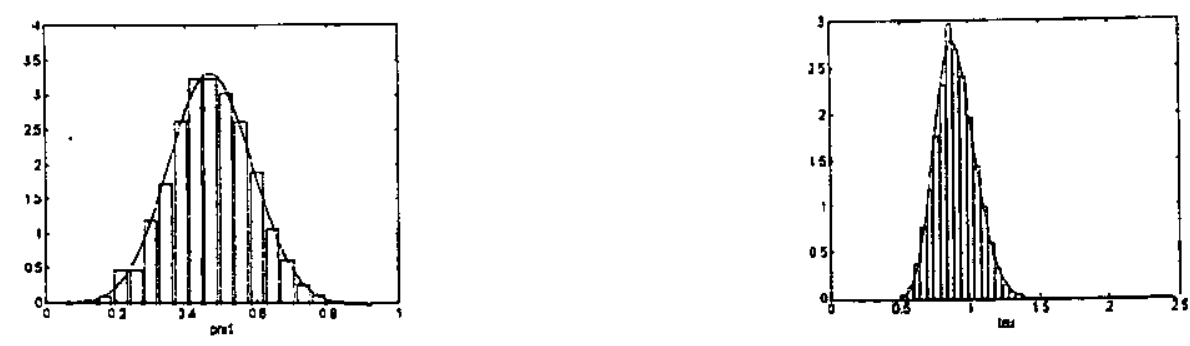

Fevereiro
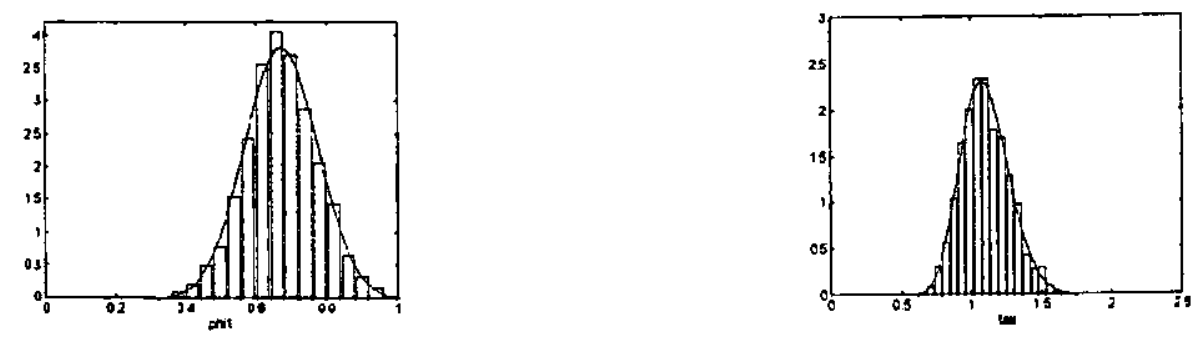

Março
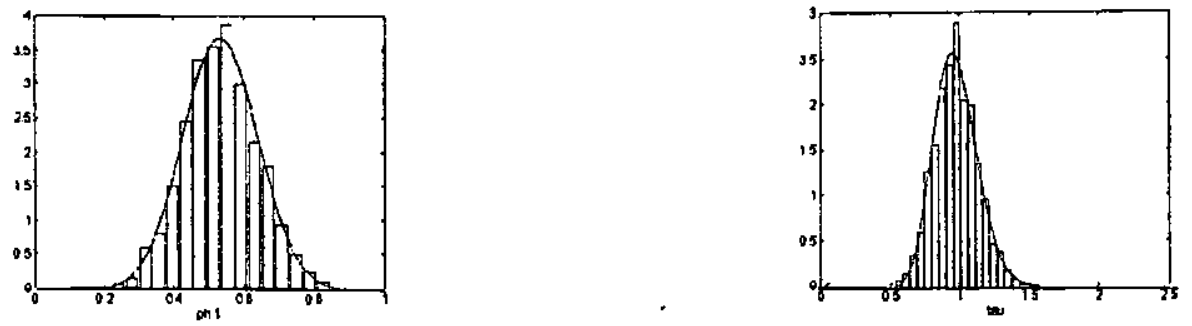

Abril
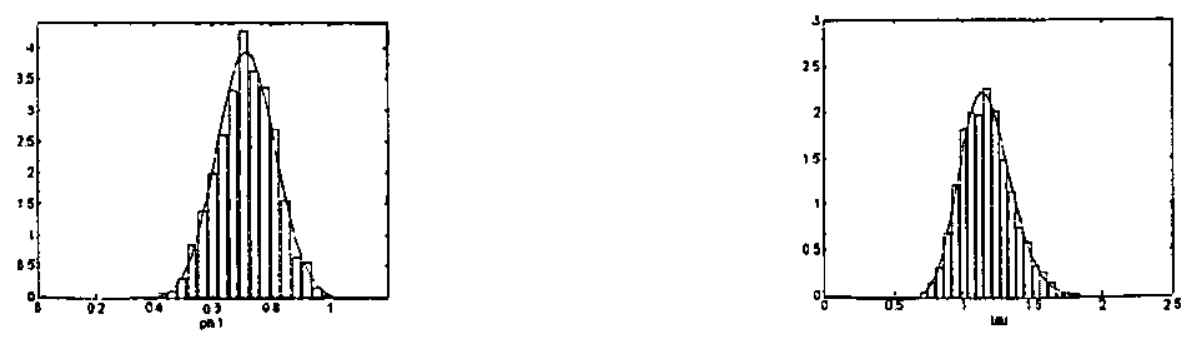

Maio
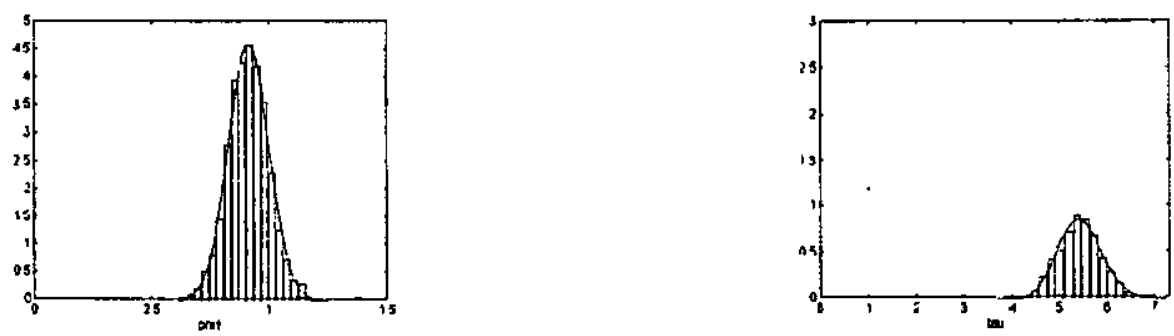

- Junho
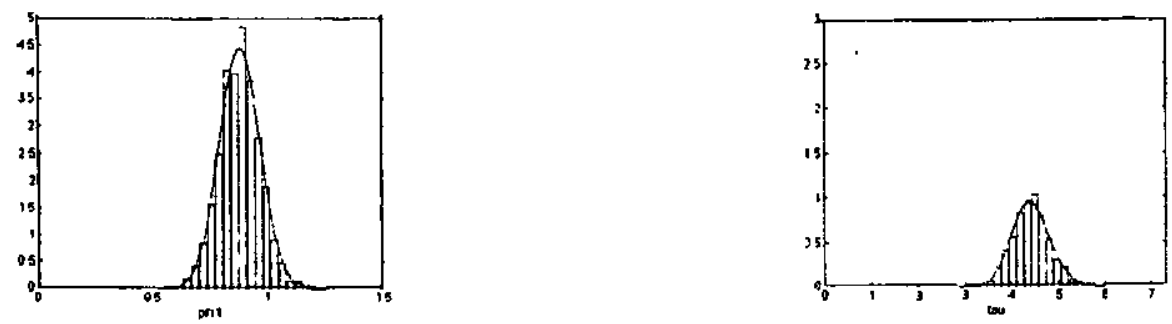

Figura 21. Densidades a Posteriori Marginais para a série de Emborcação considerando a priori Normal-Gama para os meses de Janeiro a Junho respectivamente. 
Julho
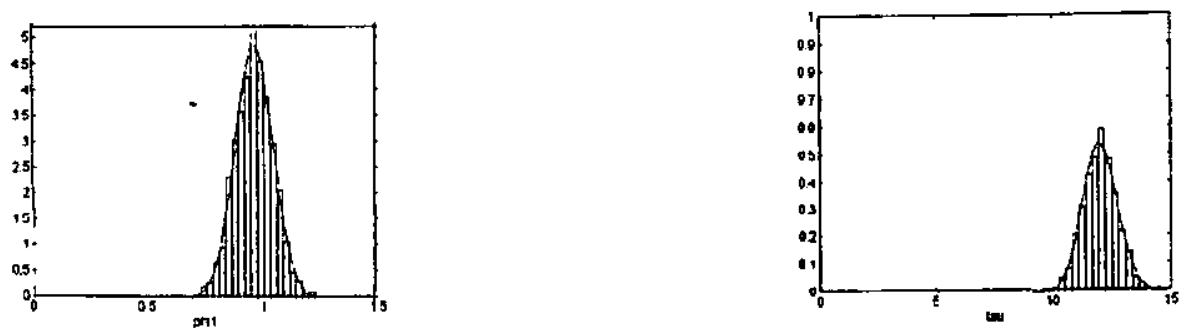

Agosto
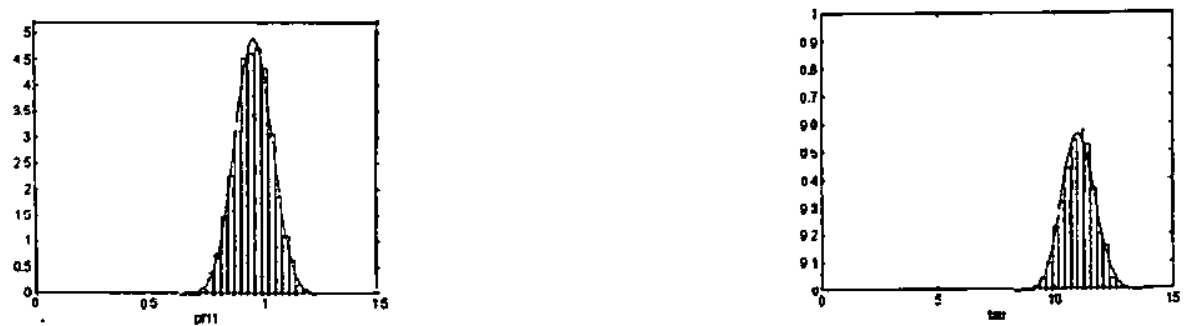

Setembro
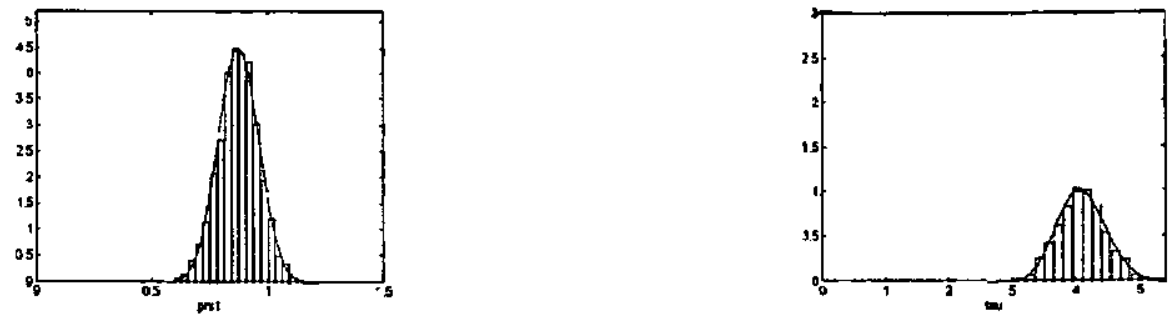

Outubro
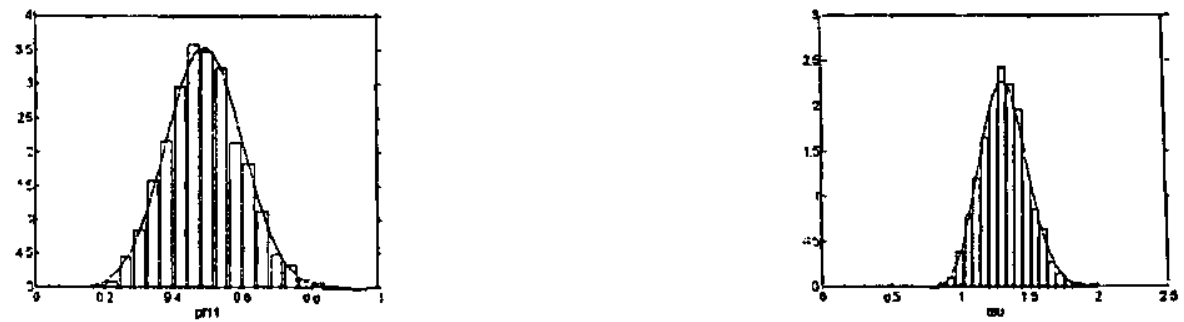

Novembro
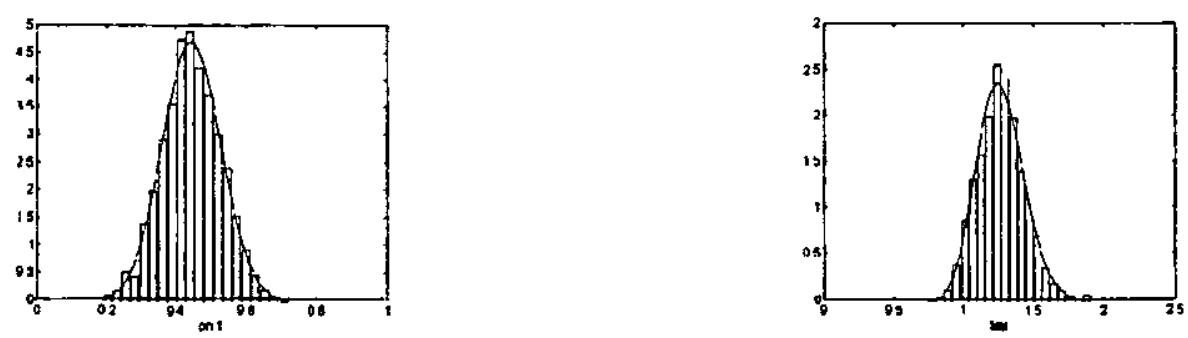

Dezembro
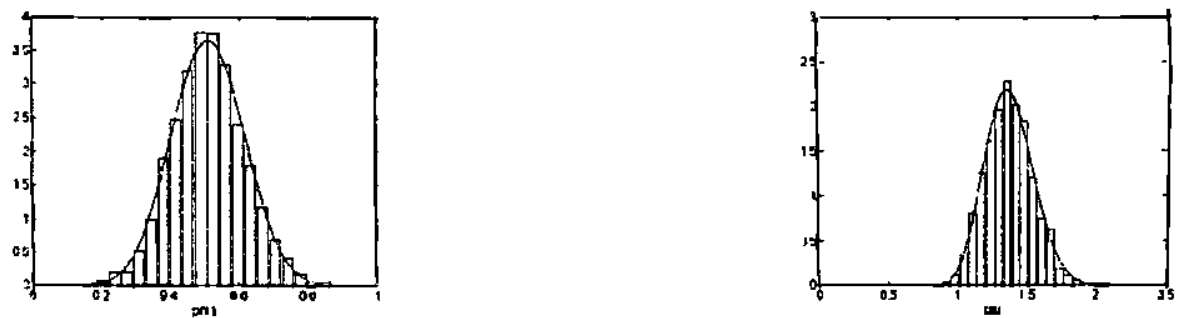

Figura 22. Densidades a Posteriori Marginais para a série de Emborcação considerando a priori Normal-Gama para os meses de Julho a Dezembro respectivamente. 
Janeiro
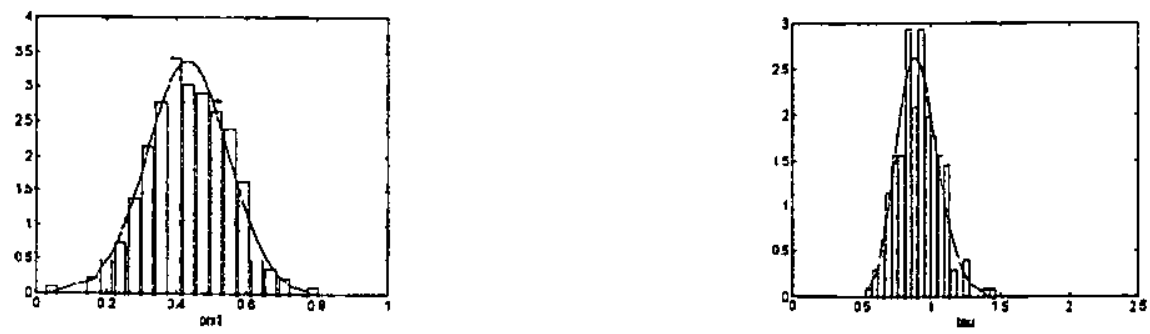

Fevereiro
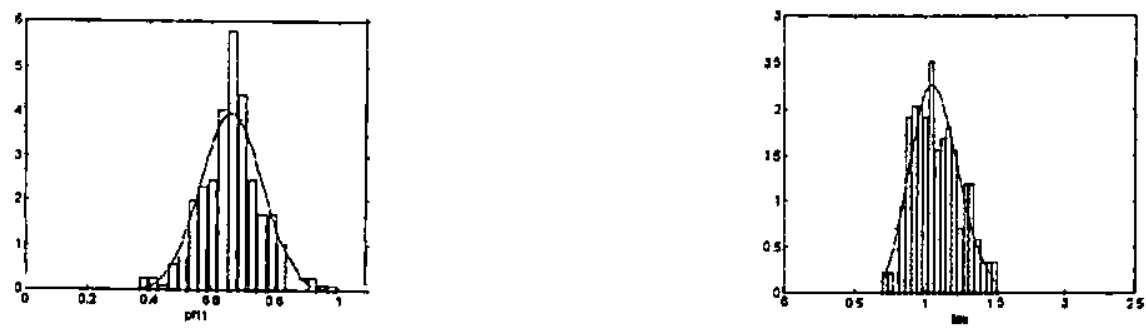

Março
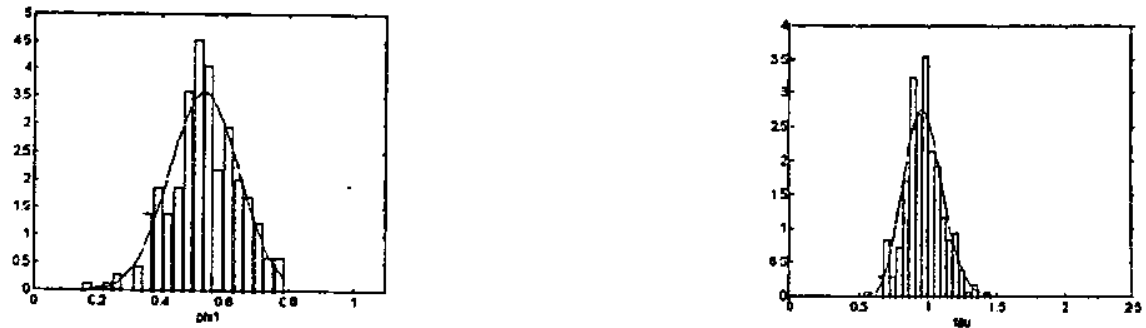

Abril
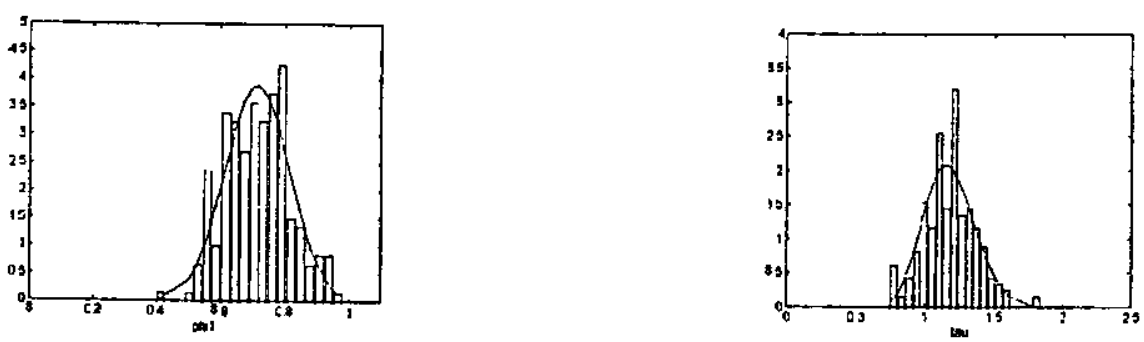

Maio
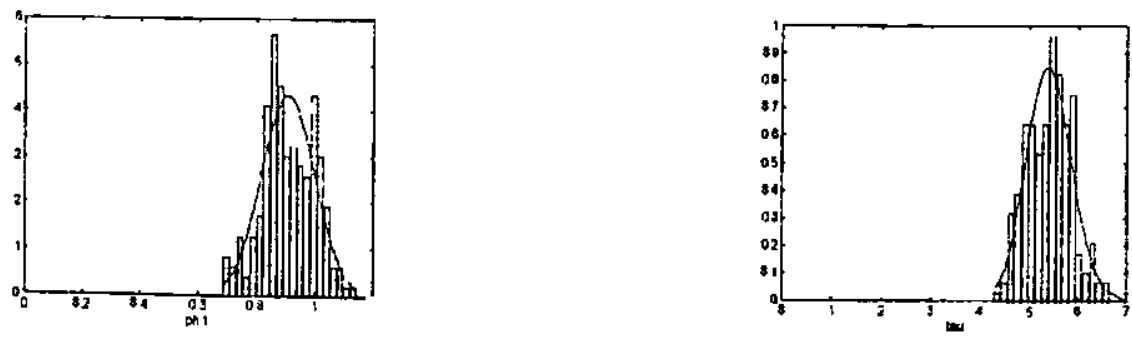

Junho
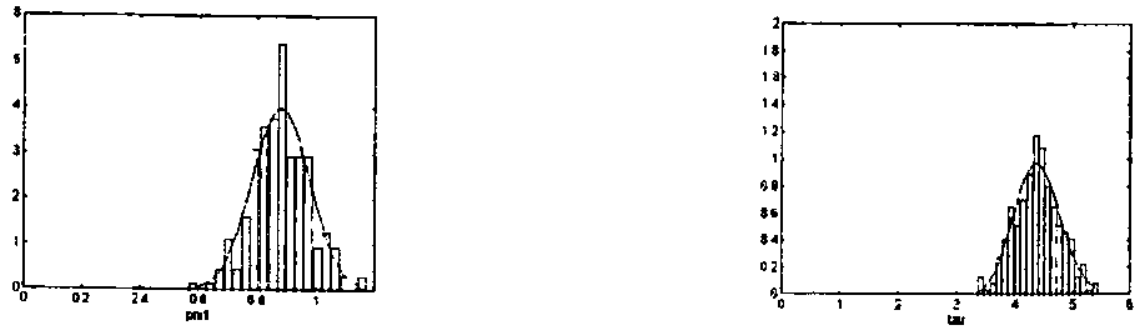

Figura 23. Densidades a Posteriori Marginais para a série de Emborcação considerando a priori t-Student e Gama para os meses de Janeiro a Junho respectivamente. 
Julho
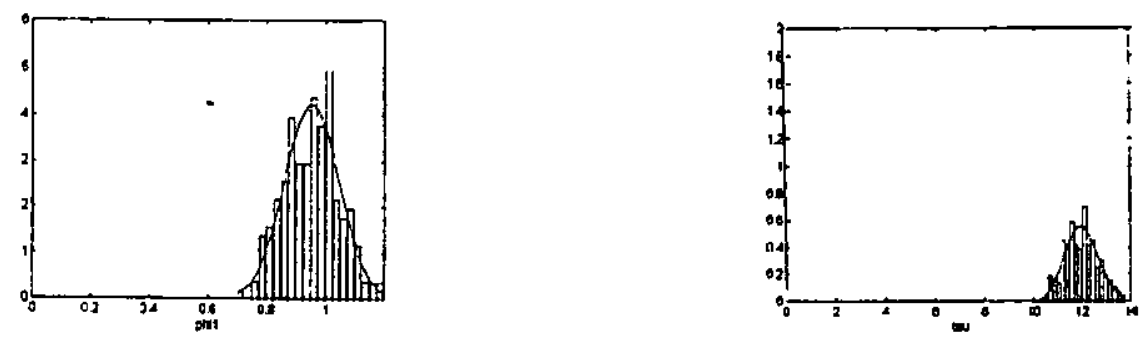

Agosto
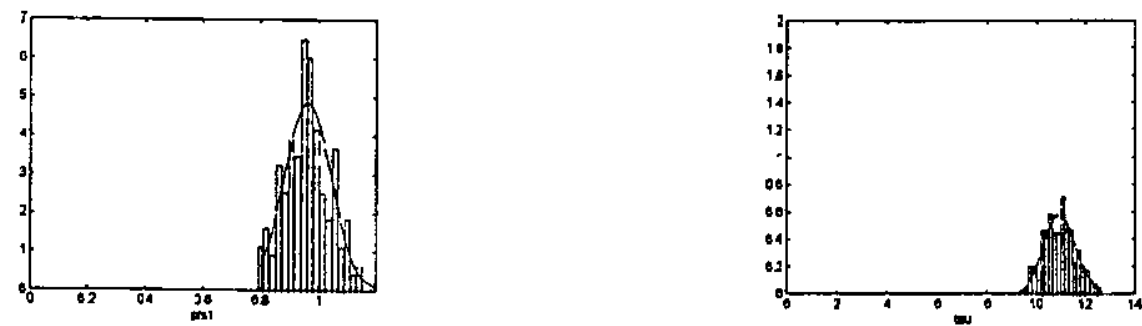

Setembro
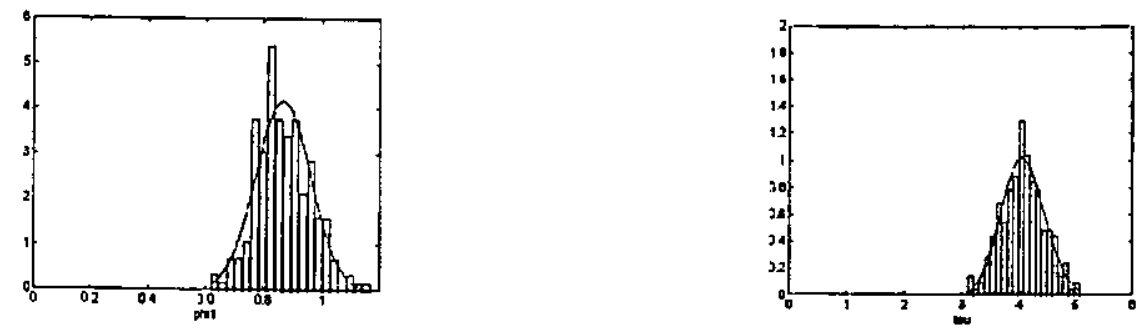

Outubro
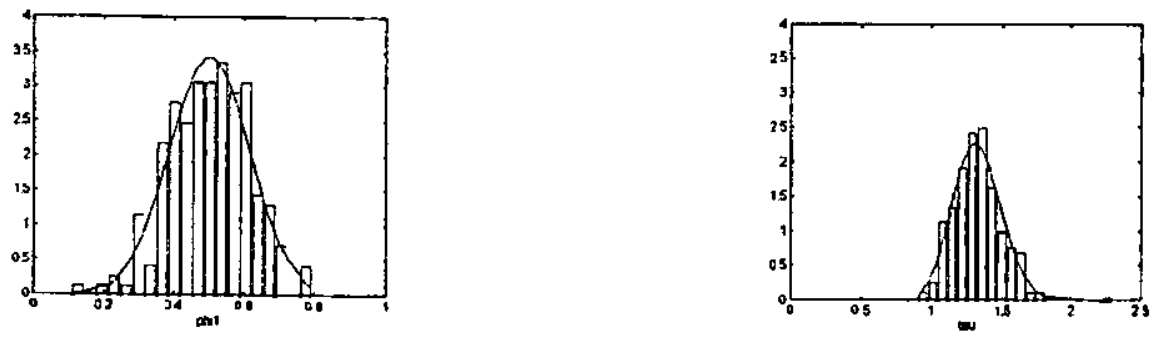

Novembro
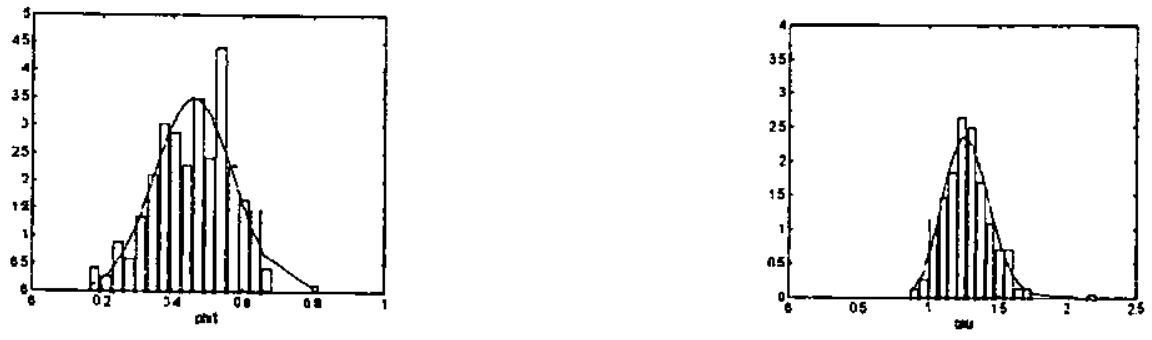

Dezembro
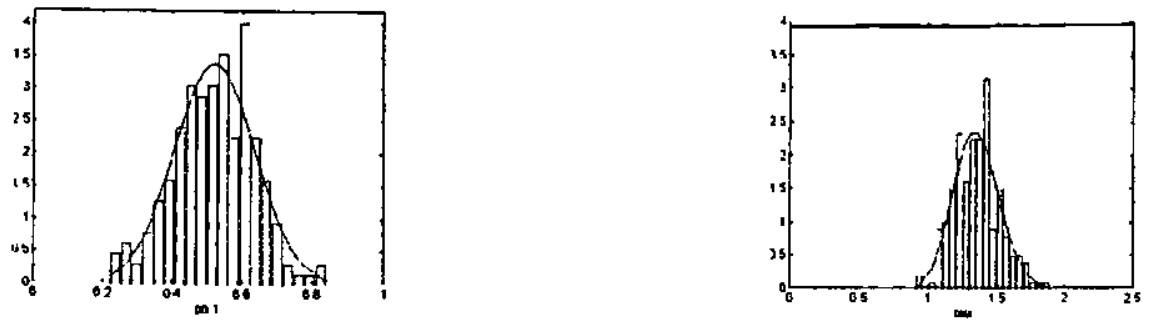

Figura 24. Densidades a Posteriori Marginais para a série de Emborcação considerando a priori t-Student e Gama para os meses de Julho a Dezembro respectivament 
Novamente, no caso do produto das priori $t$-Student e Gama, onde utilizamos o algoritmo de Metropolis-Hastings para verificar a independência dos dados construímos o gráfico da correlação dos 6000 pontos amostrais de $\phi_{1, m}$ e $\tau_{m}$, ver Figura 25.

Observamos através da Figura 25 uma alta correlação entre os dados da amostra selecionada. Portanto consideramos a seleção espaçada, ver Figura 26.

Observamos somente as correlações do mês de Junho, pois para os demais meses as correlações são semelhantes, por isso são omitidas.

A convergência dos dados da série de Emborcação são apresentados nas Figuras 27,28 e 29 respectivamente.

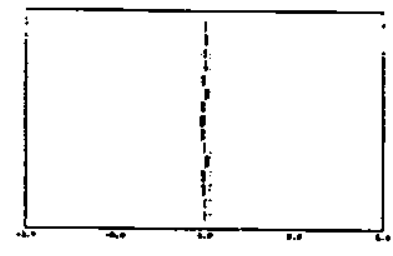

$\tau_{6}$

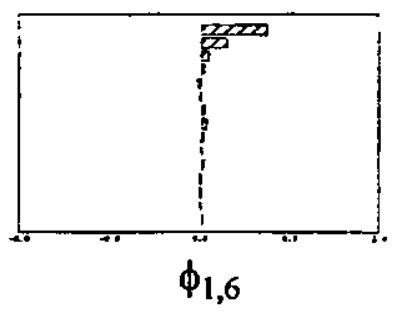

Figura 25. Correlação da amostra gerada pelo amostrador de Gibbs com Metropolis de $\tau_{\mathrm{m}}, \mathrm{e} \phi_{1, \mathrm{~m}}$ para o mês de Junho considerando os 3000 pontos finais da seqüência.

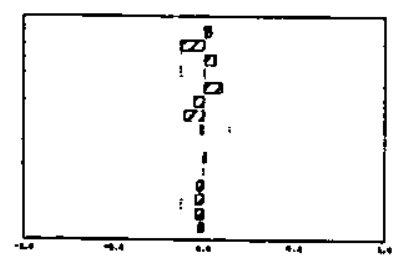

$\tau_{6}$

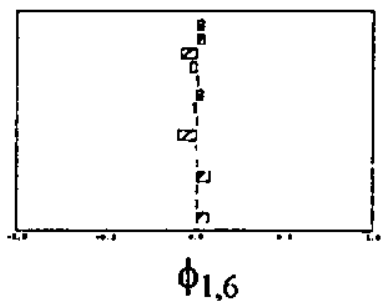

$\phi_{1,6}$

Figura 26. Correlação da amostra de $\tau_{m}$, e $\phi_{1, m}$ para o mês de Junho, desprezando os 3000 valores iniciais da última seqüência e selecionando-se os 200 pontos restantes de 15 em 15. 

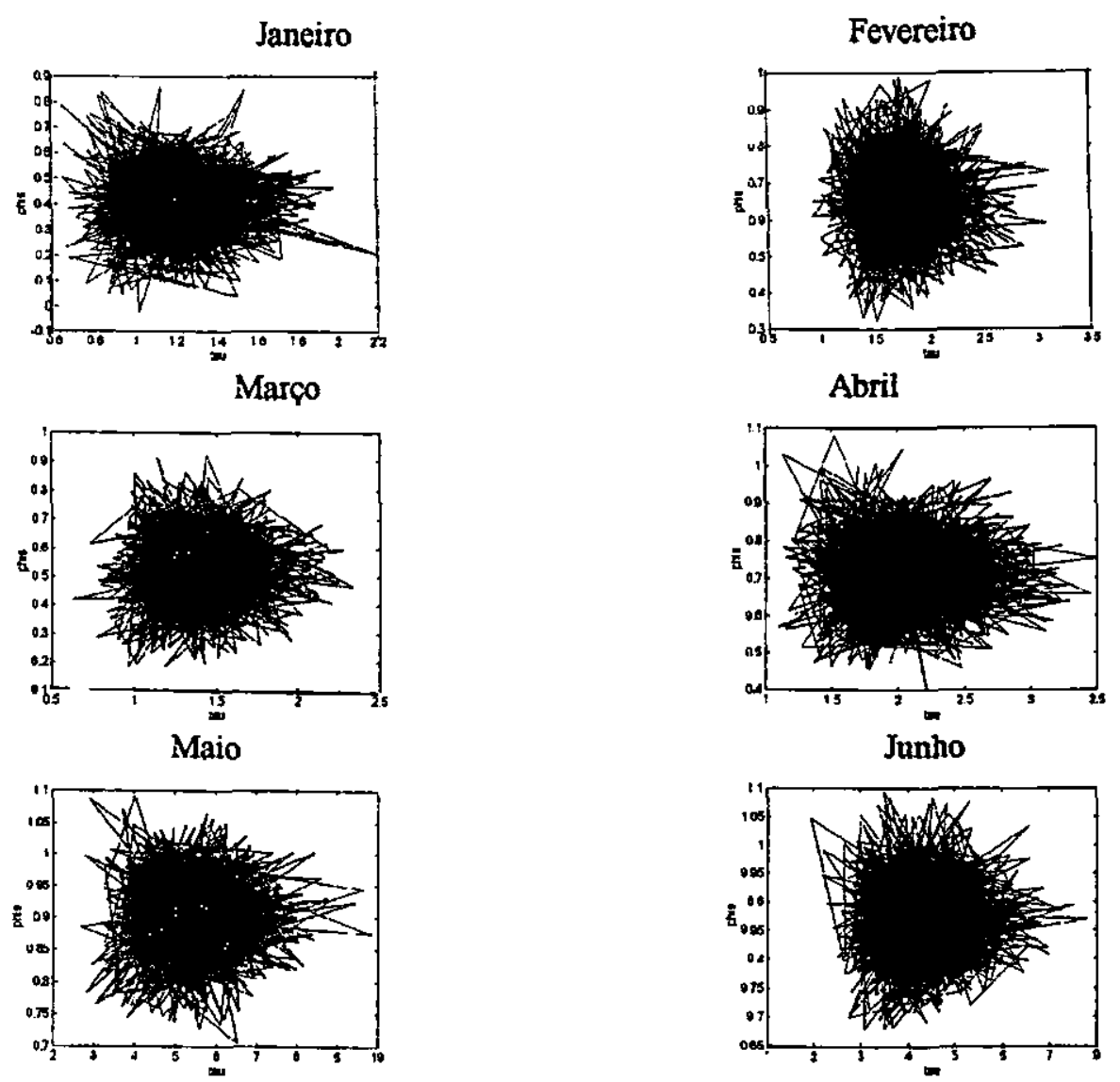

Julho
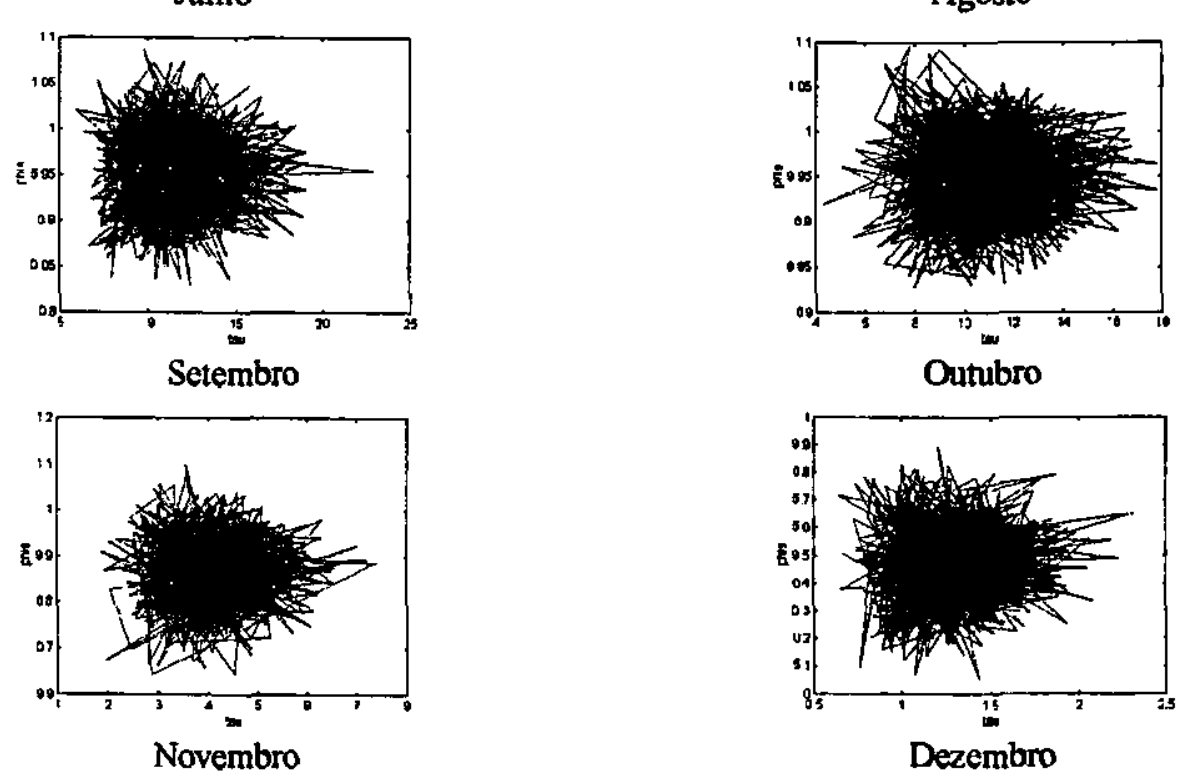

Dezembro
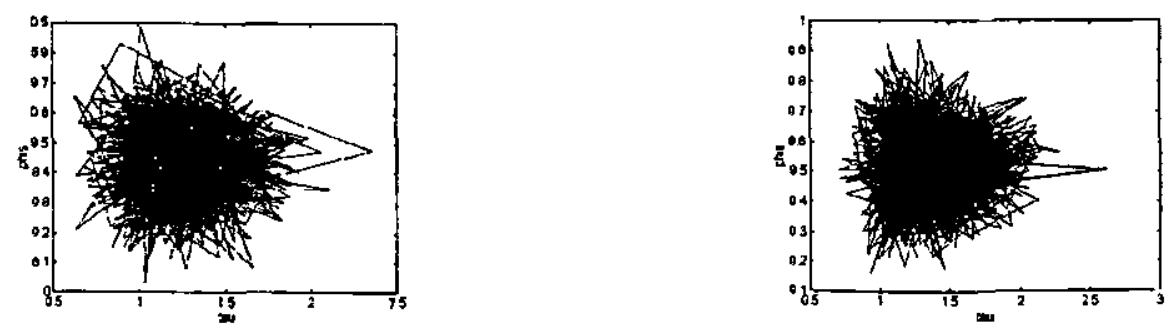

Figura 27. Convergência para a série de Emborcação considerando a priori Não - Informativa para os meses de Janeiro a Dezembro respectivamente. 


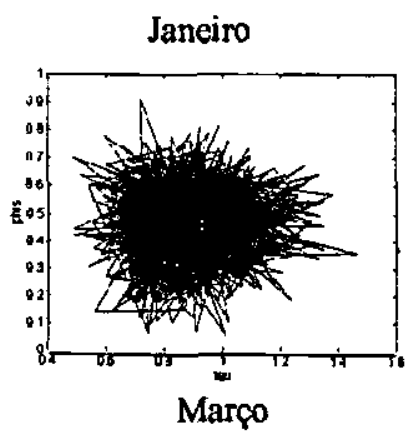

Fevereiro
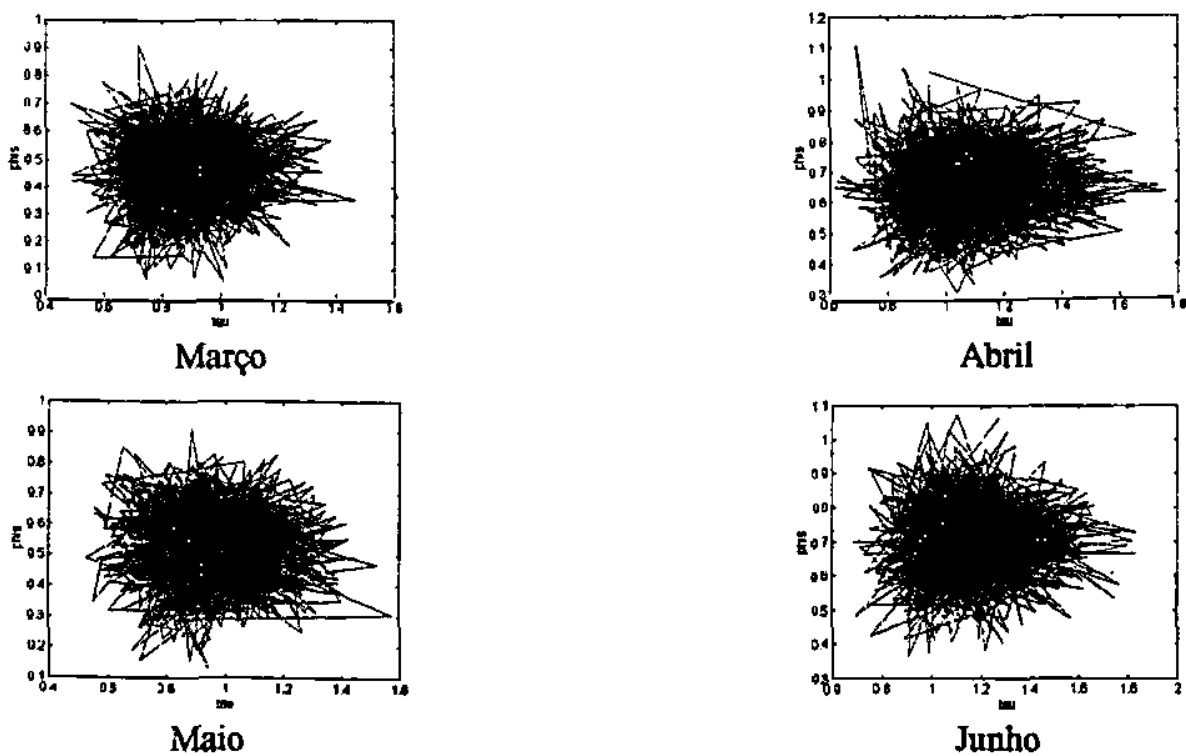

Abril
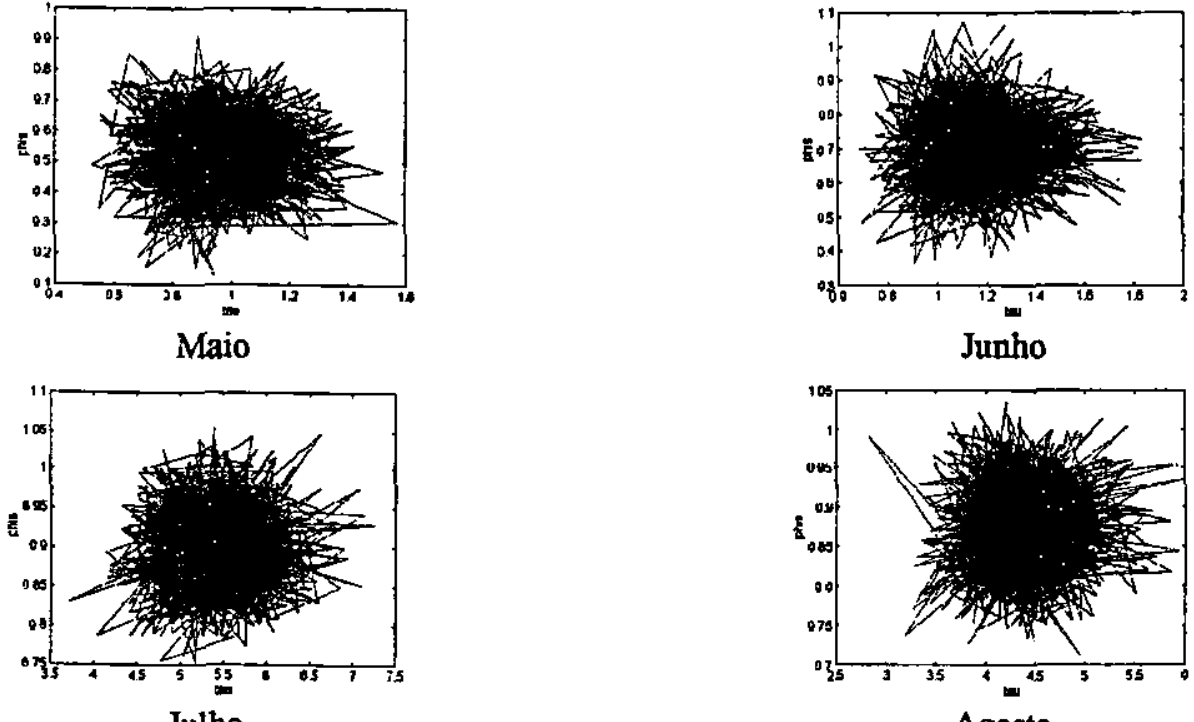

Agosto
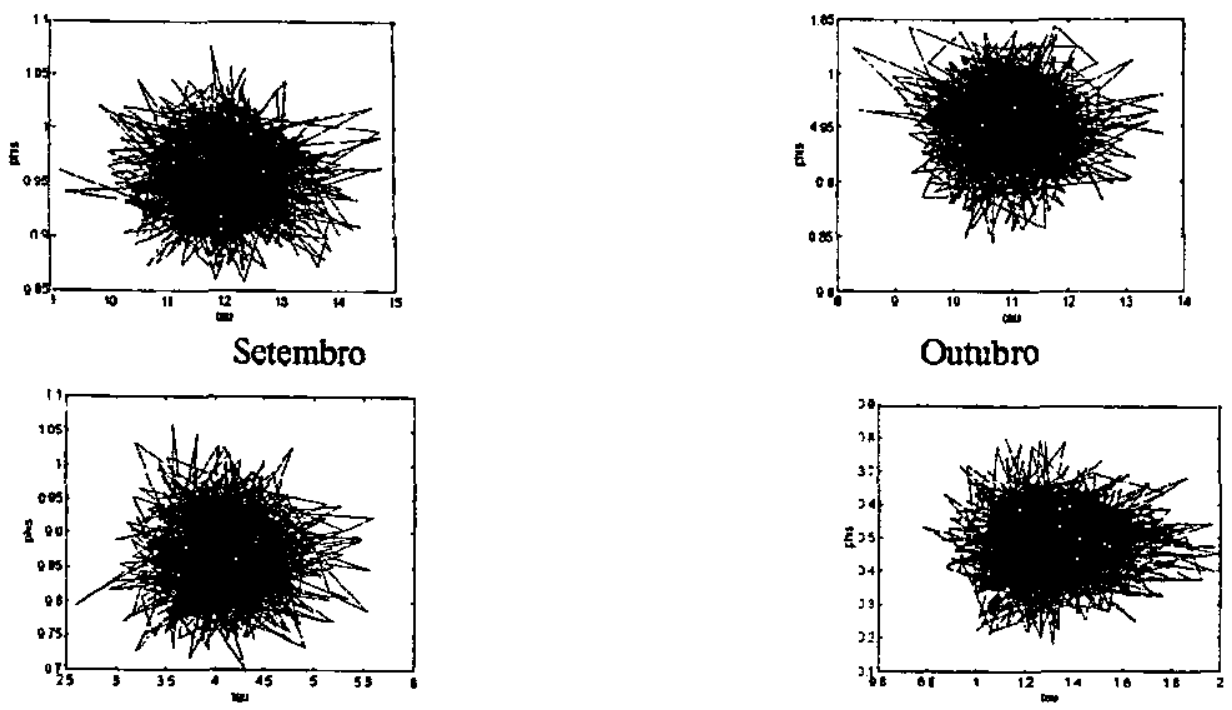

Novembro

Dezembro
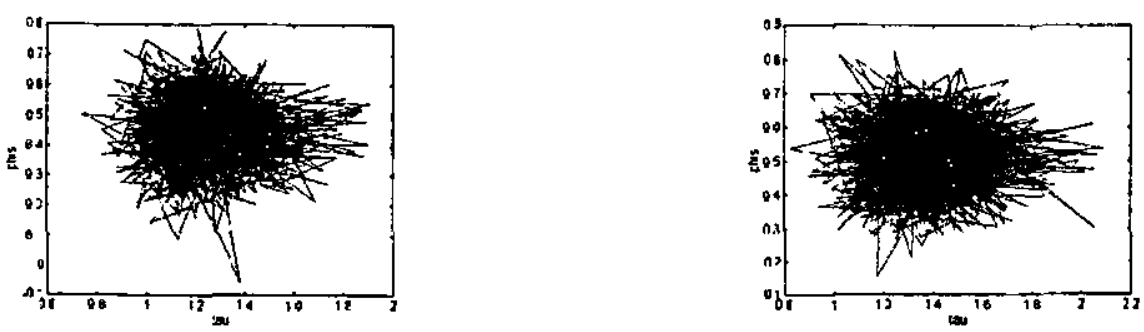

Figura 28. Convergência para a série de Emborcação considerando a priori NormalGama para os meses de Janeiro a Dezembro respectivamente. 

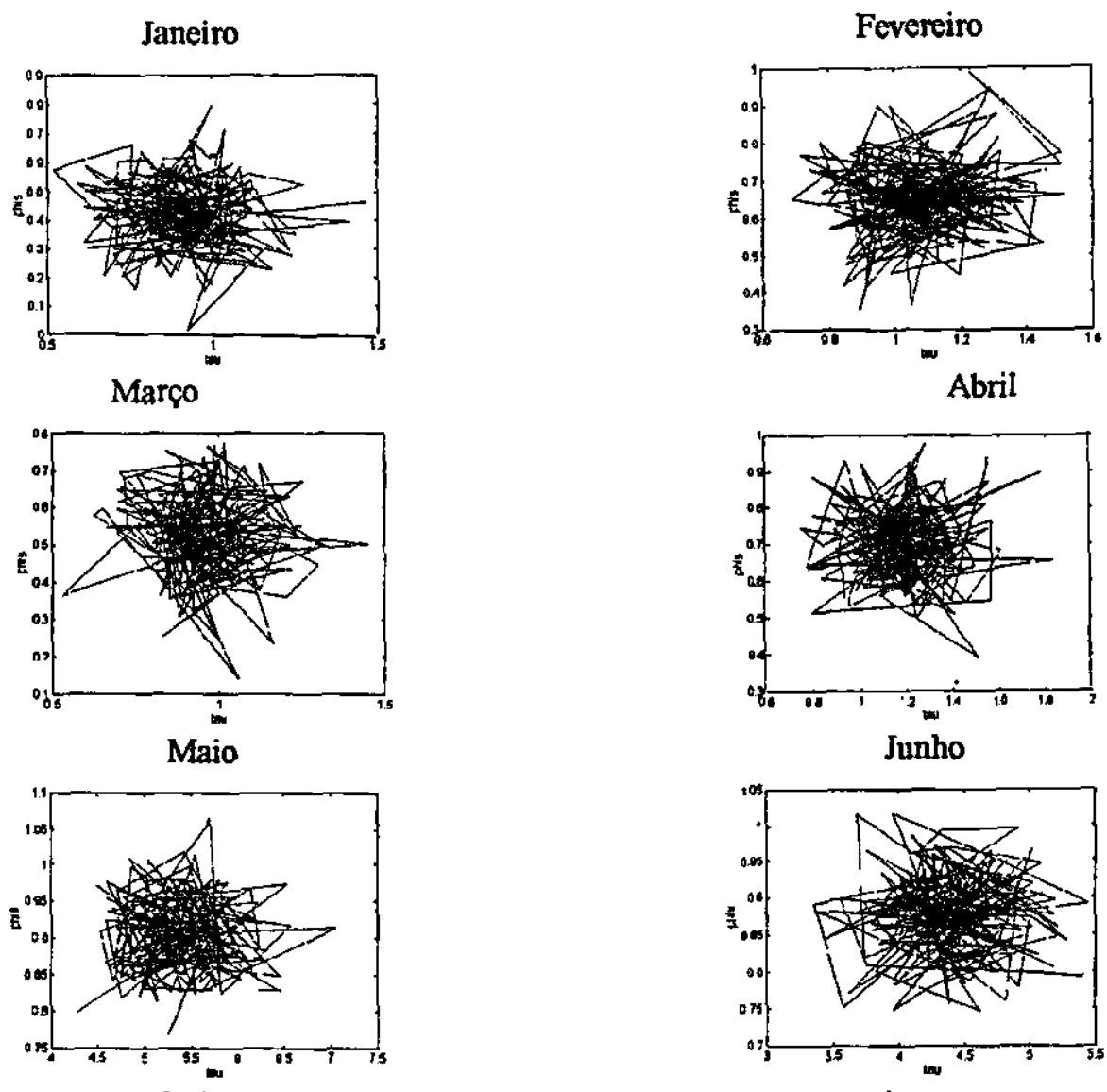

Julho

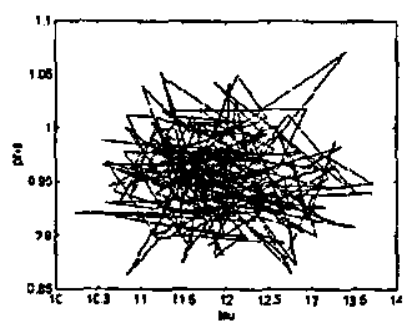

Agosto
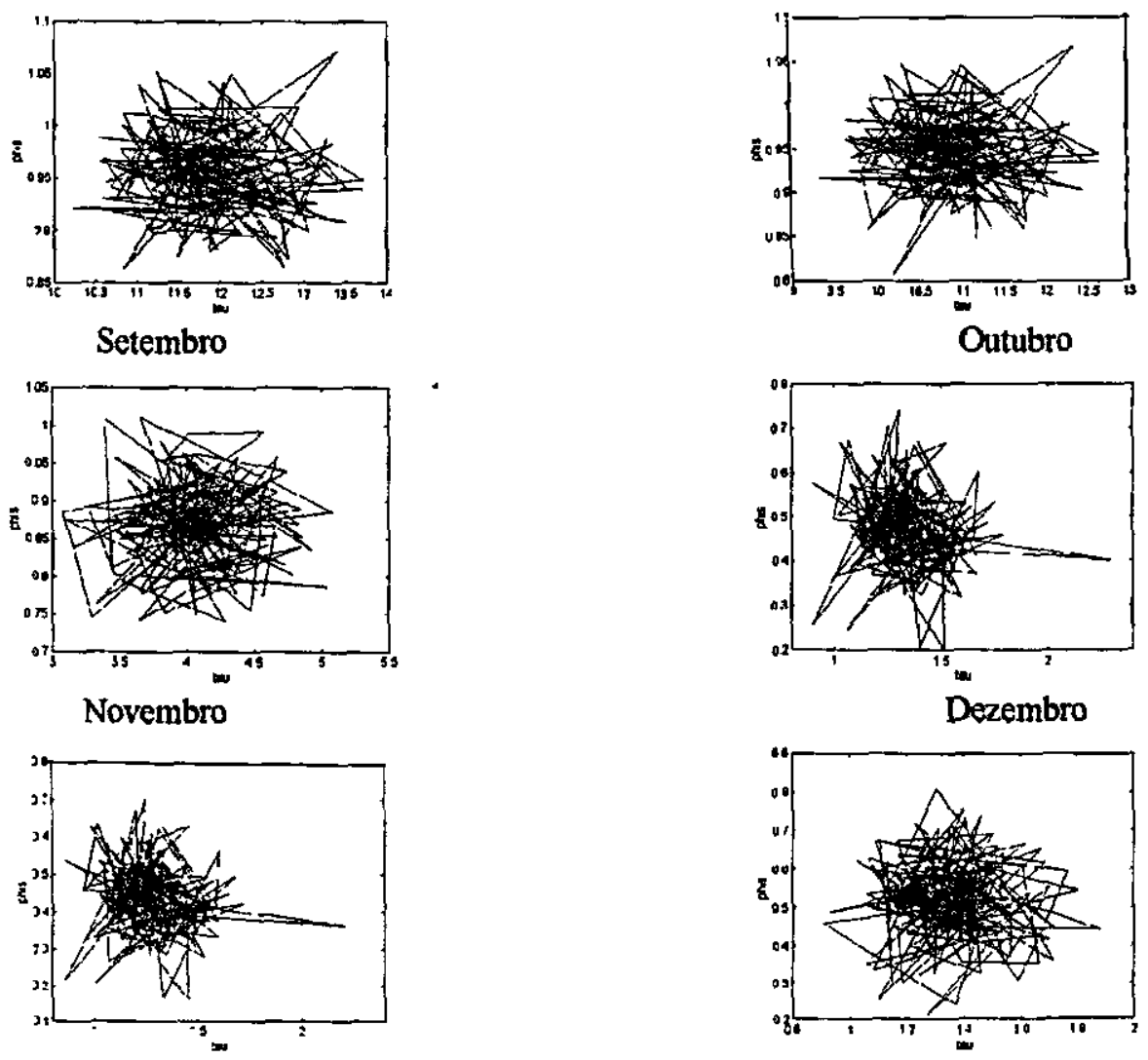

Figura 29. Convergência para a série de Emborcação considerando a priori t-Student e Gama para os meses de Janeiro a Dezembro respectivamente. 
Os resultados dos estimadores dos parâmetros para os meses de Janeiro a Dezembro, considerando as três densidades a priori analisadas neste trabalho são apresentadas na Tabelas 23 a 34 a seguir.

Tabela 23. Valores Estimados dos parâmetros para o mês de Janeiro

\begin{tabular}{l|llllll}
\hline & $\tau_{m}$ & $D P$ & $R$ & $\phi_{1, m}$ & $D P$ & $R$ \\
\hline EMV & 1,2030 & 0,2215 & & 0,4172 & 0,1206 & \\
PNI(EX) & 1,2030 & 0,2215 & & 0,4172 & 0,1166 & \\
PNI(GS) & 1,2110 & 0,2247 & 1,0014 & 0,4124 & 0,1214 & 1,0006 \\
PNG(EX) & 0,9487 & 0,1519 & & 0,5075 & 0,1101 & \\
PNG(GS) & 0,9653 & 0,1489 & 1,0007 & 0,5075 & 0,1082 & 1,0004 \\
PTSG(MH) & 0,9015 & 0,1530 & 1,0261 & 0,4290 & 0,1171 & 0,9997 \\
\hline
\end{tabular}

Tabela 24. Valores Estimados dos parâmetros para o mês de Fevereiro

\begin{tabular}{l|cccccc}
\hline & $\tau_{m}$ & $D P$ & $R$ & $\phi_{l, m}$ & $D P$ & $R$ \\
\hline EMV & 1,7657 & 0,3251 & & 0,6585 & 0,0980 & \\
PNI(EX) & 1,7657 & 0,3251 & & 0,6585 & 0,0948 & \\
PNI(GS) & 1,7661 & 0,3177 & 0,9996 & 0,6625 & 0,0998 & 1,0019 \\
PNG(EX) & 0,8825 & 0,1413 & & 0,4642 & 0,1214 & \\
PNG(GS) & 0,8929 & 0,1434 & 1,0003 & 0,4654 & 0,1193 & 0,9996 \\
PTSG(MH) & 1,0688 & 0,1779 & 0,9987 & 0,6581 & 0,0995 & 0,9970 \\
\hline
\end{tabular}

Tabela 25. Valores Estimados dos parâmetros para o mês de Março

\begin{tabular}{l|cccccc}
\hline & $\tau_{m}$ & $D P$ & $R$ & $\phi_{1, m}$ & $D P$ & $R$ \\
\hline EMV & 1,4002 & 0,2578 & & 0,5346 & 0,1100 & \\
PNI(EX) & 1,4002 & 0,2578 & & 0,5346 & 0,1064 & \\
PNI(GS) & 1,3971 & 0,2529 & 0,9999 & 0,5347 & 0,1152 & 1,0004 \\
PNG(EX) & 1,0812 & 0,1731 & & 0,6686 & 0,1028 & \\
PNG(GS) & 1,0957 & 0,1741 & 1,0010 & 0,6678 & 0,1039 & 1,0002 \\
PTSG(MH) & 0,9597 & 0,1441 & 1,0115 & 0,5311 & 0,1106 & 1,0009 \\
\hline
\end{tabular}

Tabela 26. Valores Estimados dos parâmetros para o mês de Abril

\begin{tabular}{l|cccccc}
\hline & $\tau_{m}$ & $D P$ & $R$ & $\phi_{l m}$ & $D P$ & $R$ \\
\hline EMV & 2,0606 & 0,3794 & & $\mathbf{0 , 7 1 7 4}$ & 0,0907 & \\
PNI(EX) & 2,0606 & 0,3794 & & 0,7174 & 0,0877 & \\
PNI(GS) & 2,0653 & 0,3752 & 0,9999 & 0,7167 & 0,0920 & 0,9997 \\
PNG(EX) & 0,9515 & 0,1524 & & 0,5292 & 0,1097 & \\
PNG(GS) & 0,9649 & 0,1552 & 0,9997 & 0,5295 & 0,1075 & 1,0009 \\
PTSG(MH) & 1,1752 & 0,1904 & 0,9985 & 0,7120 & 0,1016 & 1,0063 \\
\hline
\end{tabular}


Tabela 27. Valores Estimados dos parâmetros para o mês de Maio

\begin{tabular}{l|cccccc}
\hline & $\tau_{m}$ & $D P$ & $R$ & $\phi_{1, m}$ & $D P$ & $R$ \\
\hline EMV & 5,4412 & 1,0018 & & 0,9034 & 0,0558 & \\
PNI(EX) & 5,4412 & 1,0018 & - & 0,9034 & 0,0540 & \\
PNI(GS) & 5,4314 & 0,9941 & 1,0003 & 0,9042 & 0,0569 & 1,0005 \\
PNG(EX) & 5,4285 & 0,4621 & & 0,9034 & 0,0457 & \\
PNG(GS) & 5,4212 & 0,4621 & 1,0001 & 0,9026 & 0,0465 & 0,9996 \\
PTSG(MH) & 5,3862 & 0,4692 & 1,0114 & 0,9058 & 0,0909 & 1,0005 \\
\hline
\end{tabular}

Tabela 28. Valores Estimados dos parâmetros para o mês de Junho

\begin{tabular}{l|cccccc}
\hline & $\tau_{m}$ & $D P$ & $R$ & $\phi_{1, m}$ & $D P$ & $R$ \\
\hline EMV & 4,3378 & 0,7987 & & 0,8772 & 0,0625 & \\
PNI(EX) & 4,3378 & 0,7987 & & 0,8772 & 0,0605 & \\
PNI(GS) & 4,3442 & 0,8118 & 1,0008 & 0,8776 & 0,0632 & 1,0006 \\
PNG(EX) & 4,4029 & 0,4053 & & 0,9109 & 0,0864 & \\
PNG(GS) & 4,3993 & 0,4058 & 1,0003 & 0,9111 & 0,0862 & 1,0002 \\
PTSG(MH) & 4,3833 & 0,4059 & 1,0074 & 0,8805 & 0,0550 & 0,9990 \\
\hline
\end{tabular}

Tabela 29. Valores Estimados dos parâmetros para o mês de Julho

\begin{tabular}{l|cccccc}
\hline & $\tau_{m}$ & $D P$ & $R$ & $\phi_{l, m}$ & $D P$ & $R$ \\
\hline EMV & 11,815 & 2,1754 & & 0,9567 & 0,0379 & \\
PNI(EX) & 11,815 & 2,1754 & & 0,9567 & 0,0366 & \\
PNI(GS) & 11,784 & 2,1721 & 0,9997 & 0,9559 & 0,0390 & 1,0014 \\
PNG(EX) & 12,001 & 0,7304 & & 0,8760 & 0,0885 & \\
PNG(GS) & 11,989 & 0,7331 & 0,9998 & 0,8760 & 0,0888 & 0,9997 \\
PTSG(MH) & 11,924 & 0,7103 & 1,0044 & 0,9500 & 0,0937 & 0,9998 \\
\hline
\end{tabular}

Tabela 30. Valores Estimados dos parâmetros para o mês de Agosto

\begin{tabular}{l|cccccc}
\hline & $\tau_{m}$ & $D P$ & $R$ & $\phi_{1, m}$ & $D P$ & $R$ \\
\hline EMV & 10,846 & 1,9969 & & 0,9528 & 0,0395 & \\
PNI(EX) & 10,846 & 1,9969 & & 0,9528 & 0,0382 & \\
PNI(GS) & 10,900 & 1,9696 & 1,0007 & 0,9524 & 0,0400 & 0,9997 \\
PNG(EX) & 11,003 & 0,6959 & & 0,9603 & 0,0810 & \\
PNG(GS) & 10,995 & 0,6985 & 1,0002 & 0,9589 & 0,0819 & 0,9997 \\
PTSG(MH) & 10,925 & 0,6771 & 1,0022 & 0,9509 & 0,0425 & 0,9961 \\
\hline
\end{tabular}


Tabela 31. Valores Estimados dos parâmetros para o mês de Setembro

\begin{tabular}{l|cccccc}
\hline & $\tau_{m}$ & $D P$ & $R$ & $\phi_{l, m}$ & $D P$ & $R$ \\
\hline EMV & 4,1148 & 0,7576 & & 0,8700 & 0,0642 & \\
PNI(EX) & 4,1158 & 0,7576 & - & 0,8700 & 0,0621 & \\
PNI(GS) & 4,1125 & 0,7628 & 0,9995 & 0,8717 & 0,0640 & 1,0004 \\
PNG(EX) & 4,0835 & 0,3878 & & 0,8700 & 0,0527 & \\
PNG(GS) & 4,0826 & 0,3890 & 1,0035 & 0,8625 & 0,0511 & 0,9996 \\
PTSG(MH) & 4,0648 & 0,3879 & 0,9967 & 0,8729 & 0,0564 & 0,9984 \\
\hline
\end{tabular}

Tabela 32. Valores Estimados dos parâmetros para o mês de Outubro

\begin{tabular}{l|cccccc}
\hline & $\tau_{m}$ & $D P$ & $R$ & $\phi_{l, m}$ & $D P$ & $R$ \\
\hline EMV & 1,3078 & 0,2408 & & 0,4851 & 0,1138 & \\
PNI(EX) & 1,3078 & 0,2408 & & 0,4851 & 0,1101 & \\
PNI(GS) & 1,3025 & 0,2393 & 1,0006 & 0,4833 & 0,1170 & 1,0003 \\
PNG(EX) & 1,3159 & 0,1758 & & 0,4851 & 0,0932 & \\
PNG(GS) & 1,3154 & 0,1762 & 1,0018 & 0,4872 & 0,0939 & 0,9997 \\
PTSG(MH) & 1,3209 & 0,1802 & 0,9968 & 0,4822 & 0,0919 & 0,9990 \\
\hline
\end{tabular}

Tabela 33. Valores Estimados dos parâmetros para o mês de Novembro

\begin{tabular}{l|cccccc}
\hline & $\tau_{m}$ & $D P$ & $R$ & $\phi_{l, m}$ & $D P$ & $R$ \\
\hline EMV & 1,2513 & 0,2304 & & 0,4482 & 0,1164 & \\
PNI(EX) & 1,2513 & 0,2304 & & 0,4482 & 0,1126 & \\
PNI(GS) & 1,2493 & 0,2319 & 0,9995 & 0,4464 & 0,1147 & 1,0010 \\
PNG(EX) & 1,2622 & 0,1702 & & 0,4482 & 0,0952 & \\
PNG(GS) & 1,2620 & 0,1705 & 1,0003 & 0,4476 & 0,0940 & 1,0013 \\
PTSG(MH) & 1,2664 & 0,1742 & 0,9999 & 0,4444 & 0,0927 & 1,0006 \\
\hline
\end{tabular}

Tabela 34. Valores Estimados dos parâmetros para o mês de Dezembro

\begin{tabular}{l|cccccc}
\hline & $\tau_{m}$ & $D P$ & $R$ & $\phi_{l, m}$ & $D P$ & $R$ \\
\hline EMV & 1,3733 & 0,2528 & & 0,5214 & 0,1111 & \\
PNI(EX) & 1,3733 & 0,2528 & & 0,5214 & 0,1075 & \\
PNI(GS) & 1,3612 & 0,2495 & 0,9998 & 0,5225 & 0,1120 & 1,0003 \\
PNG(EX) & 1,3741 & 0,1820 & & 0,5214 & 0,0912 & \\
PNG(GS) & 1,3740 & 0,1821 & 1,0004 & 0,5250 & 0,0905 & 1,0000 \\
PTSG(MH) & 1,3487 & 0,1692 & 1,0038 & 0,5243 & 0,1050 & 1,0088 \\
\hline
\end{tabular}


$\mathrm{Na}$ Tabela 35 apresentamos o intervalo de credibilidade dos dados simulados para o caso do produto das priori t-Student e Gama para a série de Emborcação.

Tabela 35. Intervalo de Credibilidade para os parâmetros $\tau_{\mathrm{m}}$, e $\phi_{1, \mathrm{~m}}$ (Priori $t$-Student e Gama- Janeiro a Dezembro)

\begin{tabular}{l|cc}
\hline mes $m$ & \multicolumn{1}{|c}{$\tau_{\mathrm{m}}$} & $\phi_{1, \mathrm{~m}}$ \\
\hline Janeiro $(\mathrm{m}=1)$ & $(0,6156 ; 1,2318)$ & $(0,2087 ; 0,6479)$ \\
Fevereiro $(\mathrm{m}=2)$ & $(0,7710 ; 1,4295)$ & $(0,4549 ; 0,8805)$ \\
Março $(\mathrm{m}=3)$ & $(0,9597 ; 0,1441)$ & $(0,5311 ; 0,1106)$ \\
Abril $(\mathrm{m}=4)$ & $(0,7875 ; 1,5510)$ & $(0,5341 ; 0,9264)$ \\
Maio $(\mathrm{m}=5)$ & $(1,0624 ; 2,0639)$ & $(0,7162 ; 1,0661)$ \\
Junho $(\mathrm{m}=6)$ & $(1,0087 ; 1,9637)$ & $(0,6805 ; 1,0626)$ \\
Julho $(\mathrm{m}=7)$ & $(1,2738 ; 2,2728)$ & $(0,7821 ; 1,1309)$ \\
Agosto $(\mathrm{m}=8)$ & $(1,2283 ; 2,2857)$ & $(0,8035 ; 1,1202)$ \\
Setembro $(m=9)$ & $(1,0849 ; 1,8964)$ & $(0,6779 ; 1,0559)$ \\
Outubro $(m=10)$ & $(0,6484 ; 1,3089)$ & $(0,2685 ; 0,7053)$ \\
Novembro $(m=11)$ & $(0,6460 ; 1,1903)$ & $(0,2225 ; 0,6418)$ \\
Dezembro $(m=12)$ & $(0,6486 ; 1,2693)$ & $(0,2602 ; 0,7153)$ \\
\hline
\end{tabular}

Através dos intervalos de credibilidade dados na Tabela 35, observamos que os intervalos são siginificativamente diferentes de zero, conferindo desta forma com o $\mathrm{BIC}_{m}$, que indica modelos de ordem um, minimiza o critério para todos os meses.

Nas Figuras 19 a 22 observamos que a forma simétrica da posteriori coincide com a forma esperada teoricamente.

Observamos nas Figuras 23 e 24 que apesar de não conhecermos a distribuição a posteriori, os histogramas também se apresentam de uma forma simétrica.

Com os resultados obtidos nas Tabelas 23 a 34 , observamos que as estimativas pontuais são próximas para os parâmetros $\phi_{1, m}$ e $\tau_{m}$, considerando o método Clássico e Bayesiano com o uso da densidade a priori Não-Informativa. Quando usamos as densidades a priori informativas, as estimativas a posteriori apresentam mais variaçõs para os parâmetros $\tau_{m}$ do que para os parâmetros $\phi_{1, m}$ cuja 
estimativas são próximas em todos os casos.

Observamos através de $\mathrm{R}$ (fator de redução de escala) e pelos gráficos das Figuras 27,28 e 29 que a convergência é alcançada para as três densidades a priori.

\subsection{Aplicações para o Exemplo 3}

Neste exemplo, consideramos uma série gerada por um modelo PAR(1), dado por:

$$
Z_{t(r, m)}=\phi_{1, m} Z_{t(r, m)-1}+a_{t(r, m)}
$$

Os gráficos das funções de autocorrelação periódica (PeFAC) e autocorrelação parcial periódica (PeFACP) para os meses de janeiro a dezembro são mostrados a na Figura 30.

Notamos pelos gráficos da PeFAC que suas funções mudam ao longo dos meses de forma periódica, o que justifica a ajustar um modelo PAR e as funções PePACF nos mostram que podemos escolher um modelo PAR(1) para esta série gerada.

Na Tabela 36 apresentamos os resultados do critério BIC para cada mês $m$, e notamos que o melhor modelo para a série gerada é o PAR(1).

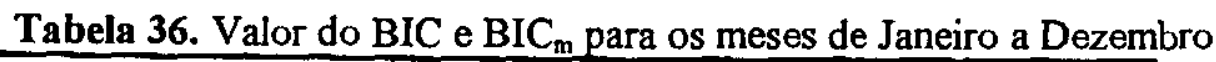

\begin{tabular}{|c|c|c|c|}
\hline & \multicolumn{3}{|c|}{$\mathrm{BIC}_{\mathrm{m}}$} \\
\hline Meses & $p_{m}=I$ & $p_{m}=2$ & $p_{m}=3$ \\
\hline Janeiro $(m=1)$ & $-0,5831$ & $-0,5149$ & $-0,4466$ \\
\hline Fevcreiro $(m=2)$ & $-0,4723$ & $-0,4041$ & $-0,3350$ \\
\hline Março $(m=3)$ & 0,09348 & 0,1616 & 0,2299 \\
\hline Abril $(m=4)$ & $-0,2113$ & $-0,1431$ & $-0,0748$ \\
\hline Maio $(m=5)$ & $-0,0227$ & 0,0454 & 0,1137 \\
\hline Junho $(m=6)$ & 0,3026 & 0,3708 & 0,4391 \\
\hline Julho $(m=7)$ & 0,0442 & 0,1124 & 0,1807 \\
\hline Agosto $(m=8)$ & 0,0425 & 0,1107 & 0,1790 \\
\hline Setembro $(m=9)$ & 0,0598 & 0,1280 & 0,1963 \\
\hline Outubro $(m=10)$ & $-0,1048$ & $-0,0366$ & 0,0316 \\
\hline Novembro $(m=11)$ & 0,2337 & 0,3019 & 0,3702 \\
\hline Dezembro $(m=12)$ & $-0,2474$ & $-0,1783$ & $-0,1092$ \\
\hline $\mathrm{BIC}=\sum_{\mathrm{m}=1}^{12} \mathrm{BIC}_{\mathrm{m}}$ & $-0,8653$ & $-0,0462$ & 0,7749 \\
\hline
\end{tabular}



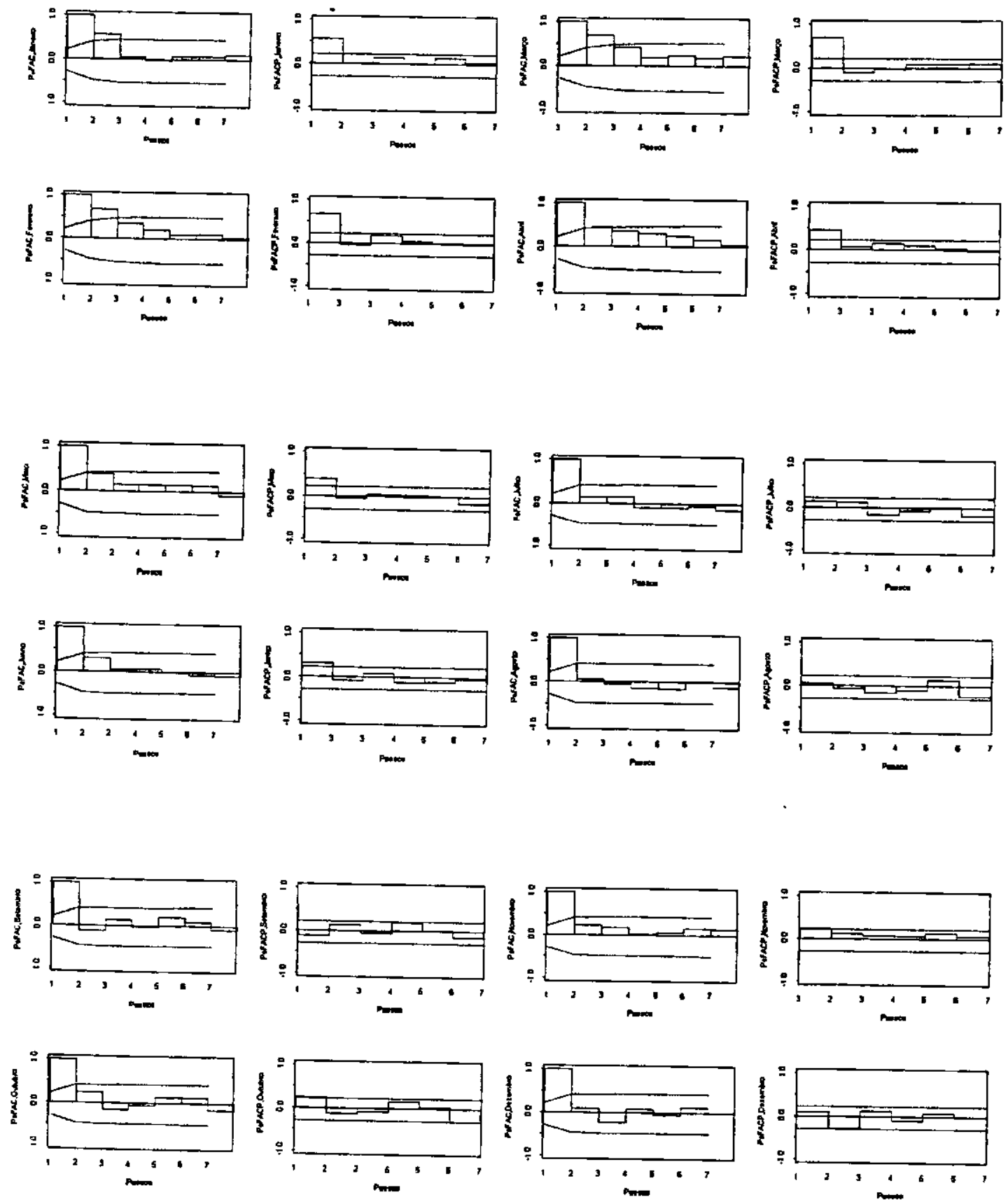

Figura 30. PeFAC e PeFACP para os meses de Janeiro a Dezembro. 
A Tabela 37 apresentam os resultados obtidos pelo critério de adequabilidade do modelo baseado na preditiva ordenada.

Tabela 37. Seleção de Modelos para a série gerada

\begin{tabular}{l|l|l|l}
\hline \multirow{2}{*}{ meses } & $p_{m}=1$ & $p_{m}=2$ & $p_{m}=3$ \\
\cline { 2 - 4 } & $\mathrm{c}(\mathrm{l})$ & $\mathrm{c}(\mathrm{l})$ & $\mathrm{c}(\mathrm{l})$ \\
\hline Janeiro $(\mathrm{m}=1)$ & 0,000036 & 0,000049 & 0,000049 \\
Fevereiro $(\mathrm{m}=2)$ & 0,0699 & 0,05789 & 0,05518 \\
Março $(\mathrm{m}=3)$ & 0,01812 & 0,01797 & 0,01978 \\
Abril $(\mathrm{m}=4)$ & 0,001813 & 0,001660 & 0,001556 \\
Maio $(\mathrm{m}=5)$ & 0,0000046 & 0,0000040 & 0,0000037 \\
Junho $(\mathrm{m}=6)$ & 0,000067 & 0,000054 & 0,000061 \\
Julho $(\mathrm{m}=7)$ & 0,0000000003 & 0,00000000036 & 0,0000000002 \\
Agosto $(\mathrm{m}=8)$ & 0,000217 & 0,00018 & 0,00026 \\
Setembro $(\mathrm{m}=9)$ & 0,00208 & 0,0032 & 0,0034 \\
Outubro $(\mathrm{m}=10)$ & 0,000010 & 0,000008 & 0,000008 \\
Novembro $(\mathrm{m}=11)$ & 0,0000017 & 0,0000013 & 0,0000012 \\
Dezembro $(\mathrm{m}=12)$ & 0,000002 & 0,000004 & 0,0000057 \\
\hline
\end{tabular}

Através das Tabelas 36 e 37 notamos que os resultados não coincidem para todos os meses, sendo necessário uma análise de seus respectivos intervalos de credibilidade.

A Figura 31 mostram os gráficos de $c_{t(r, m)}$ versus $t(r, m), r=1, \ldots, n$ para diferentes modelos da série gerada para os meses de Janeiro a Dezembro. 
Janeiro

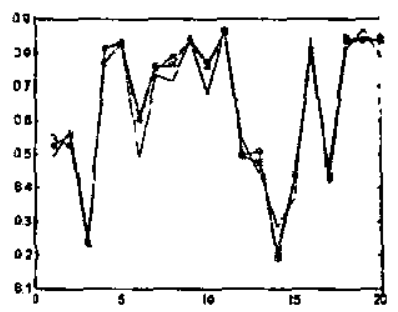

Abril

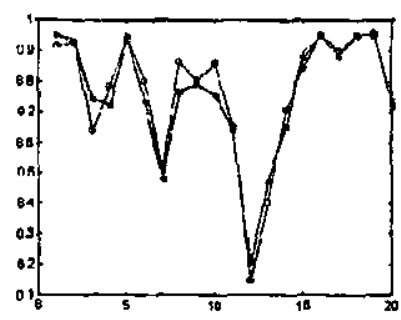

Julho

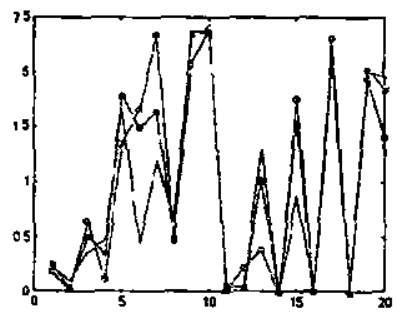

Outubro

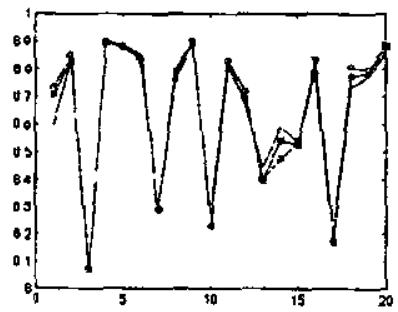

Fevereiro
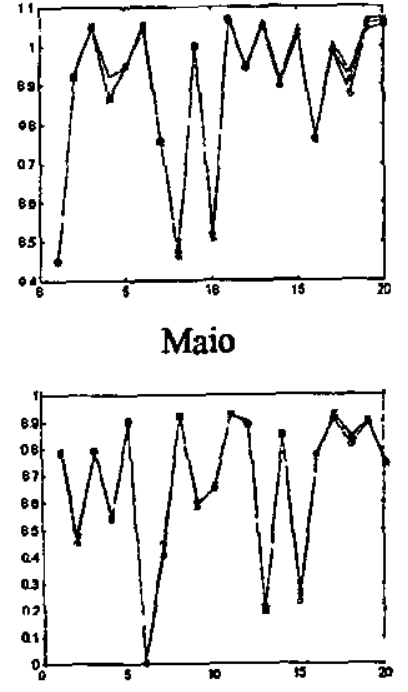

Agosto

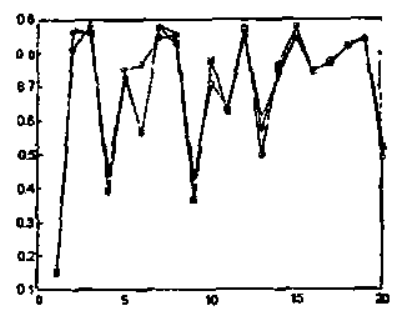

Novembro

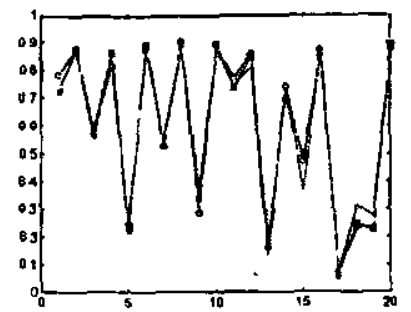

Março

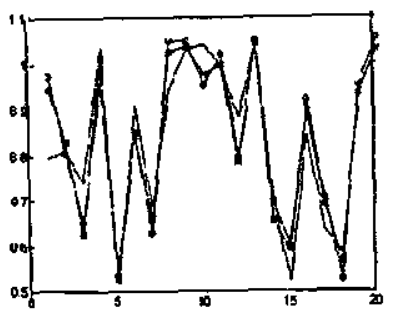

Junho

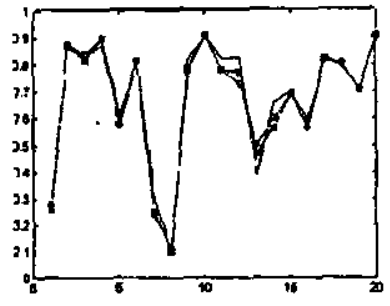

Setembro

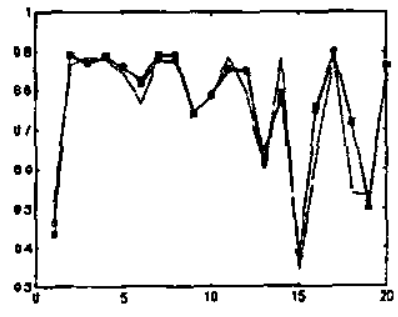

Dezembro

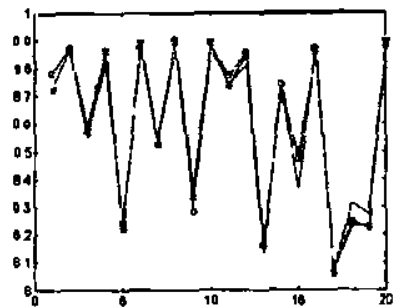

Figura 31. $c_{t r, m)} \times t(r, m), r=1, \ldots, n$ para diferentes modelos para os meses de Janeiro a Dezembro. 
Utilizando as amostras geradas das densidades condicionais de $\phi_{m}$ e $\tau_{m}$, pelos algoritmos Amostrador de Gibbs e Metropolis-Hastings, construímos os gráficos das densidades a posteriori marginais para os meses de Janeiro a Dezembro, ver Figuras 32 a 37 respectivamente.

As densidades a posteriori marginais para os parâmetros $\phi_{1, m}$, e $\tau_{m}$, quando consideramos uma priori Não-Informativa são mostrados pelos histogramas nas Figuras 32 e 33 respectivamente.

Os histogramas das densidades a posteriori marginais para os parâmetros $\phi_{1, m} \mathrm{e}$ $\tau_{m}$, quando consideramos uma priori Normal-Gama para a série gerada são mostrados nas Figuras 34 e 35 respectivamente.

Nas Figuras 36 e 37 respectivamente, apresentamos os histogramas estimado por simulação para os parâmetros $\phi_{1, m}$ e $\tau_{m}$, quando consideramos o produto das priori $t$-Student e Gama para a série gerada. 
Janeiro
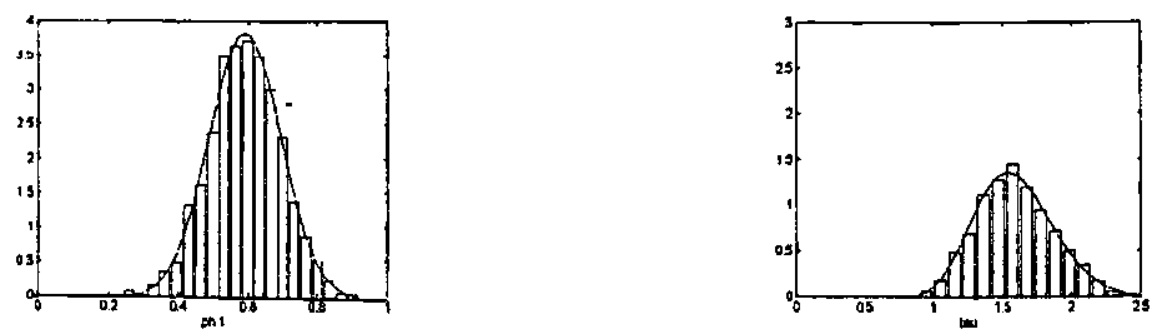

Fevereiro
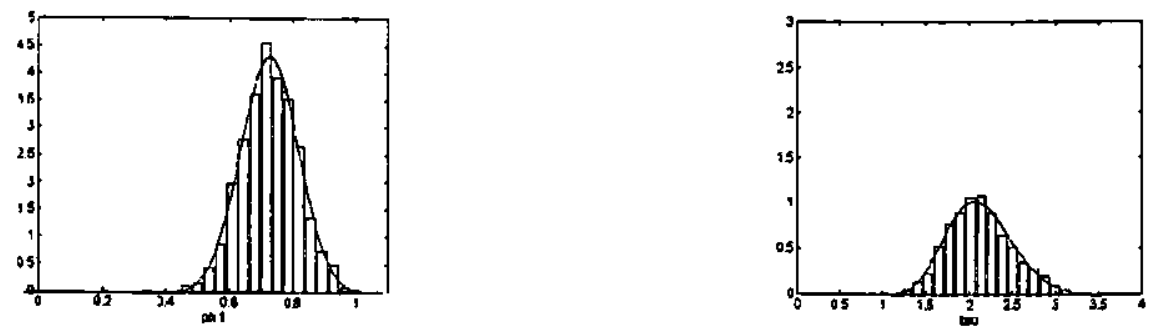

Março
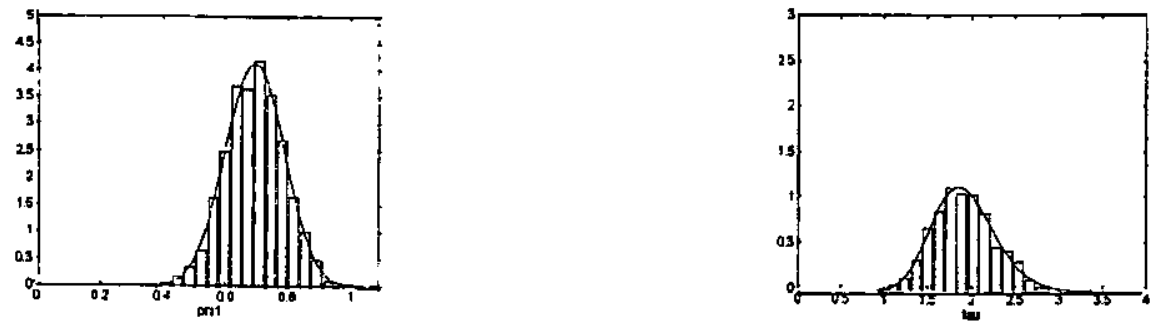

Abril
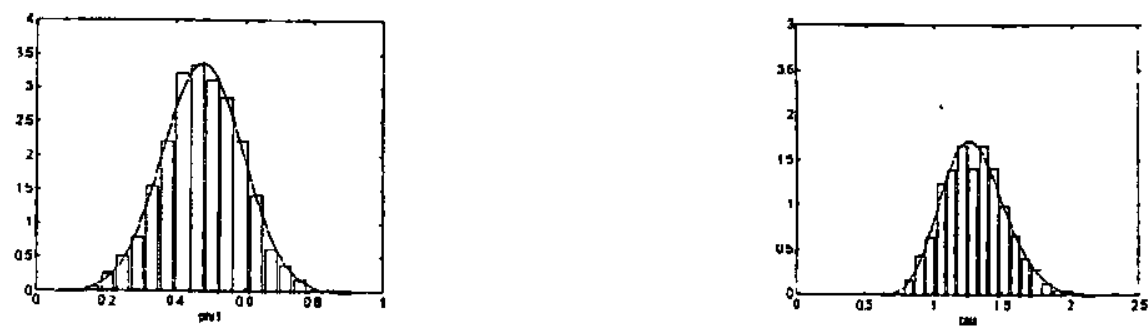

Maio
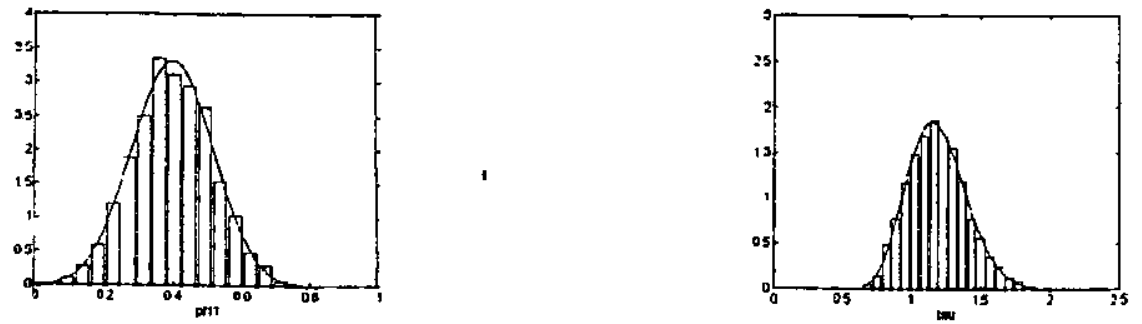

Junho
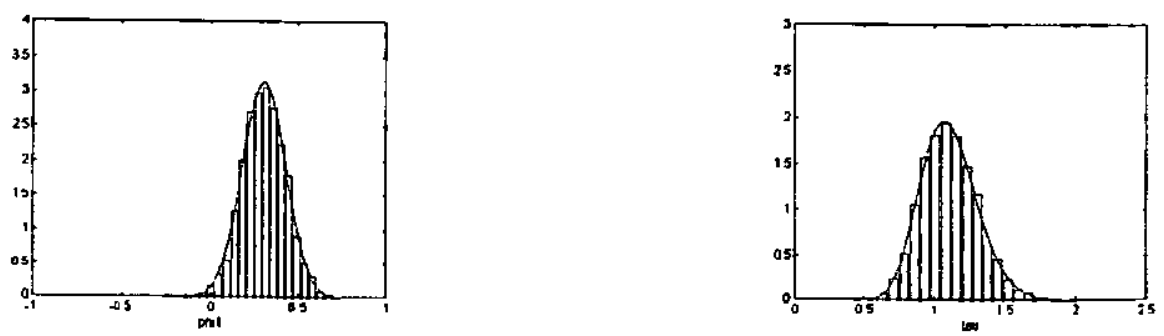

Figura 32. Densidades a Posteriori Marginais para a série gerada considerando a priori Não-Informativa para os meses de Janeiro a Junho respectivamente. 
Julho
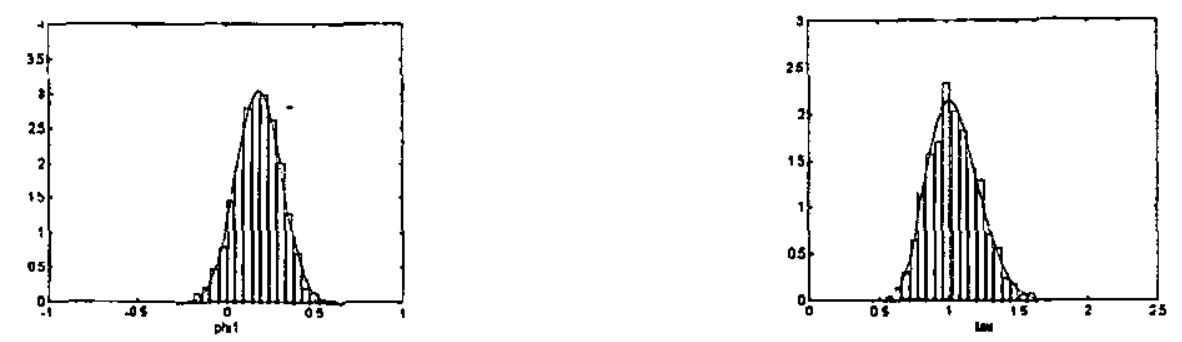

Agosto
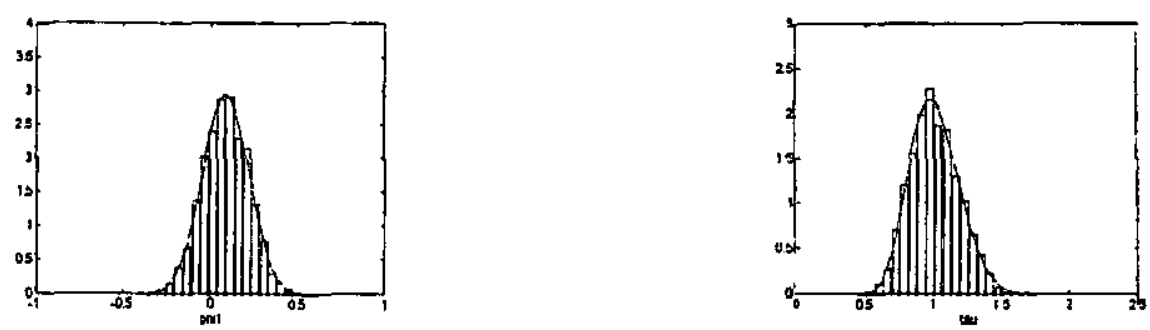

Setembro
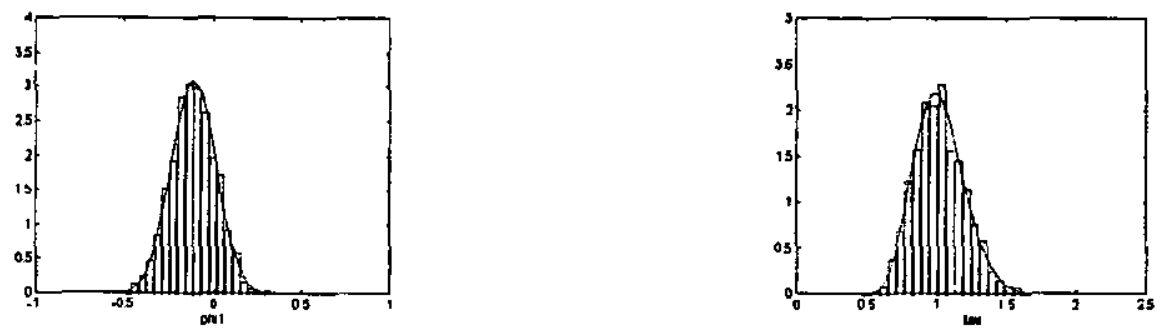

Outubro
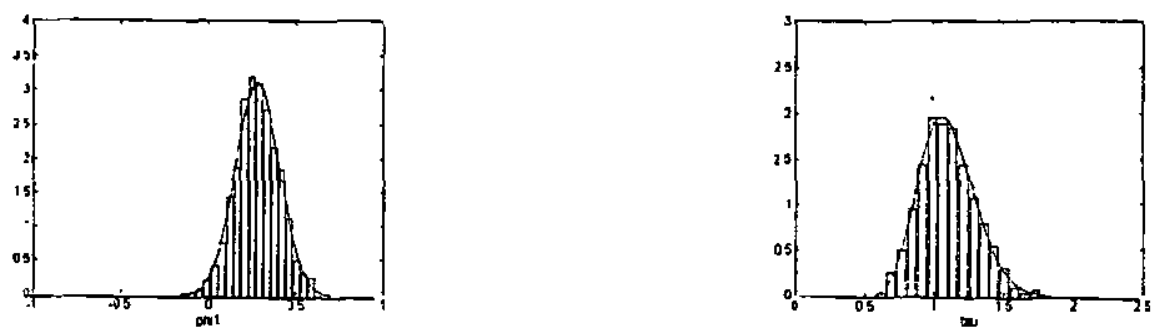

Novembro
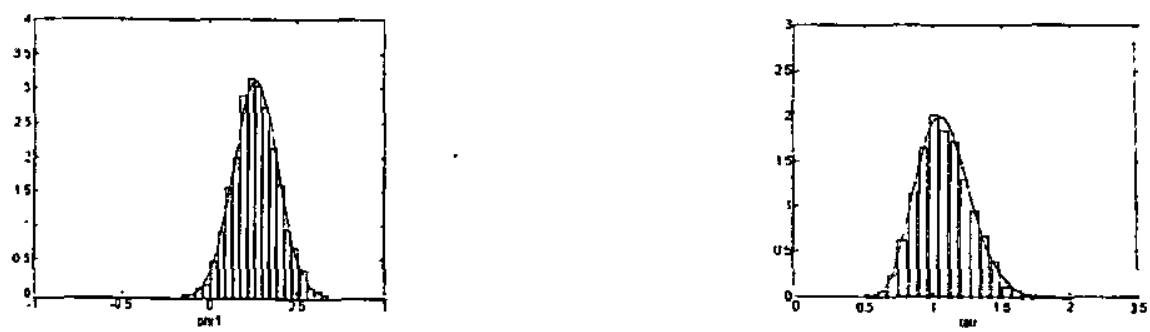

Dezembro
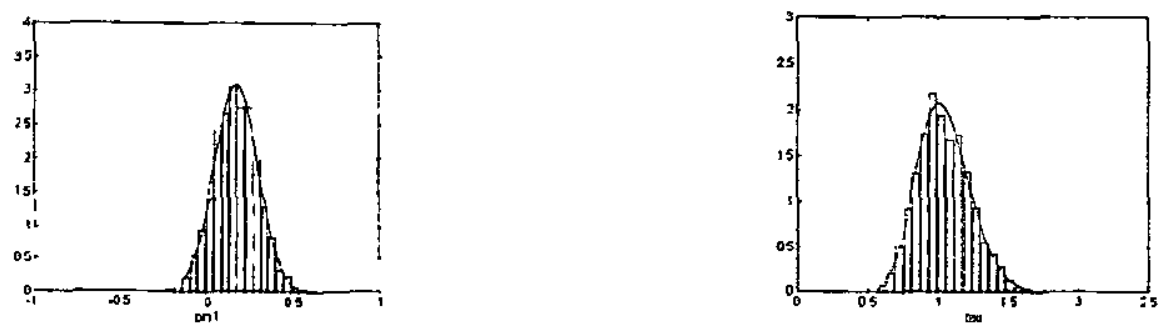

Figura 33. Densidades a Posteriori Marginais para a série gerada considerando a priori Não - Informativa para os meses de Julho a Dezembro respectivamente. 
Janeiro
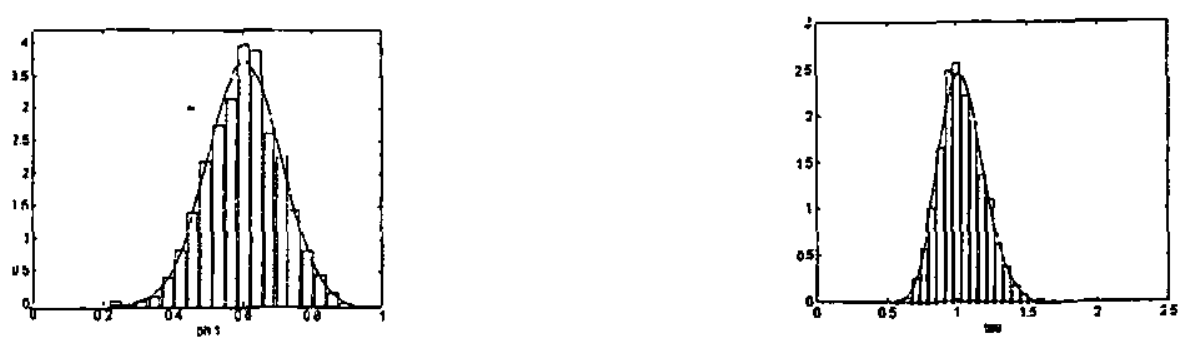

Fevereiro
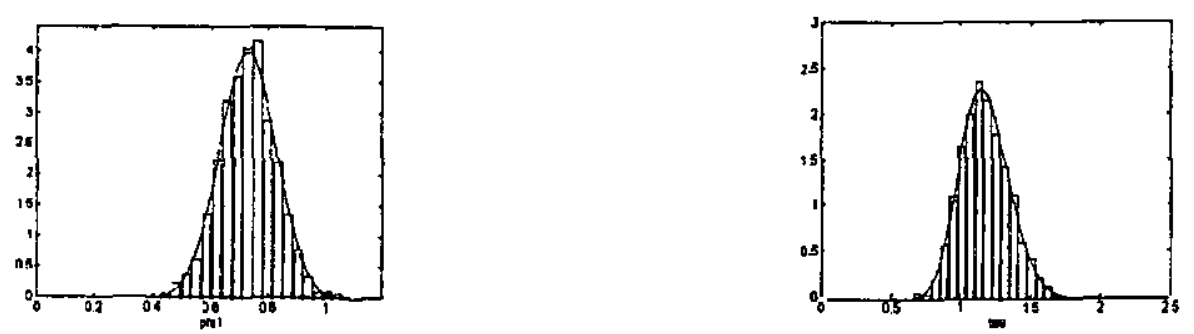

Março
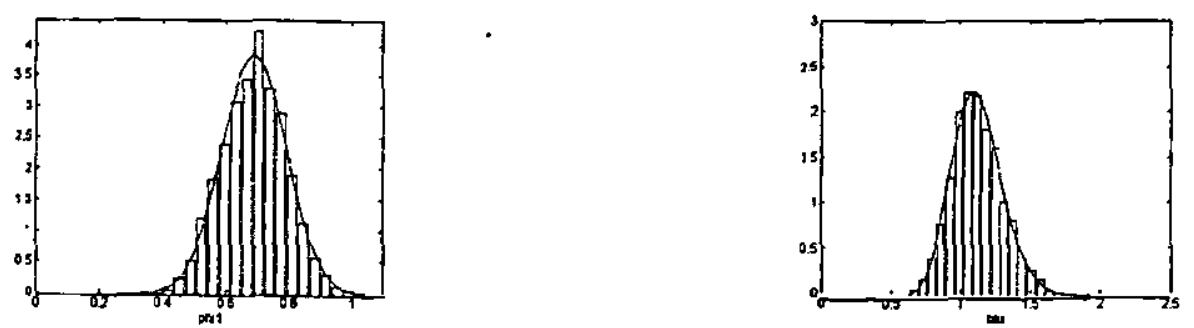

Abril
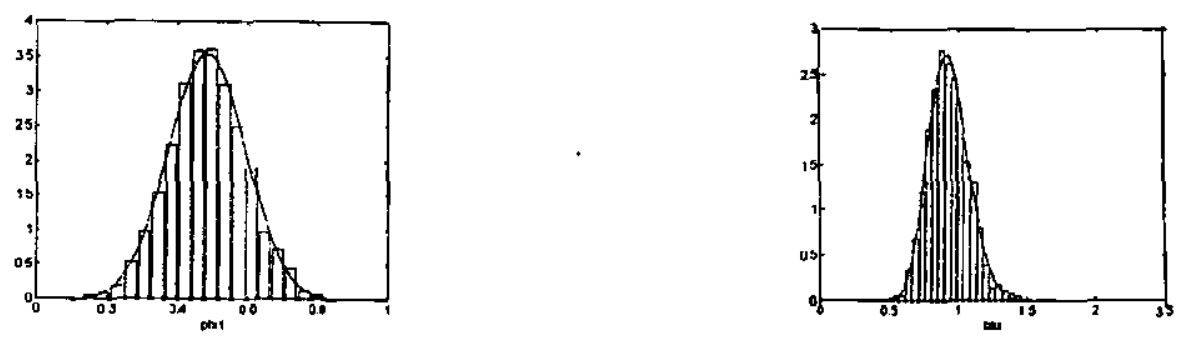

Maio
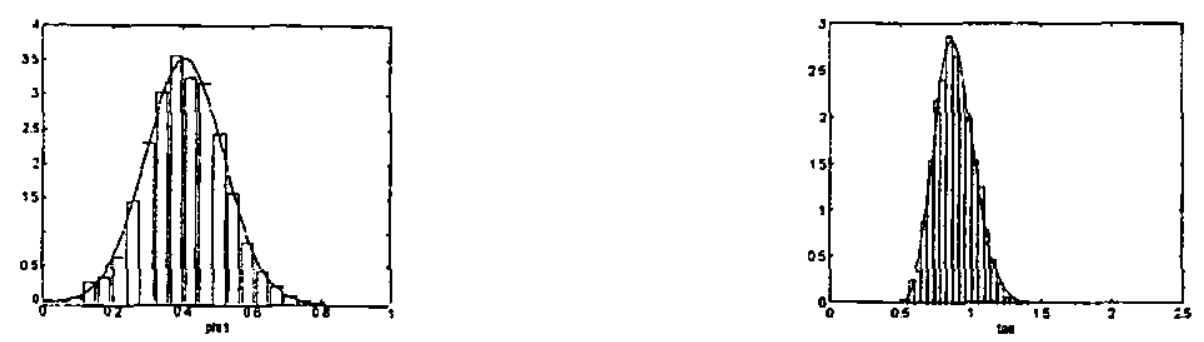

Junho
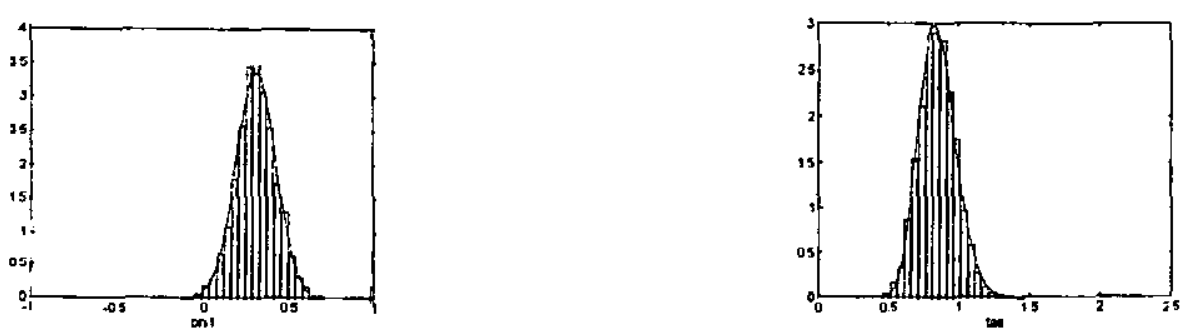

Figura 34. Densidades a Posteriori Marginais para a série gerada considerando a priori Normal-Gama para os meses de Janeiro a Junho respectivamente. 
Julho
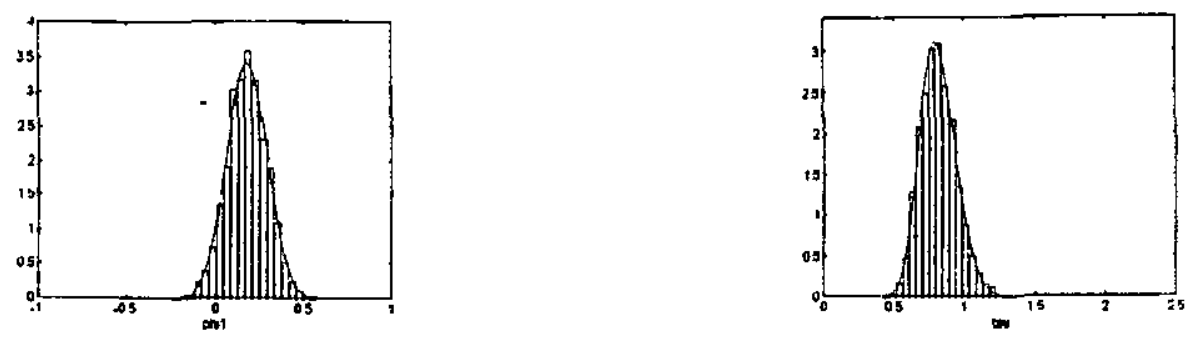

Agosto
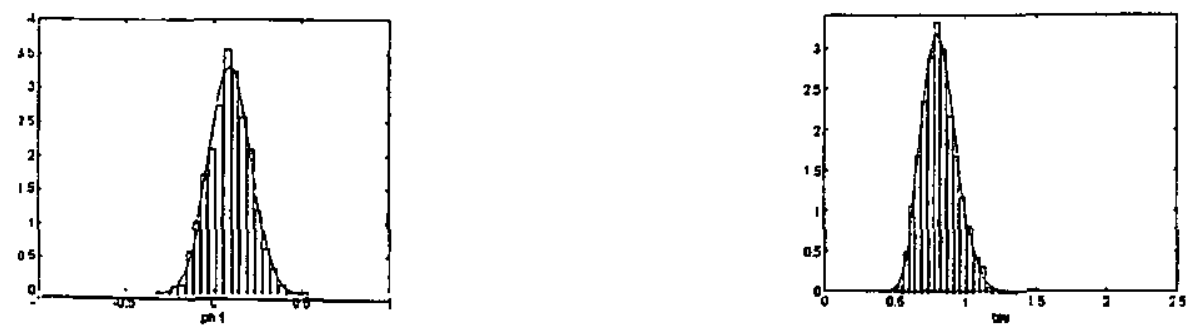

Setembro
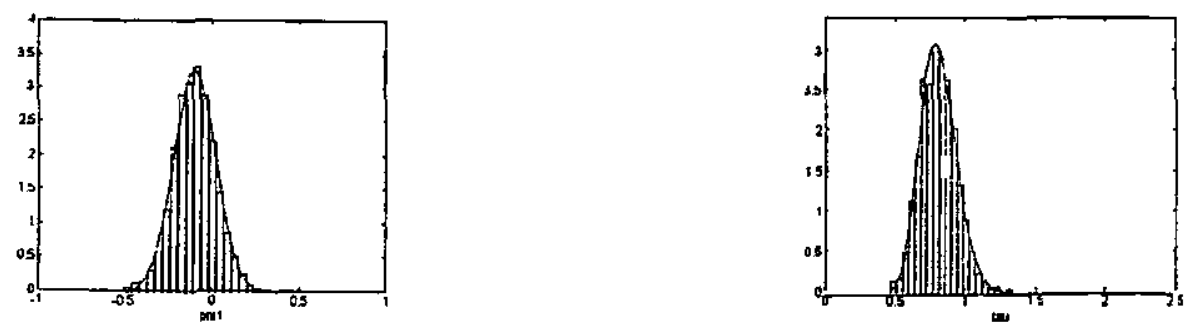

Outubro
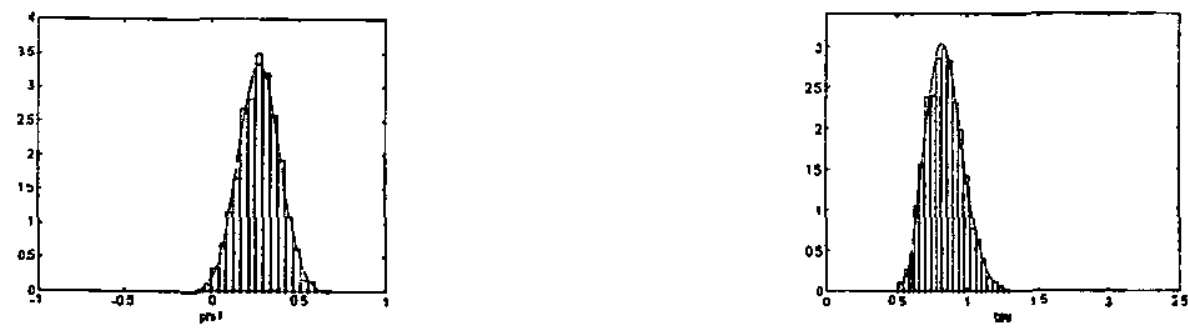

Novembro
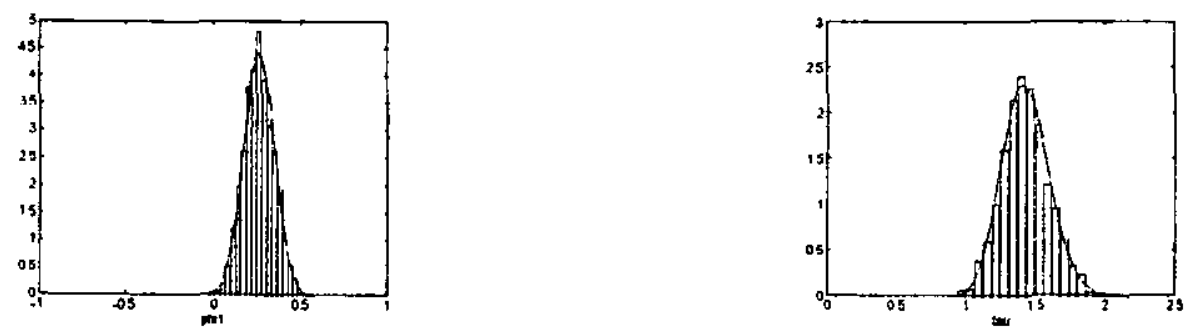

Dezembro
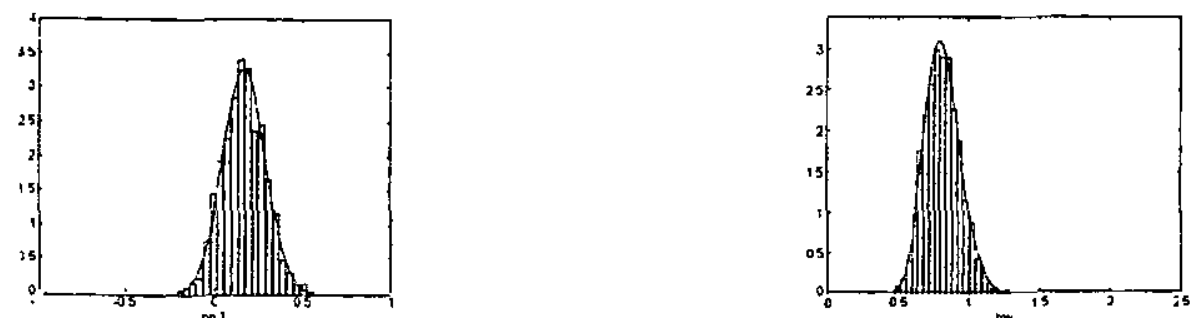

Figura 35. Densidades a Posteriori Marginais para a série gerada considerando a priori Normal-Gama para os meses de Julho a Dezembro respectivamente. 
Janeiro
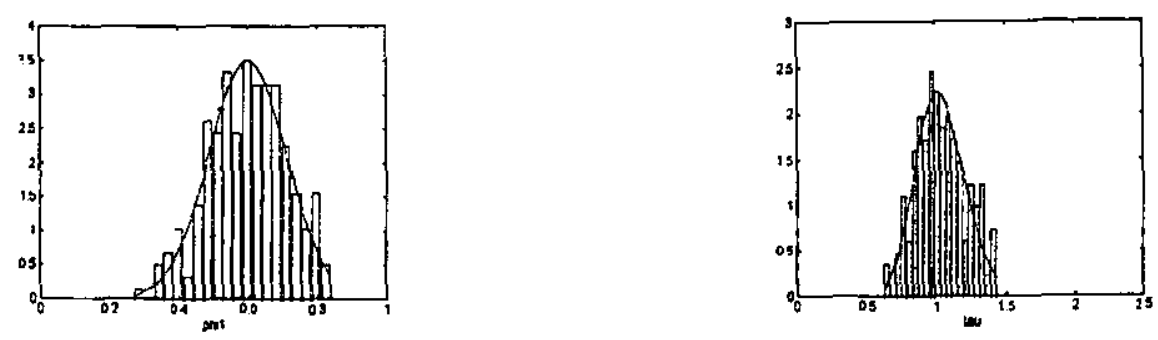

Fevereiro
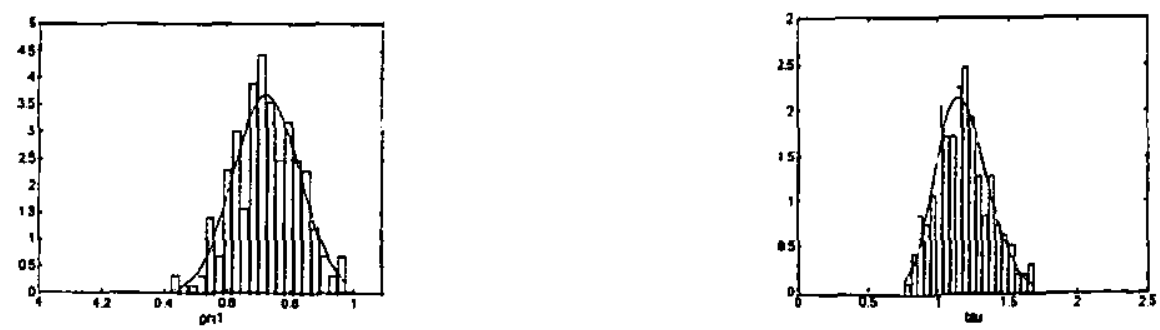

Março
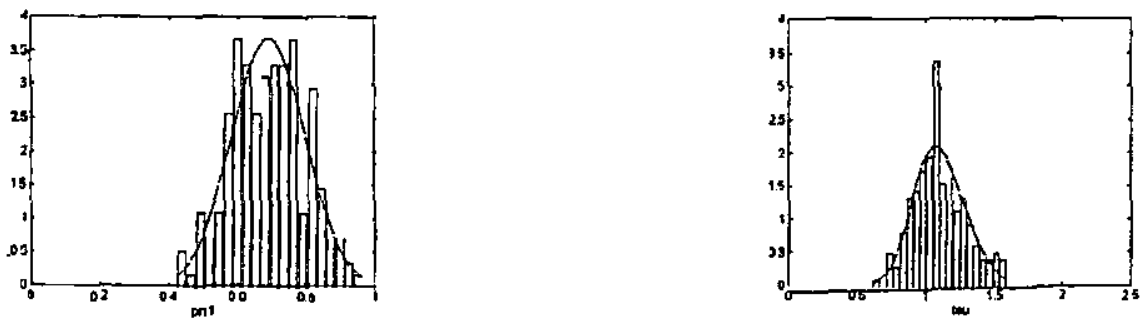

Abril
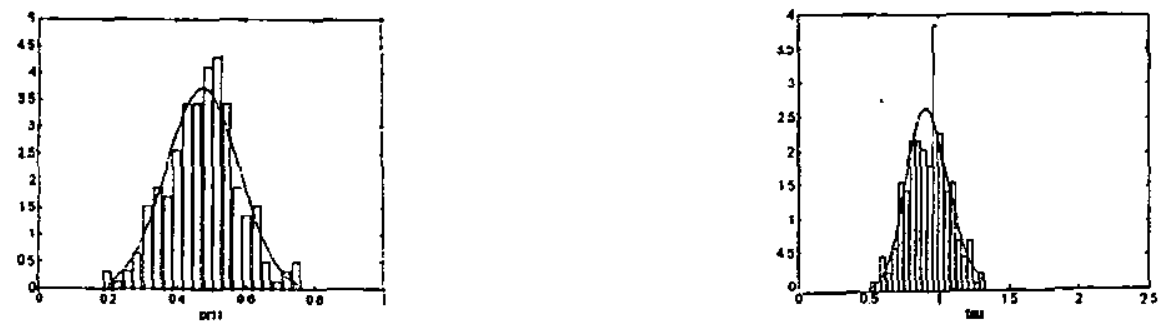

Maio
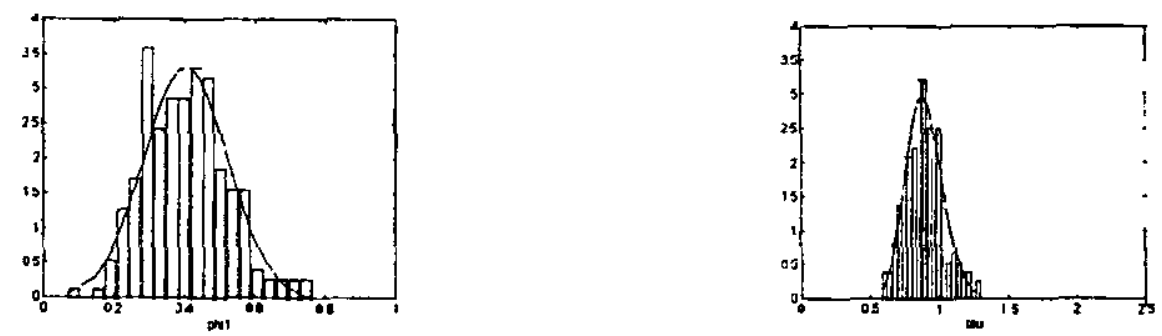

Junho
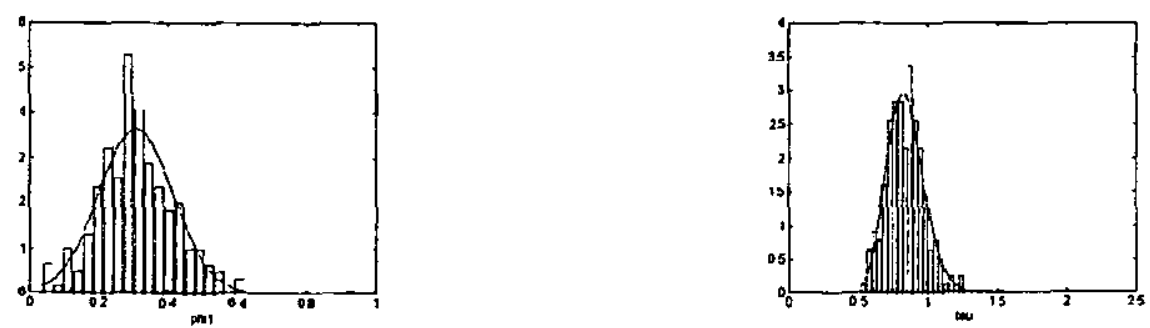

Figura 36. Densidades a Posteriori Marginais para a série gerada considerando a priori t-Student e Gama para os meses de Janeiro a Junho respectivamente. 
Julho
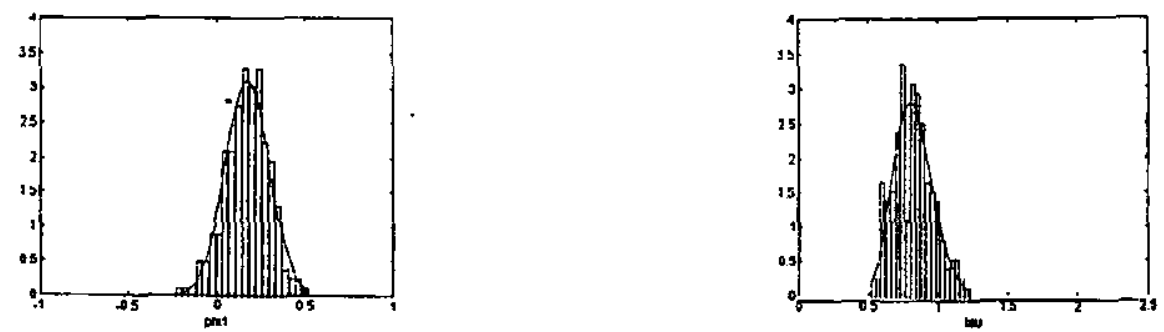

Agosto
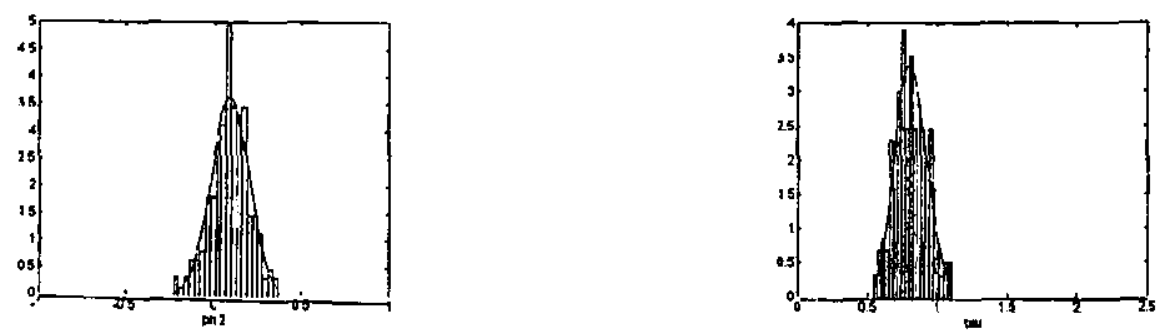

Setembro
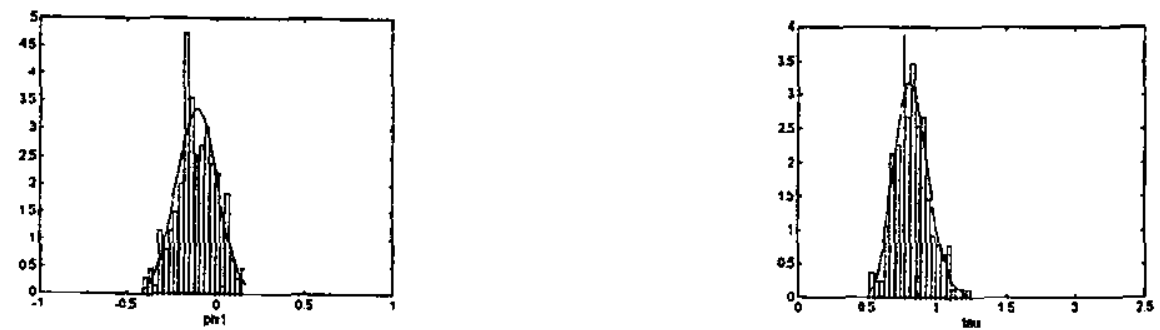

Outubro
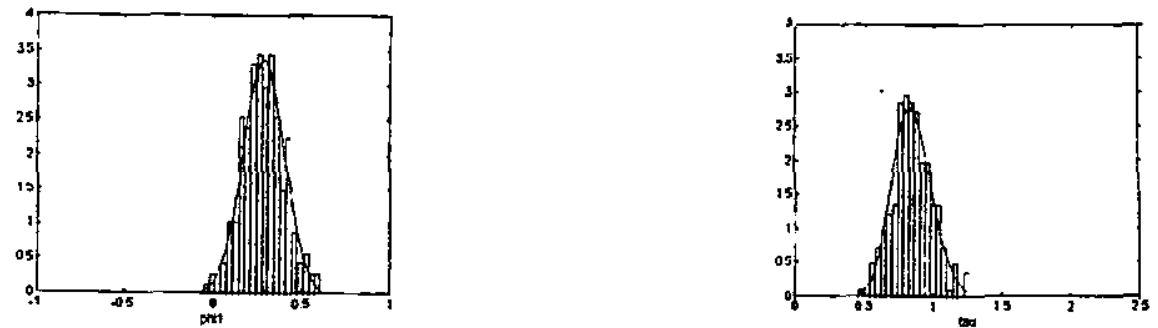

Novembro
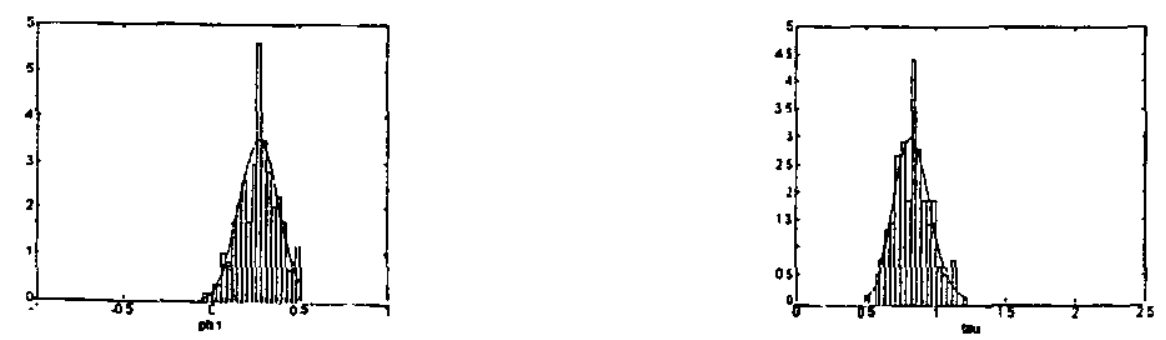

Dezembro
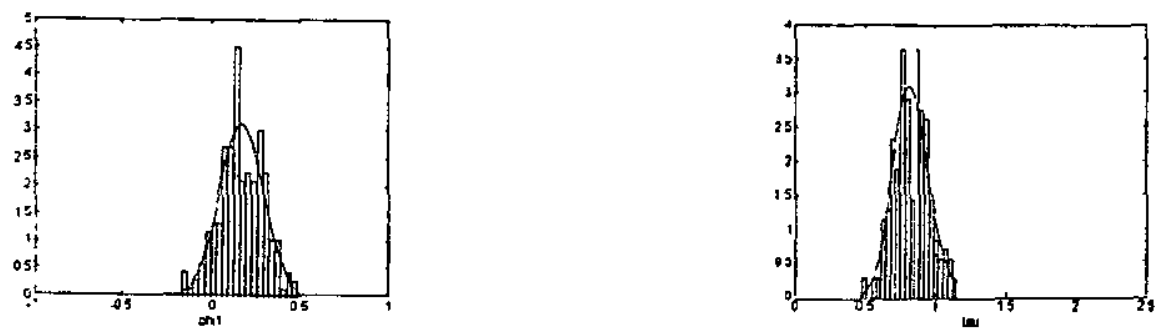

Figura 37. Densidades a Posteriori Marginais para a série gerada considerando a priori t-Student e Gama para os meses de Julho a Dezembro respectivamente. 
No caso do produto das priori $t$-Student e Gama, onde utilizamos o algoritmo de Metropolis-Hastings, verificamos a independência dos dados construíndo o gráfico da correlação dos 6000 pontos amostrais de $\phi_{1, m}$ e $\tau_{m}$, ver Figura 38 . Devido a alta correlação entre os dados da amostra é necessário considerarmos a seleção espaçada, ver Figura 39.

Apresentamos as correlações do mês de Setembro somente devido a semelhança para os outros meses.

A convergência dos dados da série gerada são apresentados nas Figuras 40, 41 e 42 respectivamente.

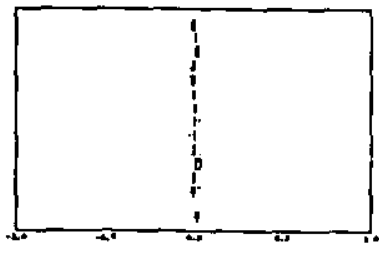

$\tau_{9}$

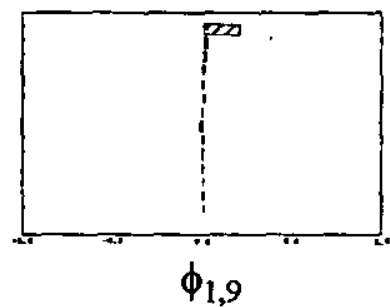

$\phi_{1,9}$

Figura 38. Correlação da amostra gerada pelo amostrador de Gibbs com Metropolis de $\tau_{m}$, e $\phi_{1, m}$ para o mês de Setembro considerando os 3000 pontos finais da seqüência.

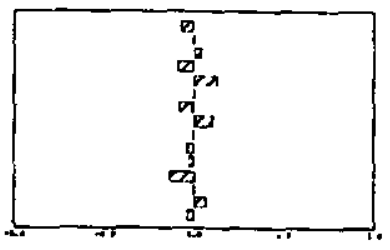

$\tau_{9}$

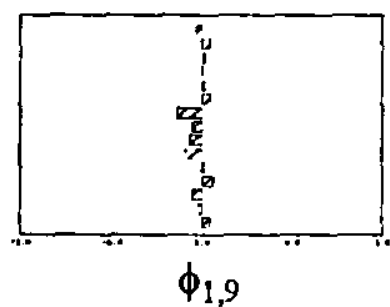

$\phi_{1,9}$

Figura 39. Correlação da amostra de $\tau_{\mathrm{m}}$, e $\phi_{1, \mathrm{~m}}$ para o mês de Setembro, desprezando os 3000 valores iniciais da última seqüência e selecionando-se os 200 pontos restantes de $15 \mathrm{em} 15$. 
Janeiro
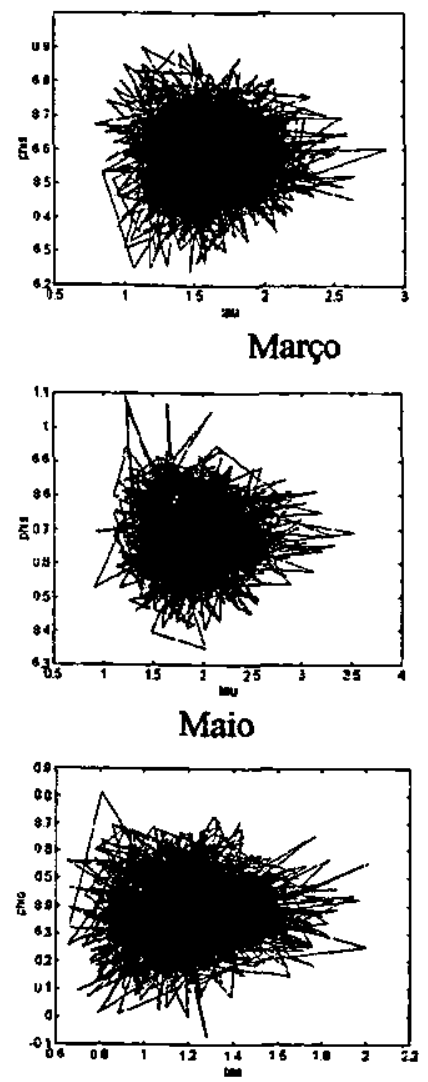

Julho

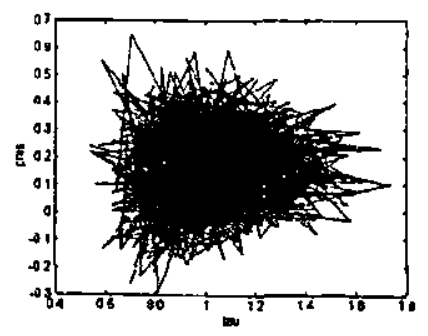

Setembro

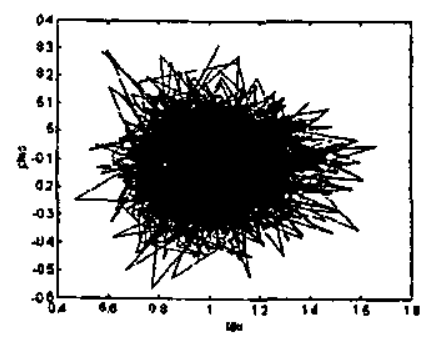

Novembro

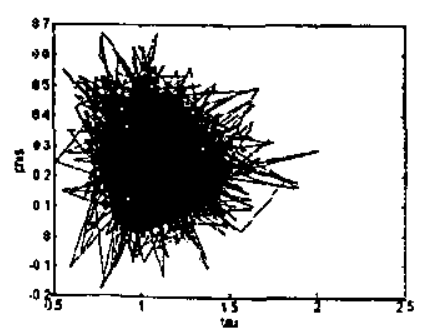

Fevereiro

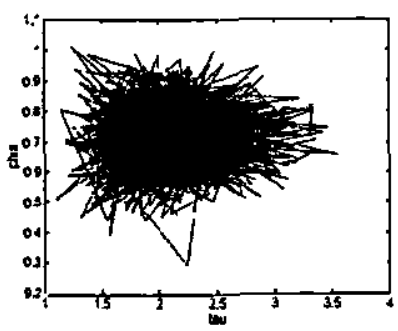

Abril
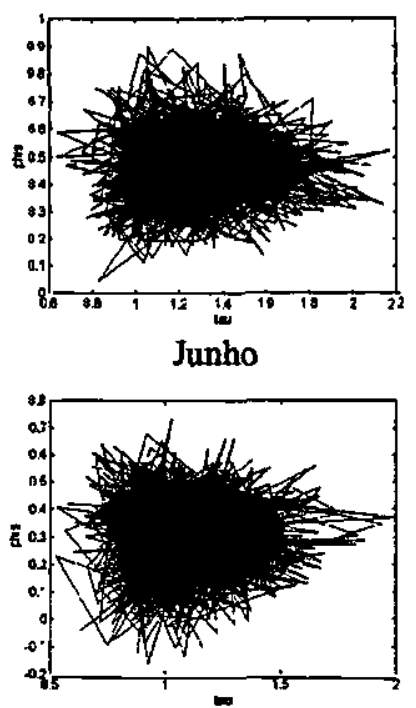

Agosto

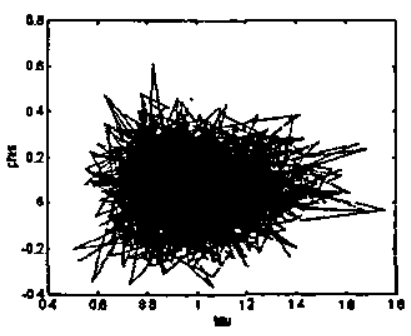

Outubro

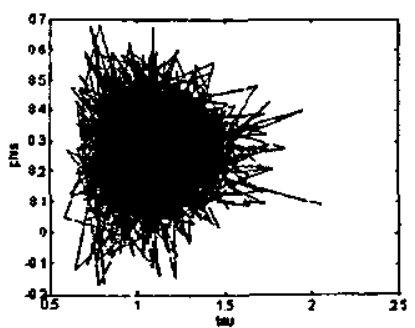

Dezembro

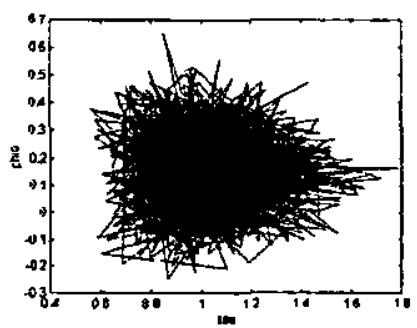

Figura 40. Gráfico da Convergência para a série gerada considerando a priori Não - Informativa para os meses de Janeiro a Dezembro respectivamente. 
Janeiro
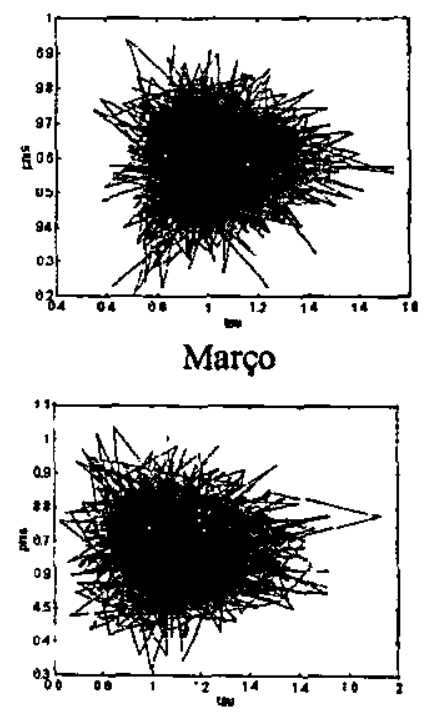

Maio

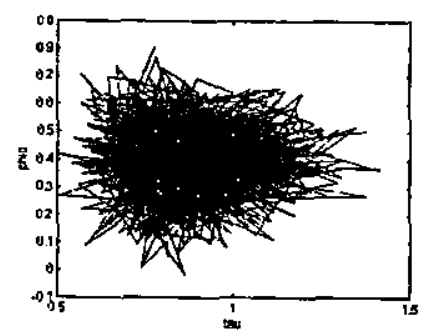

Julho

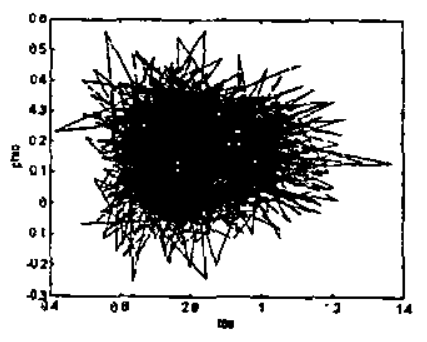

Setembro
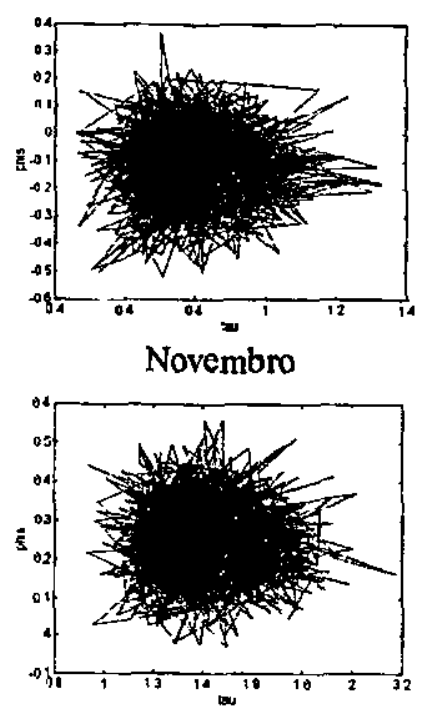

Fevereiro

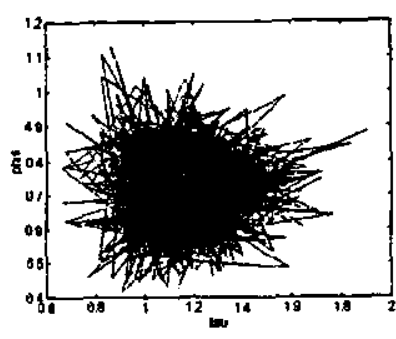

Abril

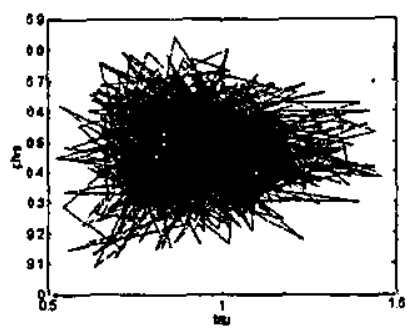

Junho

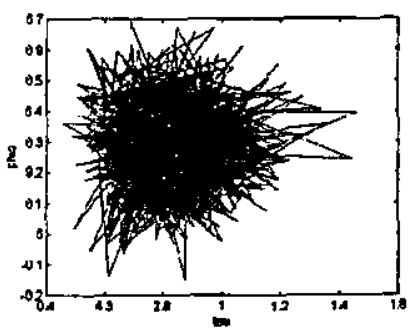

Agosto

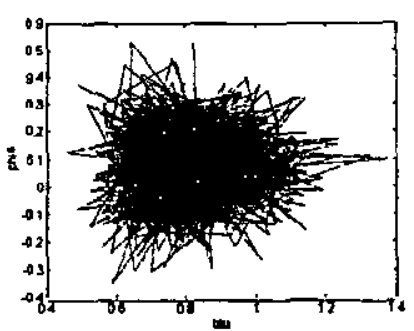

Outubro

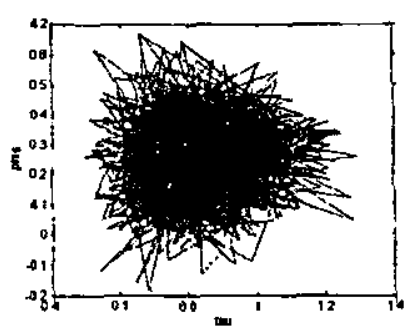

Dezembro

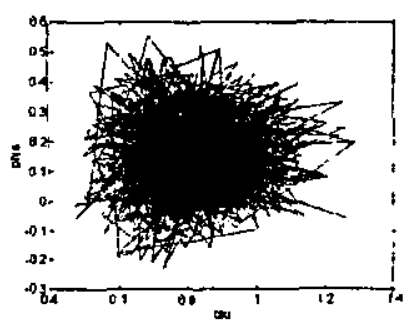

Figura 41. Convergência para a série gerada considerando a priori Normal-Gama para os meses de Janeiro a Dezembro respectivamente. 
Janeiro
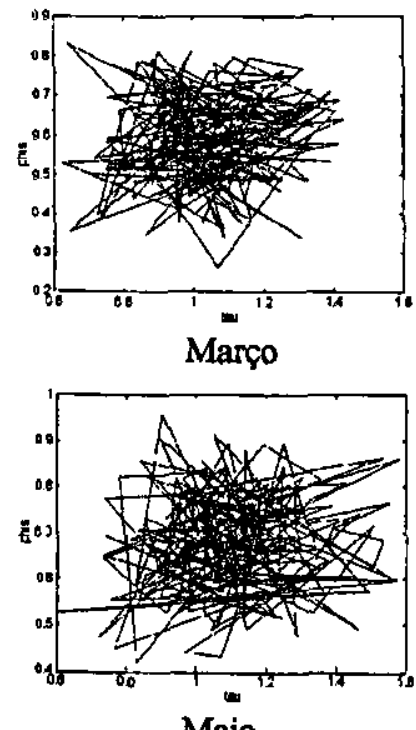

Maio

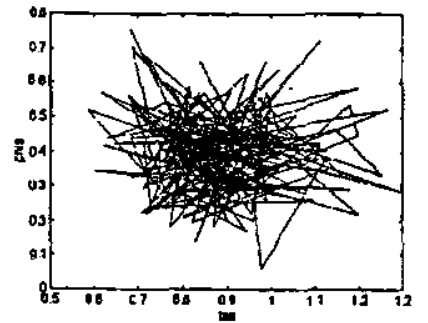

Julho

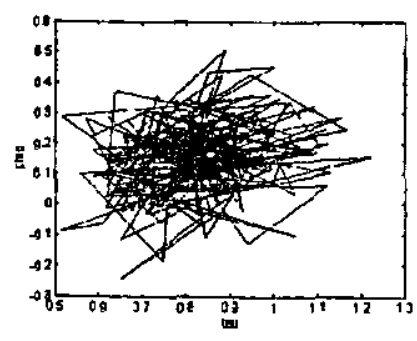

Setembro

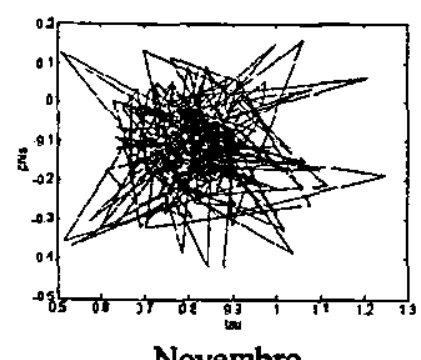

Novembro

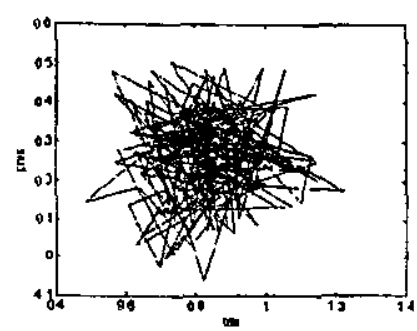

Fevereiro

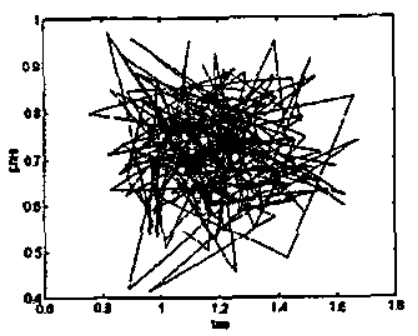

Abril

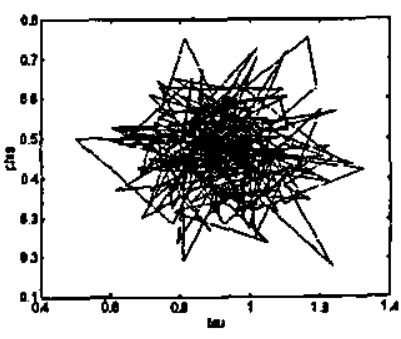

Junho

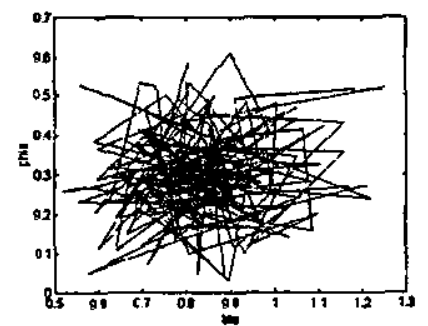

Agosto

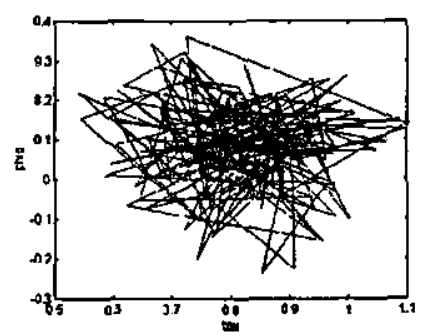

Outubro

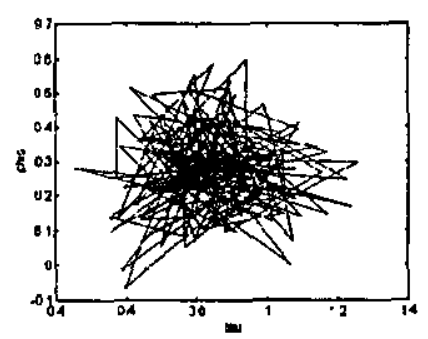

Dezembro

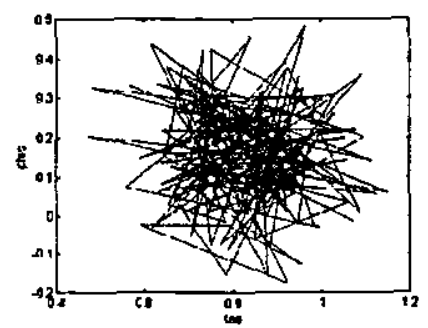

Figura 42. Convergência para a série gerada considerando a priori t-Student e Gama para os meses de Janeiro a Dezembro respectivamente. 
Os resultados dos estimadores dos parâmetros para os meses de Janeiro a Dezembro, considerando as três densidades a priori analisadas neste trabalho são apresentadas na Tabelas 38 a 49.

Tabela 38. Valores Estimados dos parâmetros para o mês de Janeiro

\begin{tabular}{l|rrrrrr}
\hline & $\tau_{m}$ & $D P$ & $R$ & $\phi_{1 m}$ & $D P$ & $R$ \\
\hline EMV & 1,5794 & 0,2908 & & 0,5907 & 0,1039 & \\
PNI(EX) & 1,5794 & 0,2908 & & 0,5907 & 0,1005 & \\
PNI(GS) & 1,5876 & 0,2918 & 0,9997 & 0,5881 & 0,1035 & 1,0032 \\
PNG(EX) & 1,0181 & 0,1630 & & 0,5986 & 0,1061 & \\
PNG(GS) & 1,0286 & 0,1636 & 0,9995 & 0,6003 & 0,1065 & 1,0007 \\
PTSG(MH) & 1,0323 & 0,1779 & 0,9964 & 0,5966 & 0,1131 & 0,9985 \\
\hline
\end{tabular}

Tabela 39. Valores Estimados dos parâmetros para o mês de Fevereiro

\begin{tabular}{l|cccccc}
\hline & $\tau_{m}$ & $D P$ & $R$ & $\phi_{l, m}$ & $D P$ & $R$ \\
\hline EMV & 2,1192 & 0,3902 & & 0,7267 & 0,0894 & \\
PNI(EX) & 2,1192 & 0,3902 & & 0,7267 & 0,0865 & \\
PNI(GS) & 2,1282 & 0,3894 & 0,9995 & 0,7242 & 0,0919 & 0,9997 \\
PNG(EX) & 1,1498 & 0,1841 & & 0,7260 & 0,0997 & \\
PNG(GS) & 1,1674 & 0,1755 & 0,9996 & 0,7265 & 0,0994 & 1,0011 \\
PTSG(MH) & 1,1696 & 0,1883 & 1,0203 & 0,7230 & 0,1070 & 1,0093 \\
\hline
\end{tabular}

Tabela 40. Valores Estimados dos parâmetros para o mês de Março

\begin{tabular}{l|cccccc}
\hline & $\tau_{m}$ & $D P$ & $R$ & $\phi_{1, m}$ & $D P$ & $R$ \\
\hline EMV & 1,9063 & 0,3510 & & 0,6895 & 0,0943 & \\
PNI(EX) & 1,9063 & 0,3510 & & 0,6895 & 0,0912 & \\
PNI(GS) & 1,9152 & 0,3573 & 1,0026 & 0,6912 & 0,0958 & 1,0002 \\
PNG(EX) & 1,0999 & 0,1761 & & 0,6881 & 0,1019 & \\
PNG(GS) & 1,1145 & 0,1808 & 1,0002 & 0,6859 & 0,1030 & 1,0007 \\
PTSG(MH) & 1,0967 & 0,1915 & 0,9996 & 0,6861 & 0,1075 & 1,0693 \\
\hline
\end{tabular}

Tabela 41. Valores Estimados dos parâmetros para o mês de Abril

\begin{tabular}{l|cccccc}
\hline & $\tau_{m 0}$ & $D P$ & $R$ & $\phi_{l, m}$ & $D P$ & $R$ \\
\hline EMV & 1,2899 & 0,2375 & & 0,4741 & 0,1146 & \\
PNI(EX) & 1,2899 & 0,2375 & & 0,4741 & 0,1109 & \\
PNI(GS) & 1,2927 & 0,2326 & 1,0014 & 0,4754 & 0,1176 & 1,0013 \\
PNG(EX) & 0,9155 & 0,1466 & & 0,4803 & 0,1117 & \\
PNG(GS) & 0,9270 & 0,1482 & 0,9996 & 0,4794 & 0,1118 & 0,9996 \\
PTSG(MH) & 0,9239 & 0,1499 & 0,9987 & 0,4736 & 0,1058 & 1,0081 \\
\hline
\end{tabular}


Tabela 42. Valores Estimados dos parâmetros para o mês de Maio

\begin{tabular}{l|cccccc}
\hline & $\tau_{m}$ & $D P$ & $R$ & $\phi_{l, m}$ & $D P$ & $R$ \\
\hline EMV & 1,1841 & 0,2180 & & 0,3943 & 0,1196 & \\
PNI(EX) & 1,1841 & 0,2180 & - & 0,3943 & 0,1157 & \\
PNI(GS) & 1,1849 & 0,2175 & 1,0001 & 0,3928 & 0,1192 & 0,9998 \\
PNG(EX) & 0,8728 & 0,1398 & & 0,4001 & 0,1144 & \\
PNG(GS) & 0,8841 & 0,1414 & 1,0005 & 0,4033 & 0,1124 & 1,0008 \\
PTSG(MH) & 0,8876 & 0,1360 & 0,9988 & 0,4023 & 0,1197 & 0,9981 \\
\hline
\end{tabular}

Tabela 43. Valores Estimados dos parâmetros para o mês de Junho

\begin{tabular}{l|cccccc}
\hline & $\tau_{m}$ & $D P$ & $R$ & $\phi_{l, m}$ & $D P$ & $R$ \\
\hline EMV & 1,1013 & 0,2028 & & 0,3033 & 0,1241 & \\
PNI(EX) & 1,1013 & 0,2028 & & 0,3033 & 0,1200 & \\
PNI(GS) & 1,1007 & 0,2036 & 1,0008 & 0,3018 & 0,1259 & 1,0028 \\
PNG(EX) & 0,8339 & 0,1335 & & 0,3016 & 0,1170 & \\
PNG(GS) & 0,8425 & 0,1336 & 1,0006 & 0,2998 & 0,1177 & 1,0003 \\
PTSG(MH) & 0,8315 & 0,1360 & 0,9969 & 0,3050 & 0,1086 & 1,0294 \\
\hline
\end{tabular}

Tabela 44. Valores Estimados dos parâmetros para o mês de Julho

\begin{tabular}{l|cccccc}
\hline & $\tau_{m}$ & $D P$ & $R$ & $\phi_{l, m}$ & $D P$ & $R$ \\
\hline EMV & 1,0320 & 0,1900 & & 0,1761 & 0,1282 & \\
PNI(EX) & 1,0320 & 0,1900 & & 0,1761 & 0,1240 & \\
PNI(GS) & 1,0338 & 0,1865 & 1,0000 & 0,1784 & 0,1297 & 0,9997 \\
PNG(EX) & 0,8033 & 0,1286 & & 0,1777 & 0,1191 & \\
PNG(GS) & 0,8118 & 0,1291 & 1,0019 & 0,1759 & 0,1157 & 1,0009 \\
PTSG(MH) & 0,8185 & 0,1431 & 1,0110 & 0,1680 & 0,1267 & 1,0172 \\
\hline
\end{tabular}

Tabela 45. Valores Estimados dos parâmetros para o mês de Agosto

\begin{tabular}{l|cccccc}
\hline & $\tau_{m}$ & $D P$ & $R$ & $\phi_{l, m}$ & $D P$ & $R$ \\
\hline EMV & 1,0071 & 0,1854 & & 0,0837 & 0,1297 & \\
PNI(EX) & 1,0071 & 0,1854 & & 0,0854 & 0,1255 & \\
PNI(GS) & 1,0087 & 0,1852 & 0,9996 & 0,0810 & 0,1350 & 1,0002 \\
PNG(EX) & 0,8015 & 0,1283 & & 0,0875 & 0,1192 & \\
PNG(GS) & 0,8128 & 0,1267 & 1,0037 & 0,0867 & 0,1195 & 1,0015 \\
PTSG(MH) & 0,8104 & 0,1169 & 0,9954 & 0,0903 & 0,1091 & 1,0032 \\
\hline
\end{tabular}


Tabela 46. Valores Estimados dos parâmetros para o mês de Setembro

\begin{tabular}{l|cccccc}
\hline & $\tau_{m}$ & $D P$ & $R$ & $\phi_{l, m}$ & $D P$ & $R$ \\
\hline EMV & 1,0130 & 0,1868 & & $-0,1133$ & 0,1294 & \\
PNI(EX) & 1,0130 & 0,1865 & - & $-0,1133$ & 0,1251 & \\
PNI(GS) & 1,0163 & 0,1839 & 1,0001 & $-0,1139$ & 0,1284 & 0,9995 \\
PNG(EX) & 0,7949 & 0,1273 & & $-0,1067$ & 0,1205 & \\
PNG(GS) & 0,8049 & 0,1297 & 1,0018 & $-0,1042$ & 0,1218 & 1,0021 \\
PTSG(MH) & 0,8156 & 0,1268 & 1,0308 & $-0,1114$ & 0,1171 & 1,0239 \\
\hline
\end{tabular}

Tabela 47. Valores Estimados dos parâmetros para o mês de Outubro

\begin{tabular}{l|cccccc}
\hline & $\tau_{m}$ & $D P$ & $R$ & $\phi_{l, m}$ & $D P$ & $R$ \\
\hline EMV & 1,0804 & 0,1989 & & 0,2727 & 0,1253 & \\
PNI(EX) & 1,0804 & 0,1989 & & 0,2727 & 0,1211 & \\
PNI(GS) & 1,0884 & 0,2050 & 1,0007 & 0,2748 & 0,1271 & 1,0030 \\
PNG(EX) & 0,8255 & 0,1322 & & 0,2699 & 0,1176 & \\
PNG(GS) & 0,8371 & 0,1315 & 0,9996 & 0,2680 & 0,1186 & 0,9997 \\
PTSG(MH) & 0,8438 & 0,1432 & 1,0092 & 0,2827 & 0,1175 & 1,0079 \\
\hline
\end{tabular}

Tabela 48. Valores Estimados dos parâmetros para o mês de Novembro

\begin{tabular}{l|cccccc}
\hline & $\tau_{m}$ & $D P$ & $R$ & $\phi_{l m}$ & $D P$ & $R$ \\
\hline EMV & 1,0720 & 0,1974 & & 0,2591 & 0,1257 & \\
PNI(EX) & 1,0720 & 0,1974 & & 0,2591 & 0,1216 & \\
PNI(GS) & 1,0787 & 0,2003 & 1,0024 & 0,2620 & 0,1265 & 1,0028 \\
PNG(EX) & 1,4180 & 0,1724 & & 0,2572 & 0,0897 & \\
PNG(GS) & 1,4239 & 0,1726 & 1,0027 & 0,2568 & 0,0897 & 1,0002 \\
PTSG(MH) & 0,8301 & 0,1334 & 1,0292 & 0,2679 & 0,1123 & 0,9984 \\
\hline
\end{tabular}

Tabela 49. Valores Estimados dos parâmetros para o mês de Dezembro

\begin{tabular}{l|cccccc}
\hline & $\tau_{m}$ & $D P$ & $R$ & $\phi_{l m}$ & $D P$ & $R$ \\
\hline EMV & 1,0265 & 0,1890 & & 0,1607 & 0,1285 & \\
PNI(EX) & 1,0265 & 0,1890 & & 0,1607 & 0,1243 & \\
PNI(GS) & 1,0365 & 0,1938 & 1,0015 & 0,1592 & 0,1280 & 1,0000 \\
PNG(EX) & 0,8035 & 0,1287 & & 0,1645 & 0,1191 & \\
PNG(GS) & 0,8122 & 0,1287 & 1,0013 & 0,1651 & 0,1207 & 0,9995 \\
PTSG(MH) & 0,8227 & 0,1276 & 1,0143 & 0,1678 & 0,1265 & 1,0138 \\
\hline
\end{tabular}


O intervalo de credibilidade para o produto das priori $t$-Student e Gama são apresentados na Tabela 50.

Tabela 50. Intervalo de Credibilidade para os parâmetros $\tau_{\mathrm{m}}, \mathrm{e} \phi_{1, \mathrm{~m}}$ (Priori $t$-Student e Gama- Janeiro a Dezembro)

\begin{tabular}{l|cc}
\hline més $m$ & $\tau_{\mathrm{m}}$ & $\phi_{\mathrm{l}, \mathrm{m}}$ \\
\hline Janeiro $(\mathrm{m}=1)$ & $(0,7152 ; 1,3847)$ & $(0,3594 ; 0,7985)$ \\
Fevereiro $(\mathrm{m}=2)$ & $(0,8252 ; 1,5673)$ & $(0,5095 ; 0,9282)$ \\
Marco $(\mathrm{m}=3)$ & $(0,7374 ; 1,5301)$ & $(0,4782 ; 0,8966)$ \\
Abril $(\mathrm{m}=4)$ & $(0,6098 ; 1,2267)$ & $(0,2587 ; 0,6811)$ \\
Maio $(\mathrm{m}=5)$ & $(0,6268 ; 1,1943)$ & $(0,1907 ; 0,6697)$ \\
Junho $(\mathrm{m}=6)$ & $(0,5864 ; 1,1105)$ & $(0,0757 ; 0,5295)$ \\
Julho $(\mathrm{m}=7)$ & $(0,5832 ; 1,1206)$ & $(-0,1029 ; 0,3795)$ \\
Agosto $(\mathrm{m}=8)$ & $(0,5921 ; 1,0464)$ & $(-0,1483 ; 0,2960)$ \\
Setembro $(\mathrm{m}=9)$ & $(0,5698 ; 1,0761)$ & $(-0,3654 ; 0,1004)$ \\
Outubro $(\mathrm{m}=10)$ & $(0,5689 ; 1,1506)$ & $(0,0582 ; 0,5218)$ \\
Novembro $(\mathrm{m}=11)$ & $(0,5769 ; 1,1256)$ & $(0,0348 ; 0,4899)$ \\
Dezembro $(\mathrm{m}=12)$ & $(0,5857 ; 1,0860)$ & $(-0,0828 ; 0,3977)$ \\
\hline
\end{tabular}

$\mathrm{Na}$ Tabela 50, observamos que os intervalos de credibilidade são siginificativamente diferentes de zero para a maioria dos meses, conferindo com o BIC, que indica para a maioria dos meses ajustar modelos de ordem um.

Nas Figuras 32 a 35 observamos que a forma simétrica da posteriori coincide com a forma esperada teoricamente.

Observamos nas Figuras 36 e 37 que apesar de não conhecermos a distribuição a posteriori, os histogramas também se apresentam de uma forma simétrica.

Nas Tabelas 38 a 49 , podemos observar que os resultados a posteriori para $\phi_{1, m}$ estão bem póximos do verdadeiro valor do parâmetro, ver Tabela 1, quando usamos as densidades a priori não-informativa e as informativas apresentadas neste trabalho. Os resultados a posteriori para $\tau_{m}$, para alguns meses estão se afastando do verdadeiro valor do parâmetro utilizado, isto ocorre principalmente porque a 
definição dos hiperparâmetros não esta coerente ou a distribuição a priori considerada não é informativa o suficiente.

Observamos através de $\mathrm{R}$ (fator de redução de escala) e pelos gráficos das Figuras 40,41 e 42 que a convergência é alcançada para as três densidades a priori.

\subsection{Previsão dos Valores Futuros}

Através da abordagem Bayesiana com o uso da priori conjugada Normal-Gama obtemos a previsão um passo a frente para os meses de Janeiro a Dezembro para as séries analisadas acima. O erro médio percentual também foi calculado (EMP), esses resultados são mostrados respectivamente nas Tabelas 51 a 53 , onde podemos notar o bom desempenho do método bayesiano para fazer previsões usando-se os algoritmos de MCMC.

As Figuras 43,44 e 45 a seguir mostram a previsão para os séries de Furnas, Emborcação e para a série gerada respectivamente e observamos que a previsões acompanham a tendência das séries analisadas. 
Tabela 51. Previsão um passo a frente para a série de Furnas

\begin{tabular}{l|c|c}
\hline Meses & Valor Real & Valor Previsto \\
\hline Janeiro & 1343 & 1719 \\
Fevereiro & 643 & 1652 \\
Março & 1051 & 983 \\
Abril & 695 & 779 \\
Maio & 595 & .676 \\
Junho & 391 & 578 \\
Julho & 403 & 492 \\
Agosto & 379 & 420 \\
Setembro & 435 & 430 \\
Outubro & 432 & 527 \\
Novembro & 378 & 734 \\
Dezembro & 576 & 1241 \\
\hline EMP & 0,44 &
\end{tabular}

Tabela 52. Previsão um passo a frente

\begin{tabular}{l|c|c}
\multicolumn{3}{c}{ para a série de Emborcação } \\
\hline Meses & Valor Real & Valor Previsto \\
\hline Janeiro & 1070 & 923 \\
Fevereiro & 586 & 1045 \\
Março & 548 & 767 \\
Abril & 404 & 579 \\
Maio & 355 & 373 \\
Junho & 261 & 302 \\
Julho & 238 & 238 \\
Agosto & 182 & 189 \\
Setembro & 182 & 161 \\
Outubro & 199 & 208 \\
Novembro & 201 & 355 \\
Dezembro & 236 & 642 \\
\hline EMP & 0.38 \\
\hline
\end{tabular}

Tabela 53. Previsão um passo a frente para a série gerada

\begin{tabular}{l|c|c}
\hline \multicolumn{1}{c|}{ Meses } & Valor Real & Valor Previsto \\
\hline Janeiro & 0.7675 & 0.8832 \\
Fevereiro & -0.5297 & -0.4401 \\
Março & -0.3300 & -0.0138 \\
Abril & -0.6725 & -0.6079 \\
Maio & -0.2940 & -0.2040 \\
Junho & -0.6398 & -0.1513 \\
Julho & -0.4525 & -0.0795 \\
Agosto & 0.3229 & 0.3334 \\
Setembro & -0.9291 & -0.8191 \\
Outubro & -0.5447 & -0.5166 \\
Novembro & 0.5250 & 0.5959 \\
Dezembro & -0.2034 & 0.3871 \\
\hline EMP & 0.54 &
\end{tabular}




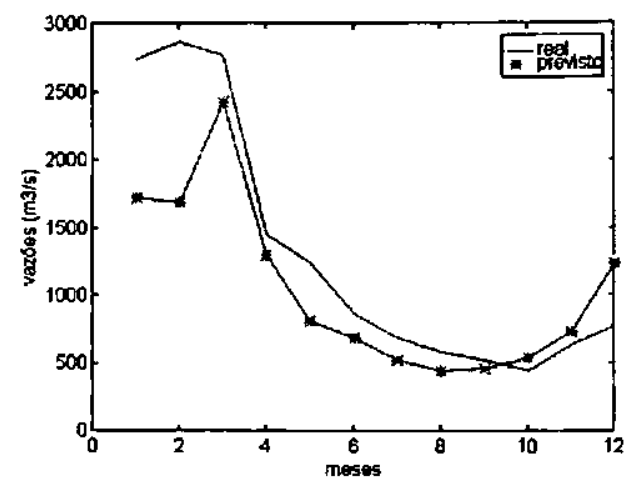

Figura 43. Previsão um passo a frente para a série de Furnas considerando a priori Normal-Gama para os meses de Janeiro a Dezembro.

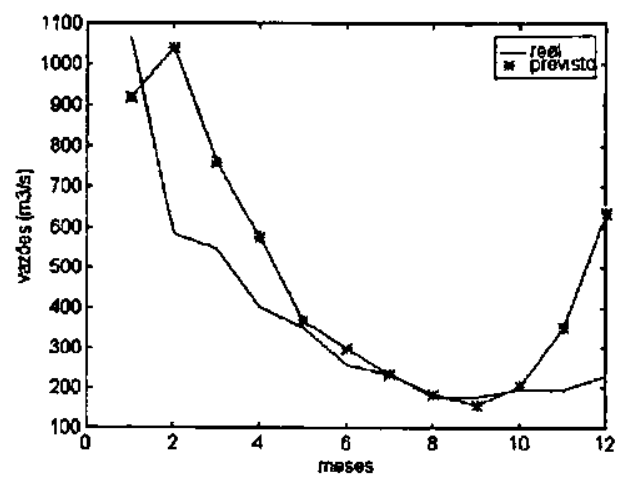

Figura 44. Previsão um passo a frente para a série de Emborcação considerando a priori Normal-Gama para os meses de Janeiro a Dezembro.

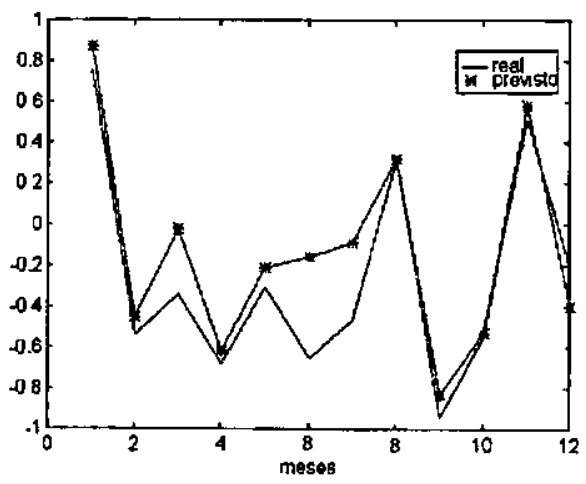

Figura 45. Previsão um passo a frente para a série gerada considerando a priori Normal-Gama para os meses de Janeiro a Dezembro. 


\section{CAPÍTULO 6}

\section{Conclusões e Perspectivas Futuras}

O uso de técnicas de simulação de Monte Carlo em Cadeias de Marvok se mostrou bastante apropriada para a análise de dados de séries temporais, quando se trabalha com séries periódicas onde envolve muitos parâmetros, e as inferências usuais baseadas na estimação de máxima verossimilhança não são viáveis. Os resultados obtidos com o estimadores de máxima verossimilhança se mostraram equivalentes aos obtidos pelo método de MCMC, além disso o uso dos algoritmos amostrador de Gibbs e Metropolis-Hastings, é de simples implementação e não exige conhecimentos computacionais sofisticados.

As densidades preditivas são facilmente obtidas por estimadores de Monte Carlo e mostram a simplicidade e eficiência no cálculo das previsões de valores futuros. O uso do critério Bayesiano de adequabilidade do modelo baseado na densidade preditiva ordenada mostrou-se adequado e seus resultados coincidem com o critério BIC. Recomendamos usar esses critérios juntamente com os intervalos de credibilidade dos parâmetros.

As técnicas apresentadas nesta dissertação poderiam ser generalizadas a outras classes de modelos de séries temporais.

Deixamos algumas porpostas pertinentes à modelos de séries temporais, para um outro prosseguimento da pesquisa nessa área, onde destacamos: 
1. Comparar o desempenho dos modelos PAR com outros modelos normalmente usados para séries sazonais, tais como: modelos ARMA sazonal $(\mathrm{p}, \mathrm{q})(\mathrm{P}, \mathrm{Q}) \mathrm{s}$ de Box e Jenkins e modelos ARMA após a transformação da série, onde se extrai de forma determinística a sazonalidade e supõe-se a estacionariedade da componente estocática.

2. Utilizar a abordagem Bayesiana para modelos Threshold (modelos TAR) para séries periódicas de vazões, visando reduzir o número de parâmetros a serem estimados reduzindo-se o número de regimes necessários para apresentar os dados de forma adequada.

3. Comparar o desempenho dos modelos PAR e TAR para séries de vazões com dados mensais e de período de 12 meses. A medida de desempenho dos modelos pode ser feita através do erro de previsão para diferentes números de meses a frente.

4. Avaliação da robustez na inferência dos parâmetros a posteriori quando usamos densidades a priori $t$-Student e uma análise de sensibilidade na estimativas dos $\tau_{m}$ em função dos parâmetros da priori Gama. 


\section{APÊNDICE}

Neste apêndice, apresentamos os algoritmos amostrador de Gibbs, MetropolisHastings e a monitoração informal e a verificação formal da convergência de Gelman e Rubin (1992). Por fim, apresentamos uma listagem de dois programas feitos em MATLAB. versão 4.2.b.

\section{A. O algoritmo amostrador de Gibbs}

O algoritmo amostrador de Gibbs é essencialmente um algoritmo iterativo de amostragem de uma cadeia de Markov cujo núcleo de transição é formado pelas distribuições condicionais completa.

A seguir apresentamos o algoritmo amostrador de Gibbs.

Suponha que desejamos estimar a densidade a posteriori $\left\{\phi_{1, m}, \ldots, \phi_{p_{m}, m}, \tau_{m}\right\}$ condicionada aos dados, ou seja, $\Pi_{m}\left(\phi_{m}, \tau_{m} \mid \mathbf{Z}_{m}\right)$.

i-) Inicialize o contador de iterações da cadeia $j=1$ e arbitre valores iniciais:

$$
\phi_{m}^{(0)}=\left(\phi_{1, m}^{(0)}, \ldots, \phi_{p_{m}, m}^{(0)}\right)^{\prime}, \tau_{m}^{(0)}
$$

ii-) Obtenha um novo valor $\phi_{m}^{(j)}=\left(\phi_{1, m}^{(j)}, \ldots, \phi_{p_{m}}^{(j)},\right)^{\prime}, \tau_{m}^{(j)}$ a partir $\phi_{m}^{(j-1)}$ através de sucessivas gerações de valores,

$$
\phi_{m}^{(j)} \sim \Pi_{m}\left(\phi_{m} \mid \tau_{m}^{(j-1)}\right)
$$




$$
\tau_{m}^{(j)} \sim \Pi_{m}\left(\tau_{m} \mid \phi_{m}^{(j-1)}\right)
$$

iii-) Atualize o contador $j$ para $j+1$ e retorne a (ii) até a convergência.

A medida que o número de iterações aumenta, a cadeia se aproxima de sua condição de equilibrio. Assim, assume-se que a convergência é atingida em uma iteração cuja distribuição esteja arbitrariamente próxima da distribuição de equilibrio.

O algoritmo amostrador de Gibbs define uma cadeia de Markov pois os acontecimentos na iterção $j$ dependem da história do processo apenas através dos valores na iterção $j-1$.

\section{B. Algoritmo de Metropolis-Hastings}

O algoritmo de Metropolis-Hastings é utilizado quando temos interesse em gerar amostras aleatórias de distribuições condicionais não padronizadas. Supor que desejamos amostras de uma densidade não regular, definimos o núcleo de transição $q_{m}\left(\phi_{m}, \phi_{m}^{*}\right)$, da distribuição $\Pi\left(\phi_{m}\right)$, que gera $\phi_{m}^{*}$ a partir de $\phi_{m}$.

Quando a posteriori é escrita na forma $\Pi_{m}(t)=h_{m}(t) \Psi_{m}(t)$, onde $h_{m}(t)$ é uma densidade com que pode ser amostrada e $\Psi_{m}(t)$ é uma função uniformemente limitada, então $h_{m}(t)=q_{m}\left(\phi_{m}, \phi_{m}^{*}\right)$ é usado como candidato gerador.

Apresentamos o algoritmo de Metropolis-Hastings.

i-) Inicialize o contador de iterações da cadeia $j=1$ e arbitre valores iniciais:

$$
\phi_{m}^{(0)}=\left(\phi_{1, m}^{(0)}, \ldots, \phi_{p_{m}, m}^{(0)}\right)^{\prime}, \tau_{m}^{(0)}
$$


ii-) Gerar um novo valor $\phi_{m}^{*}$ a partir da função de transição $q_{m}\left(\phi_{m}, \phi_{m}^{*}\right)$.

iii-) Calcule a probabilidade de aceitação do novo valor na interação $j$ por:

$$
\alpha_{m}\left(\phi_{m}^{(j)}, \phi_{m}^{*}\right)=\left\{\begin{array}{cc}
\min \left\{1, \frac{\Psi_{m}\left(\phi_{m}^{*}\right) q_{m}\left(\phi_{m}^{(j)}, \phi_{m}^{*}\right)}{\Psi_{m}\left(\phi_{m}^{(j)}\right) h_{m}\left(\phi_{m}^{*}, \phi_{m}^{(j)}\right)}\right\} & \text { se } \Psi_{m}\left(\phi_{m}^{(j)}\right) q_{m}\left(\phi_{m}^{*}, \phi_{m}^{(j)}\right)>0 \\
1 & \text { caso contrário. }
\end{array}\right.
$$

iv-) Gerar $u$ da uniforme $U[0,1]$. Então:

$$
\phi_{m}^{(j+1)}= \begin{cases}\phi_{m}^{*} & \text { se } u \leq \alpha_{m}\left(\phi_{m}, \phi_{m}^{*}\right) \\ \phi_{m}^{(j)} & \text { caso contrário }\end{cases}
$$

\section{Monitoração Informal da convergência}

Gelfand e Smith (1990), sugeriram técnicas gráficas para verificação da convergência.

Após um número suficientemente grande $N$ de iterações e $m$ cadeias paralelas, forma-se uma amostra de $\phi_{m}$ e pode-se construir histograma de qualquer uma de suas componentes ou funções. Este histograma pode ser suavizado através de alguma técnica de alisamento ou não. O mesmo procedimento pode ser repetido após $N+k$ iterações. Se não houver diferença perceptível a olho nu entre os gráficos obtidos após $N$ e $N+k$ iterações, então conclui-se pela convergência das cadeias. $\mathrm{O}$ valor de $k$ não pode ser muito pequeno, pois a correlação inerente a cadeia de Markov estará exercendo sua influência e não poderá dizer que a similariedade é 
devida a convergência ou a correlação do processo. Valores de $k$ muito grande são desnecessários pois se há suspeitas de convergência após $N$ iterações não há necessidade de ir muito além da cadeia apenas para uma verificação.

As técnicas de monitoração gráfica devem ser usadas com cautela, pois podem ser ilusórias indicando uma constância que pode não ser tão evidente sob outra escala. Além disso, muitas cadeias podem exibir comportamento similar ao de convergência sem que a convergência tenha sido atingida.

\section{Verificação Formal de Convergência}

Uma técnica simples de verificar convergência é proposto por por Gelman e Rubin (1992), o qual consiste em considerarmos várias cadeias em paralelo começando de diferentes pontos. Com isso, evita-se que as cadeias se concentrem em regiões em torno de uma moda local, no caso de multimodalidade da posteriori. Após convergência, todas as cadeias devem ter o mesmo comportamento qualitativo e quantitativo mas a verificação de convergência pode ser problemática se for analisada apenas uma cadeia.

A verificação visual de similaridade entre as trajetórias das cadeias após algumas iterações certamente é um indício forte de convergência. Gelman e Rubin (1992) elaboraram sobre essa idéia e propuseram alguns métodos formais para detecção de convergência além dessa detecção visual.

Considerando $m$ cadeias paralelas e uma função real $t(\theta)$, tem-se $m$ trajetórias $\left\{t_{i,}^{(1)} t_{i, \ldots}^{(2)} t_{i,}^{(n)}\right\}, i=1, \ldots, m$ para $t$. Portanto, podem ser obtidas a variância entre as 
cadeias $E$ e a variância dentro das cadeias $D$ dadas por:

$$
\begin{gathered}
E=\frac{n}{m-1} \sum_{i=1}^{m}\left(\bar{t}_{i}-\bar{t}\right)^{2} \\
D=\frac{1}{m(n-1)} \sum_{i=1}^{m} \sum_{j=1}^{n}\left(t_{i}^{(j)}-\bar{t}_{i}\right)^{2}
\end{gathered}
$$

onde $\bar{t}_{i}$ é a média das observações da cadeia $i$ e $\bar{t}$ é a média dessas médias, $i=1, \ldots, m$. Sob convergência, todos os $m n$ valores serão gerados da posteriori e a variância de $t$ pode ser estimada de forma não viciada por:

$$
\widehat{\operatorname{Var}}[t(\theta)]=\left(1-\frac{1}{n}\right) D+\left(\frac{1}{n}\right) E
$$

Se as cadeias ainda não tiverem convergido então essa estimativa é maior que $\widehat{\operatorname{Var}}[t(\theta)]$ pois os valores iniciais ainda estarão influenciando e eles foram escolhidos com dispersão maior que a da distribuição de equilibrio. Por outro lado, $D$ fornece estimativas menores que $\widehat{\operatorname{Var}}[t(\theta)]$ pois uma cadeia só não terá coberto toda a variabilidade de $t(\theta)$. Um indicador de convergência é dado pela redução potencial estimada da escala $R=\sqrt{\widehat{\operatorname{Var}}[t(\theta)] / D}$ que é sempre maior que 1 .

A medida que $n$, cresce ambos os estimadores acabarão convergindo para $\widehat{\operatorname{Var}}[t(\theta)]$ e $R$ convergirá para 1 . Logo $R$ pode ser usado como indicador de convergência pela avaliação de sua proximidade a 1. Gelman (1995) sugere aceitar convergência quando o valor de $R$ calculado para todas as funções $t$ de interesse for da ordem 1.1. 


\section{E. Programas}

Utilizando o sistema computacional MATLAB, apresentamos dois dos programas usados nesta dissertação. Os programas são utilizados para encontrar as densidades a posteriori marginais para $\phi_{m}$ e $\tau_{m}$ e o critério de convergência de Gelman e Rubin.

PROGRAMA DO ALGORITMO AMOSTRADOR DE GIBBS PARA A PRIORI CONJUGADA NORMAL-GAMA.

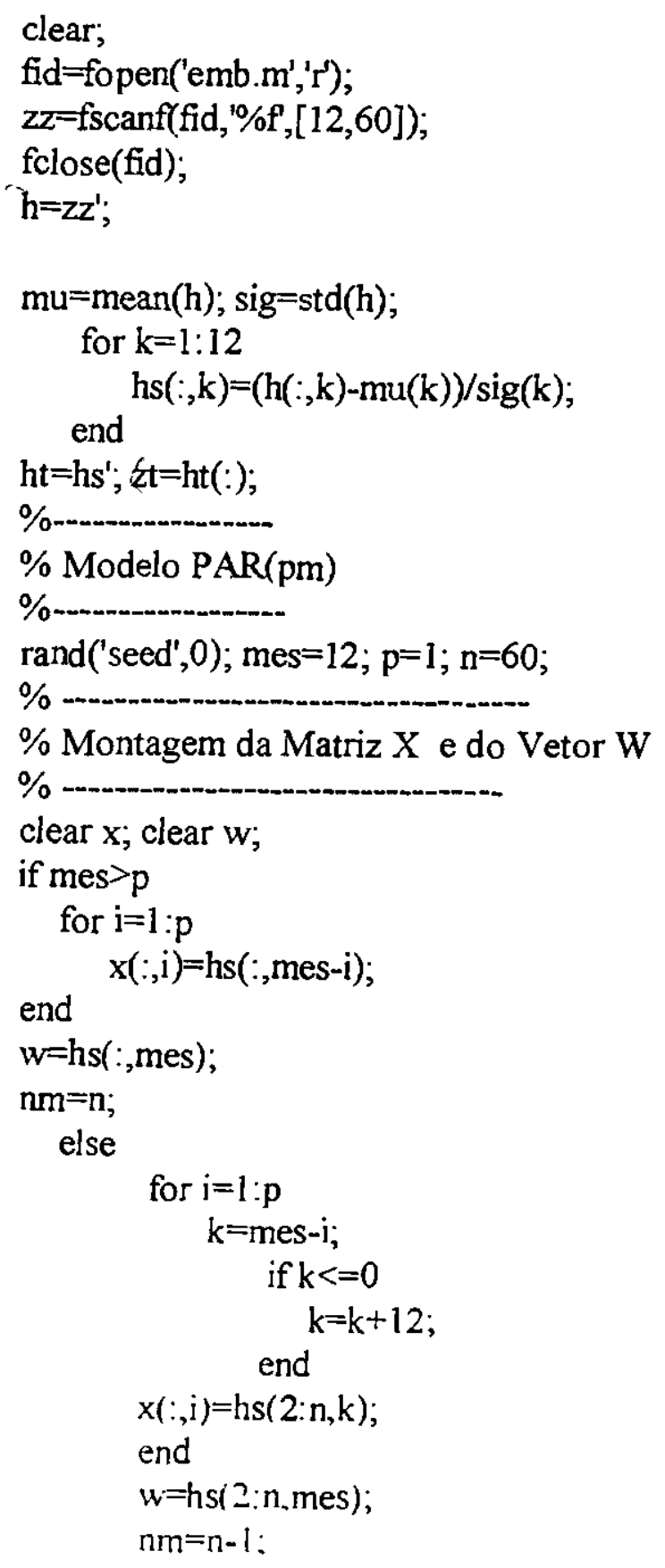


phic $=\operatorname{inv}\left(x^{\prime *} x\right)^{*}\left(x^{\prime *} w\right)$;

$\% \mathrm{EMV}$

tauc $=n m /\left(\left(w-x^{*} p h i c\right) * *\left(w-x^{*}\right.\right.$ phic $\left.)\right) \%$ EMV

$\%$

$\%$ desvio-padrao

$\%$-.............

sdphic=sqrt $\left(\operatorname{diag}\left((1 / \operatorname{tauc}) * \operatorname{inv}\left(\mathrm{x}^{\prime *} \mathrm{x}\right)\right)\right) ; \operatorname{sdtauc}=\operatorname{sqrt}\left((2 / \mathrm{nm})^{*}\left(\operatorname{tauc}{ }^{\wedge} 2\right)\right)$;

disp(mes)

disp(['série de Emborcação - E.M.V. '])

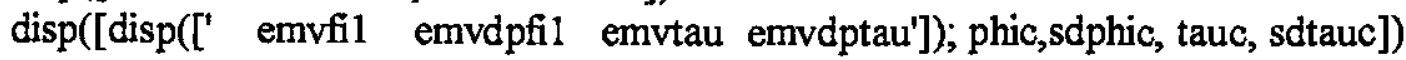

$\%$

$\%$ prioris para tau - Gama (alfa,beta)

$\%$

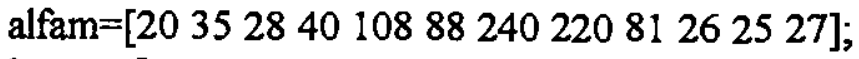

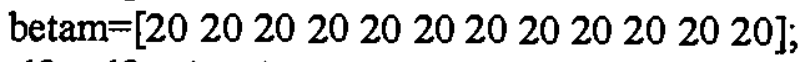

alfa $=$ alfam(mes);

beta=betam(mes);

$\%$

$\%$ prioris para phi - Normal (mu_phi,tau*prec)

$\%$

prec $=30^{*}$ eye $(p, p) ; \quad \%$ matriz precisao

mu_phi=phic; $\quad \%$ media

$\%$

$\%$

$\%$ condicoes iniciais

$\%$

for $k=1: p$

phi $(k, 1)=0.8$;

phi_k $1, \mathrm{k})=$ phi $(\mathrm{k})$;

end

$\operatorname{tau}(1)=1$;

$\%$

$\%$ Inicio do Programa Principal

$\%$

nint $=4000$;

no $=2000$;

cont $=0$;

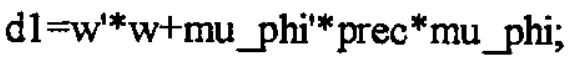

$\mathrm{d} 2=\left(\mathrm{x}^{1 *} \mathrm{w}+\text { prec }{ }^{*} \mathrm{mu} \_ \text {phi }\right)^{1 *} \operatorname{inv}\left(\mathrm{x}^{\prime *} \mathrm{x}+\text { prec }\right)^{*}\left(\mathrm{x}^{1 *} \mathrm{w}+\right.$ prec ${ }^{*} \mathrm{mu} \_$phi $)$;

$\mathrm{d}=\mathrm{d} 1-\mathrm{d} 2$;

phitil $=\operatorname{inv}\left(x^{\prime *} x+\right.$ prec $) *\left(x^{\prime *} w+\right.$ prec*mu_phi $)$; 


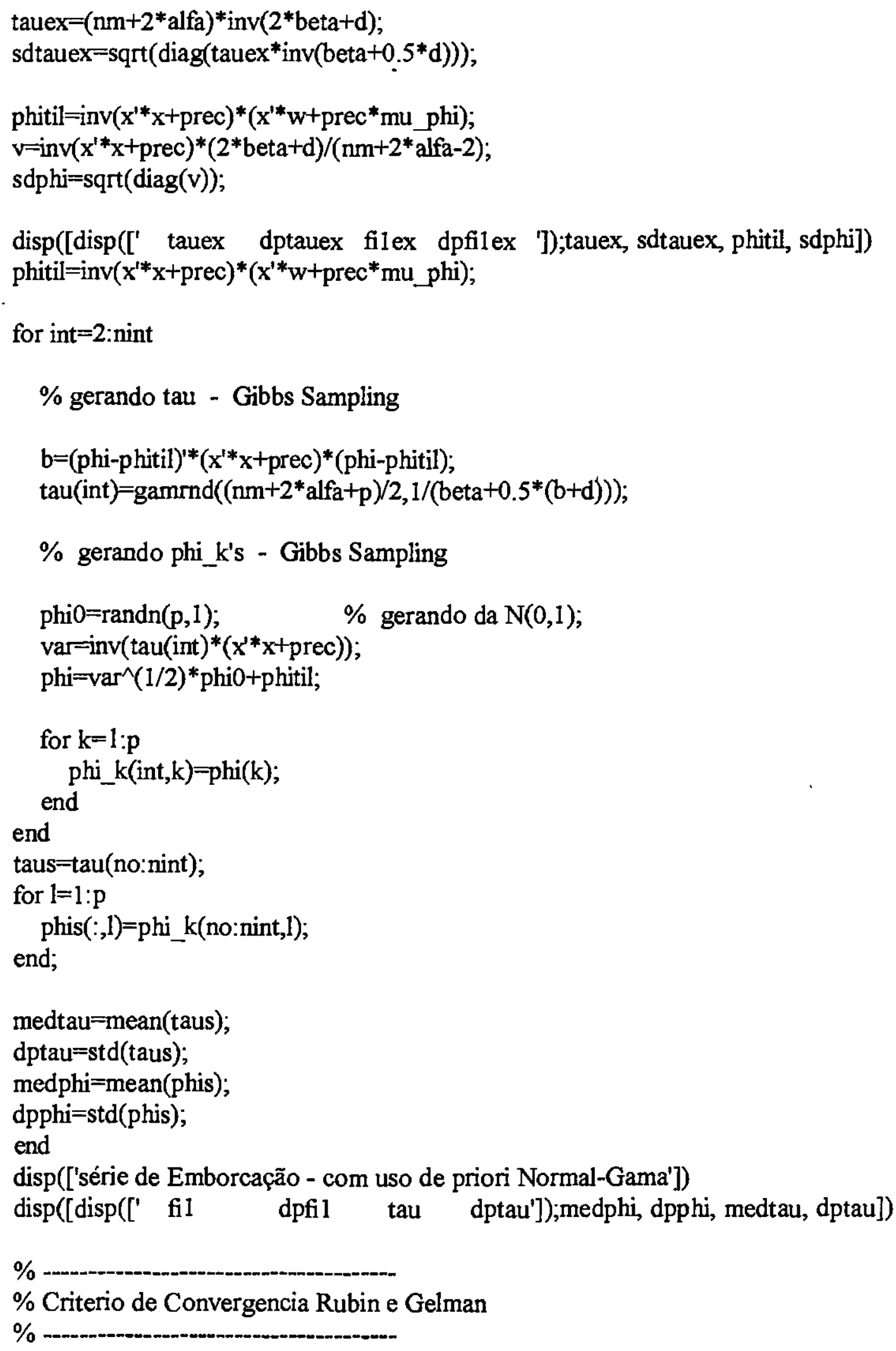


fil $x=$ phis(:, 1); tau $1 x=$ taus; $n c=2 ; n 1=1 ; n 2=$ length(fil $x) / n c ; d=n 2-n 1+1$;

Mefil $=[\operatorname{mean}($ fil $1 \times(n 1: n 2))$, mean(fil $x(n 1+d: n 2+d))]$;

QMfil=Mefil.*Mefil;

Vafil $=[\operatorname{cov}(f i 1 x(n 1: n 2)), \operatorname{cov}(f i 1 x(n 1+d: n 2+d))]$;

MeMfil =sum(Mefi1)/nc;

Wfil=sum $($ Vafil $) / \mathrm{nc}$;

VarVfil $=\left(\right.$ Vafil ${ }^{*}$ Vafil'-nc*Wfil`2)/(nc-1);

Ufil =d*(Mefi1 *Mefil'-nc*MeMfi 1^2)/(nc-1);

VARfi $1=((\mathrm{d}-1) / \mathrm{d}) * \mathrm{Wfil}+(1 / \mathrm{d}) *$ Ufil;

VHfil=VARfil+Ufil/(nc*d);

Mcovfil $=\operatorname{cov}($ Vafil, Mefi1 $)$;

Qcovfi1=cov(Vafil,QMfi1);

COVfil $=$ Qcovfi 1(1,2)-2*MeMfil *Mcovfi 1(1,2);

$\operatorname{VarVHfil}=((\mathrm{d}-1) / \mathrm{d})^{\wedge} 2^{*}(1 / \mathrm{nc})^{*} \operatorname{VarVfi} 1+\left(((\mathrm{nc}+1) /(\mathrm{nc} * \mathrm{~d}))^{\wedge} 2\right)^{*}(2 /(\mathrm{nc}-$

1))*Ufi $1^{\wedge} 2+2 * \operatorname{COVfil}^{*}(\mathrm{nc}+1) *(\mathrm{~d}-1) /\left(\mathrm{d}^{*} n \mathrm{c}^{\wedge} 2\right)$;

dffil $=2 *($ VHfil $1 \wedge$ )/VarVHfil;

Rfil=sqrt((VHfi 1/Wfil)*dffi $1 /(\operatorname{dffi} 1-2))$;

Metaul $=[\operatorname{mean}(\operatorname{tau} 1 x(n 1: n 2))$, mean $(\operatorname{tau} 1 x(n 1+d: n 2+d))]$;

QMtau1=Metau1.*Metau1;

Vatau $1=[\operatorname{cov}(\operatorname{taulx}(\mathrm{n} 1: \mathrm{n} 2)), \operatorname{cov}(\operatorname{tau} 1 x(\mathrm{n} 1+\mathrm{d}: \mathrm{n} 2+\mathrm{d}))]$;

MeMtaul=sum(Metau1)/nc;

Wtaul=sum(Vatau 1$) /$ nc;

VarVtau1=(Vatau1*Vataul'-nc*Wtau1^2)/(nc-1);

Utaul=d*(Metaul*Metaul'-nc*MeMtau1^2)/(nc-1);

VARtaul $=((\mathrm{d}-1) / \mathrm{d}) * \mathrm{Wtau} 1+(1 / \mathrm{d}) *$ Utaul;

VHtaul=VARtaul+Utaul/(nc*d);

Mcovtau1=cov(Vatau1,Metau1);

Qcovtaul=cov(Vataul, QMtau 1$)$;

COVtau1=Qcovtau 1(1,2)-2*MeMtau1 *Mcovtau1 $(1,2)$;

$\operatorname{VarVHtau} 1=((\mathrm{d}-1) / \mathrm{d})^{\wedge} 2^{*}(1 / \mathrm{nc})^{*} \operatorname{Var} \operatorname{Vtau} 1+\left(\left((\mathrm{nc}+1) /\left(\mathrm{nc}^{*} \mathrm{~d}\right)\right)^{\wedge} 2\right)^{*}(2 /(\mathrm{nc}-$

1))*Utau $1^{\wedge} 2+2 * \operatorname{COVtau}^{*}(\mathrm{nc}+1) *(\mathrm{~d}-1) /\left(\mathrm{d}^{*} \mathrm{nc} \mathrm{c}^{\wedge} 2\right)$;

dftau $1=2 *($ VHtau $1 \wedge 2) /$ VarVHtaul;

Rtau1=sqrt((VHtau1/Wtau1)*dfau1/(dfau1-2));

disp(['critério de convergência Rubin e Gelman'])

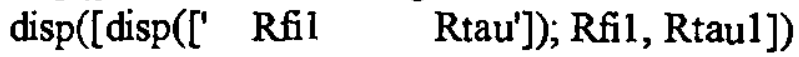

end 


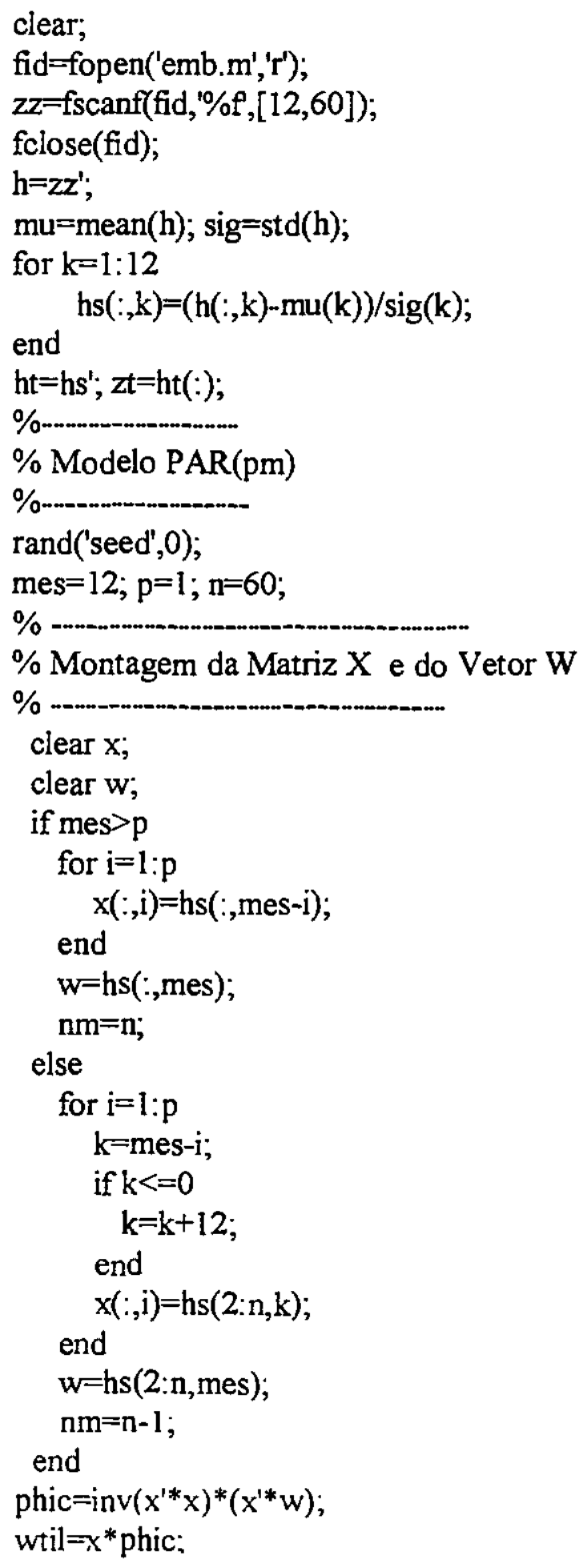




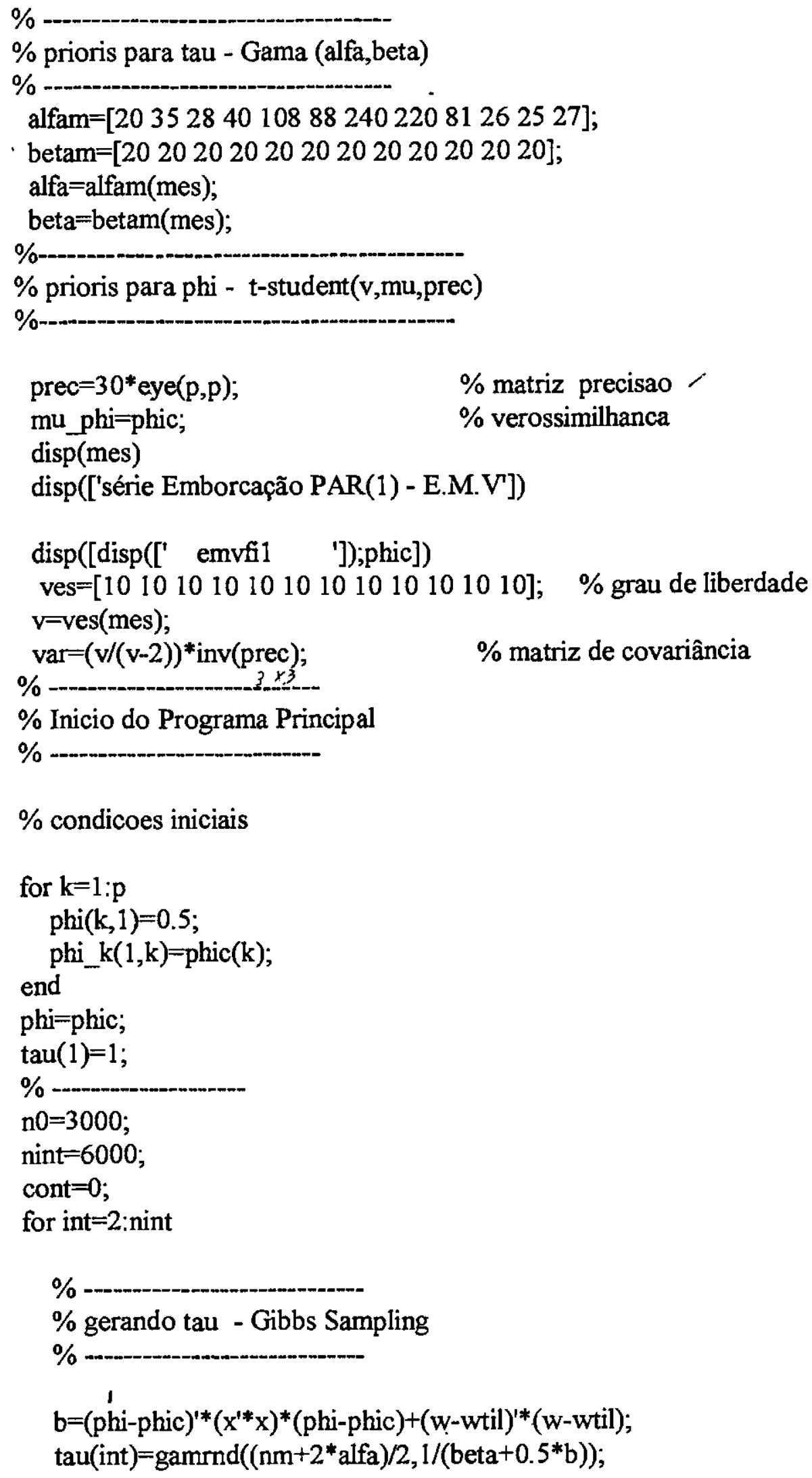

$\%$ condicoes iniciais

for $k=1: p$

$\operatorname{phi}(\mathrm{k}, 1)=0.5$;

phi_k $(1, \mathrm{k})=$ phic $(\mathrm{k})$;

end

phi=phic;

$\operatorname{tau}(1)=1$;

$\%$

$\mathrm{n} 0=3000$;

nint $=6000$;

cont $=0$;

for int $=2$; nint 
\% gerando phil e phi2 - Metropolis-Hastings

psi_old $=\exp \left((-\operatorname{tau}(\text { int }) / 2)^{*}(\text { phi-phic })^{\prime *}\left(\mathrm{x}^{\prime *} \mathrm{x}\right)^{*}(\right.$ phi-phic $\left.)\right)$;

$\%$ gerando novo candidato - usando a priori como núcleo

phi $0=$ trnd $(\mathrm{v}, \mathrm{p}, 1) ; \quad \%$ gerando da $t$-Student

phinew $=\operatorname{var}^{\wedge}(1 / 2)^{*}$ phi0+mu_phi;

for $k=1: p$ $9 x=3$

phink $($ int $-1, k)=$ phinew $(k)$;

end

psi_new $=\exp \left((-\operatorname{tau}(\mathrm{int}) / 2)^{*}(\text { phinew-phic })^{\prime *}\left(\mathrm{x}^{* *} \mathrm{x}\right)^{*}(\right.$ phinew-phic $\left.)\right)$;

if psi_old $\sim=0$

prob $=\min \left(1, p s i \_n e w / p s i \_o l d\right)$;

else

prob $=1$;

end

$\mathrm{u}=\mathrm{rand}$;

if $\mathbf{u}<=$ prob

for $\mathrm{k}=1: \mathrm{p}$

phi(k)=phinew $(k)$;

end

cont $=$ cont +1 ;

end

for $k=1: p$

phi_k(int,k)=phi(k);

end

end

phis=phi_k(1+n0:15:nint,:);

taus $=\operatorname{tau}(1+\mathrm{n0}: 15$ :nint $)$;

$\%$

$\%$ Cálculo dos intervalos de Credibilidade

$\%$

tord=sort(taus);

$11=$ tord $(0.025 *$ length(taus) $) ; 12=\operatorname{tord}(0.975 *$ length(taus $))$;

pl ord=sort(phis(:,1)); 13=plord(0.025*length(phis(:,1))); 14=plord(0.975*length(phis(:,1)));

medtau=mean(taus);

dptau=std(taus);

medphi=mean(phis);

dpphi=std(phis);

$\%$ 
$\%$ Criterio de Convergencia Rubin e Gelman

$\%$

fil $x=$ phis $(:, 1) ;$ taul $x-$ taus; $n c=2 ; n 1=1 ; n 2=$ length(filx)/nc; $d=n 2-n 1+1$;

Mefil $=[\operatorname{mean}(f i 1 x(n 1: n 2)), \operatorname{mean}(f i 1 x(n 1+d: n 2+d))]$;

QMfil=Mefil.*Mefil;

Vafil $=[\operatorname{cov}($ fil $x(n 1: n 2)), \operatorname{cov}(f i 1 x(n 1+d: n 2+d))]$;

MeMfil=sum(Mefil)/nc;

Wfil=sum(Vafi 1$) /$ nc;

VarVfil =(Vafil $*$ Vafil'-nc*Wfil^2)/(nc-1);

Ufil =d*(Mefi1*Mefil'-nc*MeMfi1^2)/(nc-1);

VARfi $1=((\mathrm{d}-1) / \mathrm{d}) * \mathrm{Wfi} 1+(1 / \mathrm{d}) * \mathrm{Ufi} 1$;

VHfil=VARfil+Ufil/(nc*d);

Mcovfil $=\operatorname{cov}($ Vafil,Mefi1);

Qcovfil=cov(Vafil,QMfi1);

Covfil $=$ Qcovfi1 $(1,2)-2 *$ MeMfil *Mcovfi $1(1,2)$;

VarVHfil $=((d-1) / d)^{\wedge} 2 *(1 / n c) * \operatorname{VarVfil} 1+\left(\left((n c+1) /\left(n c^{*} d\right)\right)^{\wedge} 2\right)^{*}(2 /(n c-$

1))*Ufi ${ }^{\wedge} 2+2 * \operatorname{COVfil}^{*}(\mathrm{nc}+1) *(\mathrm{~d}-1) /\left(\mathrm{d}^{*} \mathrm{nc}^{\wedge} 2\right)$;

dffi $1=2 *($ VHfi $1 \wedge 2) /$ VarVHfil;

Rfil=sqrt ((VHfi $1 /$ Wfil $\left.)^{*} \mathrm{dffil} /(\operatorname{dffi} 1-2)\right)$;

Metaul $=[\operatorname{mean}(\operatorname{tau} 1 x(n 1: n 2))$, mean $(\operatorname{tau} 1 x(n 1+d: n 2+d))]$;

QMtau 1=Metau1.*Metau1;

Vatau $1=[\operatorname{cov}(\operatorname{tau} 1 x(n 1: n 2)), \operatorname{cov}(\tan 1 x(n 1+d: n 2+d))]$;

MeMtaul=sum(Metau 1$) /$ nc;

Wtaul=sum(Vataul)/nc;

VarVtaul $=\left(\right.$ Vatau $1^{*}$ Vataul'-nc*Wtau $\left.1^{\wedge} 2\right) /($ nc-1);

Utaul $1=d^{*}\left(\right.$ Metaul ${ }^{*}$ Metaul'-nc*MeMtau $\left.1^{\wedge} 2\right) /($ nc-1);

VARtaul $=((\mathrm{d}-1) / \mathrm{d}) * \mathrm{Wtau} 1+(1 / \mathrm{d}) * \mathrm{Utau} 1$;

VHtaul=VARtaul+Utaul/(nc*d);

Mcovtaul=cov(Vataul,Metaul);

Qcovtau1=cov(Vatau1,QMtau1);

COVtau 1=Qcovtau 1(1,2)-2*MeMtau 1*Mcovtau 1 $(1,2)$;

$\operatorname{VarVHtaul}=((\mathrm{d}-1) / \mathrm{d})^{\wedge} 2^{*}(1 / \mathrm{nc})^{*} \operatorname{VarVtau} 1+\left(((\mathrm{nc}+1) /(\mathrm{nc} * \mathrm{~d}))^{\wedge} 2\right)^{*}(2 /(\mathrm{nc}-$

1))*Utaul^2+2*COVtau $1^{*}(\mathrm{nc}+1)^{*}(\mathrm{~d}-1) /\left(\mathrm{d}^{*} \mathrm{nc} \mathrm{c}^{\wedge} 2\right)$;

dftau $1=2 *($ VHtau $1 \wedge 2) /$ VarVHtaul;

Rtau 1=sqrt((VHtau 1/Wtaul)*dftaul/(dftaul-2)); 


\section{REFERÊNCIAS}

Adams, G. J.; Goodwin,G.C. (1995). Parameter estimation of Periodic ARMA Models. Journal of Time Series Analysis, vol. 16, no. 2, 127-145.

Anderson, P.L.; Vecchia, A. V. (1993). Asymptotic Result for Periodic Autoregressive-Moving Average Processes. Journal of Time Series Analysis Anal., 1,1-8.

Andrade, M.G.; Carvalho, M. F. H.; Barone, J. C. (1987). Modelos para Previsão do Consumo de Energia Elétrica.

Bartlett, M. S. (1946). On the Theorical Specification and Sampling Properties of Autocorrelated Time Series. J. R. Statist. Soc., vol 8, 27-41.

Bohnakov, G. N. (1996). Recursive Computation of the Parameters of Periodic Autoregressive Moving-Average Processes. Journal of Time Series Analysis, vol. 17 , no. 4 .

Box, G.E. P.; Jenkins, G. M.; Reisel,G. C. (1994). Time Series Analysis: Forecasting and Control. Holden-Day.

Box, G.E. P.; Tiao, G. C. (1973),-Bayesian Inference in Statistical Analysis. New York, Addison. Wesley.

Broemeling, L. D.; Cook, P. (1992)- Bayesian Analysis of Threshold Autoregressions. Commun. Statist, 21, 9, 2459-2482.

Broemeling, L. D. (1985). Bayesian Analysis of Linear Models. Marcel Dekker.

Broemeling, L. D.; Land, M. (1984). On Forecasting with Univariate Autoregressive Process: A Bayesian Approach. Commun Statist. Theor. Meth., 13(11), 1305-1320.

Casella, G.; George, E. J. (1992). Explaining the Gibbs Sampler. The American Statistician, 46,3,167-174.

Chib, S.;Greenberg, E. (1995). Understanding the Metropolis-Hastings Algorithm. The American Statistician, 49,4,327-335.

Gelman, A. E.; Rubin, D. (1992). Inference from Iterative Simulation using Multiple Sequences. Statistical Science, 7, 457-472. 
Gelfand, A. E.; Smith, A. F. M. (1990a). Sampling-Based Approach to Cälculating Marginal Densities. Journal of the Statistical Association, 85, 410", 398-409.

Gelfand, A. E.; Smith, A. F. M. (1990b). Ilustration of Bayesian Inference in Normal Data Models Using Gibbs Sampling. Journal of the Statistical Association, $85,412,972-985$.

Jones, R. H.; Breslford, W. M. (1967). Time Series with Periodic Structure. Biometrika, 54, 403-407.

McLeod, A. L. (1994). Diagnostic Checking of Autoregressive Models with Application. Journal of Time Series-Analysis, vol. 15, no. 2, 221-233.

Tiao, G. C.; Grupe, M. R. (1980). Hidden Periodic Autoregressive Moving Average Models in Time Series Data. Biometrika, vol 67, no.2, 365-373.

Vecchia, A. V.; Ballerini, R. (1991). Testing for Periodic Autocorrelations in Seasonal Time Series Data. Biometrikä, vol 78, no.1, 53-63.

Vecchia, A. V.(1985). Maximum Likelihood Estimation for Periodic Autoregressive-Moving Average Models. Technometrics, 27, 375-384. 